Measurement of

Hydraulic Diffusivity of

Wedge-Shaped Aquifers

Drained by Streams

GEOLOGIGAL SURVEY PROFESSIONAL PAPER 514 



\section{Measurement of}

Hydraulic Diffusivity of

Wedge-Shaped Aquifers

Drained by Streams

By R. W. STALLMAN and I. S. PAPADOPULOS

GEOLOGICAL SURVEY PROFESIONAL PAPER 514

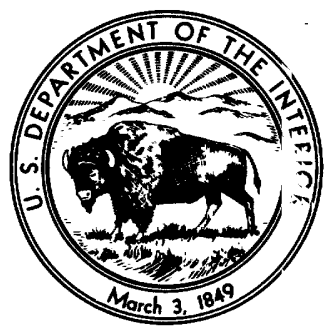

UNITED STATES GOVERNMENT PRINTING OFFICE，WASHINGTON : 1966 


\section{UNITED STATES DEPARTMENT OF THE INTERIOR \\ STEWART L. UDALL, Secretary \\ GEOLOGIGAL SURVEY \\ William T. Pecora, Director}

Library of Congress catalog-card $\cdot$ No. GS66-178 


\section{CONTENTS}

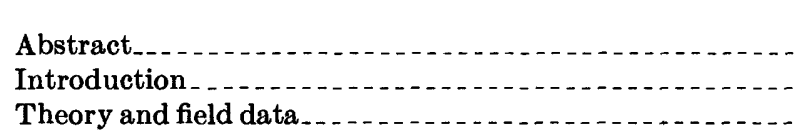

Use of response curves

1 References. 1 Tables_.

Page

\section{ILLUSTRATIONS}

PLates 1-120. Graphs showing $s / s_{0}$ versus $T t / r^{2} S$ for selected values of $\theta_{0}, \theta / \theta_{0}, r / a \ldots$

FIGURE 1. Sketch map of surface drainage pattern, showing locations of observation wells

2. Example hydrograph from well $A$ of figure 1

3. Graph of $s / s_{0}$ versus time, showing computation of $T / S_{-}$ 



\title{
MEASUREMENT OF HYDRAULIG DIFFUSIVITY OF WEDGE-SHAPED AQUIFERS DRAINED BY STREAMS
}

\author{
By R. W. Stalluman and I. S. Papadopulos
}

\begin{abstract}
Theoretical response curves are presented in graphical and tabular form relating (1) water-level changes caused by dissipation of recharge from unconfined wedge-shaped aquifers to streams to (2) $T t / r^{2} S$, from which $T / S$ can be computed.
\end{abstract}

\section{INTRODUCTION}

Many of the unconfined aquifers in the United States are drained by perennial streams. Such aquifers generally receive recharge from precipitation, part of which flows laterally to streams and part of which may be lost directly from the zone of saturation by evapotranspiration. Aquifers hydraulically bounded by streams, recharged by relatively infrequent releases of water through the unsaturated zone, and isolated from the vagaries of evapotranspiration by a thick unsaturated zone are ideally susceptible to analysis for hydraulic diffusivity-the ratio $T / S$, where $T$ is the transmissibility and $S$ is the coefficient of storage of the aquifer.

Study of the effects of large-scale development of ground water requires estimates of values of $T$ on a large scale. Pumping tests, using wells or well fields to provide a known stimulus to ground-water movement, are most generally used for determining $T$ and $S$ of aquifers. (See Ferris and others, 1962, for example.) However, pumping wells can sample only a small segment of the aquifer, and inconsistencies in $T$ and $S$ as observed from pumping tests may therefore reflect local heterogeneity and may not provide reliable values of either coefficient on an areal basis. The senior author had the problem of determining values of $T$ and $S$ on a large scale in connection with fieldwork done in Portage County, Wis. (Weeks and others, 1964).

In the vicinity of the Little Plover River, Portage County, Wis., an unconfined aquifer of outwash extends to a depth of about 80 feet below the land surface. In most years the principal source of recharge is the water released from the unsaturated zone by the spring thaw, except during summers having aboveaverage precipitation. Loss of water from the aquifer by evapotranspiration seems to be negligible. The aquifer is drained by streams in the area.
The physical system identified here is an alogous to the conditions of a heat-flow problem solved by Jaeger (1942). Jaeger's solution (1942) was applied specifically to heat flow in a split log, or the sector of a circle, where the temperature is changed on the surface of the $\log$ or perimeter of the sector of a circle at time $t=0$. Flow of heat along the longitudinal axis of the $\log$, or perpendicular to the surface of tha circle, is assumed to be negligible. Jaeger's analysis of the problem resulted in an integral equation. Numerical values of Jaeger's integral equation were ohtained by Papadopulos (1963) for use in analyzing data from three observation wells in Wisconsin (Weeks, 1964).

The close fit between observed and theoretical water-level changes encouraged development of a nearly complete set of response curves from Jaeger's work. Numerical evaluation of Jaeger's integral equation required excessively lengthy manual computation; consequently, the computing work was assigned to the U.S. Geological Survey's Computation Unit, Washington, D.C. All the response curves prosented in this paper were developed on digital equipment by that unit, under the direction of Walter Anderson.

Ground-water levels are observed in a continuing program by the Ground Water Branch, U.S. Geological Survey, in several thousand wells throughout the United States. Data from wells in unconfined aquifers, where the hydrologic controls on the aquifer are as outlined above, can be analyzed for $T / S$ by means of the response curves presented in this report.

\section{THEORY AND FIELD DATA}

A hypothetical distribution of streams is shown on figure 1. Each stream segment drains part of an unconfined aquifer underlying the area. C'sservation well A (bottom half of fig. 1) is near the confluence of two tributaries. The tributaries form a we tge having an angle of $\theta_{0}$ which equals $75^{\circ}$, approximetely. The wedge shape and location are drawn as an approximation of the course of the meandering stre $9 m s$. The angle between one side of the wedge, and a line through the apex of the wedge and observation well is noted as $\theta$ and equals $15^{\circ}$. Radius, $r$, to the observation well 


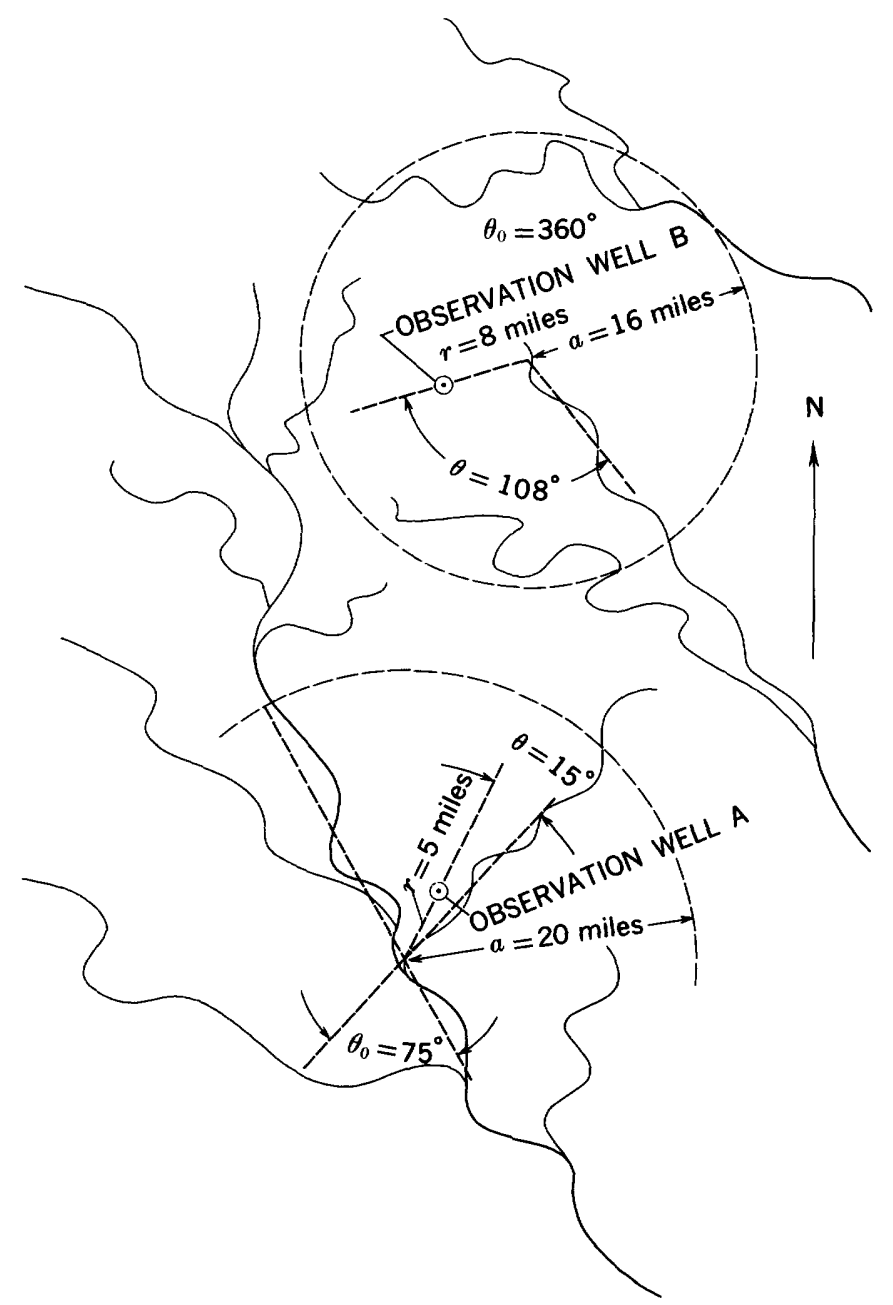

Figure 1.-Surface drainage pattern, showing location of observation wells that penetrate an unconfined aquifer.

from the apex of the wedge is approximately 5 miles. Radius $a$ is the distance from the apex of the wedge to a circumference along which water levels are presumed constant. In actuality, around well $\mathbf{A}$ the distance $a$ should be extended beyond 20 miles to the tributaries forming an approximate closure on the north. However, as will be seen, the distance $a$ can be taken as infinity without introducing a significant error in the computed $T / S$ of the area around $A$.

A hydrograph from well $\mathrm{A}$ is shown on figure 2. The decline in water level results from continued flow to the streams between January and about mid-May. During the latter part of May the aquifer is recharged by water released during the spring thaw, and the water level in well A rises sharply by an amount $s_{0}$. At the time marked $t=0$, the recharge is effectively complete and water levels begin to decline in response to discharge to the streams. The difference between the prerecharge water-level trend and observed water levels is identified as $s$ on figure 2. The time reference for $s$ is $t=0$, when recharge becomes negligible. Water-level altitudes in all streams in the area are assumed constant, as an approximation. Thus the peaks in stream stage caused by overland runoff are assumed to have negligible effect on ground-water levels.

The mathematical solution to the flow problem outlined with the aid of figures 1 and 2 is given by Jaeger (1942, p. 532, equation 16). Janger's result can be stated, for simplicity, in the following form:

$$
\frac{s}{s_{0}}=\mathbf{F}\left(\theta_{0}, \theta / \theta_{0}, r / a, T t / r^{2} S\right)
$$

where $T$ is the transmissibility of the aquifer, $S$ is the specific yield, $t$ is time measured from $t=0$ on figure 2 at which $s$ is observed, and $r$ is as defined on figure 1 . Note that $s / s_{0}, T t / r^{2} S, r / a$, and $\theta / \theta_{0}$ are nondimensional. If $t$ is in days and $r$ is in feet, the units of $T$ are square feet per day. Other notation is defined alsc on figure 1 . The function $s / s_{0}$ versus $T t / r^{2} S$ of equation 1 was evaluated for selected values of $\theta_{0}, \theta / \theta_{0}$, and $r / a$ by a digital computer.

\section{USE OF RESPONSE CURVES}

Water-level changes in the aquifer observed at well A due to lateral flow of ground water from the wedge area to the streams are given as $s / s_{0}$ verrus $\log t$ on figure 3. Figure 3 is similar to the graphs of drawdown versus the $\log$ of time commonly used for analysis of pumping-test data.

Curves of $s / s_{0}$ versus $T t / r^{2} S$ have been so compiled on plates 1-120 that each figure includes all available curves for a particular value of $\theta_{0}$ and of $\theta / \theta_{0}$. (The base sheet used for plotting was Codex No. 31,227, having 20 lines per inch, and each log cycle is $1 \frac{13 / 10}{1}$ inches wide.) Thus, to find the apprcoriate type curve for a given observation well it is necessary to first determine $\theta_{0}$ and $\theta / \theta_{0}$. From figure 1 , for well A $\theta_{0}=75^{\circ}$ and $\theta / \theta_{0}=15 / 75=0.2$. Respons 9 curves for $\theta_{0}=75^{\circ}$ and $\theta / \theta_{0}=0.2$ are on plate 34 .

The curve for $r / a=5 \mathrm{miles} / 20$ miles $=0.25$ is located on plate 34 , and is then matched with the data curve to solve for $T t / r^{2} S$ at a particular value of time. Matching is accomplished by superimposing the data plot, figure 3 , on the response curve and keeping the $s / s_{0}$ axes coincident. The data plot is moved from side to side until a position is found at which the data points overlie the theoretical response curve for $\theta_{0}=75^{\circ}$, $\theta / \theta_{0}=0.2$, and $r / a=0.25$. Any convenient value of $T t / r^{2} S$ is selected on the axes of the response curve and $t$ is read at the corresponding point on the data curve. An example match point for figure 3 is $T t / r^{2} S=1.00$ 


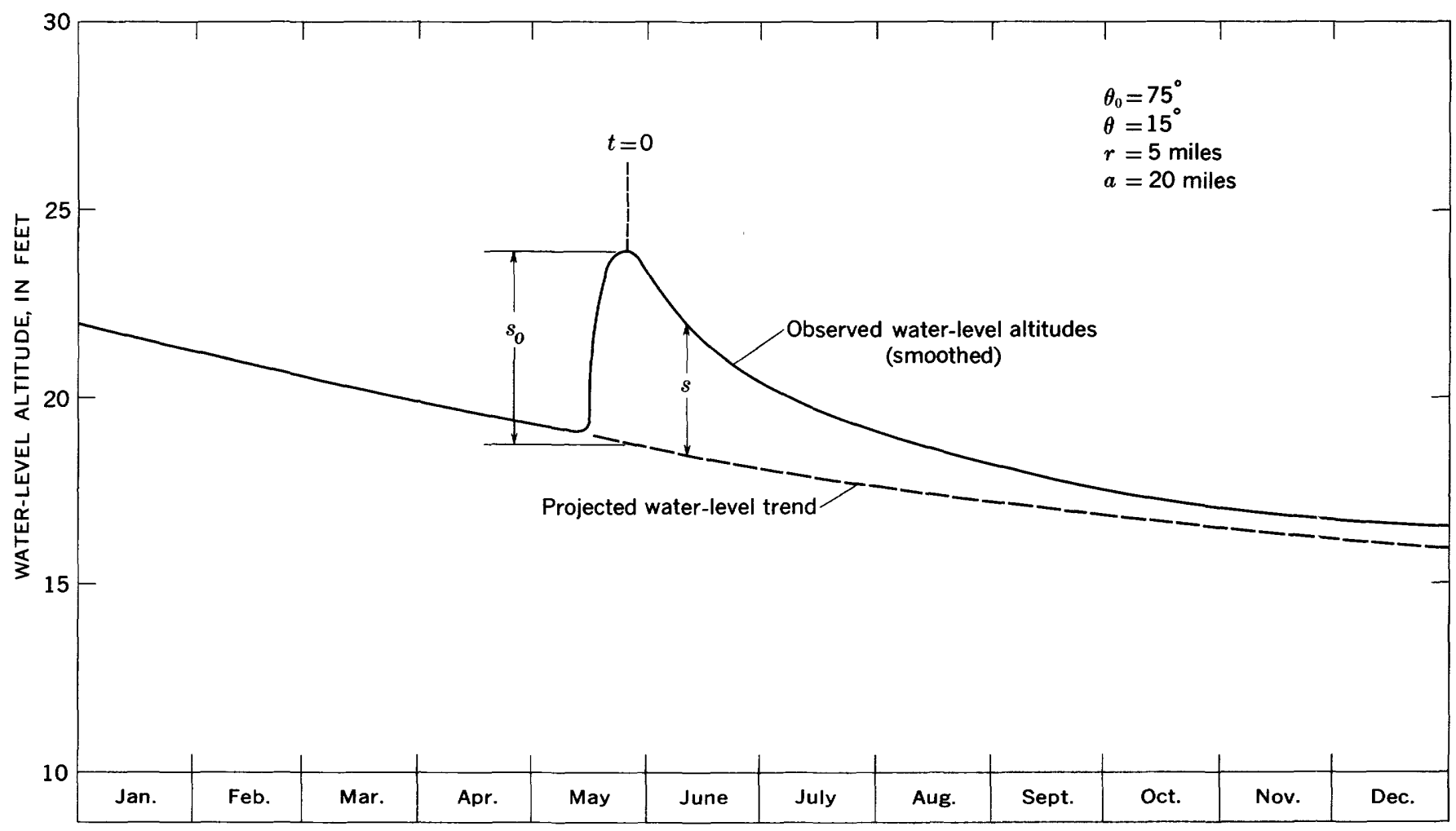

Figure 2.-Example hydrograph from well A of figure 1, showing observed and projected water-level altitudes.

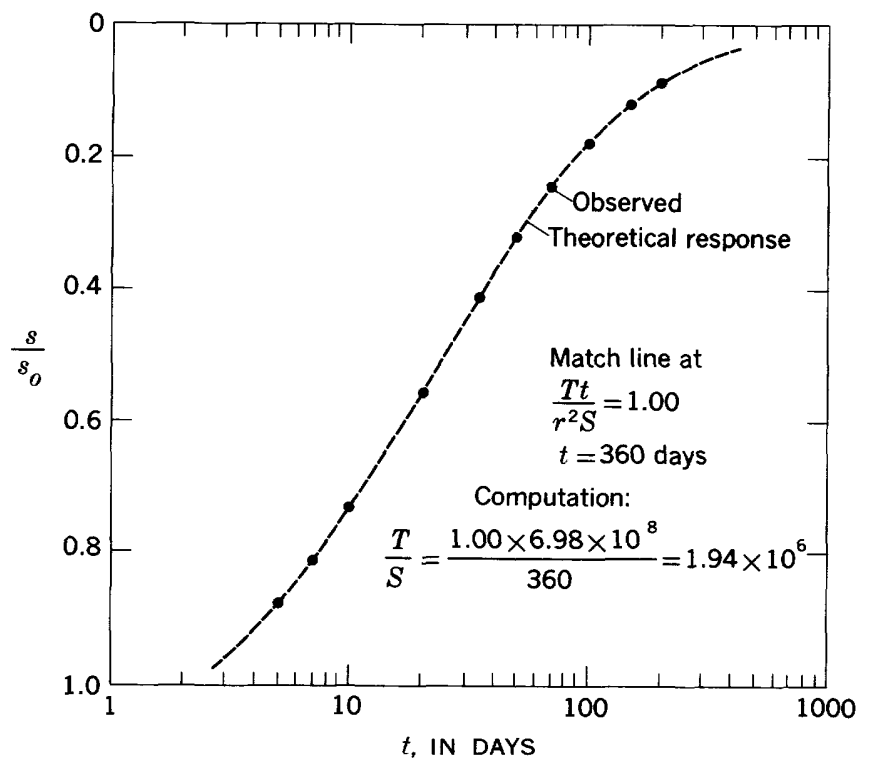

Figure $3 .-s / s_{0}$ versus time taken from hydrograph of well A (see fig. 2), showing computation of $T / S$.

and $t=360$ days. The computation for $T / S$ is given on figure 3 . If $S$ equals 0.2 ,

$T=0.2 \times 1.94 \times 10^{6}=4 \times 10^{5} \mathrm{ft}^{2}$ per day, or

$4 \times 10^{5} \times 7.48=3 \times 10^{6}$ gallons per day per foot.
As $r / a$ approaches zero, the response curves form an envelope on the lower right of many of plates 1-120. The envelope represents aquifer response for $r / a=0$, or $a=\infty$. For observation well $\mathrm{A}$, where $r / a=0.25$, response is like that where $a=\infty$ until $s / s_{0}$ is less than about 0.1 . Therefore the position assumed for radius $a$ around observation well $\mathrm{A}$ is immaterial for most of the useful data from figure 2. Increasing $a$ to a value greater than 20 miles would not affect the value of $T / S$ computed on figure 3 .

Observation well B (fig. 1) is near the headwaters of a stream. Such a situation can be viewed as a region of flow to a wedge of slightly less than $360^{\circ}$, the apex of the wedge being at the upper end of the stream. Values of the pertinent variables from figure 1 are as follows: $\theta_{0}=360^{\circ}, \theta / \theta_{0}=108 / 360=0.30$, and $r / a=0.5$. The response curve appropriate for analyzing data from $B$ is on plate 116. Note that the value of $r / a$ hos a greater effect on response for large values than for small values of $\theta_{0}$.

Caution must be used in applying the response curves. In the example hydrograph given on fig tre 2 it is difficult to project reliably the water-level trend much beyond July. Thus, values of $s / s_{0}$ from only about 50 days after cessation of recharge can be considered useful. Generally field data will be more difficult to interpret than figure 2 , because observed water leve's generally 
do not form such a smooth trend as shown. Also, consider the response of well $\mathrm{A}$ of figure 1, assuming that $T / S=2 \times 10^{4} \mathrm{ft}^{2}$ per day. Thus,

$$
\frac{T t}{r^{2} S}=\frac{2 \times 10^{4}}{6.98 \times 10^{8}} t=2.86 \times 10^{-5} t .
$$

For $t$ of about 1 year, $T t / r^{2} S$ is about $1 \times 10^{-2}$.

Therefore for $t$ less than about 1 year, $s / s_{0}$ varies between 1.0 and 0.9 if $T / S=2 \times 10^{4}, \theta_{0}=75^{\circ}, \theta / \theta_{0}=0.2$, and $r / a=0.25$. Obviously, then, stream control around well $A$ in an aquifer having $T / S$ of about $2 \times 10^{4} \mathrm{ft}^{2}$ per day could not cause the drawdowns indicated on the hydrograph of figure 2. Ground-water outflow in the area is therefore probably controlled significantly by other hydrologic factors than stream location, such as recharge conditions different than those postulated in construction of the response curves. Comparison of the response curves with observed drawdowns can be used in this manner to aid in the identification of the principal hydrologic controls on ground water.

Computed values of $1-\left(s / s_{0}\right)$ versus $T t / r^{2} S$, from which plates 1-120 were prepared, are given in the tables on pages 6-50. Two numbers are given for each row in the column marked $T t / r^{2} S$. The second is the exponent of 10 to be applied. The complete value of $T t / r^{2} S$ is obtained by multiplying the first number shown by 10 to the power indicated.

\section{REFERENCES}

Ferris, J. G., Knowles, D. B., Brown, R. H., and Stallman, R. W., 1962, Theory of aquifer tests: U.S. Geol. Survey Water-Supply Paper 1536-E, p. 69-174.

Jaeger, J. C., 1942, Heat conduction in a wedge, or an infinite cylinder whose cross-section is a circle or a sector of a circle: Philos. Mag., ser. 7, v. 33, no. 222, p. 527-536.

Papadopulos, I. S., 1963, Preparation of type curves for calculating $T / S$ of a wedge-shaped aquifer, in Srort papers in the geologic and hydrologic sciences: U.S. Geol. Survey Prof. Paper 475-B, p. B196-B198.

Weeks, E. P., 1964, Use of water-level recession curves to determine the hydraulic properties of glacial outwash in Portage County, Wisconsin, in Short papers in the geologic and hydrologic sciences: U.S. Geol. Survey Prof. Paper 501-B, p. B181-B184.

Weeks, E. P., Ericson, D. W., and Holt, C. L. R., Jr., 1965, Hydrology of the Little Plover River basin, Powtage County, Wisconsin, and the effects of water resource development: U.S. Geol. Survey Water-Supply Paper 1811, 78 p. 
TABLES OF VALUES OF NONDIMENSIONAL RESPONSE OF GROUND WATER 
HYDRAULIC DIFFUSIVITY, WEDGE-SHAPED AQUIFERS DRAINED BY STREAMS

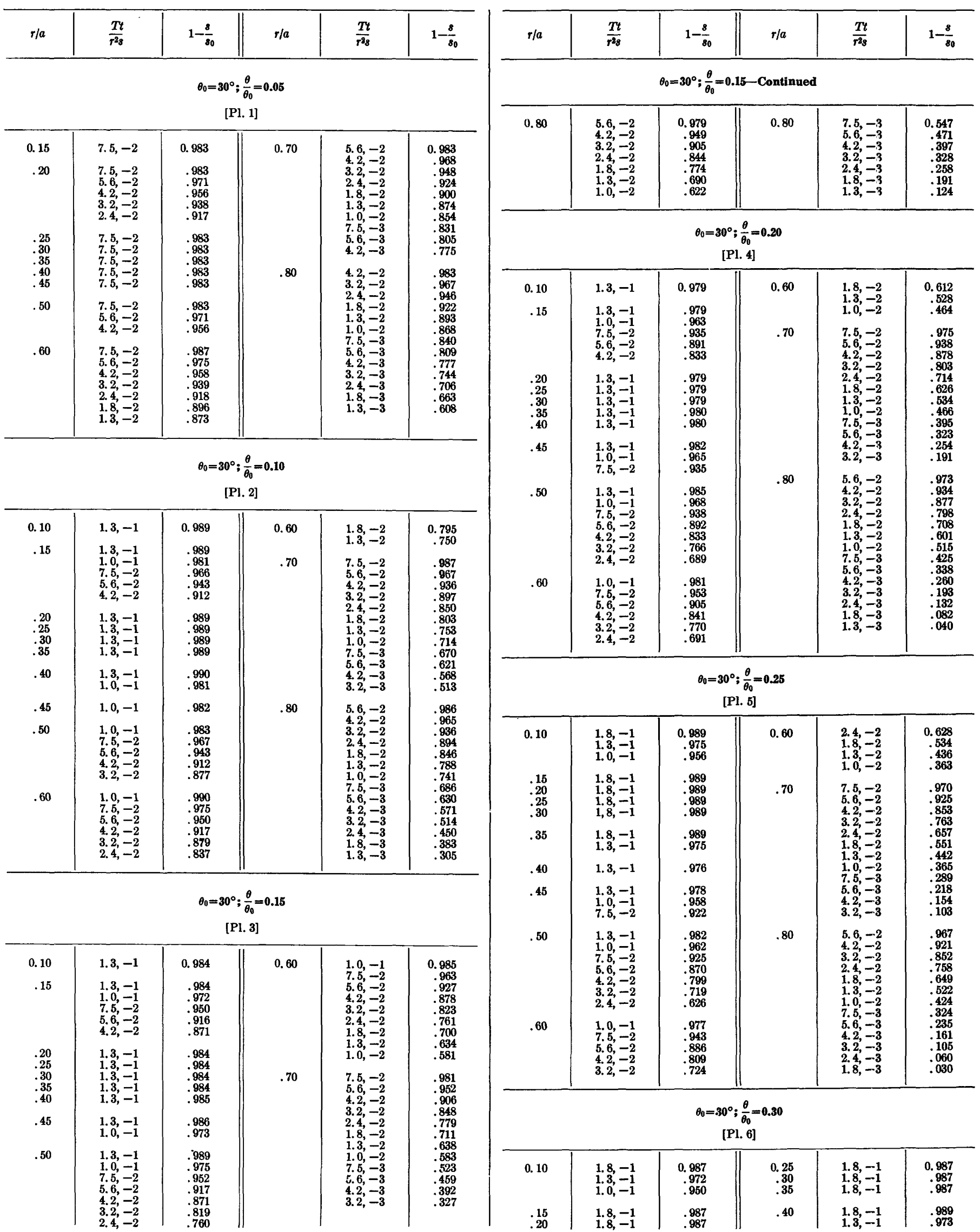




\begin{tabular}{|c|c|c|c|c|c|}
\hline$r / a$ & $\frac{T t}{r^{2} s}$ & $1-\frac{8}{80}$ & $r / a$ & $\frac{T t}{r^{2} 8}$ & $1-\frac{8}{80}$ \\
\hline \multicolumn{6}{|c|}{$\theta_{0}=30^{\circ} ;{\frac{\theta}{\theta_{0}}}^{\circ}=0.30-$ Continued } \\
\hline 0.45 & $\begin{array}{l}1.3,-1 \\
\text { 1. } 0,=1 \\
\text { 5.5, } 62\end{array}$ & $\begin{array}{r}0.975 \\
.952 \\
.911 \\
.850\end{array}$ & 0.70 & $\begin{array}{l}5.6,-2 \\
4.2,-2 \\
3.2,-2 \\
2.4,-2\end{array}$ & $\begin{array}{r}0.915 \\
.832 \\
.729 \\
.607\end{array}$ \\
\hline .50 & $\begin{array}{l}\text { 1. } 3,-1 \\
\text { 1.0, } \\
\text {. } 5,-2 \\
\text { 5. } 6,-2 \\
4.2,-2 \\
3.2,-2 \\
2.4,-2\end{array}$ & $\begin{array}{l}.980 \\
.956 \\
.914 \\
.852 \\
.770 \\
.678 \\
.573\end{array}$ & & $\begin{array}{l}\text { 1.3, }-2 \\
1.0,-2 \\
7.5,-3 \\
\text { 5. } 6,-3 \\
4.2,-3 \\
3.2,-3\end{array}$ & $\begin{array}{r}.365 \\
.282 \\
.205 \\
.140 \\
.088 \\
.051\end{array}$ \\
\hline .60 & $\begin{array}{l}1.0,-1 \\
7.5,-2 \\
5.6,-2 \\
4.2,-2 \\
3.2,-2 \\
2.4,-2 \\
1.8,-2 \\
1.3,-2 \\
1.0,-2\end{array}$ & $\begin{array}{l}.973 \\
.935 \\
.870 \\
.782 \\
.684 \\
.575 \\
.468 \\
.358 \\
.280\end{array}$ & .80 & $\begin{array}{l}\text { 7. } 5,-2 \\
\text { 5. } 6,-2 \\
\text { 4. } 2,-2 \\
\text { 3.2, } 2 \\
2.4,-2 \\
1.8,-2 \\
1.3,-2 \\
\text { 1. } 0,-2 \\
7.5,-3 \\
\text { 5. } 6,-3\end{array}$ & $\begin{array}{l}.989 \\
.963 \\
.910 \\
.831 \\
.723 \\
.599 \\
.456 \\
.348 \\
.244 \\
.158\end{array}$ \\
\hline .70 & $\begin{array}{l}\text { 1. } 0,-1 \\
7.5,-2\end{array}$ & $\begin{array}{l}.990 \\
.966\end{array}$ & & $\begin{array}{l}\text { 7. } 2,-3 \\
2.4,-3\end{array}$ & $\begin{array}{l}.050 \\
.052 \\
.024\end{array}$ \\
\hline
\end{tabular}

$\theta_{0}=30^{\circ} ; \frac{\theta}{\theta_{0}}=0.35$

[P1. 7]

\begin{tabular}{|c|c|c|c|c|c|}
\hline 0.10 & $\begin{array}{l}\text { 1. } 8,-1 \\
\text { 1. } 3,-1 \\
\text {. } 0,-1\end{array}$ & $\begin{array}{r}0.986 \\
.969 \\
.945\end{array}$ & 0.60 & $\begin{array}{l}2.4,-2 \\
1.8,=2 \\
1.3,=2 \\
1.0,=2\end{array}$ & $\begin{array}{r}0.532 \\
.415 \\
.297 \\
.216\end{array}$ \\
\hline $\begin{array}{l}.15 \\
.20 \\
.25 \\
.30 \\
.35\end{array}$ & $\begin{array}{l}1.8,-1 \\
1.8,-1 \\
1.8,-1 \\
1.8,-1 \\
1.8,-1\end{array}$ & $\begin{array}{l}.986 \\
.986 \\
.986 \\
.986 \\
.986\end{array}$ & .70 & $\begin{array}{l}1 . \\
1.0,-1 \\
7.5,-2 \\
5.6,-2 \\
4.2,-2\end{array}$ & $\begin{array}{r}.989 \\
.962 \\
.906 \\
.815\end{array}$ \\
\hline .40 & $\begin{array}{l}1.8,-1 \\
1.3,-1\end{array}$ & $\begin{array}{r}.988 \\
.970\end{array}$ & & & $\begin{array}{r}.568 \\
.435 \\
\end{array}$ \\
\hline .45 & $\begin{array}{l}1.3,-1 \\
1.0,-1 \\
7.5,-2 \\
5.6,-2\end{array}$ & $\begin{array}{l}.973 \\
.947 \\
.902 \\
.835\end{array}$ & & $\begin{array}{l}1.0,-2 \\
7.5,-3 \\
5.6,-3 \\
4.2,-3\end{array}$ & $\begin{array}{l}.218 \\
.144 \\
.087 \\
.047\end{array}$ \\
\hline .50 & $\begin{array}{l}1.3,-1 \\
1.0,-1 \\
7.5,-2 \\
5.6,-2 \\
4.2,-2 \\
3.2,-2 \\
2.4,-2\end{array}$ & $\begin{array}{l}.978 \\
.952 \\
.906 \\
.837 \\
.747 \\
.646 \\
.630\end{array}$ & .80 & $\begin{array}{l}7.5,-2 \\
5.6,-2 \\
4.2,-2 \\
3.2,-2 \\
2.4,-2 \\
1.8,-2 \\
1.3,-2\end{array}$ & $\begin{array}{l}.988 \\
.985 \\
.800 \\
.814 \\
.695 \\
.5559 \\
.403\end{array}$ \\
\hline .60 & $\begin{array}{l}1.0,-1 \\
7.5,-2 \\
5.6,-2 \\
4.2,-2 \\
3.2,-2\end{array}$ & $\begin{array}{l}.971 \\
.928 \\
.856 \\
.760 \\
.652\end{array}$ & & $\begin{array}{l}1.0,-2 \\
7.5,-3 \\
5.6,-3 \\
4.2,-3 \\
3.2,-3\end{array}$ & $\begin{array}{l}.889 \\
.185 \\
.06 \\
.054 \\
.025\end{array}$ \\
\hline
\end{tabular}

$\theta_{0}=30^{\circ} ; \frac{\theta}{\theta_{0}}=0.40$

[P1. 8]

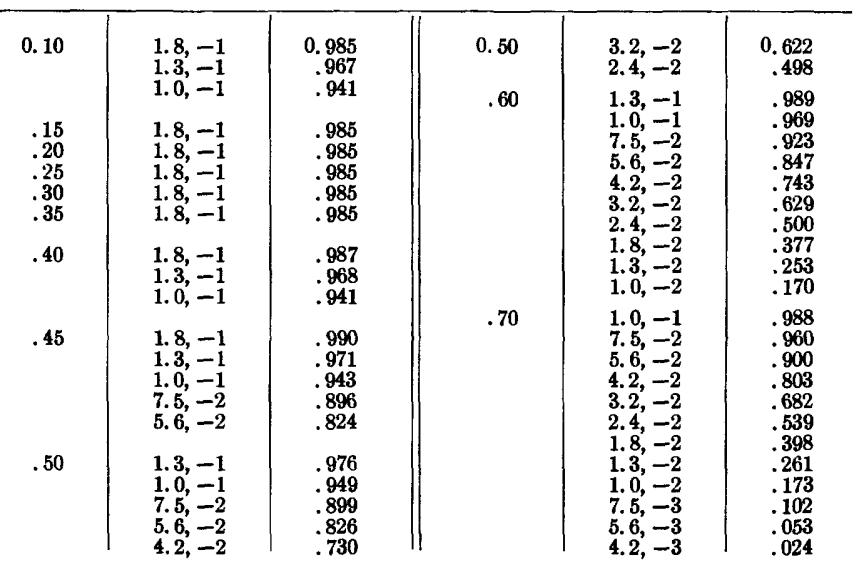

\begin{tabular}{|c|c|c|c|c|c|}
\hline$r / a$ & $\frac{T t}{r^{2} s}$ & $1-\frac{8}{80}$ & $r / a$ & $\frac{T t}{r^{2} s}$ & $1-\frac{8}{80}$ \\
\hline \multicolumn{6}{|c|}{$\theta_{0}=30^{\circ} ; \frac{\theta}{\theta_{0}}=0.40-$ Continued } \\
\hline 0.80 & $\begin{array}{l}7.5,-2 \\
5.6,-2 \\
4.2,-2 \\
3.2,-2 \\
2.4,-2 \\
1.8,-2\end{array}$ & $\begin{array}{r}0.987 \\
.956 \\
.894 \\
.802 \\
.674 \\
.529\end{array}$ & 0.80 & $\begin{array}{l}1.3,-2 \\
1.0,-2 \\
7.5,-3 \\
5.6,-3 \\
4.2,-3\end{array}$ & $\begin{array}{r}0.365 \\
.248 \\
.145 \\
.073 \\
.031\end{array}$ \\
\hline \multicolumn{6}{|c|}{$\begin{array}{c}\theta_{0}=30^{\circ} ; \frac{\theta}{\theta_{0}}=0.45 \\
{[\mathrm{P} 1.9]}\end{array}$} \\
\hline 0.10 & $\begin{array}{l}1.8,-1 \\
1.3,-1 \\
1.0,-1\end{array}$ & $\begin{array}{r}0.984 \\
.965 \\
.938\end{array}$ & 0.60 & $\begin{array}{l}\text { 5. } 6,-2 \\
4.2,-2 \\
3.2,-2\end{array}$ & $\begin{array}{r}0.841 \\
.734 \\
.614\end{array}$ \\
\hline $\begin{array}{l}.15 \\
.20 \\
.25\end{array}$ & $\begin{array}{l}1.8,-1 \\
1.8,-1 \\
1.8,-1\end{array}$ & $\begin{array}{l}.984 \\
.984 \\
.984 \\
.084\end{array}$ & & $\begin{array}{l}\text { 1. } 8,-2 \\
1.3,-2 \\
1.0,-2\end{array}$ & $\begin{array}{l}.353 \\
.226 \\
.143\end{array}$ \\
\hline .35 & $\begin{array}{l}1.8,-1 \\
1.8,-1\end{array}$ & .985 & .70 & $1.0,-1$ & .988 \\
\hline .40 & $\begin{array}{l}1.8,-1 \\
1.3,-1 \\
1.0,-1\end{array}$ & $\begin{array}{l}.986 \\
.967 \\
.939\end{array}$ & & $\begin{array}{l}7.0,-2 \\
5.6,=2 \\
4.2,=2 \\
3.2,=2\end{array}$ & $\begin{array}{l}.880 \\
.796 \\
.795 \\
.670\end{array}$ \\
\hline .45 & $\begin{array}{l}1.8,-1 \\
1.3,-1 \\
1.0,-1 \\
7.5,-2 \\
5.6,-2\end{array}$ & $\begin{array}{l}.989 \\
.970 \\
.941 \\
.892 \\
.817\end{array}$ & & $\begin{array}{l}1.8,-2 \\
1.3,-2 \\
1.0,-2 \\
7.5,-3 \\
5.6,-3\end{array}$ & $\begin{array}{l}.375 \\
.234 \\
.146 \\
.078 \\
.035\end{array}$ \\
\hline .50 & $\begin{array}{l}1.3,-1 \\
1.0,-1 \\
7.5,-2 \\
5.6,-2 \\
4.2,-2 \\
3.2,-2 \\
2.4,-2\end{array}$ & $\begin{array}{l}.975 \\
.947 \\
.895 \\
.819 \\
.720 \\
.607 \\
.479\end{array}$ & .80 & $\begin{array}{l}7.5,-2 \\
5.6,-2 \\
4.2,-2 \\
3.2,-2 \\
2.4,-2 \\
1.8,-2 \\
1.3,-2\end{array}$ & $\begin{array}{l}.986 \\
.954 \\
.890 \\
.794 \\
.662 \\
.511 \\
.342\end{array}$ \\
\hline .60 & $\begin{array}{l}1.3,-1 \\
1.0,-1 \\
7.5,-2\end{array}$ & $\begin{array}{l}.989 \\
.968 \\
.920\end{array}$ & & $\begin{array}{l}7.5,-3 \\
5.6,-3 \\
4.2,-3\end{array}$ & $\begin{array}{l}. .21 \\
.055 \\
.020\end{array}$ \\
\hline
\end{tabular}

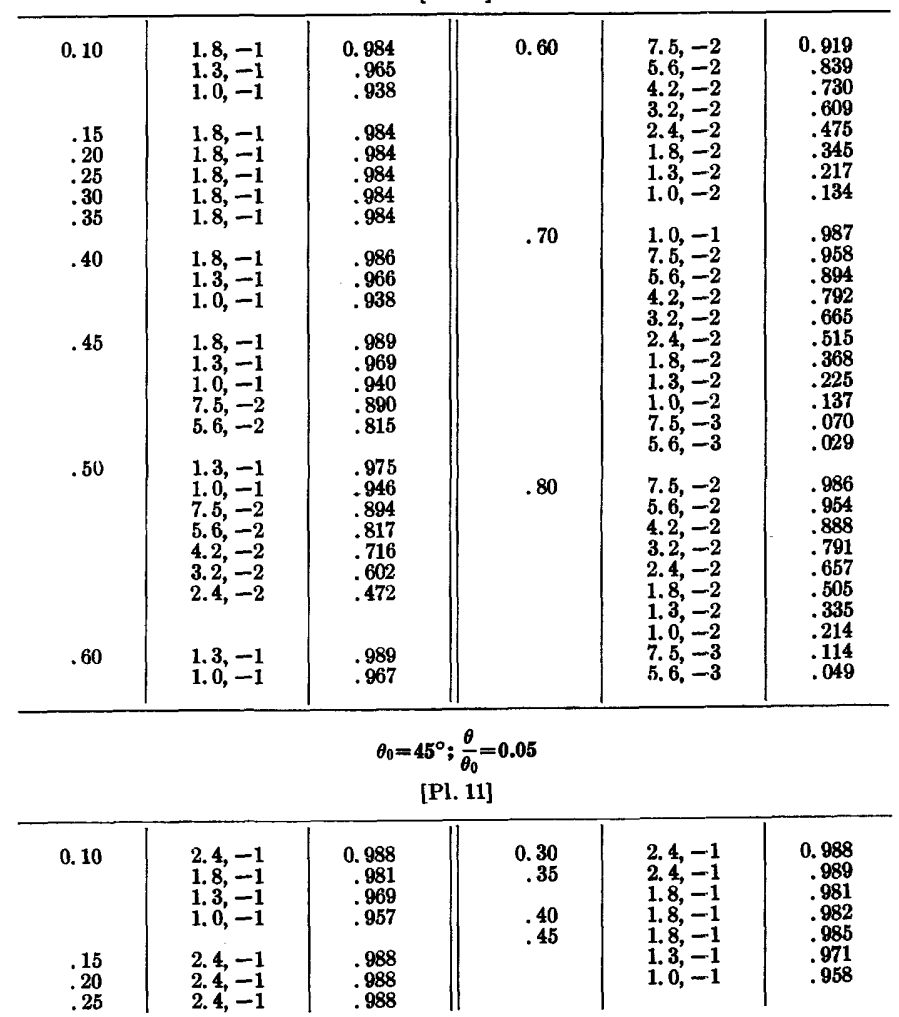




\begin{tabular}{|c|c|c|c|c|c|c|c|c|c|c|c|}
\hline$\tau / a$ & $\frac{T t}{r^{2} 8}$ & $1-\frac{8}{80}$ & $r / a$ & $\frac{T t}{r^{2} g}$ & $1-\frac{8}{80}$ & $r / a$ & $\frac{T_{t}}{r^{2}}$ & $1-\frac{8}{80}$ & $r / a$ & $\frac{T t}{r^{2}}$ & $1-\frac{8}{80}$ \\
\hline \multicolumn{6}{|c|}{$\theta_{0}=45^{\circ} ; \frac{\theta}{\theta_{0}}=0.05-$ Continued } & \multicolumn{6}{|c|}{$\theta_{0}=45^{\circ} ; \frac{\theta}{\theta_{0}}=0.15-$ Continued } \\
\hline 0.50 & $\begin{array}{l}1.8,-1 \\
1.3,-1 \\
1.0,-1 \\
7.5,-2 \\
5.6,-2 \\
4.2,-2 \\
3.2,-2\end{array}$ & $\begin{array}{r}0.988 \\
.975 \\
.960 \\
.942 \\
.921 \\
.900 \\
.880\end{array}$ & 0.70 & \multirow{3}{*}{$\begin{array}{l}2.4,-2 \\
1.8,=2 \\
1.3,-2 \\
1.0,-2 \\
7.5,-3 \\
5.6,-3 \\
4.2,-3 \\
7.5,-2 \\
5.6,-2 \\
4.2,-2 \\
3.2,-2 \\
2.4,-2 \\
1.8,-2 \\
1.3,-2 \\
1.0,-2 \\
7.5,-3 \\
5.6,-3 \\
4.2,-3 \\
3.2,-3 \\
2.4,-3 \\
1.8,-3 \\
1.3,-3\end{array}$} & \multirow{3}{*}{$\begin{array}{r}0.869 \\
.842 \\
.810 \\
.782 \\
.749 \\
.711 \\
.668 \\
.886 \\
.972 \\
.953 \\
.931 \\
.905 \\
.875 \\
.837 \\
.803 \\
.762 \\
.717 \\
.671 \\
.624 \\
.571 \\
.513 \\
.441\end{array}$} & 0.70 & \multirow{2}{*}{$\begin{array}{l}1.3,-1 \\
1.0,=1 \\
7.5,-2 \\
5.6,-2 \\
4.2,-2 \\
3.2,-2 \\
.4,-2 \\
1.8,-2 \\
1.3,-2 \\
1.0,-2 \\
7.5,-3 \\
5.6,-3 \\
4.2,-3 \\
3.2,-3\end{array}$} & $\begin{array}{r}0.981 \\
.965 \\
.910 \\
.847 \\
.773 \\
.701 \\
.625\end{array}$ & 0.80 & \multirow{2}{*}{$\begin{array}{l}1.0,-1 \\
7.5,-2 \\
5.6,-2 \\
4.2,-2 \\
3.2,-2 \\
2.4,-2 \\
1.8,-2 \\
1.3,-2 \\
1.0,-2 \\
7.5,-3 \\
5.6,-3 \\
4.2,-3 \\
3.2,-3 \\
2.4,-3 \\
1.8,-3 \\
1.3,-3\end{array}$} & \multirow{2}{*}{$\begin{array}{r}0.984 \\
.959 \\
.918 \\
.862 \\
.800 \\
.727 \\
. .446 \\
.547 \\
.462 \\
.371 \\
.283 \\
.206 \\
.144 \\
.0000 \\
.050 \\
.021\end{array}$} \\
\hline .60 & $\begin{array}{l}1.3,-1 \\
1.0,-1 \\
7.5,-2 \\
5.6,-2 \\
4.2,-2 \\
3.2,-2 \\
2.4,-2 \\
1.8,-2 \\
1.3,-2\end{array}$ & $\begin{array}{l}.985 \\
.971 \\
.951 \\
.928 \\
.904 \\
.882 \\
.859 \\
.836 \\
.808\end{array}$ & \multirow[t]{2}{*}{.80} & & & & & $\begin{array}{l}.552 \\
.472 \\
.408 \\
.338 \\
.267 \\
.200 \\
.142\end{array}$ & & & \\
\hline .70 & $\begin{array}{l}1.0,-1 \\
7 ., 5=2 \\
5.6,-2 \\
4.2,2 \\
3.2,-2\end{array}$ & $\begin{array}{l}.985 \\
.969 \\
.947 \\
.922 \\
.896\end{array}$ & & & & \multicolumn{6}{|c|}{$\begin{array}{c}\theta_{0}=45^{\circ} ; \frac{\theta}{\theta_{0}}=0.20 \\
{[\text { P1. 14] }}\end{array}$} \\
\hline \multicolumn{6}{|c|}{$\begin{array}{c}\theta_{0}=45^{\circ} ; \frac{\theta}{\theta_{0}}=0.10 \\
{[\text { Pl. 12] }}\end{array}$} & $\begin{array}{r}0.05 \\
.10 \\
.15\end{array}$ & $\begin{array}{l}5.6,-1 \\
4.2,-1 \\
5.6,-1 \\
5.6,-1\end{array}$ & $\begin{array}{r}0.990 \\
.982 \\
.990 \\
.990\end{array}$ & \multirow[t]{2}{*}{0.60} & \multirow{2}{*}{$\begin{array}{l}1.8,-1 \\
1.3,-1 \\
1.0,-1 \\
7.5,-2 \\
5,6,-2 \\
4.2,-2 \\
3.2,-2 \\
2.4,-2 \\
1.8,-2 \\
1.3,-2 \\
1.0,-2\end{array}$} & \multirow{2}{*}{$\begin{array}{r}0.979 \\
.942 \\
.891 \\
.817 \\
.730 \\
.641 \\
.561 \\
.484 \\
.412 \\
.332 \\
.269\end{array}$} \\
\hline $\begin{array}{l}0.10 \\
.15 \\
.20 \\
.25 \\
.30\end{array}$ & $\begin{array}{l}\text { 3. } 2,-1 \\
\text { 3. } 2,-1 \\
\text { 3. } 2,-1 \\
\text { 3. } 2,-1 \\
\text { 3. } 2,-1\end{array}$ & $\begin{array}{l}0.985 \\
.985 \\
.985 \\
.985 \\
.986\end{array}$ & 0.60 & $\begin{array}{l}3.2,-2 \\
2.4,-2 \\
1.8,-2 \\
1.3,-2 \\
1.0,-2\end{array}$ & $\begin{array}{r}0.767 \\
.724 \\
.680 \\
.627 \\
.579\end{array}$ & .20 & $\begin{array}{l}\text { 5. } 6,-1 \\
4.2,-1 \\
4.2,-1\end{array}$ & $\begin{array}{l}.990 \\
.982 \\
.983\end{array}$ & & & \\
\hline .35 & $\begin{array}{l}3.2,-1 \\
2.4,-1 \\
1.8,-1\end{array}$ & $\begin{array}{l}.988 \\
.977 \\
.963\end{array}$ & \multirow[t]{3}{*}{.70} & \multirow{3}{*}{$\begin{array}{l}1.3,-1 \\
1.0,-1 \\
7.5,-2 \\
5.6,-2 \\
4.2,-2 \\
3.2,-2 \\
2.4,-2 \\
1.8,-2 \\
1.3,-2 \\
1.0,-2 \\
7.5,-3 \\
5.6,-3 \\
4.2,-3 \\
3.2,-3\end{array}$} & \multirow{3}{*}{$\begin{array}{l}.987 \\
.970 \\
.939 \\
.896 \\
.846 \\
.796 \\
.743 \\
.691 \\
.631 \\
.580 \\
.522 \\
.459 \\
.392 \\
.327\end{array}$} & .30 & $\begin{array}{l}4.2,-1 \\
3.2,-1 \\
2.4,-1\end{array}$ & $\begin{array}{l}.985 \\
.973 \\
.955\end{array}$ & \multirow[t]{3}{*}{.70} & \multirow{3}{*}{$\begin{array}{l}1.3,-1 \\
1.0,-1 \\
7.5,-2 \\
5.6,-2 \\
4.2,-2 \\
3.2,-2 \\
2.4,-2 \\
1.8,-2 \\
1.3,-2 \\
1.0,-2 \\
7.5,-3 \\
5.6,-3 \\
4.2,-3 \\
3.2,-3\end{array}$} & \multirow{3}{*}{$\begin{array}{l}.975 \\
.942 \\
.883 \\
.801 \\
.707 \\
.614 \\
. .520 \\
. .431 \\
. .239 \\
.271 \\
.202 \\
.139 \\
.088 \\
.051\end{array}$} \\
\hline .40 & $\begin{array}{l}2.4,-1 \\
1.8,-1 \\
1.3,=1 \\
1.0,-1\end{array}$ & $\begin{array}{l}.980 \\
.965 \\
.941 \\
.915\end{array}$ & & & & .35 & $\begin{array}{l}4.2,-1 \\
3.2,=1 \\
2.4,-1 \\
1.8,-1 \\
1.3,-1\end{array}$ & $\begin{array}{l}.989 \\
.976 \\
. .987 \\
.829 \\
.885\end{array}$ & & & \\
\hline .45 & $\begin{array}{l}2.4,-1 \\
1.8,=1 \\
1.3,=1 \\
1.0,=1 \\
7.5,-2\end{array}$ & $\begin{array}{l}.985 \\
.969 \\
.944 \\
.917 \\
.883\end{array}$ & & & & .40 & $\begin{array}{l}3.2,-1 \\
2.4,=1 \\
1.8,=1 \\
1.3,=1 \\
1.0,=1\end{array}$ & $\begin{array}{l}.982 \\
.983 \\
.933 \\
.887 \\
.839\end{array}$ & & & \\
\hline .50 & $\begin{array}{l}2.4,-1 \\
1.8,-1 \\
1.3,-1 \\
1.0,-1 \\
7.5,-2 \\
5.6-2 \\
4.2,-2 \\
3.2,-2 \\
2.4,-2\end{array}$ & $\begin{array}{l}.990 \\
.9976 \\
.950 \\
.922 \\
.885 \\
.8403 \\
.803 \\
.723\end{array}$ & \multirow[t]{2}{*}{.80} & \multirow{2}{*}{$\begin{array}{l}1.0,-1 \\
7.5,-2 \\
5.6,-2 \\
4.2,-2 \\
3.2,-2 \\
2.4,-2 \\
1.8,-2 \\
1.3,-2 \\
1.0,-2 \\
7.5,-3 \\
5.6,-3 \\
4.2,-3 \\
3.2,-3 \\
2.4,-3 \\
1.8,-3 \\
1.3,-3\end{array}$} & \multirow{2}{*}{$\begin{array}{l}.989 \\
.972 \\
.944 \\
.906 \\
.864 \\
.813 \\
.756 \\
.683 \\
.620 \\
.546 \\
.471 \\
.397 \\
.328 \\
.258 \\
.191 \\
.124\end{array}$} & .45 & $\begin{array}{l}3.2,-1 \\
2.4,-1 \\
1.8,-1 \\
1.3,=1 \\
1.0,=1 \\
7.5,-2 \\
5.6,-2 \\
2.4,-1\end{array}$ & $\begin{array}{l}.989 \\
.971 \\
.942 \\
.894 \\
.842 \\
.777 \\
.903 \\
.981\end{array}$ & \multirow[t]{2}{*}{.80} & \multirow{2}{*}{$\begin{array}{l}1.0,-1 \\
7.5,-2 \\
5.6,-2 \\
4.2,-2 \\
3.2,-2 \\
2.4,-2 \\
1.8,-2 \\
1.3,-2 \\
1.0,-2 \\
7.5,-3 \\
5.6,-3 \\
4.2,-3 \\
3.2,-3 \\
2.4,--3\end{array}$} & \multirow{2}{*}{$\begin{array}{l}.979 \\
.947 \\
.893 \\
.822 \\
.742 \\
.648 \\
.549 \\
.431 \\
.337 \\
.241 \\
.158 \\
.095 \\
.052 \\
.024\end{array}$} \\
\hline .60 & $\begin{array}{l}1.8,-1 \\
1.3,=1 \\
1.0,=1 \\
7.5,=2 \\
5.6,=2 \\
4.2,-2\end{array}$ & $\begin{array}{l}.989 \\
.969 \\
.993 \\
.904 \\
.858 \\
.811\end{array}$ & & & & & $\begin{array}{l}1.8,-1 \\
1.3,-1 \\
1.0,-1 \\
7.5,-2 \\
5.6,-2 \\
4.2,-2 \\
3.2,-2 \\
2.4,-2\end{array}$ & $\begin{array}{l}.954 \\
.905 \\
.851 \\
.782 \\
.705 \\
.627 \\
.5555 \\
.482\end{array}$ & & & \\
\hline \multicolumn{6}{|c|}{$\begin{array}{c}\theta_{0}=45^{\circ} ; \frac{\theta}{\theta_{0}}=0.15 \\
{[\mathrm{Pl} .13]}\end{array}$} & \multirow{2}{*}{\multicolumn{6}{|c|}{$\begin{array}{c}\theta_{0}=45^{\circ} ; \frac{\theta}{\theta_{0}}=0.25 \\
{[\text { Pl. 15] }}\end{array}$}} \\
\hline \multirow{2}{*}{$\begin{array}{r}0.05 \\
.10 \\
.15 \\
.20 \\
.25\end{array}$} & 4. $2,-1$ & 0.986 & 0.45 & \multirow{4}{*}{$\begin{array}{l}7.5,-2 \\
5.6,-2 \\
2.4,-1 \\
1.8,-1 \\
1.3,-1 \\
1.0,-1 \\
7.5,-2 \\
5.6,-2 \\
4.2,-2 \\
3.2,-2 \\
2.4,-2\end{array}$} & \multirow{4}{*}{$\begin{array}{r}0.828 \\
.770 \\
.985 \\
.965 \\
.926 \\
.885 \\
.831 \\
.772 \\
.711 \\
.655 \\
.596\end{array}$} & & & & & & \\
\hline & $\begin{array}{l}4.2,-1 \\
4.2,-1 \\
4.2,-1 \\
4.2,-1\end{array}$ & $\begin{array}{l}.986 \\
.986 \\
.986 \\
.987\end{array}$ & .50 & & & 0.05 & $\begin{array}{l}5.6,-1 \\
4.2,-1\end{array}$ & $\begin{array}{r}0.987 \\
.979\end{array}$ & 0.40 & $\begin{array}{l}1.3,-1 \\
1.0,-1 \\
7.5,-2\end{array}$ & $\begin{array}{r}0.864 \\
.807 \\
.730\end{array}$ \\
\hline .30 & $\begin{array}{l}4.2,-1 \\
3.2,-1\end{array}$ & .988 & & & & $\begin{array}{l}.10 \\
.15 \\
.20\end{array}$ & $\begin{array}{l}\text { 5. } 6,-1 \\
\text { 5. } 6,-1 \\
\text { 5. } 6,-1\end{array}$ & $\begin{array}{l}.987 \\
.987 \\
.987\end{array}$ & .45 & $\begin{array}{l}3.2,-1 \\
2.4,-1\end{array}$ & $\begin{array}{l}.987 \\
.966\end{array}$ \\
\hline .35 & $\begin{array}{l}3.2,-1 \\
2.4,-1 \\
1.8,-1 \\
1.3,-1\end{array}$ & $\begin{array}{l}.982 \\
.967 \\
.945 \\
.912\end{array}$ & & & & .30 & $\begin{array}{l}5.6,-1 \\
4.2,-1 \\
4.2,-1 \\
\text { 3. } 2,-1\end{array}$ & $\begin{array}{l}.988 \\
.979 \\
.982\end{array}$ & & $\begin{array}{l}1.8,-1 \\
1.3,-1 \\
1.0,-1 \\
7.5,-2 \\
\text { 5. } 6,-2\end{array}$ & $\begin{array}{l}.930 \\
.871 \\
.810 \\
.732 \\
.643\end{array}$ \\
\hline .40 & $\begin{array}{l}3.2,-1 \\
2.4,-1 \\
1.8,=1 \\
1.3,-1 \\
1.0,-1\end{array}$ & $\begin{array}{l}.986 \\
.971 \\
.948 \\
.913 \\
.876\end{array}$ & .60 & $\begin{array}{l}1.8,-1 \\
1.3,-1 \\
1.0,=1 \\
7.5,-2 \\
5.6,=2 \\
4.2,-2\end{array}$ & $\begin{array}{l}.984 \\
. .955 \\
.916 \\
.859 \\
.791 \\
.722\end{array}$ & .35 & $\begin{array}{l}2.4,-1 \\
4.2,-1 \\
3.2,-1 \\
2.4,-1 \\
1.8,-1\end{array}$ & $\begin{array}{l}.946 \\
.986 \\
.972 \\
.948 \\
.915\end{array}$ & .50 & $\begin{array}{l}2.4,-1 \\
1.8,=1 \\
1.3,=1 \\
1.0,=1 \\
7.5,-2\end{array}$ & $\begin{array}{l}.977 \\
.945 \\
.885 \\
.821 \\
.737\end{array}$ \\
\hline .45 & $\begin{array}{l}2.4,-1 \\
1.8,-1 \\
1.3,=1 \\
1.0,-1\end{array}$ & $\begin{array}{l}.978 \\
.955 \\
.917 \\
.878\end{array}$ & & $\begin{array}{l}3.2,-2 \\
2.4,=2 \\
1.8,-2 \\
1.3,=2 \\
1.0,-2\end{array}$ & $\begin{array}{l}.660 \\
.598 \\
.557 \\
.466 \\
.406\end{array}$ & .40 & $\begin{array}{l}1.3,-1 \\
3.2,-1 \\
2.4,-1 \\
1.8,-1\end{array}$ & $\begin{array}{l}.862 \\
.979 \\
.955 \\
.920\end{array}$ & & $\begin{array}{l}\text { 5. } 6,-2 \\
4.2,-2 \\
3.2,-2 \\
2.4,-2\end{array}$ & $\begin{array}{l}.645 \\
.552 \\
.468 \\
.384\end{array}$ \\
\hline
\end{tabular}


TABLES

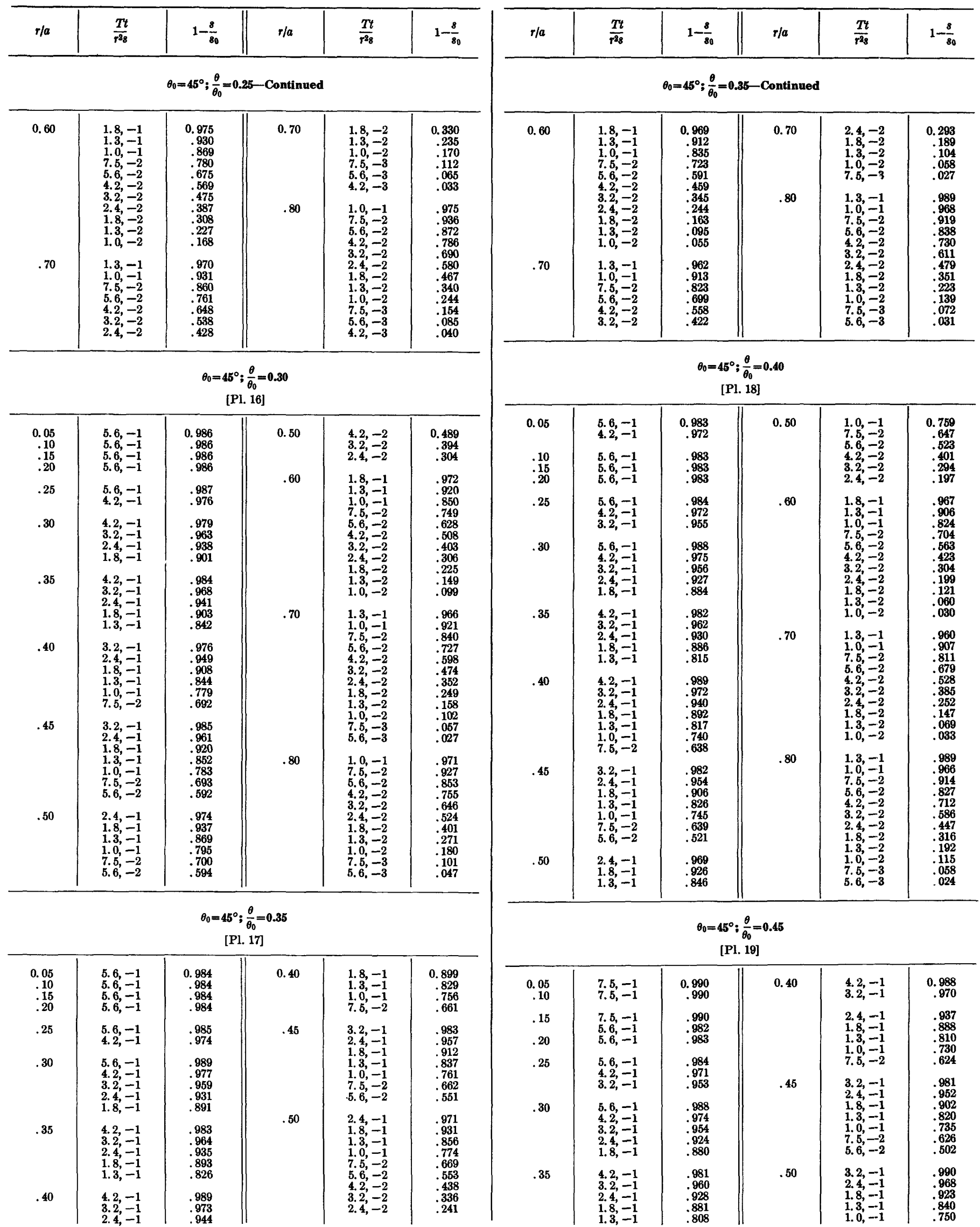


HYDRAULIC DIFFUSIVITY, WEDGE-SHAPED AQUIFERS DRAINED BY STREAMS

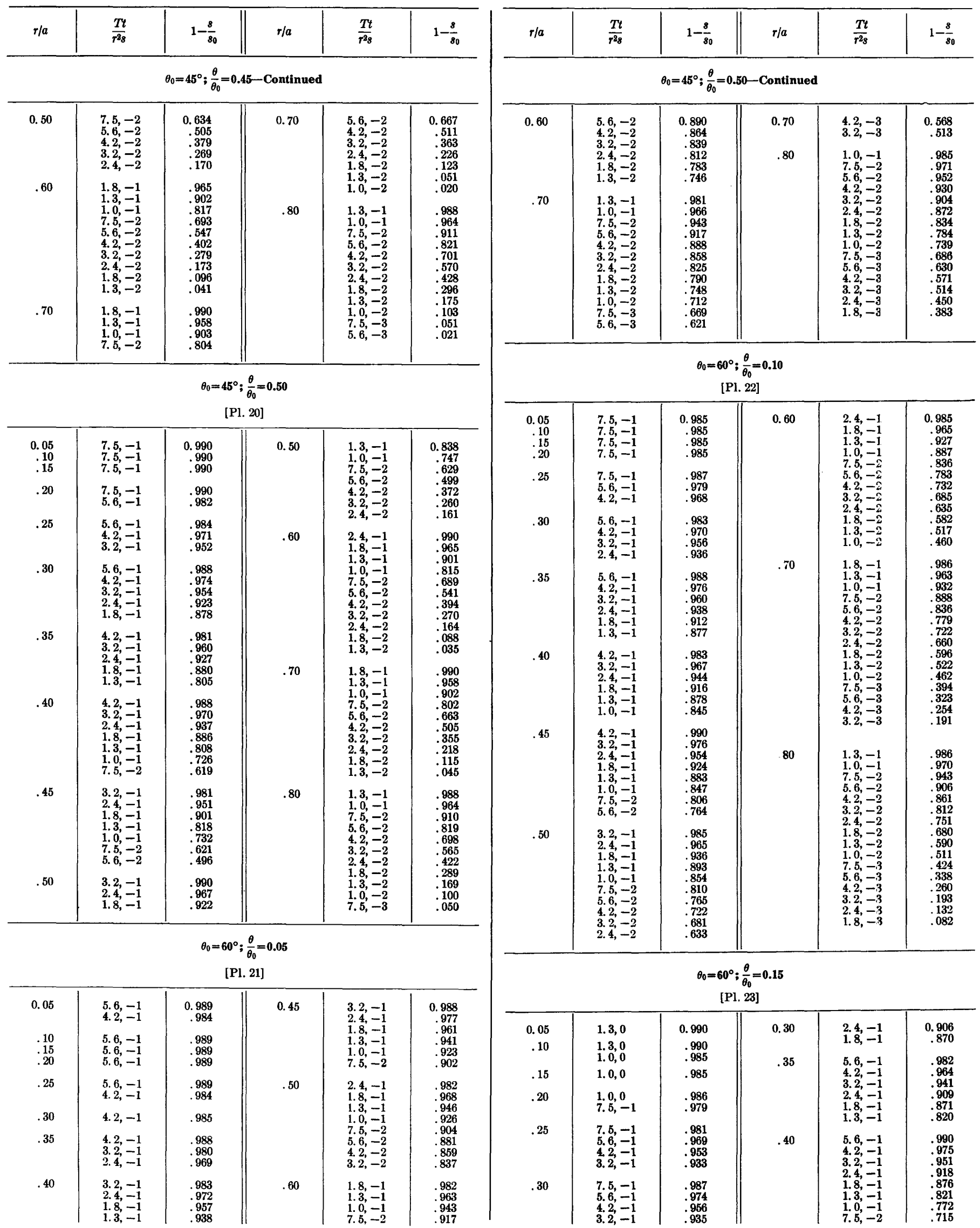


TABLES

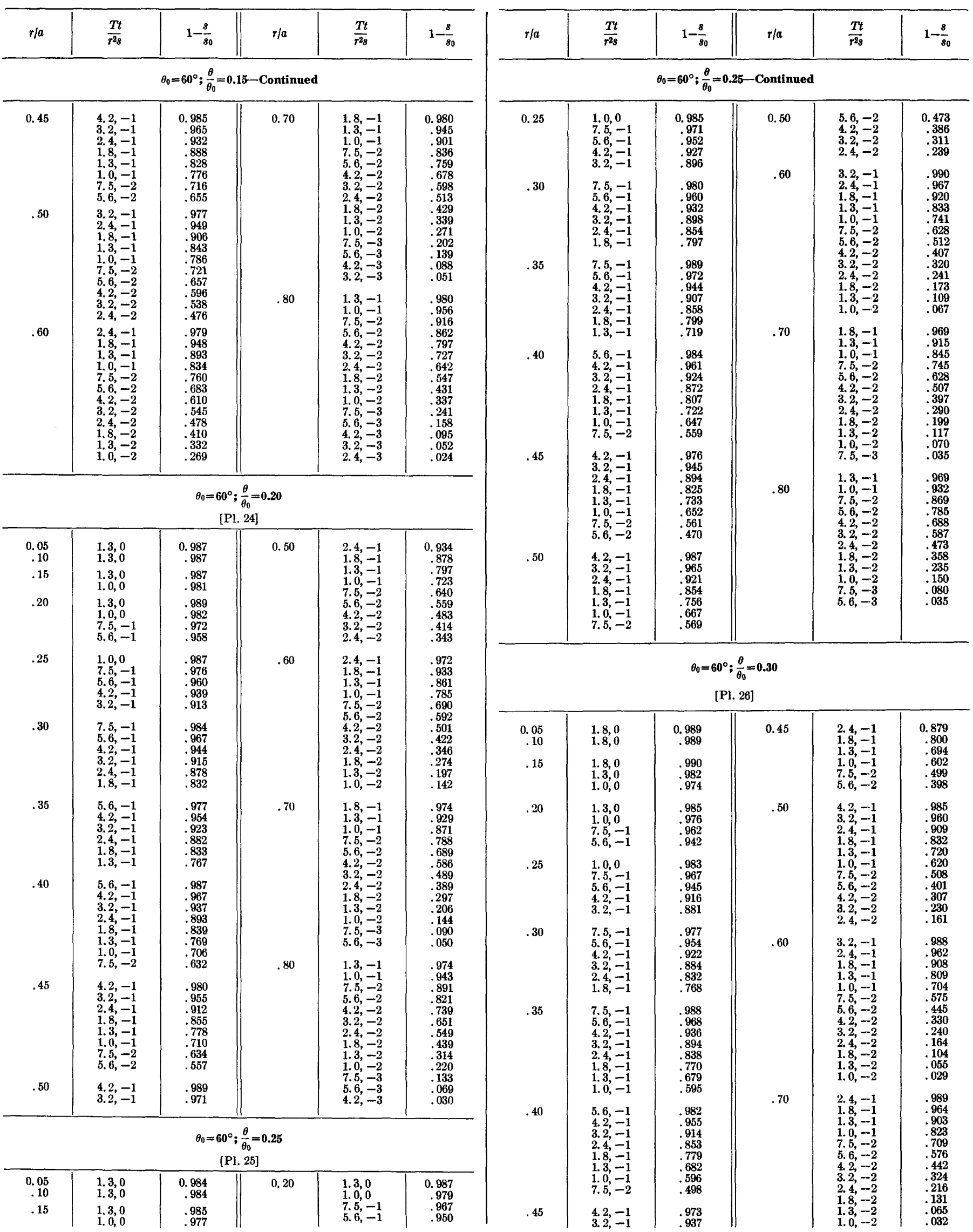




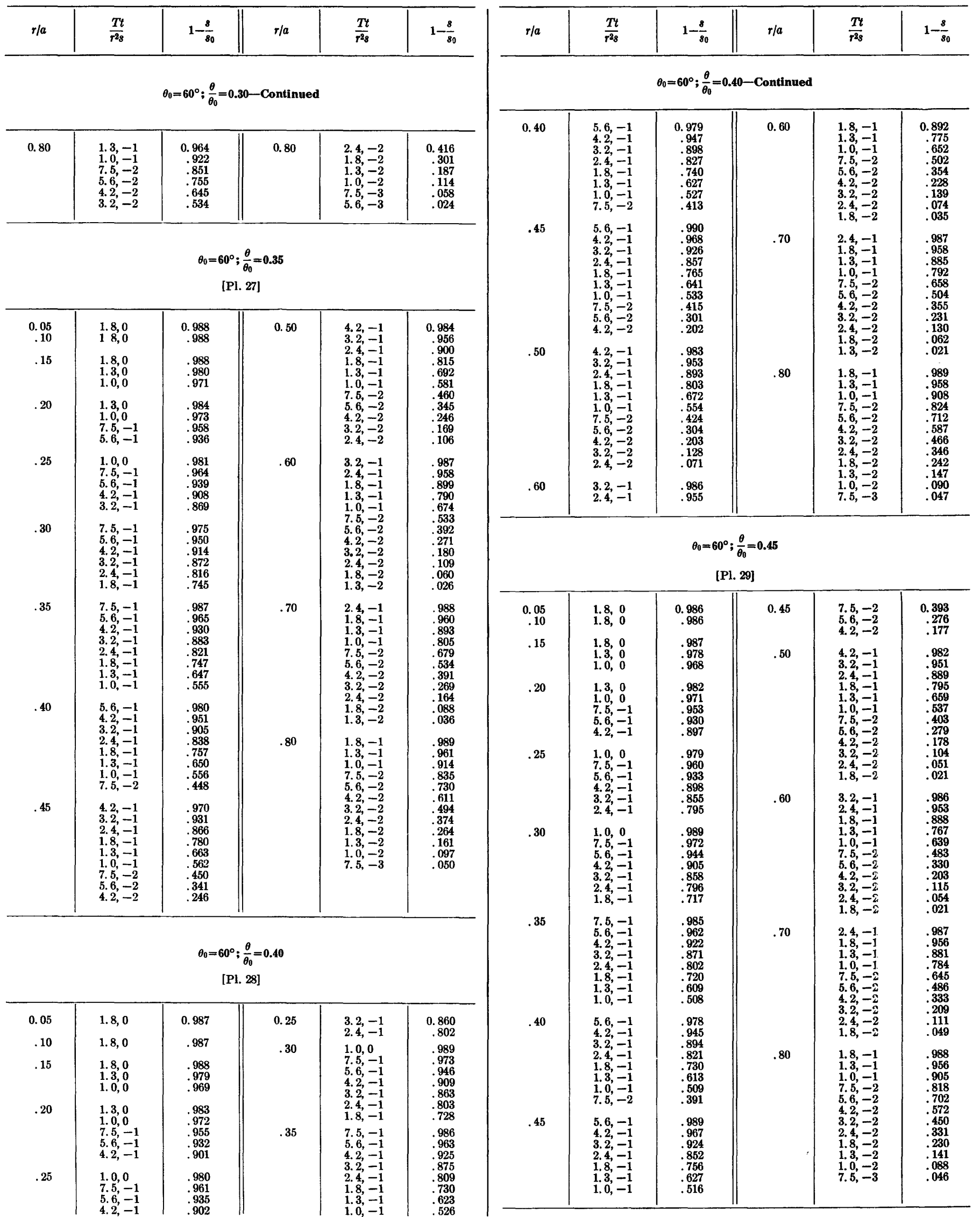




\begin{tabular}{|c|c|c|c|c|c|}
\hline$r / a$ & $\frac{T t}{r^{28}}$ & $1-\frac{8}{80}$ & $r / a$ & $\frac{T t}{r^{2} s}$ & $1-\frac{8}{80}$ \\
\hline \multicolumn{6}{|c|}{$\begin{array}{c}\theta_{0}=60^{\circ} ; \frac{\theta}{\theta_{0}}=0.50 \\
{[\text { Pl. 30] }}\end{array}$} \\
\hline $\begin{array}{r}0.05 \\
.10\end{array}$ & $\begin{array}{l}1.8,0 \\
1.8,0\end{array}$ & $\begin{array}{r}0.986 \\
.986\end{array}$ & \multirow[t]{2}{*}{0.45} & \multirow{2}{*}{$\begin{array}{l}1.0,-1 \\
7.5,-2 \\
5.6,-2 \\
4.2,-2\end{array}$} & \multirow{2}{*}{$\begin{array}{r}0.510 \\
.386 \\
.268 \\
.168\end{array}$} \\
\hline .15 & $\begin{array}{l}1.8,0 \\
1.3,0\end{array}$ & $\begin{array}{l}.987 \\
.978\end{array}$ & & & \\
\hline & $1.0,0$ & .968 & \multirow[t]{3}{*}{.50} & \multirow{3}{*}{$\begin{array}{l}4.2,-1 \\
3.2,-1 \\
2.4,-1 \\
1.8,-1 \\
1.3,-1 \\
1.0,-1 \\
7.5,-2 \\
5.6,-2 \\
4.2,-2 \\
3.2,-2 \\
2.4,-2\end{array}$} & \multirow{3}{*}{$\begin{array}{l}.982 \\
.985 \\
.888 \\
.793 \\
.655 \\
.531 \\
.396 \\
.721 \\
.169 \\
.096 \\
.045\end{array}$} \\
\hline .20 & $\begin{array}{l}1.3,0 \\
1.0,0 \\
7.5,-1 \\
5.6,-1 \\
4.2,-1\end{array}$ & $\begin{array}{l}.982 \\
.970 \\
.953 \\
.929 \\
.896\end{array}$ & & & \\
\hline .25 & $\begin{array}{l}1.0,0 \\
7.5,-1 \\
5.6,-1\end{array}$ & $\begin{array}{l}.979 \\
.959 \\
.932 \\
.897\end{array}$ & & & \\
\hline & $\begin{array}{l}\text { 3. } 2,-1 \\
2.4,-1\end{array}$ & $\begin{array}{l}.851 \\
.892\end{array}$ & \multirow[t]{2}{*}{.60} & \multirow{2}{*}{$\begin{array}{l}\text { 3. } 2,-1 \\
2.4,-1 \\
1.8,-1 \\
1.3,-1 \\
1.0,-1 \\
\text { 7. } 5,-2 \\
5.6,-2 \\
4.2,-2 \\
\text { 3. } 2,-2 \\
2.4,-2\end{array}$} & \multirow{2}{*}{$\begin{array}{l}.985 \\
.953 \\
.887 \\
.764 \\
.634 \\
.477 \\
.323 \\
.195 \\
.107 \\
.048\end{array}$} \\
\hline .30 & $\begin{array}{l}1.0,0 \\
7.5,-1 \\
5.6,-1 \\
4.2,-1 \\
3.2,-1 \\
2.4,-1 \\
1.8,-1\end{array}$ & $\begin{array}{l}.989 \\
.972 \\
.943 \\
.904 \\
.856 \\
.793 \\
.714\end{array}$ & & & \\
\hline .35 & $\begin{array}{l}7.5,-1 \\
5.6,-1 \\
4.2,-1 \\
3.2,-1 \\
2.4,-1 \\
1.8,-1 \\
1.3,-1 \\
1.0,-1\end{array}$ & $\begin{array}{l}.985 \\
.961 \\
.921 \\
.869 \\
.800 \\
.716 \\
.604 \\
.502\end{array}$ & \multirow[t]{2}{*}{.70} & \multirow[t]{2}{*}{$\begin{array}{l}2.4,-1 \\
1.8,-1 \\
\text { 1. } 3,-1 \\
\text { 1. } 0,-1 \\
\text { 7. } \\
\text { 5. } 6,-2 \\
\text { 4. } 2,-2 \\
\text { 3. } 2,-2 \\
\text { 2.4 } 4=2 \\
\text { 1. } 8,-2\end{array}$} & \multirow[t]{2}{*}{$\begin{array}{l}.987 \\
.956 \\
.880 \\
.781 \\
.641 \\
.480 \\
.326 \\
.202 \\
.105 \\
.045\end{array}$} \\
\hline .40 & 5. $6,-1$ & .978 & & & \\
\hline & $\begin{array}{l}\text { 3. } 2,-1 \\
2.4,-1 \\
1.8,-1 \\
1.3,-1 \\
\text { 1.0, } \\
\text { 7.5, }-2\end{array}$ & $\begin{array}{l}. .893 \\
.819 \\
.727 \\
.608 \\
.503 \\
.384\end{array}$ & \multirow[t]{2}{*}{.80} & \multirow{2}{*}{$\begin{array}{l}1.8,-1 \\
1.3,-1 \\
1.0,=1 \\
7.5,-2 \\
5.6,-2 \\
4.2,-2 \\
3.2,-2 \\
2.4,-2 \\
1.8,-2 \\
1.3,=2 \\
1.0,-2 \\
7.5,-3\end{array}$} & \multirow{2}{*}{$\begin{array}{l}.988 \\
.956 \\
.004 \\
.815 \\
.698 \\
. .668 \\
.442 \\
. .225 \\
.226 \\
.0089 \\
.046\end{array}$} \\
\hline .45 & $\begin{array}{l}\text { 5. } 6,-1 \\
\text { 4. } 2,-1 \\
\text { 3.2, }=1 \\
\text { 2. } 4,-1 \\
\text { 1. } 8,-1 \\
\text { 1. } 3,-1\end{array}$ & $\begin{array}{l}.989 \\
.966 \\
.923 \\
.850 \\
.753 \\
.623\end{array}$ & & & \\
\hline
\end{tabular}

$\theta_{0}=75^{\circ} ; \frac{\theta}{\theta_{0}}=0.05$

[Pl. 31]

\begin{tabular}{|c|c|c|c|c|c|}
\hline $\begin{array}{r}0.05 \\
.10 \\
.15\end{array}$ & $\begin{array}{l}1.0,0 \\
1.0,0 \\
1.0,0\end{array}$ & $\begin{array}{r}0.987 \\
.987 \\
.987\end{array}$ & \multirow[t]{3}{*}{0.50} & \multirow{3}{*}{$\begin{array}{l}1.8,-1 \\
1.3,-1 \\
1.0,-1 \\
7.5,-2 \\
5.6-2 \\
4.2,-2 \\
3.2,-2\end{array}$} & \multirow{3}{*}{$\begin{array}{r}0.946 \\
.919 \\
.897 \\
.872 \\
.847 \\
.822 \\
.796\end{array}$} \\
\hline .20 & $\begin{array}{l}1.0,0 \\
7.5,-1\end{array}$ & $\begin{array}{r}.988 \\
.983\end{array}$ & & & \\
\hline .25 & $\begin{array}{l}\text { 7. } 5,-1 \\
\text { 5. } 6,-1\end{array}$ & $\begin{array}{l}.984 \\
.977\end{array}$ & & & \\
\hline . 30 & $\begin{array}{l}\text { 4. } 2,-1 \\
\text { 7. } 5,-1 \\
\text { 5. } 6,-1 \\
\text { 4. } 2,-1 \\
\text { 3. } 2,-1\end{array}$ & $\begin{array}{l}.968 \\
.988 \\
.980 \\
.970 \\
.958\end{array}$ & .60 & $\begin{array}{l}2.4,-1 \\
1.8,-1 \\
1.3,-1 \\
1.0,-1 \\
7.5,-2\end{array}$ & $\begin{array}{l}.984 \\
.968 \\
.942 \\
.918 \\
.889 \\
.580\end{array}$ \\
\hline .35 & $\begin{array}{l}\text { 5. } 6,-1 \\
\text { 4. } 2,-1 \\
\text { 3. } 2,-1 \\
\text { 2. } 4,-1 \\
\text { 1. } 8,-1\end{array}$ & $\begin{array}{l}.985 \\
.974 \\
.961 \\
.946 \\
.930\end{array}$ & & $\begin{array}{l}0.0,-2 \\
4.2,-2 \\
3.2,-2 \\
2.4,-2 \\
1.8,-2 \\
1.3,-2\end{array}$ & $\begin{array}{l}.809 \\
.828 \\
.799 \\
.766 \\
.731 \\
.685\end{array}$ \\
\hline .40 & $\begin{array}{l}\text { 4. } 2,-1 \\
\text { 3. } 2,-1 \\
2.4,-1 \\
\text { 1. } 8,-1 \\
1.3,-1\end{array}$ & $\begin{array}{l}.980 \\
.967 \\
.950 \\
.932 \\
.910\end{array}$ & .70 & $\begin{array}{l}1.0,-2 \\
1.8,-1 \\
1.3,-1 \\
1.0,-1 \\
7.5,-2\end{array}$ & $\begin{array}{l}.047 \\
.985 \\
.968 \\
.948 \\
.922\end{array}$ \\
\hline 45 & $\begin{array}{l}\text { 4. } 2,-1 \\
3.2,-1 \\
2.4,-1 \\
1.8,-1 \\
1.3,-1 \\
1.0,-1 \\
7.5,-2\end{array}$ & $\begin{array}{l}.987 \\
.975 \\
.958 \\
.937 \\
.913 \\
.892 \\
.870\end{array}$ & & $\begin{array}{l}4.2,-2 \\
3.2,-2 \\
2.4,-2 \\
1.8,-2 \\
1.3,-2 \\
1.0,2 \\
7.5,-3\end{array}$ & $\begin{array}{l}.858 \\
.823 \\
.782 \\
.740 \\
.689 \\
.645 \\
.694 \\
.594\end{array}$ \\
\hline .50 & $\begin{array}{l}\text { 3. } 2,-1 \\
\text { 2. } 4,-1\end{array}$ & $\begin{array}{l}.982 \\
.967\end{array}$ & & & $\begin{array}{l}.437 \\
.414 \\
.414\end{array}$ \\
\hline
\end{tabular}

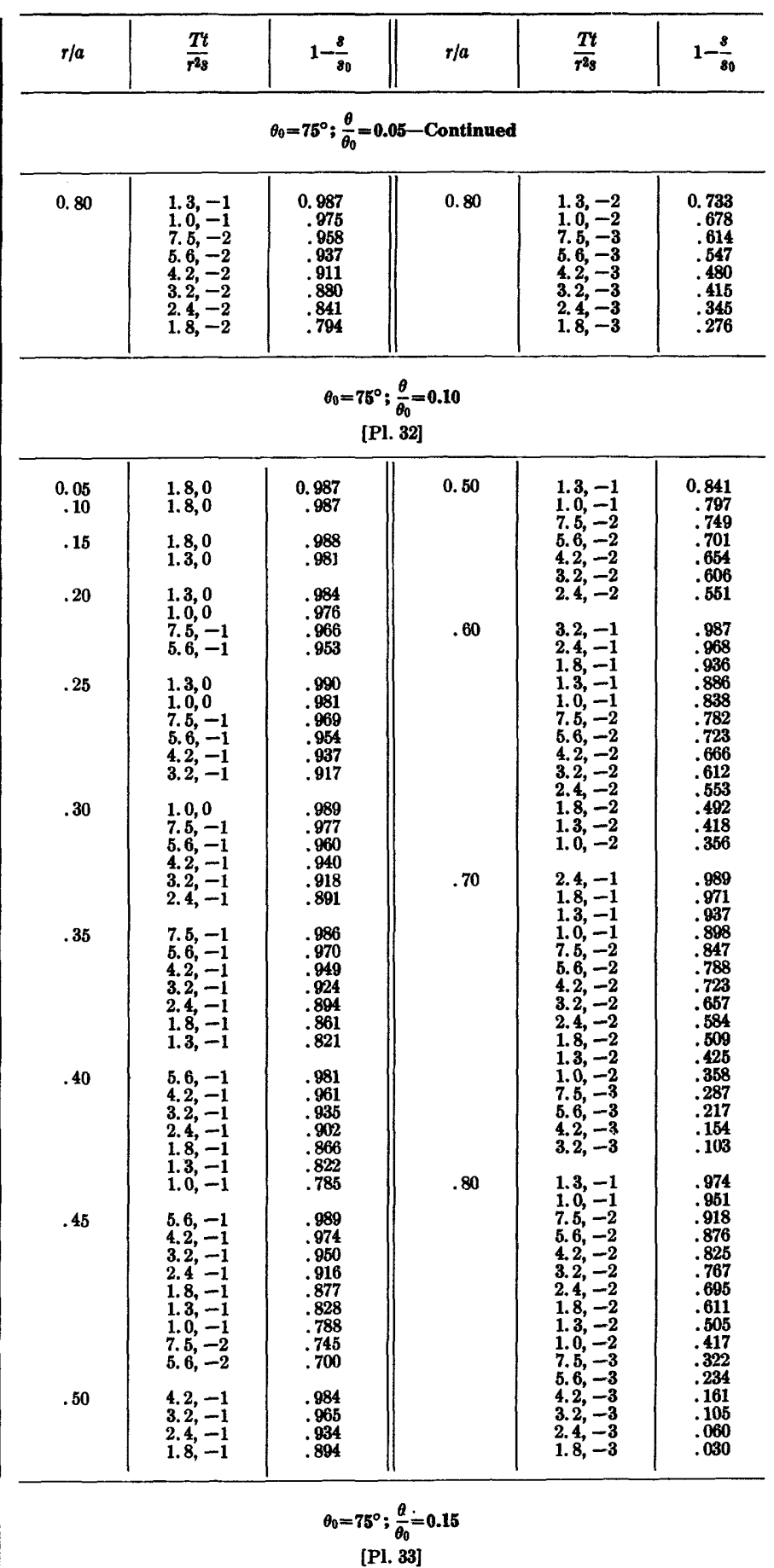

\begin{tabular}{|c|c|c|c|c|c|}
\hline 0.05 & $2.4,0$ & 0.986 & 0.25 & 5. $6,-1$ & $\begin{array}{l}0.933 \\
0977\end{array}$ \\
\hline .10 & $\begin{array}{l}2.4,0 \\
1.8,0\end{array}$ & .986 & \multirow{3}{*}{.30} & 3. $2,-1$ & .877 \\
\hline .15 & $\begin{array}{l}2.4,0 \\
1.8,0 \\
1.3,0 \\
1.0,0\end{array}$ & $\begin{array}{l}.988 \\
.982 \\
.973 \\
.963\end{array}$ & & $\begin{array}{l}7.5,-1 \\
5.6,-1 \\
4.2,-1 \\
3.2,-1\end{array}$ & $\begin{array}{r}.9660 \\
.942 \\
.912 \\
.880 \\
880\end{array}$ \\
\hline \multirow[t]{2}{*}{.20} & $\begin{array}{l}1.8,0 \\
1.3,0\end{array}$ & $\begin{array}{l}.987 \\
.976\end{array}$ & & 1. $8,-1$ & .795 \\
\hline & $\begin{array}{l}1.0,0 \\
7.5,-1 \\
5.6,-1\end{array}$ & $\begin{array}{l}.965 \\
.950 \\
.930\end{array}$ & \multirow[t]{2}{*}{.35} & $\begin{array}{l}\text { 7. 5, }-1 \\
\text { 5. 6, }=1 \\
4.2,-1\end{array}$ & $\begin{array}{l}.979 \\
.956 \\
.925 \\
888\end{array}$ \\
\hline .25 & $\begin{array}{l}1.3,0 \\
1.0,0 \\
7.5,-1\end{array}$ & $\begin{array}{l}.985 \\
.973 \\
.955\end{array}$ & & $\begin{array}{l}2.4,-1 \\
1.8,-1 \\
1.3,-1\end{array}$ & $\begin{array}{l}.844 \\
.797 \\
.737\end{array}$ \\
\hline
\end{tabular}




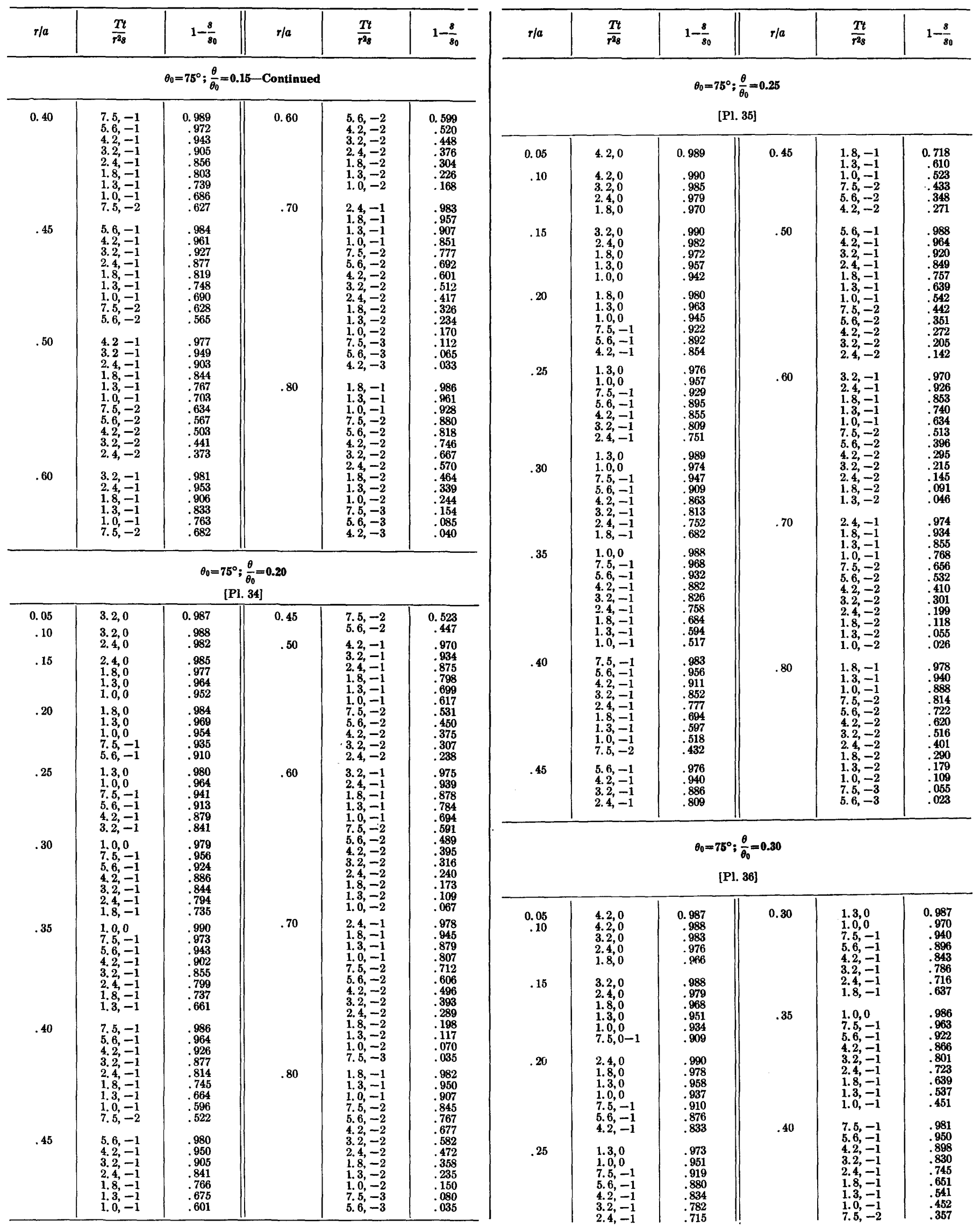




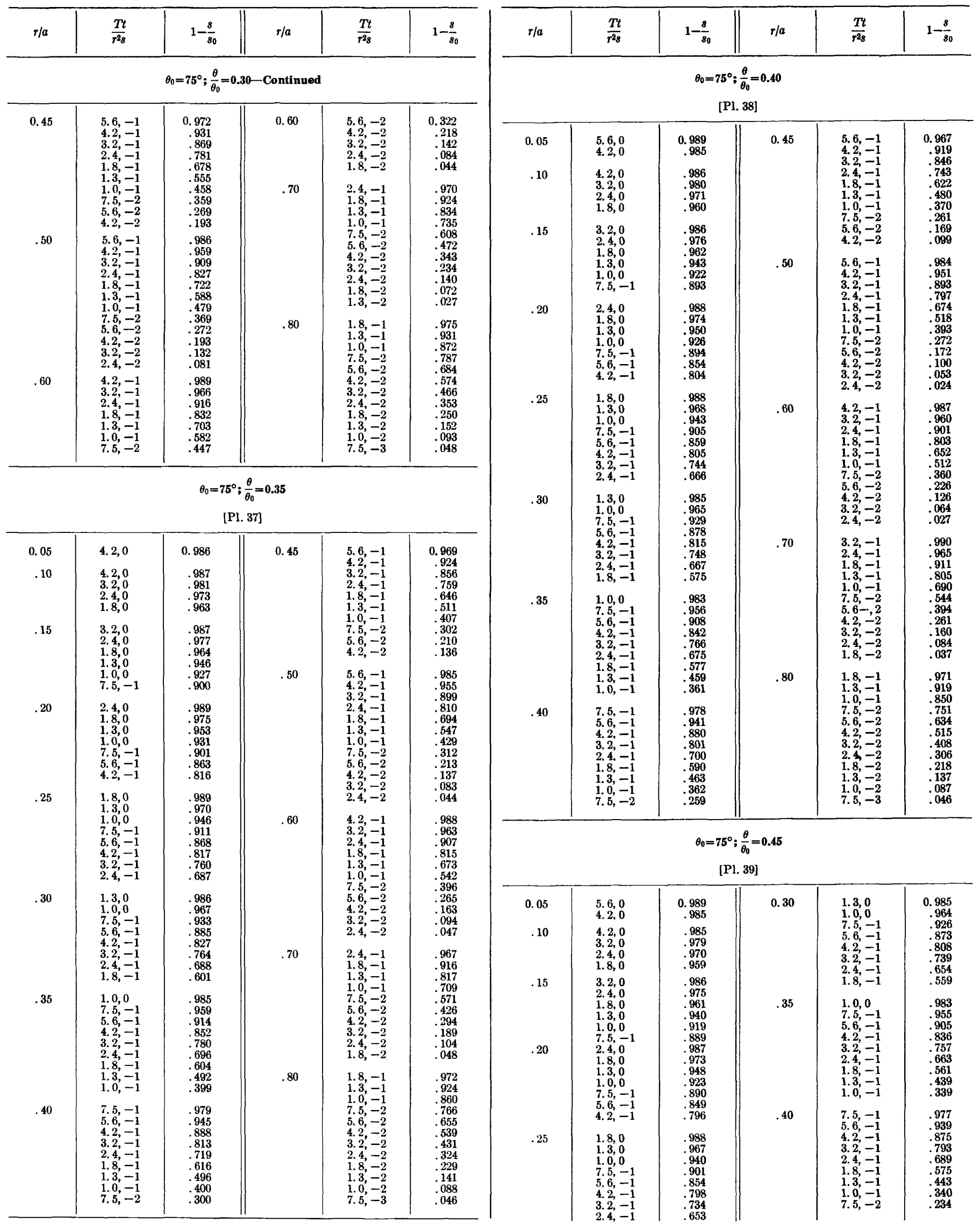


HYDRAULIC DIFFUSIVITY, WEDGE-SHAPED AQUIFERS DRAINED BY STREAMS

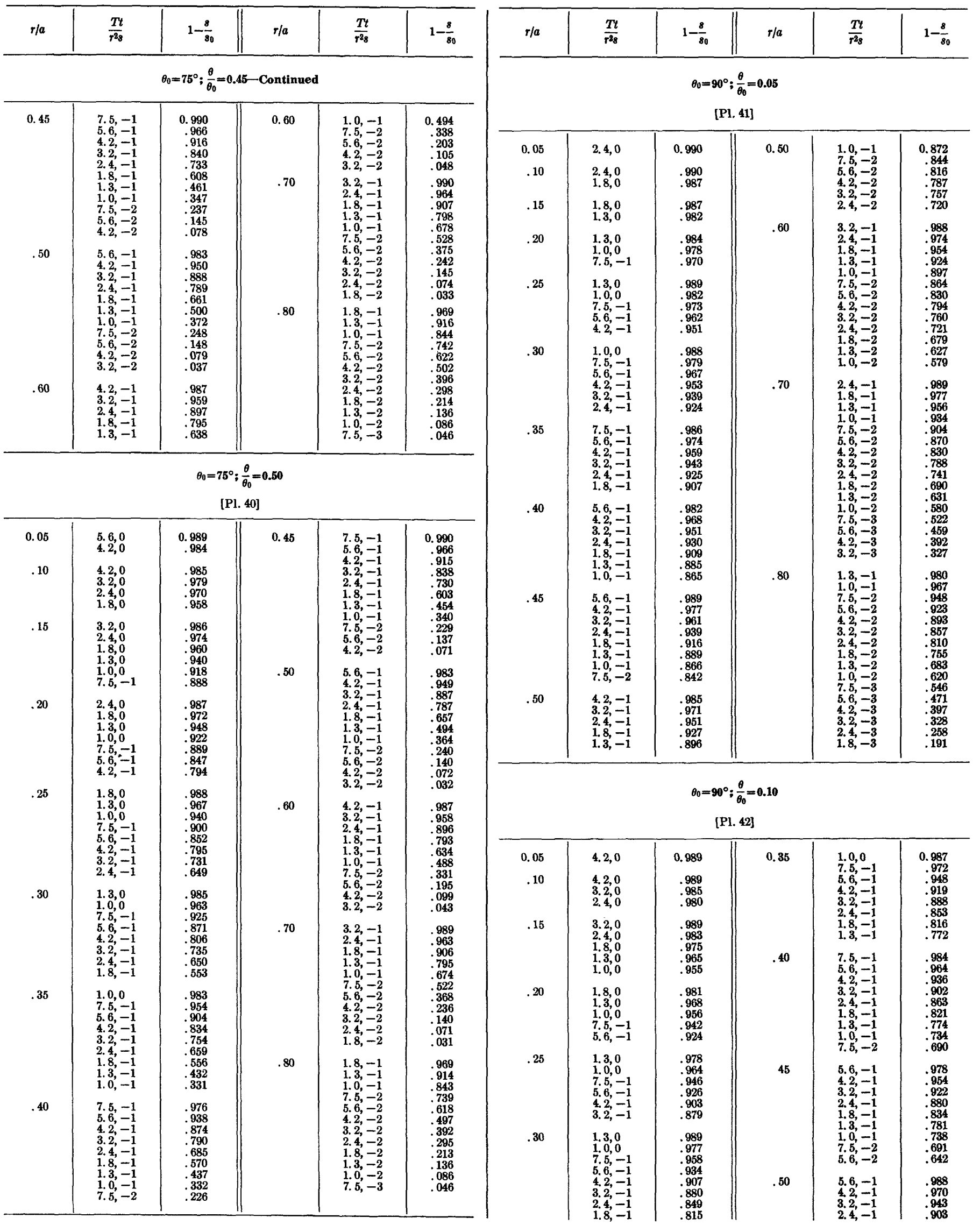




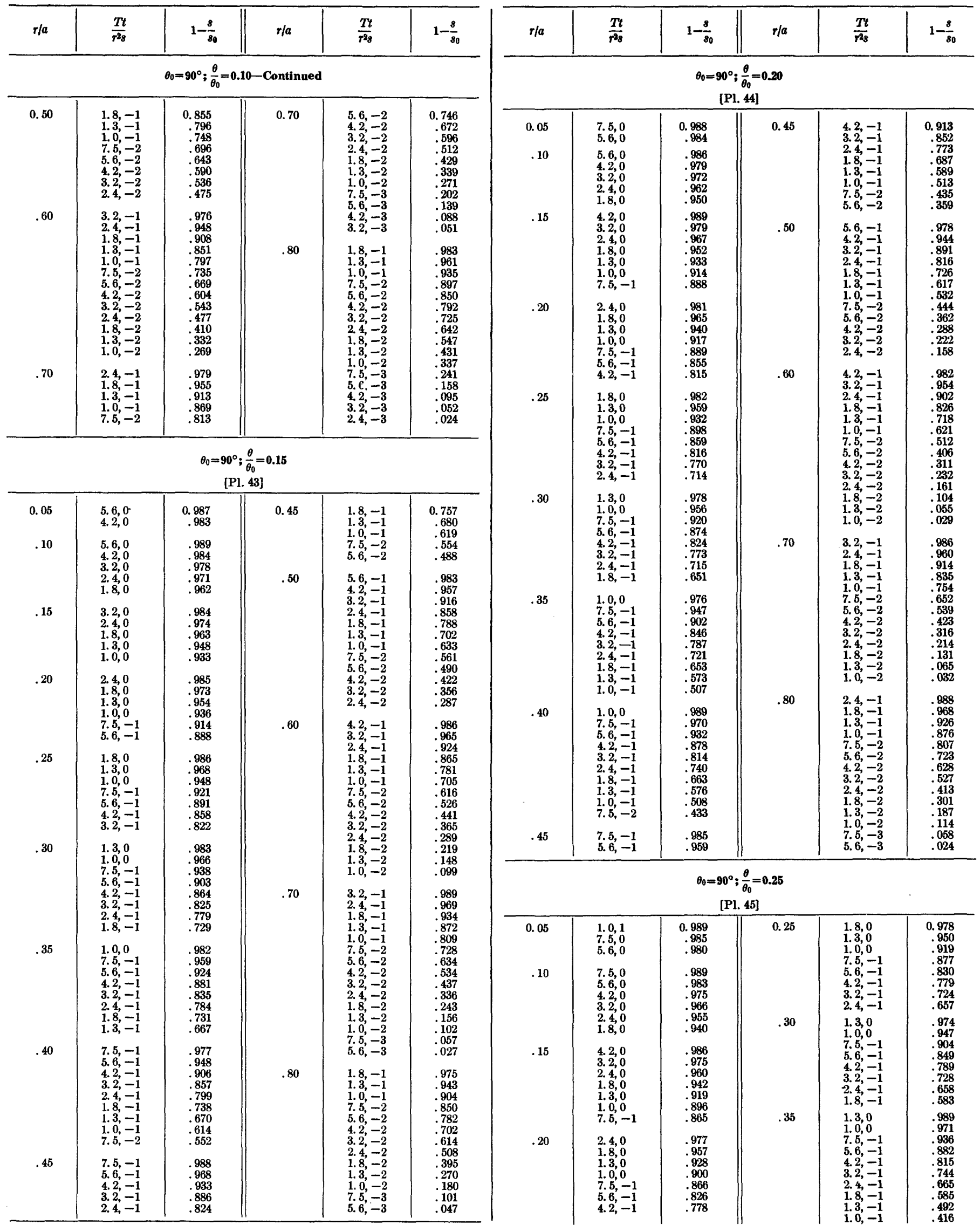


HYDRAULIC DIFFUSIVITY, WEDGE-SHAPED AQUIFERS DRAINED BY STREAMS

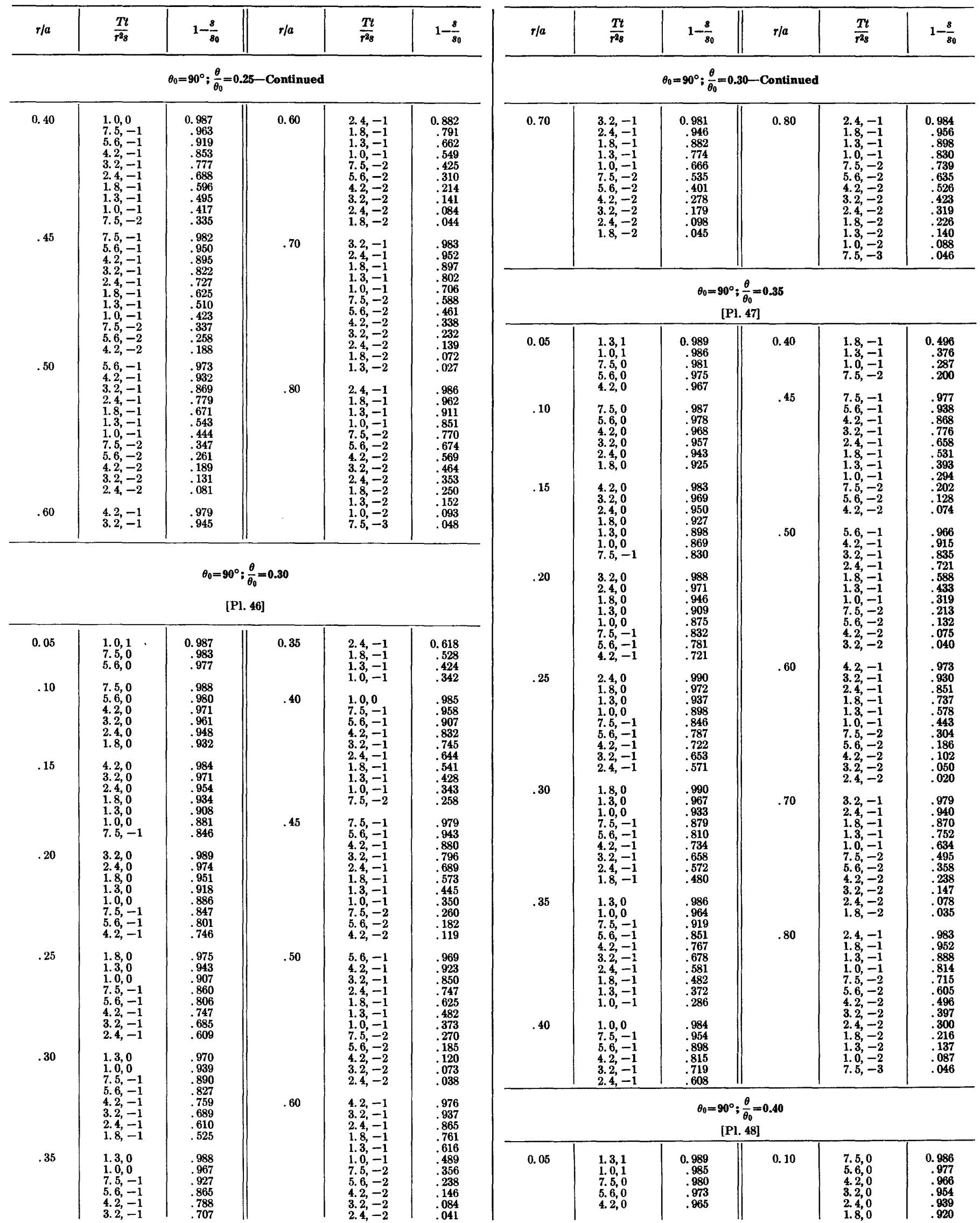




\begin{tabular}{|c|c|c|c|c|c|}
\hline$r / a$ & $\frac{T t}{r^{2} 8}$ & $1-\frac{8}{80}$ & $r / a$ & $\frac{T t}{r^{28}}$ & $1-\frac{8}{80}$ \\
\hline \multicolumn{6}{|c|}{$\theta_{0}=90^{\circ} ; \frac{\theta}{\theta_{0}}=0.40-$ Continued } \\
\hline 0.15 & $\begin{array}{l}4.2,0 \\
3.2,0 \\
2.4,0 \\
1.8,0 \\
1.3,0 \\
1.0,0 \\
7.5,-1\end{array}$ & $\begin{array}{r}0.981 \\
.966 \\
.946 \\
.923 \\
.891 \\
.861 \\
.819\end{array}$ & \multirow[t]{2}{*}{0.45} & \multirow{2}{*}{$\begin{array}{l}7.5,-1 \\
5.6,-1 \\
4.2,-1 \\
\text {.2, } 2,1 \\
2.4,-1 \\
1.8,-1 \\
1.3,-1 \\
1.0,-1 \\
7.5,-2 \\
\text { 4. } 6,-2 \\
\text { 4. } 2,-2\end{array}$} & \multirow{2}{*}{$\begin{array}{r}0.976 \\
.933 \\
.859 \\
.761 \\
.635 \\
.501 \\
.357 \\
.255 \\
.163 \\
.094 \\
.048\end{array}$} \\
\hline .20 & $\begin{array}{l}3.2,0 \\
2.4,0 \\
1.8,0\end{array}$ & $\begin{array}{l}.987 \\
.969 \\
.943\end{array}$ & & & \\
\hline & $\begin{array}{l}1.3,0 \\
1.0,0 \\
7.5,-1 \\
5.6,-1 \\
4.2,-1\end{array}$ & $\begin{array}{l}.903 \\
.866 \\
.821 \\
.766 \\
.702\end{array}$ & \multirow[t]{2}{*}{.50} & \multirow{2}{*}{$\begin{array}{l}\text { 7.5, } \\
\text { 5. } 6,-1 \\
\text { 4. } 2,-1 \\
\text { 3. } 2,-1 \\
\text { 2.4, -1 } \\
1.8,-1 \\
1.3,-1 \\
\text { 1.0, -1 } \\
\text { 5.5, -2 } \\
\text { 4. } 2,-2\end{array}$} & \multirow{2}{*}{$\begin{array}{l}.990 \\
.964 \\
.909 \\
.824 \\
.703 \\
.561 \\
.399 \\
.280 \\
.175 \\
.097 \\
.048\end{array}$} \\
\hline .25 & $\begin{array}{l}2.4,0 \\
1.8,0 \\
1.3,0 \\
1.0,0 \\
7.5,-1 \\
5,6,-1\end{array}$ & $\begin{array}{l}.989 \\
.971 \\
.933 \\
.891 \\
.835 \\
.772\end{array}$ & & & \\
\hline & $\begin{array}{l}0.0,-1 \\
4.2,-1 \\
3.2,-1 \\
2.4,-1\end{array}$ & $\begin{array}{l}.704 \\
.630 \\
.543\end{array}$ & \multirow[t]{2}{*}{.60} & \multirow{2}{*}{$\begin{array}{l}\text { 4. } 2,-1 \\
\text { 3. } 2,-1 \\
2.4,-1 \\
1.8,-1 \\
\text { 1. } 3,-1 \\
\text { 1. } 0,-1 \\
\text { 7. } 5,-2 \\
\text { 4. } 6,-2 \\
\text { 3. } 2,-2\end{array}$} & \multirow{2}{*}{$\begin{array}{l}.971 \\
.926 \\
.841 \\
.720 \\
.552 \\
.410 \\
.268 \\
.153 \\
.075 \\
.032\end{array}$} \\
\hline .30 & $\begin{array}{l}1.8,0 \\
1.3,0 \\
1.0,0 \\
7.5,-1 \\
5.6,-1 \\
4.2,-1\end{array}$ & $\begin{array}{l}.989 \\
.965 \\
.928 \\
.870 \\
. .797 \\
.717\end{array}$ & & & \\
\hline & $\begin{array}{l}\text { 4. } 2,-1 \\
3 .,-1 \\
2.4,-1 \\
1.8,-1\end{array}$ & $\begin{array}{l}.635 \\
.544 \\
.447\end{array}$ & \multirow[t]{2}{*}{.70} & \multirow{2}{*}{$\begin{array}{l}3.2,-1 \\
2.4,-1 \\
1.8,-1 \\
1.3,-1 \\
1.0,-1 \\
7.5,-2 \\
5.6,-2 \\
4.2,-2 \\
3.2,-2 \\
2.4,-2 \\
1.8,-2\end{array}$} & \multirow{2}{*}{$\begin{array}{l}.977 \\
.936 \\
. .861 \\
.735 \\
.611 \\
.467 \\
.328 \\
.213 \\
.130 \\
.068 \\
.031\end{array}$} \\
\hline .35 & $\begin{array}{l}1.3,0 \\
1.0,0 \\
7.5,-1 \\
5.6,-1 \\
4.2,-1 \\
3.2,-1 \\
2.4,-1 \\
1.8,-1\end{array}$ & $\begin{array}{l}.985 \\
.961 \\
.914 \\
.841 \\
.751 \\
.657 \\
.554 \\
.450\end{array}$ & & & \\
\hline & $\begin{array}{l}\text { 1. } 3,-1 \\
\text { 1. } 0,-1\end{array}$ & $\begin{array}{l}.334 \\
.246\end{array}$ & \multirow[t]{2}{*}{.80} & \multirow{2}{*}{$\begin{array}{l}2.4,-1 \\
1.8,-1 \\
1.3,-1 \\
1.0,-1 \\
7.5,-2 \\
5.6,-2 \\
4.2,-2 \\
3.2,-2 \\
2.4,-2 \\
1.8,-2 \\
1.3,-2 \\
1.0,-2 \\
7.5,-3\end{array}$} & \multirow{2}{*}{$\begin{array}{l}.981 \\
.949 \\
.880 \\
.802 \\
.698 \\
.585 \\
.477 \\
.382 \\
.292 \\
.212 \\
.136 \\
.086 \\
.046\end{array}$} \\
\hline .40 & $\begin{array}{l}1.0,0 \\
7.5,-1 \\
5.6,-1 \\
4.2,-1 \\
3.2,-1 \\
2.4,-1 \\
1.8,-1 \\
1.3,-1 \\
1.0,-1 \\
7.5,-2\end{array}$ & $\begin{array}{l}.983 \\
.9851 \\
.891 \\
.803 \\
.701 \\
.583 \\
.464 \\
.339 \\
.247 \\
.161\end{array}$ & & & \\
\hline
\end{tabular}

$\theta_{0}=90^{\circ} ; \frac{\theta}{\theta_{0}}=0.45$

[Pl. 49]

\begin{tabular}{|c|c|c|c|c|c|}
\hline 0.05 & $\begin{array}{l}1.3,1 \\
1.0,1 \\
7.5,0 \\
5.6,0 \\
4.2,0\end{array}$ & $\begin{array}{r}0.988 \\
.984 \\
.979 \\
.972 \\
.963\end{array}$ & \multirow[t]{2}{*}{0.25} & \multirow{2}{*}{$\begin{array}{l}2.4,0 \\
1.8,0 \\
1.3,0 \\
1.0,0 \\
7.5,-1 \\
\text { 5. } 6,-1 \\
\text { 4. } 2,-1 \\
\text { 3. } 2,-1 \\
2 .,-1\end{array}$} & $\begin{array}{r}0.989 \\
.969 \\
.931 \\
.887 \\
.829\end{array}$ \\
\hline .10 & $\begin{array}{l}7.5,0 \\
5.6,0 \\
4.2,0\end{array}$ & $\begin{array}{r}.985 \\
.976 \\
.965\end{array}$ & & & $\begin{array}{l}.692 \\
.6917 \\
.562\end{array}$ \\
\hline & $\begin{array}{l}2.2,0 \\
2.4,0 \\
1.8,0 \\
1.3,0\end{array}$ & $\begin{array}{r}.953 \\
.937 \\
.917 \\
.886\end{array}$ & \multirow[t]{2}{*}{.30} & \multirow{2}{*}{$\begin{array}{l}1.8,0 \\
1.3,0 \\
1.0,0 \\
7.5,-1 \\
\text { S. } 6,-1 \\
\text { 4. } 2,-1 \\
\text { 3. } 2,-1 \\
\text { 2. 4, - } \\
\text { 1.8, - }\end{array}$} & $\begin{array}{r}.989 \\
.963 \\
.925\end{array}$ \\
\hline .15 & $\begin{array}{l}4.2,0 \\
3.2,0 \\
2.4,0 \\
1.8,0 \\
1.3,0\end{array}$ & $\begin{array}{l}.981 \\
.965 \\
.944 \\
.920 \\
.887\end{array}$ & & & $\begin{array}{l}.780 \\
.789 \\
.622 \\
.527 \\
.427\end{array}$ \\
\hline & $\begin{array}{l}1.0,0 \\
7.5,-1\end{array}$ & .855 & \multirow[t]{2}{*}{.35} & \multirow[b]{2}{*}{$\begin{array}{l}1.3,0 \\
1.0,0 \\
7.5,-1 \\
5.6,-1 \\
4.2,-1 \\
3.2,-1 \\
2.4,-1 \\
1.8,-1 \\
1.3,-1 \\
1.0,-1\end{array}$} & .985 \\
\hline .20 & $\begin{array}{l}3.2,0 \\
2.4,0 \\
1.8,0 \\
1.3,0 \\
1.0,0 \\
7.5,-1 \\
5.6,-1 \\
4.2,-1\end{array}$ & $\begin{array}{l}.986 \\
.968 \\
.940 \\
.899 \\
.861 \\
.814 \\
.757 \\
.691\end{array}$ & & & $\begin{array}{l}.910 \\
.835 \\
.742 \\
.644 \\
.537 \\
.430 \\
.312 \\
.223\end{array}$ \\
\hline
\end{tabular}

\begin{tabular}{|c|c|c|c|c|c|}
\hline$r / a$ & $\frac{T t}{r^{2} s}$ & $1-\frac{8}{80}$ & $r / a$ & $\frac{T t}{r^{23}}$ & $1-\frac{8}{80}$ \\
\hline \multicolumn{6}{|c|}{$\theta_{0}=90^{\circ} ; \frac{\theta}{\theta_{0}}=0.45-$ Continued } \\
\hline 0.40 & $\begin{array}{l}1.0,0 \\
7.5,-1 \\
5.6,-1 \\
4.2,-1 \\
3.2,-1 \\
2.4,-1 \\
1.8,-1 \\
1.3,-1 \\
1.0,-1 \\
7.5,-2\end{array}$ & $\begin{array}{r}0.982 \\
.949 \\
.886 \\
.795 \\
.689 \\
.567 \\
.445 \\
.316 \\
.224 \\
.139\end{array}$ & 0.60 & $\begin{array}{l}4.2,-1 \\
3.2,-1 \\
2.4,-1 \\
1.8,-1 \\
1.3,-1 \\
1.0,-1 \\
7.5,-2 \\
5.6,-2 \\
4.2,-2 \\
3.2,-2\end{array}$ & $\begin{array}{r}0.970 \\
.923 \\
.835 \\
.709 \\
.535 \\
.390 \\
.247 \\
.134 \\
.061 \\
.024\end{array}$ \\
\hline .45 & $\begin{array}{l}7.5,-1 \\
5.6,-1 \\
4.2,-1 \\
3.2,-1 \\
2.4,-1 \\
1.8,-1 \\
1.3,-1 \\
1.0,-1 \\
7.5,-2 \\
5.6,-2 \\
4.2,-2\end{array}$ & $\begin{array}{l}.975 \\
.931 \\
.854 \\
.752 \\
.621 \\
.483 \\
.335 \\
.232 \\
.141 \\
.075 \\
.034\end{array}$ & .70 & $\begin{array}{l}3.2,-1 \\
2.4,-1 \\
1.8,-1 \\
1.3,-1 \\
1.0,-1 \\
7.5,-2 \\
5.6,-2 \\
4.2,-2 \\
3.2,-2 \\
.4,-2 \\
1.8,-2\end{array}$ & $\begin{array}{l}.976 \\
.934 \\
.856 \\
.775 \\
.597 \\
.450 \\
. .212 \\
.200 \\
.121 \\
.004 \\
.029\end{array}$ \\
\hline .50 & $\begin{array}{l}7.5,-1 \\
5.6,-1 \\
4.2,-1 \\
3.2,-1 \\
2.4,-1 \\
1.8,-1 \\
1.3,-1 \\
1.0,-1 \\
7.5,-2 \\
5.6,-2 \\
4.2,-2\end{array}$ & $\begin{array}{l}.989 \\
.962 \\
.905 \\
.817 \\
.692 \\
.545 \\
.378 \\
.258 \\
.152 \\
.078 \\
.034\end{array}$ & .80 & $\begin{array}{l}2.4,-1 \\
1.8,-1 \\
1.3,-1 \\
1.0,-1 \\
7.5,-2 \\
5.6,-2 \\
4.2,-2 \\
3.2,-2 \\
2.4,-2 \\
1.8,-2 \\
1.3,-2 \\
1.0,-2 \\
7.5,-3\end{array}$ & $\begin{array}{l}.981 \\
.947 \\
.876 \\
.794 \\
.688 \\
.573 \\
.466 \\
.375 \\
.288 \\
.211 \\
.136 \\
.086 \\
.046\end{array}$ \\
\hline
\end{tabular}

[P1. 50]

\begin{tabular}{|c|c|c|c|c|c|}
\hline 0.05 & $\begin{array}{l}1.3,1 \\
1.0,1 \\
7.5,0 \\
5.6,0 \\
4.2,0\end{array}$ & $\begin{array}{r}0.988 \\
.984 \\
.979 \\
.972 \\
.963\end{array}$ & 0.35 & $\begin{array}{l}7.5,-1 \\
5.6,-1 \\
4.2,-1 \\
3.2,-1 \\
2.4,-1 \\
1.8,-1\end{array}$ & $\begin{array}{r}0.909 \\
.833 \\
.738 \\
.639 \\
.532 \\
.423\end{array}$ \\
\hline \multirow[t]{2}{*}{.10} & $\begin{array}{l}7.5,0 \\
5.6,0\end{array}$ & $\begin{array}{l}.985 \\
.975\end{array}$ & \multirow{3}{*}{.40} & $\begin{array}{l}1.3,-1 \\
1.0,-1\end{array}$ & $\begin{array}{l}.304 \\
.215\end{array}$ \\
\hline & $\begin{array}{l}4.2,0 \\
3.2,0 \\
2.4,0 \\
1.8,0 \\
1.3,0\end{array}$ & $\begin{array}{l}.964 \\
.952 \\
.936 \\
.916 \\
.885\end{array}$ & & $\begin{array}{l}1.0,0 \\
7.5,-1 \\
\text { 5. } 6,-1 \\
\text { 4. } 2,-1 \\
\text { 3. } 2,-1\end{array}$ & $\begin{array}{l}.982 \\
.948 \\
.885 \\
.793 \\
.686\end{array}$ \\
\hline \multirow[t]{2}{*}{.15} & $\begin{array}{l}4.2,0 \\
3.2,0 \\
2.4,0 \\
1.8,0\end{array}$ & $\begin{array}{l}.980 \\
.965 \\
.943 \\
.919\end{array}$ & & $\begin{array}{l}2.4,-1 \\
1.8,-1 \\
1.3,-1 \\
1.0,-1 \\
7.5,-2\end{array}$ & $\begin{array}{l}.562 \\
.439 \\
.309 \\
.216 \\
.131\end{array}$ \\
\hline & $\begin{array}{l}1.3,0 \\
7.0,0 \\
7.5,-1\end{array}$ & $\begin{array}{l}.886 \\
.853 \\
.810\end{array}$ & \multirow[t]{2}{*}{.45} & $\begin{array}{l}7.5,-1 \\
5.6,=1\end{array}$ & $\begin{array}{l}.975 \\
.930 \\
.852\end{array}$ \\
\hline .20 & $\begin{array}{l}3.2,0 \\
2.4,0 \\
1.8,0 \\
1.3,0 \\
1.0,0 \\
7.5,-1 \\
5.6,-1 \\
4.2,-1\end{array}$ & $\begin{array}{l}.986 \\
.968 \\
.940 \\
.898 \\
.859 \\
.811 \\
.754 \\
.687\end{array}$ & & $\begin{array}{l}\text { 3. } 2,-1 \\
2.4,-1 \\
1.8,-1 \\
1.3,-1 \\
1.0,-1 \\
7.5,-2 \\
5.6,-2 \\
4.2,-2\end{array}$ & $\begin{array}{l}.748 \\
.617 \\
.477 \\
.327 \\
.224 \\
.134 \\
.068 \\
.029\end{array}$ \\
\hline .25 & $\begin{array}{l}2.4,0 \\
1.8,0 \\
1.3,0 \\
1.0,0 \\
7.5,-1 \\
5,6,-1 \\
4.2,-1 \\
3.2,-1 \\
2.4,-1\end{array}$ & $\begin{array}{l}.988 \\
.969 \\
.930 \\
.885 \\
.827 \\
.761 \\
.689 \\
.612 \\
.520\end{array}$ & .50 & $\begin{array}{l}7.5,-1 \\
5.6,-1 \\
4.2,-1 \\
3.2,-1 \\
2.4,-1 \\
1.8,-1 \\
1.3,-1 \\
1.0,-1 \\
7.5,-2 \\
5.6,-2\end{array}$ & $\begin{array}{l}.989 \\
.962 \\
.904 \\
.815 \\
.688 \\
.540 \\
.371 \\
.250 \\
. .145 \\
.072\end{array}$ \\
\hline \multirow[t]{2}{*}{.30} & $\begin{array}{l}1.8,0 \\
1.3 .0\end{array}$ & $\begin{array}{l}.989 \\
.963\end{array}$ & & 4. $2,-2$ & .030 \\
\hline & $\begin{array}{l}1.5,0 \\
1.0,0 \\
7.5,-1 \\
5.6,-1 \\
4.2,-1 \\
3.2,-1 \\
.4,-1 \\
1.8,-1\end{array}$ & $\begin{array}{l}.805 \\
.924 \\
.867 \\
.702 \\
.617 \\
.522 \\
.420\end{array}$ & \multirow[t]{2}{*}{.60} & $\begin{array}{l}\text { 4. } 2,-1 \\
3.2,-1 \\
2.4,-1 \\
1.8,-1 \\
1.3,-1 \\
1.0,-1 \\
7.5,-2 \\
5.6,-2\end{array}$ & $\begin{array}{l}.970 \\
.922 \\
.833 \\
.705 \\
.530 \\
.384 \\
.240 \\
128\end{array}$ \\
\hline .35 & $\begin{array}{l}1.3,0 \\
1.0,0\end{array}$ & $\begin{array}{l}.985 \\
.960\end{array}$ & & $\begin{array}{l}4.2,-2 \\
3.2,-2\end{array}$ & .057 \\
\hline
\end{tabular}




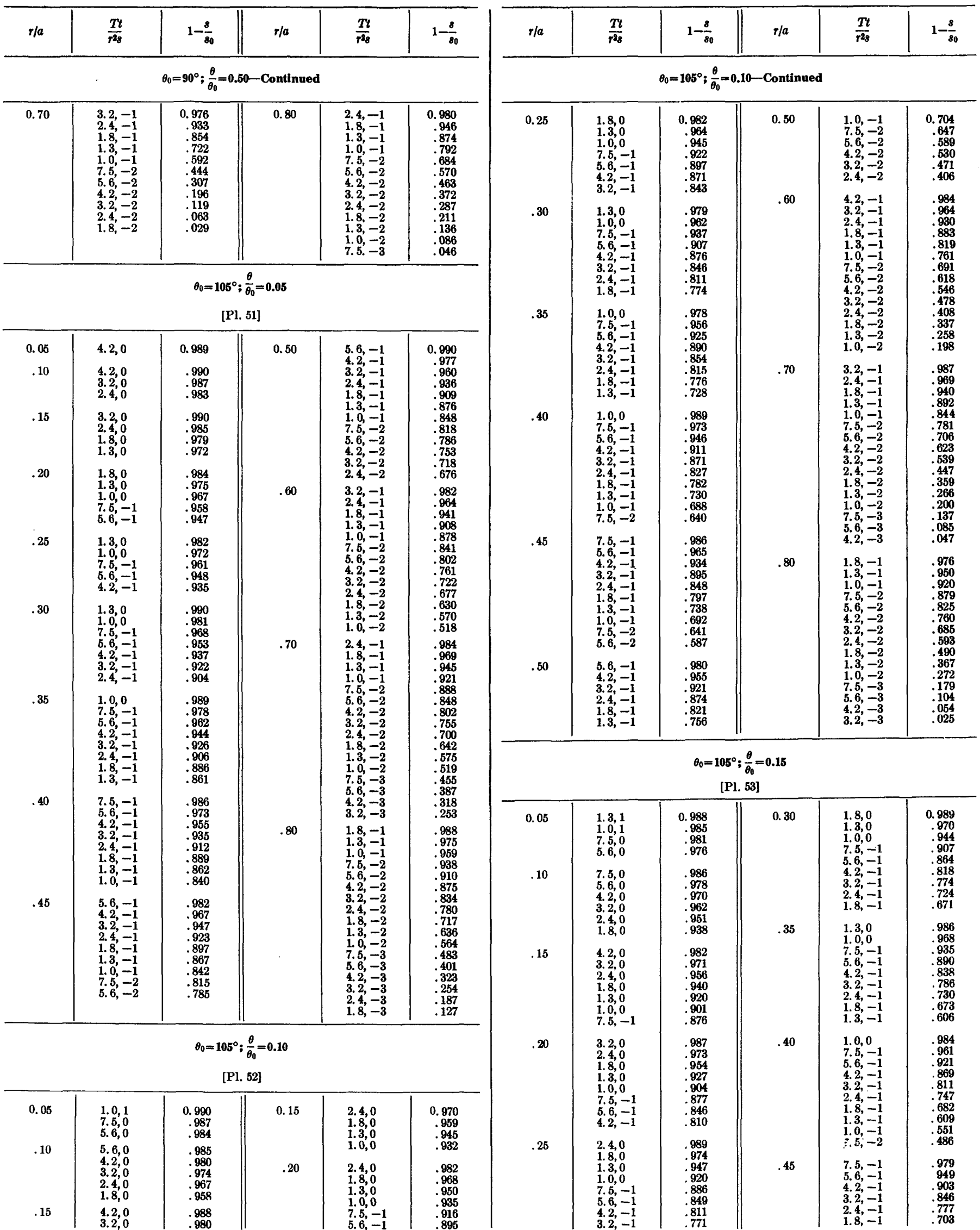




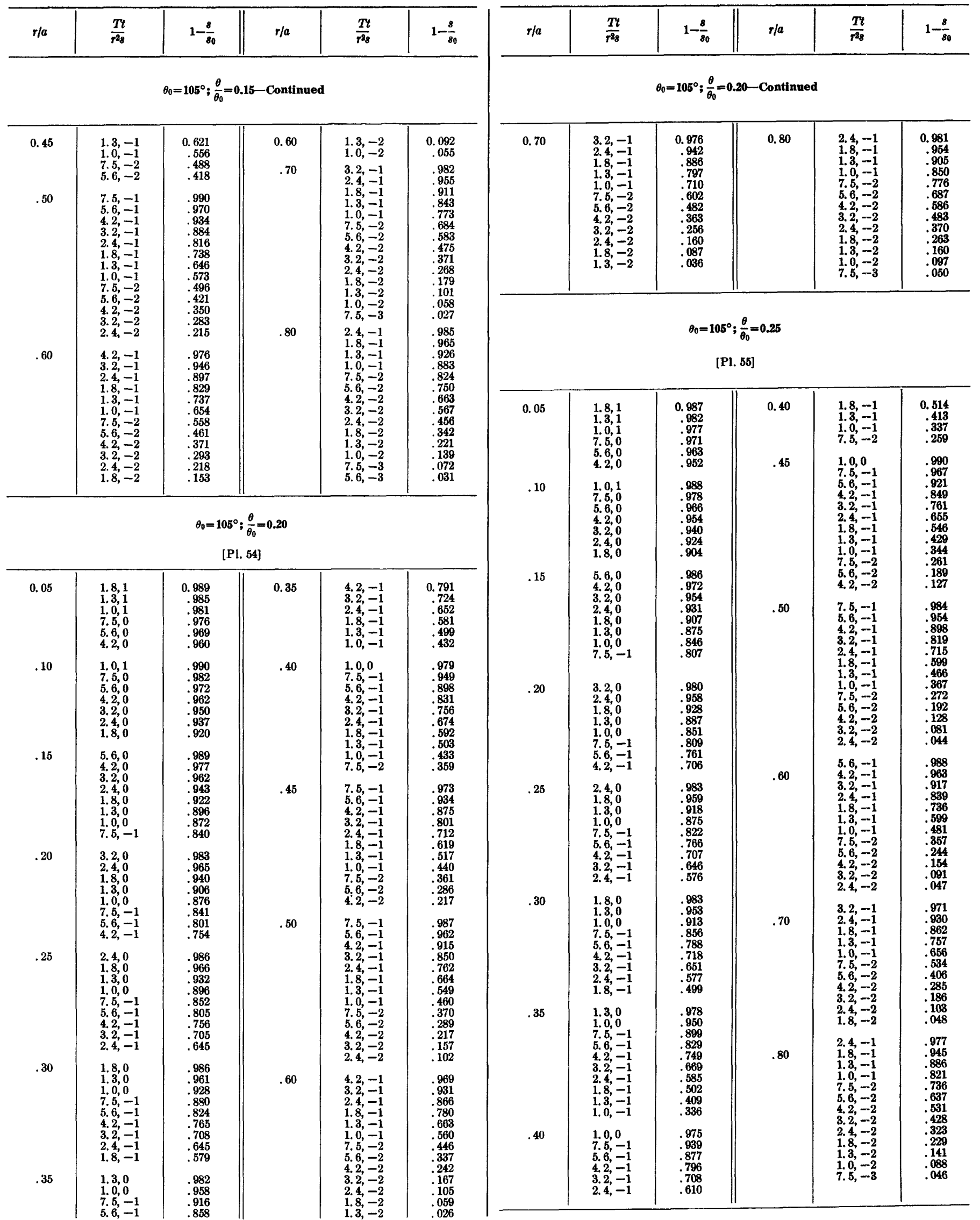

791-598 O-66-4 
HYDRAULIC DIFFUSIVITY, WEDGE-SHAPED AQUIFERS DRAINED BY STREAMS

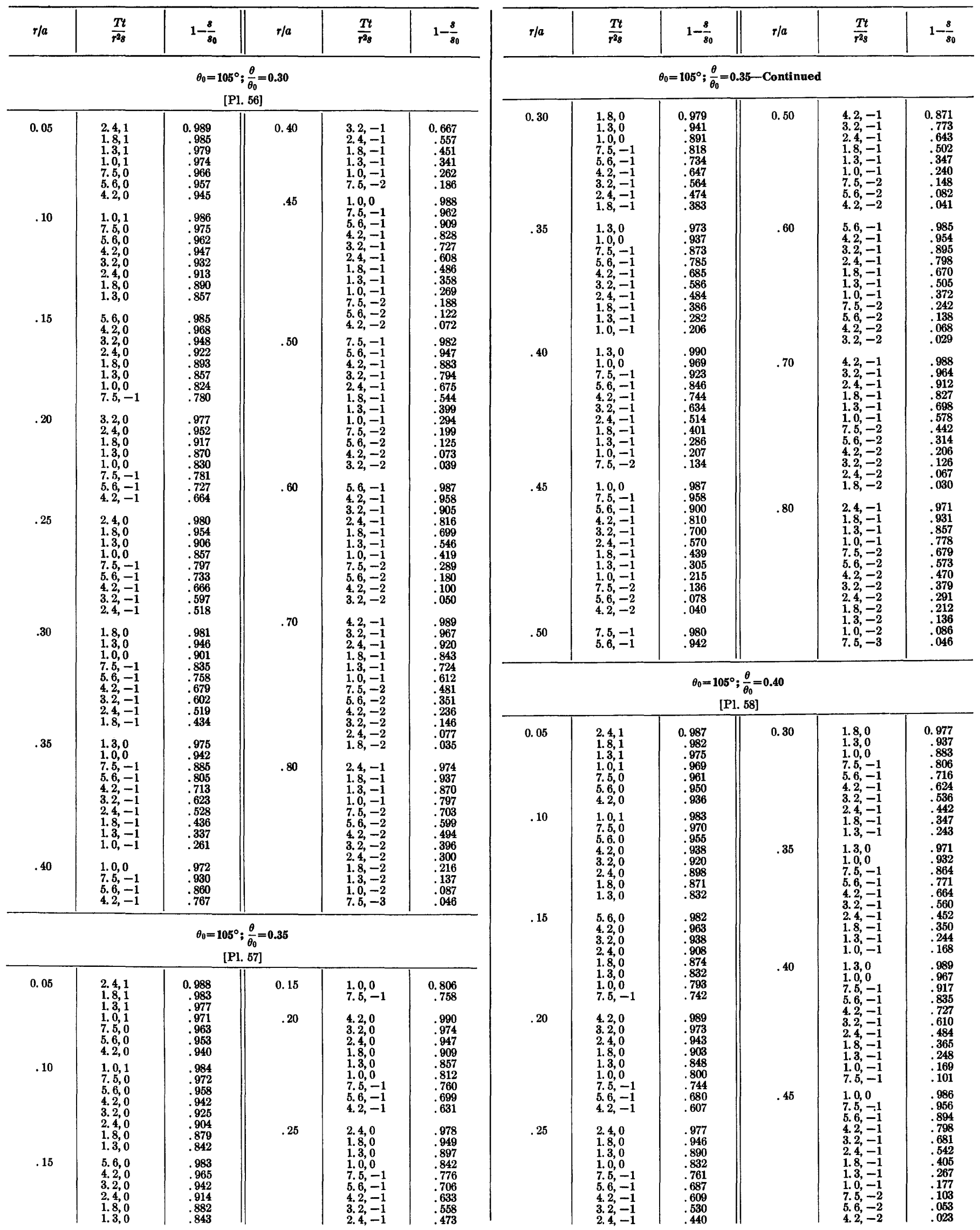




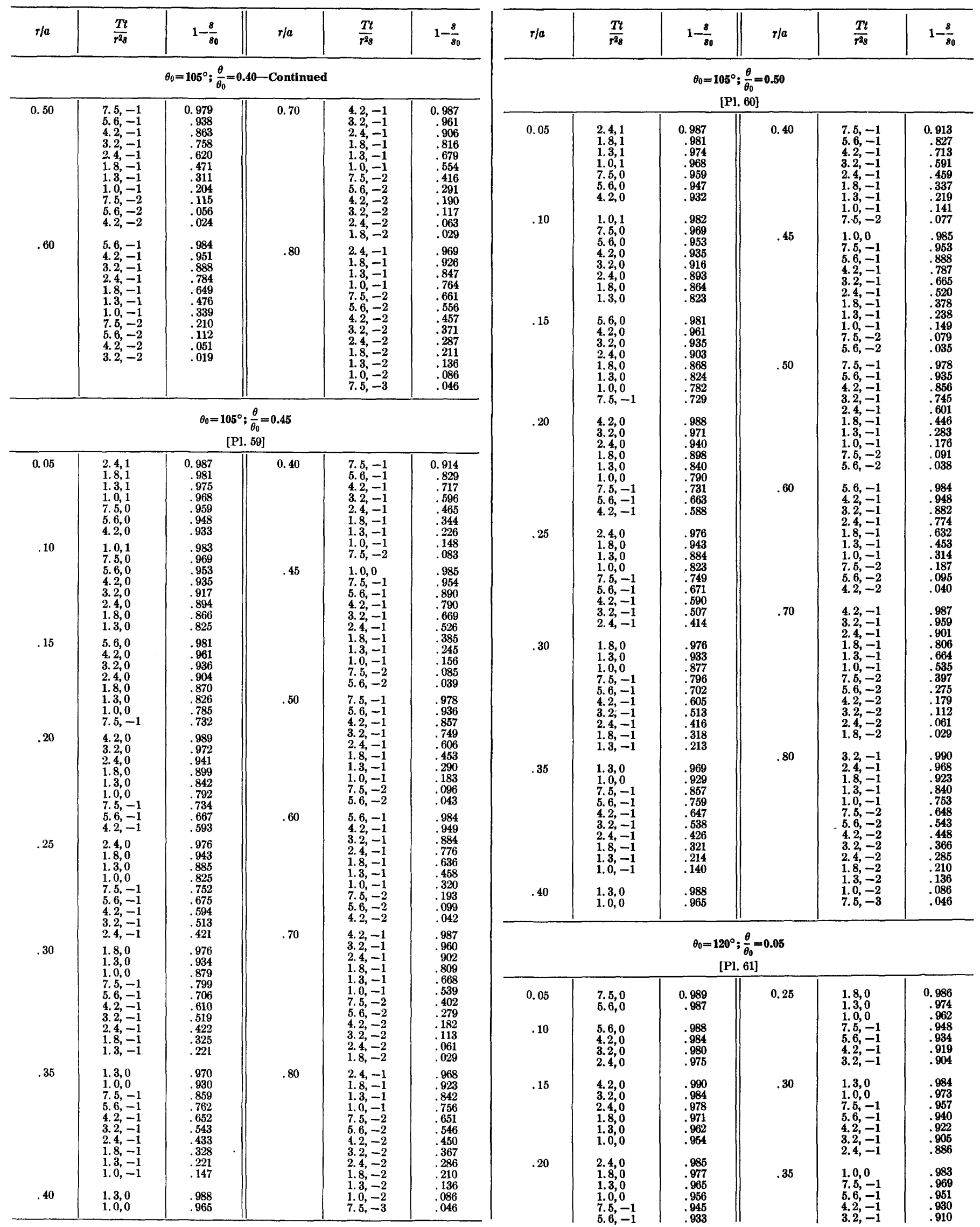




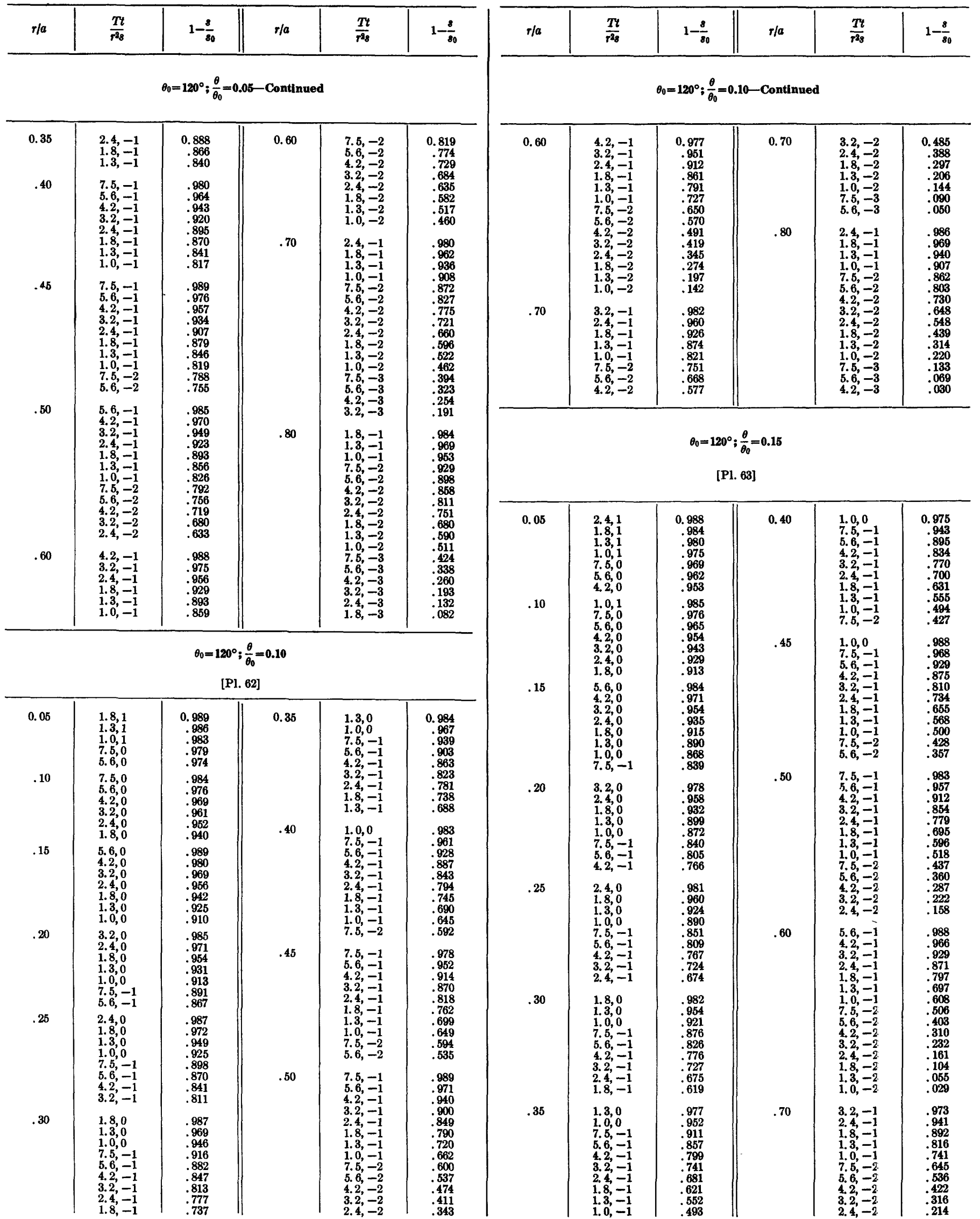




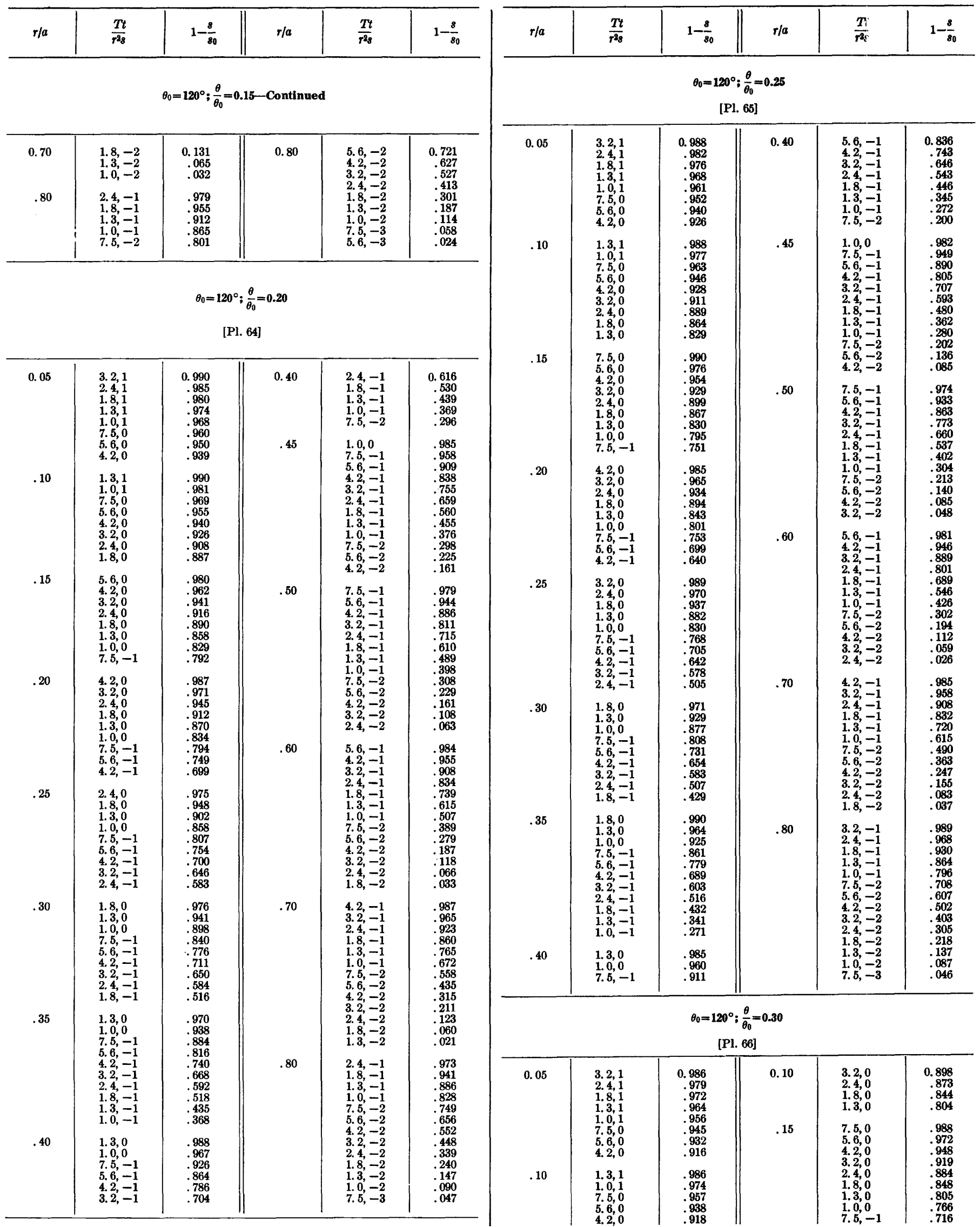


HYDRAULIC DIFFUSIVITY, WEDGE-SHAPED AQUIFERS DRAINED BY STREAMS

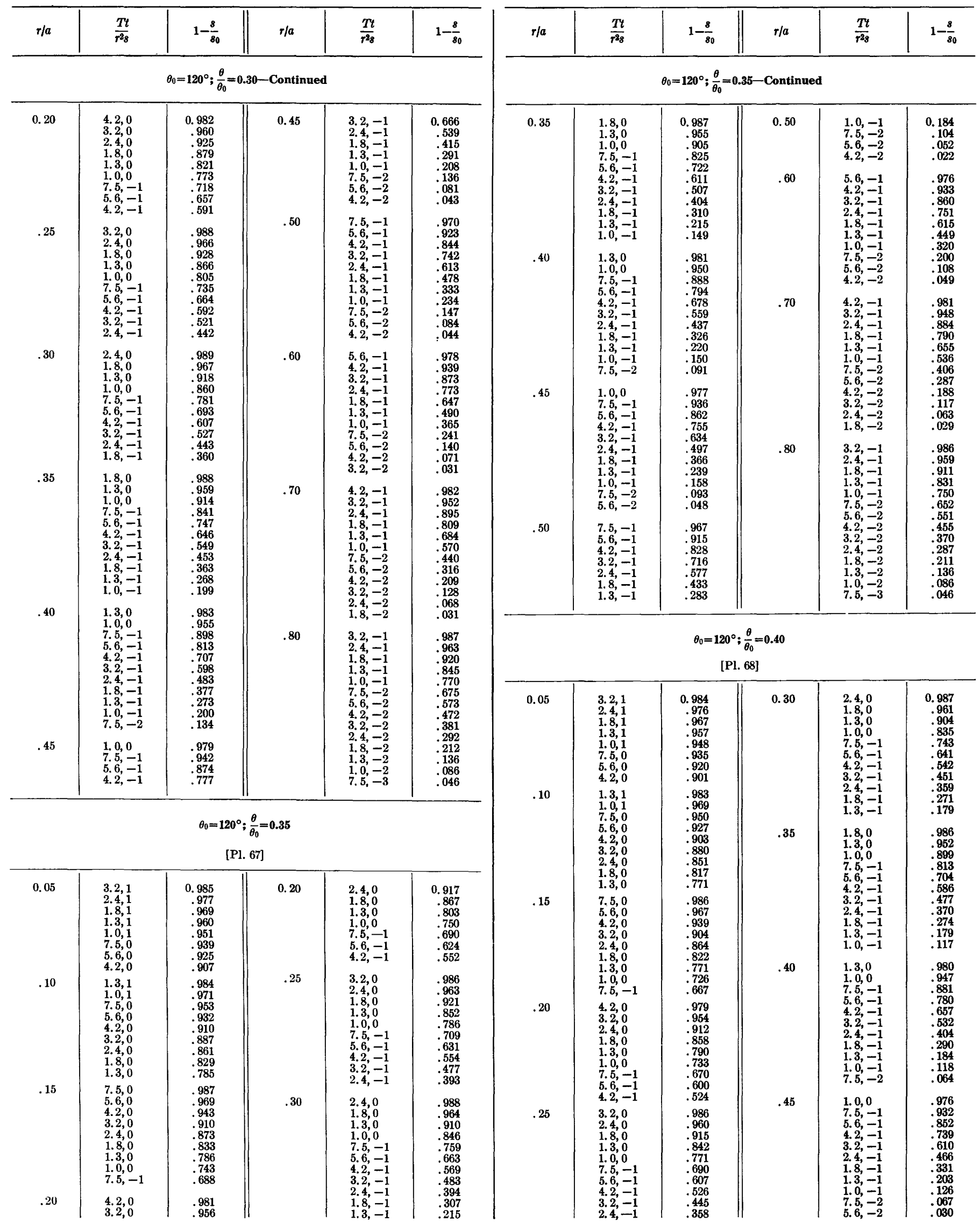


TABLES

\begin{tabular}{c|c|c||c|c|c}
\hline$r / a$ & $\frac{T t}{r^{2}}$ & $1-\frac{s}{s_{0}}$ & $r / a$ & $\frac{T t}{r^{2} s}$ & $1-\frac{s}{s_{0}}$ \\
$\theta_{0}=120^{\circ} ; \frac{\theta}{\theta_{0}}=0.40-$ Continued
\end{tabular}

\begin{tabular}{|c|c|c|c|c|c|}
\hline 0.50 & $\begin{array}{l}7.5,-1 \\
5.6,-1 \\
4.2,-1 \\
3.2,-1 \\
2.4,-1 \\
1.8,-1 \\
1.3,-1 \\
1.0,-1 \\
7.5,-2 \\
5.6,-2\end{array}$ & $\begin{array}{r}0.965 \\
.909 \\
.817 \\
.698 \\
.551 \\
.400 \\
.249 \\
.152 \\
.078 \\
.034\end{array}$ & 0.70 & $\begin{array}{l}\text { 1. } 8,-1 \\
\text { 1. } 3,-1 \\
\text { 1. } 0,-1 \\
\text { 7. } 5,-2 \\
5.6,-2 \\
4.2,-2 \\
\text { 3. } 2,-2 \\
2.4,-2 \\
1.8,-2\end{array}$ & $\begin{array}{r}0.777 \\
.635 \\
.513 \\
.384 \\
.270 \\
.178 \\
.112 \\
.061 \\
.029\end{array}$ \\
\hline .60 & $\begin{array}{l}\text { 5. } 6,-1 \\
4.2,-1 \\
3.2,-1 \\
2.4,-1 \\
1.8,-1 \\
1.3,-1 \\
1.0,-1 \\
7.5,-2 \\
5 .,-2 \\
4.2,-2\end{array}$ & $\begin{array}{l}.974 \\
.928 \\
.851 \\
.734 \\
.591 \\
.420 \\
.291 \\
.175 \\
.090 \\
.039\end{array}$ & .80 & $\begin{array}{l}\text { 3. } 2,-1 \\
2.4,-1 \\
1.8,-1 \\
1.3,-1 \\
1.0,-1 \\
7.5,-2 \\
5.6,-2 \\
4.2,-2 \\
3.2,-2 \\
2.4,-2\end{array}$ & $\begin{array}{l}.985 \\
.957 \\
.906 \\
.820 \\
.766 \\
.637 \\
.538 \\
.447 \\
. .366 \\
.285\end{array}$ \\
\hline .70 & $\begin{array}{l}\text { 4. } 2,-1 \\
3.2,-1 \\
2.4,-1\end{array}$ & $\begin{array}{l}.979 \\
.944 \\
.877\end{array}$ & & $\begin{array}{l}1.3,-2 \\
1.0,-2 \\
7.5,-3\end{array}$ & $\begin{array}{l}.136 \\
.086 \\
.046\end{array}$ \\
\hline
\end{tabular}

\begin{tabular}{|c|c|c|c|c|c|}
\hline 0.05 & $\begin{array}{l}4.2,1 \\
3.2,1 \\
2.4,1 \\
1.8,1 \\
1.3,1 \\
1.0,1 \\
7.5,0 \\
5.6,0 \\
4.2,0\end{array}$ & $\begin{array}{r}0.990 \\
.983 \\
.975 \\
.966 \\
.956 \\
.946 \\
.933 \\
.917 \\
.897\end{array}$ & 0.35 & $\begin{array}{l}1.0,0 \\
7.5,-1 \\
5.6,-1 \\
4.2,-1 \\
3.2,-1 \\
2.4,-1 \\
1.8,-1 \\
1.3,-1 \\
1.0,-1\end{array}$ & $\begin{array}{r}0.895 \\
.806 \\
.693 \\
. .471 \\
.459 \\
.349 \\
.252 \\
.159 \\
.099\end{array}$ \\
\hline .10 & $\begin{array}{l}1.3,1 \\
1.0,1 \\
7.5,0 \\
5.6,0 \\
4.2,0 \\
3.2,0 \\
2.4,0 \\
1.8,0 \\
1.3,0\end{array}$ & $\begin{array}{l}.983 \\
.968 \\
.948 \\
.924 \\
.900 \\
.875 \\
.846 \\
.810 \\
.762\end{array}$ & .40 & $\begin{array}{l}1.3,0 \\
1.0,0 \\
7.5,-1 \\
5.6,-1 \\
\text { 4. } 2,-1 \\
\text { 3.2, } \\
\text { 2. } 4,-1 \\
1.8,-1 \\
\text { 1. } 3,-1\end{array}$ & $\begin{array}{r}.979 \\
.945 \\
. .776 \\
.772 \\
.644 \\
. .384 \\
. .269 \\
. .64\end{array}$ \\
\hline .15 & $\begin{array}{l}7.5,0 \\
5,6,0\end{array}$ & $\begin{array}{r}.986 \\
.966\end{array}$ & & $7.5,-2$ & 051 \\
\hline & $\begin{array}{l}4 ., 2,0 \\
4.2,0 \\
3.2,0 \\
1.8,0 \\
1.8,0 \\
1.0,0 \\
7.5,-1\end{array}$ & $\begin{array}{l}.900 \\
.936 \\
.901 \\
.859 \\
.763 \\
.715 \\
.655\end{array}$ & .45 & $\begin{array}{l}\text { 1. } 0,0 \\
\text { 7. } 5,-1 \\
\text { 5. } 6,-1 \\
\text { 4. } 2,-1 \\
\text { 3. } 2,-1 \\
2.4,-1 \\
\text { 1. } 8,-1\end{array}$ & $\begin{array}{l}.975 \\
.929 \\
.847 \\
.729 \\
.596 \\
.448 \\
.310 \\
.922\end{array}$ \\
\hline .20 & $\begin{array}{l}4.2,0 \\
3.2,0 \\
2.4,0 \\
1.8,0\end{array}$ & $\begin{array}{l}.978 \\
.952 \\
.908 \\
.852\end{array}$ & & $\begin{array}{l}1.0,-1 \\
7.5,-2 \\
5.6,-2\end{array}$ & $\begin{array}{r}.108 \\
.053 \\
.021\end{array}$ \\
\hline & $\begin{array}{l}1.3,0 \\
1.0,0 \\
7.5,-1 \\
5 .,-1 \\
4.2,-1\end{array}$ & $\begin{array}{l}.782 \\
.723 \\
.657 \\
.585 \\
.507\end{array}$ & .50 & $\begin{array}{l}1.0,0 \\
7.5,-1 \\
\text { 5. } 6,-1 \\
\text { 4. } 2,-1 \\
\text { 3. } 2,-1\end{array}$ & $\begin{array}{l}.990 \\
.964 \\
.906 \\
.810 \\
.686\end{array}$ \\
\hline .25 & $\begin{array}{l}3.2,0 \\
2,4,0 \\
1.8,0 \\
1.3,0 \\
1.0,0\end{array}$ & $\begin{array}{l}.985 \\
.959 \\
.912 \\
.836 \\
.763\end{array}$ & & $\begin{array}{l}\text { 1.8, } \\
1.3,-1 \\
\text { 1. } 0,-1 \\
\text { 7. } 5,-2 \\
\text {. } 6,-2\end{array}$ & $\begin{array}{l}.0281 \\
.229 \\
.225 \\
.064 \\
.025\end{array}$ \\
\hline & $\begin{array}{l}7.6,-1 \\
5 .,-1 \\
4 .,-1 \\
3.2,-1 \\
2.4,-1\end{array}$ & $\begin{array}{l}.678 \\
.593 \\
.509 \\
.426 \\
.337\end{array}$ & .60 & $\begin{array}{l}5.6,-1 \\
4.2,-1 \\
3.2,-1 \\
2.4,-1\end{array}$ & $\begin{array}{l}.973 \\
.925 \\
.845 \\
.725\end{array}$ \\
\hline .30 & $\begin{array}{l}2.4,0 \\
1.8,0 \\
1.3,0 \\
1.00 \\
7.5,-1\end{array}$ & $\begin{array}{l}.986 \\
.960 \\
.900 \\
.829 \\
.733 \\
.738\end{array}$ & & $\begin{array}{l}\text { 1. } 3,-1 \\
\text { 1. } 0,-1 \\
\text { 5. } 5,-2 \\
\text { 4. } 2,-2\end{array}$ & $\begin{array}{l}.403 \\
.275 \\
.161 \\
.081 \\
.034\end{array}$ \\
\hline & $\begin{array}{l}5.6,-1 \\
4.2,-1 \\
3.2,-1 \\
2.4,-1 \\
1.8,-1 \\
1.3,-1\end{array}$ & $\begin{array}{r}.628 \\
.525 \\
.432 \\
.338 \\
.249 \\
.158\end{array}$ & .70 & $\begin{array}{l}\text { 4. } 2,-1 \\
\text { 3. } 2,-1 \\
2.4,-1 \\
\text { 1. } 8,-1 \\
\text { 1. } 3,-1\end{array}$ & $\begin{array}{l}.978 \\
.942 \\
.872 \\
.769 \\
.624\end{array}$ \\
\hline .35 & $\begin{array}{l}1.8,0 \\
1.3,0\end{array}$ & $\begin{array}{l}.985 \\
.950\end{array}$ & & $\begin{array}{l}1.0,=1 \\
7.5,=2 \\
5.6,=2\end{array}$ & $\begin{array}{r}.372 \\
.372 \\
.262\end{array}$ \\
\hline
\end{tabular}

\begin{tabular}{c|c|c|c|c|c}
\hline$r / a$ & $\frac{T t}{r^{28}}$ & $1-\frac{s}{s_{0}}$ & $r / a$ & $\frac{T t}{r^{2}}$ & $1-\frac{s}{s_{0}}$ \\
$\theta_{0}=120^{\circ} ; \frac{\theta}{\theta_{0}}=0.45-$ Continued
\end{tabular}

\begin{tabular}{l|l|l||l|l|r}
\hline 0.70 & $4.2,-2$ & 0.174 & 0.80 & $7.5,-2$ & 0.628 \\
& $3.2,-2$ & .110 & & $5,-2$ & .531 \\
& $2.4,-2$ & .061 & & $4.2,-2$ & .443 \\
.80 & $1.8,-2$ & .029 & & $3.2,-2$ & .364 \\
& $3.2,-1$ & .984 & & $.4,-2$ & .285 \\
& $2.4,-1$ & .955 & & $1.8,-2$ & .210 \\
& $1.8,-1$ & .902 & & $1.3,-2$ & .136 \\
& $1.3,-1$ & .814 & & $7.0,-2$ & .086 \\
& $1.0,-1$ & .727 & & & .046 \\
\hline
\end{tabular}

[Pl. 70]

\begin{tabular}{|c|c|c|c|c|c|}
\hline 0.05 & $\begin{array}{l}4.2,1 \\
3.2,1 \\
2.4,1 \\
1.8,1 \\
1.3,1 \\
1.0,1 \\
7.5,0 \\
5.6,0 \\
4.2,0\end{array}$ & $\begin{array}{r}0.990 \\
.983 \\
.975 \\
.966 \\
.955 \\
.945 \\
.942 \\
.916 \\
.996\end{array}$ & 0.40 & $\begin{array}{l}1.3, \mathrm{C} \\
1.0, \mathrm{C} \\
7.5,-1 \\
5.6,-1 \\
4.2,-1 \\
3.2,-1 \\
2.4,-1 \\
1.8,-1 \\
1.3,-1 \\
1.0,-1\end{array}$ & $\begin{array}{r}0.979 \\
. .944 \\
. .775 \\
.6640 \\
. .510 \\
.377 \\
.262 \\
.157 \\
.094\end{array}$ \\
\hline \multirow[t]{2}{*}{.10} & $\begin{array}{l}\text { 1. } 3,1 \\
\text { 1. } 0,1\end{array}$ & $\begin{array}{l}.982 \\
.968\end{array}$ & & $7.5,-2$ & .046 \\
\hline & $\begin{array}{l}7.5,0 \\
5.6,0 \\
4.2,0 \\
3.2,0 \\
2.4,0 \\
1.8,0 \\
1.3,0\end{array}$ & $\begin{array}{l}.947 \\
.923 \\
.899 \\
.874 \\
.844 \\
.808 \\
.759\end{array}$ & \multirow[t]{2}{*}{.45} & $\begin{array}{l}1.0,0 \\
7 ., 5,-1 \\
5.6,-1 \\
4.2,-1 \\
3.2,-1 \\
2.4,-1 \\
1.8,-1 \\
1.3,-1\end{array}$ & $\begin{array}{l}.974 \\
.928 \\
.845 \\
.726 \\
.591 \\
.442 \\
.304 \\
.177\end{array}$ \\
\hline \multirow[t]{2}{*}{.15} & $\begin{array}{l}7.5,0 \\
5.6,0\end{array}$ & $\begin{array}{l}.986 \\
.966 \\
.936\end{array}$ & & $\begin{array}{l}1.0,-1 \\
7.5,-2\end{array}$ & $\begin{array}{l}.102 \\
.049\end{array}$ \\
\hline & $\begin{array}{l}4.2,0 \\
3.2,0 \\
2.4,0 \\
1.8,0 \\
1.3,0 \\
1.0,0 \\
7.5,-1\end{array}$ & $\begin{array}{l}.856 \\
.899 \\
.857 \\
.860 \\
.7612 \\
.651\end{array}$ & \multirow[t]{2}{*}{.50} & $\begin{array}{l}1.0, C \\
7.5,-1 \\
5.6,-1 \\
4.2,-1 \\
3.2,-1 \\
2.4,-1 \\
1.8,-1\end{array}$ & $\begin{array}{l}.990 \\
.9964 \\
.905 \\
.807 \\
.682 \\
.530 \\
.375\end{array}$ \\
\hline \multirow[t]{2}{*}{.20} & $\begin{array}{l}4.2,0 \\
3.2,0 \\
2.4,0 \\
1.8,0\end{array}$ & $\begin{array}{l}.978 \\
.951 \\
.907 \\
.851\end{array}$ & & $\begin{array}{l}1.3,-1 \\
1.0,-1 \\
7.5,-2 \\
5.6,-2\end{array}$ & $\begin{array}{l}.223 \\
.229 \\
.060 \\
.022\end{array}$ \\
\hline & $\begin{array}{l}1.3,0 \\
1.0,0 \\
7.5,-1 \\
5.6,-1 \\
4.2,-1\end{array}$ & $\begin{array}{l}.779 \\
.7203 \\
.580 \\
.501\end{array}$ & \multirow[t]{2}{*}{.60} & $\begin{array}{l}\text { 5. } 6,-1 \\
\text { 4. } 2,-1 \\
3.2,-1 \\
2.4,-1 \\
1.8,-1\end{array}$ & $\begin{array}{r}.973 \\
.924 \\
.844 \\
.721 \\
573\end{array}$ \\
\hline \multirow[t]{2}{*}{.25} & $\begin{array}{l}3.2,0 \\
2.4,0 \\
1.8,0 \\
1.3,0 \\
1.0,0\end{array}$ & $\begin{array}{l}.985 \\
.958 \\
.911 \\
.834 \\
.760 \\
.767\end{array}$ & & $\begin{array}{l}\text { 1. } 0,-1 \\
1.3,-1 \\
\text { 7. } 0,-1 \\
5.6,-2 \\
4.2,-2\end{array}$ & $\begin{array}{l}.070 \\
.397 \\
.269 \\
.157 \\
.078 \\
.033\end{array}$ \\
\hline & $\begin{array}{l}\text { 7. } 5,-1 \\
\text { 5. } 6,-1 \\
\text { 4. } 2,-1 \\
\text { 3. } 2,-1 \\
2.4,-1\end{array}$ & $\begin{array}{r}.675 \\
.588 \\
.503 \\
.420 \\
.330\end{array}$ & \multirow[t]{2}{*}{.70} & $\begin{array}{l}\text { 4. } 2,-1 \\
\text { 3. } 2,-1 \\
\text { 1. } 4,-1 \\
1.8,-1 \\
1.3-1\end{array}$ & $\begin{array}{l}.978 \\
.941 \\
.870 \\
.766\end{array}$ \\
\hline \multirow[t]{2}{*}{.30} & $\begin{array}{l}2.4,0 \\
1.8,0 \\
1.3,0 \\
1.0,0 \\
7.5,-1 \\
\text { 5. },-1 \\
\text { 4. },-1\end{array}$ & $\begin{array}{l}.986 \\
.959 \\
.899 \\
.827 \\
.730 \\
.623 \\
.520 \\
.96\end{array}$ & & $\begin{array}{l}\text { 1. } 0,-1 \\
\text { 7. } 0,-2 \\
\text { 5. } 6,-2 \\
\text { 4. } 2,-2 \\
\text { 3. } 2,-2 \\
\text { 2. } 4,-2 \\
\text { 1. } 8,-2\end{array}$ & $\begin{array}{l}.020 \\
.496 \\
.369 \\
.259 \\
.173 \\
.110 \\
.061 \\
.029\end{array}$ \\
\hline & $\begin{array}{l}3.2,-1 \\
\text { 1. } 8,-1 \\
1.3,-1\end{array}$ & $\begin{array}{l}.426 \\
.332 \\
.242 \\
.152\end{array}$ & \multirow[t]{2}{*}{.80} & $\begin{array}{l}\text { 3. } 2,-1 \\
2.4,-1 \\
\text { 1. } 8,-1\end{array}$ & $\begin{array}{r}.984 \\
.954 \\
.9901\end{array}$ \\
\hline .35 & $\begin{array}{l}1.8,0 \\
1.3,0 \\
\text { 1. } 0,0 \\
7.5,-1 \\
\text { 5. } 6,-1 \\
4.2,-1 \\
\text { 3. } 2,-1 \\
2.4,-1 \\
1.8,-1 \\
1.3,-1 \\
1.0,-1\end{array}$ & $\begin{array}{l}.985 \\
.949 \\
.894 \\
.804 \\
.689 \\
.566 \\
.453 \\
.343 \\
.245 \\
.152 \\
.093\end{array}$ & & $\begin{array}{l}\text { 1. } 0,-1 \\
7.5,-2 \\
5.6,-2 \\
4.2,-2 \\
3.2,-2 \\
2.4,-2 \\
1.8,-2 \\
1.3,-2 \\
1.0,-2 \\
7.5,-3\end{array}$ & $\begin{array}{l}.725 \\
.625 \\
.529 \\
.442 \\
.364 \\
.285 \\
.210 \\
.136 \\
.086 \\
.046\end{array}$ \\
\hline
\end{tabular}




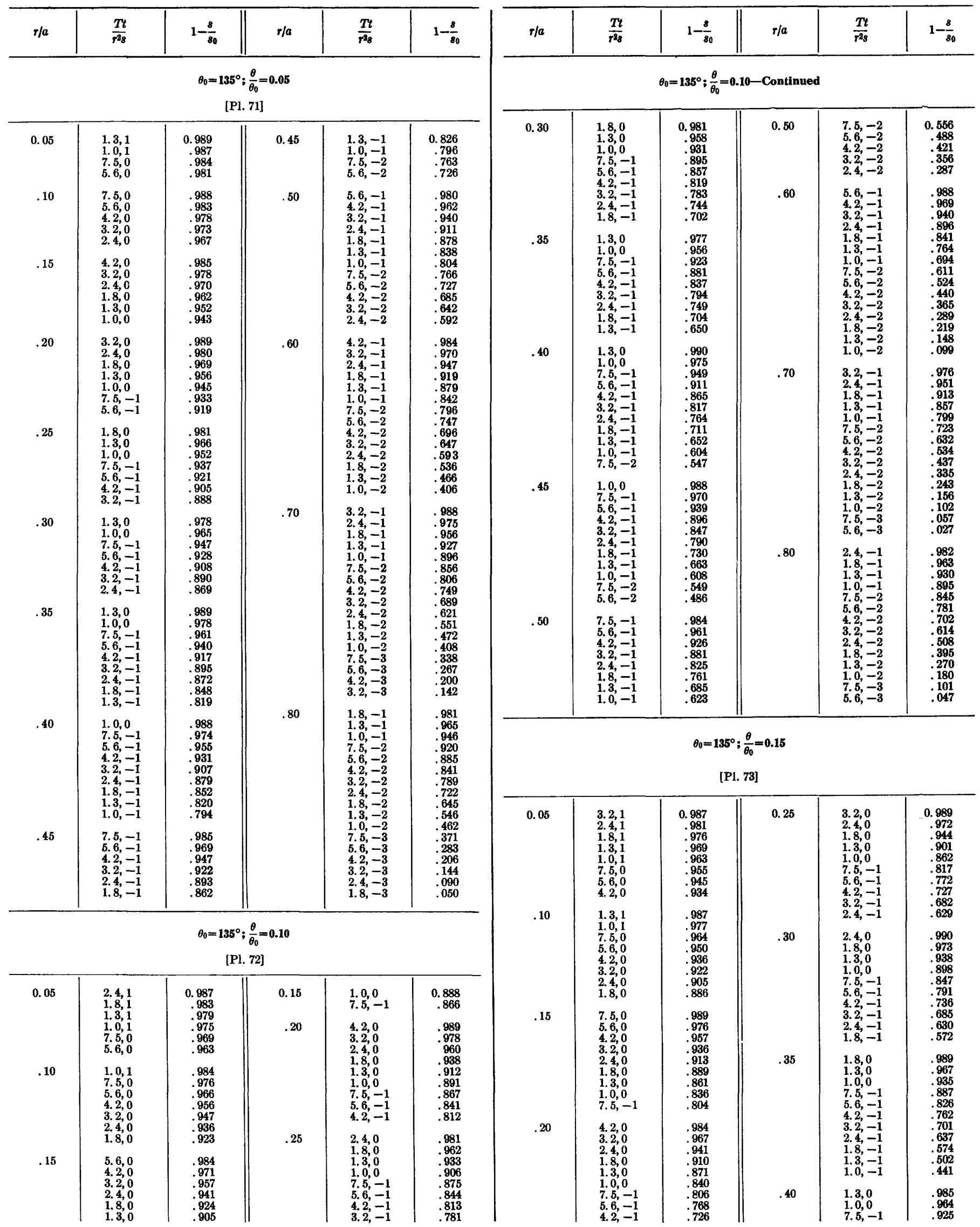




\begin{tabular}{|c|c|c|c|c|c|}
\hline$\tau / a$ & $\frac{T t}{r^{28}}$ & $1-\frac{8}{80}$ & $r / a$ & $\frac{T t}{r^{28}}$ & $1-\frac{8}{80}$ \\
\hline \multicolumn{6}{|c|}{$\theta_{0}=135^{\circ} ; \frac{\theta}{\theta_{0}}=0.15-$ Continued } \\
\hline 0.40 & $\begin{array}{l}5.6,-1 \\
4.2,-1 \\
3.2,-1 \\
2.4,-1 \\
1.8,-1 \\
1.3,-1 \\
1.0,-1 \\
7.5,-2\end{array}$ & $\begin{array}{r}0.869 \\
.802 \\
.733 \\
.658 \\
.585 \\
.505 \\
.442 \\
.372\end{array}$ & \multirow[t]{2}{*}{0.60} & \multirow[t]{2}{*}{$\begin{array}{l}1.8,-1 \\
1.3,-1 \\
1.0,-1 \\
7.5,-2 \\
5.6,-2 \\
4.2,-2 \\
3.2,-2 \\
2.4,-2 \\
1.8,-2 \\
1.3,-2\end{array}$} & \multirow[t]{2}{*}{$\begin{array}{r}0.768 \\
.661 \\
.566 \\
.458 \\
.351 \\
.257 \\
.181 \\
.117 \\
.069 \\
.032\end{array}$} \\
\hline .45 & $1.0,0$ & .982 & & & \\
\hline & $\begin{array}{l}\text { 5. } 6,-1 \\
4.2,-1 \\
3.2,-1 \\
2.4,-1 \\
1.8,-1 \\
1.3,-1 \\
1.0,-1 \\
7.5,-2 \\
5.6-2 \\
4.2,-2\end{array}$ & $\begin{array}{l}.900 \\
.910 \\
.777 \\
.695 \\
.611 \\
.519 \\
.448 \\
.374 \\
.302 \\
.232\end{array}$ & \multirow[t]{2}{*}{.70} & \multirow{2}{*}{$\begin{array}{l}\text { 4. } 2,-1 \\
3.2,-1 \\
2.4,-1 \\
1.8,-1 \\
1.3,-1 \\
1.0,-1 \\
7.5,-2 \\
5.6,-2 \\
4.2,-2 \\
3.2,-2 \\
2.4,-2 \\
1.8,-2 \\
1.3,-2\end{array}$} & $\begin{array}{l}.986 \\
.965 \\
.928 \\
.873 \\
.792 \\
.712 \\
.609 \\
.494 \\
.376 \\
.270\end{array}$ \\
\hline \multirow[t]{2}{*}{.50} & $\begin{array}{l}7.5,-1 \\
5.6,-1 \\
4.2,-1\end{array}$ & $\begin{array}{l}.976 \\
.943 \\
.891\end{array}$ & & & $\begin{array}{l}.096 \\
.041\end{array}$ \\
\hline & $\begin{array}{l}3.2,-1 \\
2.4,-1 \\
1.8,-1 \\
1.3,-1 \\
1.0,-1 \\
7.5,-2 \\
5.6,-2 \\
4.2,-2 \\
3.2,-2 \\
2.4,-2\end{array}$ & $\begin{array}{l}.826 \\
.745 \\
.655 \\
.550 \\
.468 \\
.384 \\
.305 \\
.233 \\
.171 \\
.114\end{array}$ & \multirow[t]{2}{*}{.80} & \multirow{2}{*}{$\begin{array}{l}3.2,-1 \\
2.4,-1 \\
1.8,-1 \\
1.3,-1 \\
1.0,-1 \\
7.5,-2 \\
5.6,-2 \\
4.2,-2 \\
3.2,-2 \\
2.4,-2 \\
1.8,-2 \\
1.3,-2 \\
1.0,-2 \\
7.5,-3 \\
5.6,-3\end{array}$} & $\begin{array}{l}.990 \\
.973 \\
.945 \\
.899 \\
.849 \\
.780 \\
. .694 \\
.596 \\
.493 \\
.379\end{array}$ \\
\hline .60 & $\begin{array}{l}\text { 5. } 6,-1 \\
4.2,-1 \\
3.2,-1 \\
2.4,-1\end{array}$ & $\begin{array}{r}.982 \\
.955 \\
.912 \\
.848\end{array}$ & & & $\begin{array}{l}.271 \\
.166 \\
.000 \\
.021\end{array}$ \\
\hline
\end{tabular}

$\theta_{0}=135^{\circ} ; \frac{\theta}{\theta_{0}}=0.20$

[P1. 74]

\begin{tabular}{|c|c|c|c|c|c|}
\hline \multirow[t]{2}{*}{0.05} & $\begin{array}{l}\text { 4. 2,1 } \\
\text { 3.2,1 }\end{array}$ & $\begin{array}{r}0.989 \\
.983\end{array}$ & 0.25 & $\begin{array}{l}\text { 3. } 2,-1 \\
2.4,-1\end{array}$ & $\begin{array}{r}0.593 \\
.529\end{array}$ \\
\hline & $\begin{array}{l}2.4,1 \\
1.8,1 \\
1.3,1 \\
1.0,1 \\
7.5,0 \\
5.6,0 \\
\mathbf{4 . 2 , 0}\end{array}$ & $\begin{array}{l}.976 \\
.968 \\
.960 \\
.952 \\
.942 \\
.929 \\
.915\end{array}$ & .30 & \multirow{2}{*}{$\begin{array}{l}2.4,0 \\
1.8,0 \\
1.3,0 \\
1.0,0 \\
7.5,-1 \\
5.6,-1 \\
4.2,-1 \\
3.2,-1 \\
2.4,-1 \\
1.8,-1\end{array}$} & $\begin{array}{l}.987 \\
.964 \\
.919 \\
.868 \\
.802 \\
.731 \\
.662\end{array}$ \\
\hline \multirow[t]{2}{*}{.10} & $\begin{array}{l}1.3,1 \\
1.0,1 \\
7,5\end{array}$ & $\begin{array}{r}.983 \\
.970 \\
.953\end{array}$ & & & $\begin{array}{l}.598 \\
.530 \\
.460\end{array}$ \\
\hline & $\begin{array}{l}\mathbf{5 . 6 , 0}, 0 \\
4.2,0 \\
3.2,0 \\
2.4,0 \\
1.8,0 \\
1.3,0\end{array}$ & $\begin{array}{l}.905 \\
.935 \\
.917 \\
.899 \\
.878 \\
.853 \\
.820\end{array}$ & \multirow[t]{2}{*}{.35} & \multirow{2}{*}{$\begin{array}{l}1.8,0 \\
1.3,0 \\
1.0,0 \\
7.5,-1 \\
5.6,-1 \\
4.2,-1 \\
3.2,-1 \\
2.4,-1 \\
1.8,-1 \\
1.3,-1 \\
1.0,-1\end{array}$} & $\begin{array}{l}.986 \\
.957 \\
.916 \\
.853 \\
.776 \\
.694\end{array}$ \\
\hline \multirow[t]{2}{*}{.15} & $\begin{array}{l}7.5,0 \\
5.6,0 \\
4.2,0 \\
3.2,0\end{array}$ & $\begin{array}{l}.986 \\
.969 \\
.945 \\
.918\end{array}$ & & & $\begin{array}{l}.038 \\
. .4363 \\
.380 \\
.313\end{array}$ \\
\hline & $\begin{array}{l}2.4,0 \\
1.8,0 \\
1.3,0 \\
1.0,0 \\
7.5,-1\end{array}$ & $\begin{array}{l}.887 \\
.856 \\
.820 \\
.788 \\
.748\end{array}$ & \multirow[t]{2}{*}{.40} & $\begin{array}{l}1.3,0 \\
1.0,0 \\
7.5,-1 \\
5.6,-1 \\
4,2,-1\end{array}$ & $\begin{array}{l}.981 \\
.953 \\
.903 \\
.831 \\
.745\end{array}$ \\
\hline \multirow[t]{2}{*}{.20} & $\begin{array}{l}4.2,0 \\
3.2,0 \\
2.4,0 \\
1.8,0 \\
1.3,0\end{array}$ & $\begin{array}{l}.979 \\
.957 \\
.924 \\
.883 \\
.834 \\
.704\end{array}$ & & $\begin{array}{l}\text { 3. } 2,-1 \\
2.4,-1 \\
1.8,-1 \\
1.3,-1 \\
\text { 1. } 0,-1 \\
7.5,-2\end{array}$ & $\begin{array}{l}.657 \\
.564 \\
.476 \\
.384 \\
.314 \\
.242\end{array}$ \\
\hline & $\begin{array}{l}7.5,-1 \\
5.6,-1 \\
4.2,-1\end{array}$ & $\begin{array}{r}.749 \\
.701 \\
.648\end{array}$ & \multirow[t]{2}{*}{.45} & \multirow{2}{*}{$\begin{array}{l}1.0,0 \\
7.5,-1 \\
5.6,-1 \\
4.2,-1 \\
3.2,-1 \\
2.4,-1 \\
1.8,-1 \\
1.3,-1 \\
1.0,-1 \\
7.5,-2 \\
5.6,-2 \\
4.2,-2\end{array}$} & $\begin{array}{l}.977 \\
.942 \\
.883\end{array}$ \\
\hline .25 & $\begin{array}{l}\text { 3. } 2,0 \\
2.4,0 \\
1.8,0 \\
1.3,0 \\
1.0,0 \\
7.5,-1 \\
5.6,-1 \\
4.2,-1\end{array}$ & $\begin{array}{l}.985 \\
.963 \\
.927 \\
.872 \\
.821 \\
.764 \\
.707 \\
.650\end{array}$ & & & $\begin{array}{l}.803 \\
.714 \\
.611 \\
.508 \\
.400 \\
.321 \\
.245 \\
.176 \\
.117\end{array}$ \\
\hline
\end{tabular}

\begin{tabular}{c|c|c||c|c|c}
\hline$\tau / a$ & $\frac{T t}{r^{2}}$ & $1-\frac{8}{80}$ & $r / a$ & $\frac{T t}{r^{2}}$ & $1-\frac{8}{8_{0}}$ \\
\hline$\theta_{0}=135^{\circ} ; \frac{\theta}{\theta_{0}}=0.20-$ Continued
\end{tabular}

\begin{tabular}{|c|c|c|c|c|c|}
\hline 0.50 & $\begin{array}{l}7.5,-1 \\
5.6,-1 \\
4.2,-1 \\
3.2,-1 \\
2.4,-1 \\
1.8,-1 \\
1.3,-1 \\
1.0,-1 \\
7.5,-2 \\
5.6,-2 \\
4.2,-2 \\
3.2,-2 \\
2.4,-2\end{array}$ & $\begin{array}{r}0.969 \\
.026 \\
.859 \\
.776 \\
.673 \\
.562 \\
.437 \\
.344 \\
.255 \\
.179 \\
.118 \\
.073 \\
.038\end{array}$ & 0.70 & $\begin{array}{l}\text { 4. } 2,-1 \\
3.2,-1 \\
2.4,-1 \\
1.8,-1 \\
1.3,-1 \\
1.0,-1 \\
7.5,-2 \\
5.6,-2 \\
4.2,-2 \\
3.2,-2 \\
2.4,-2 \\
1.8,-2\end{array}$ & $\begin{array}{r}0.981 \\
.955 \\
.906 \\
.837 \\
.736 \\
.639 \\
.520 \\
.395 \\
.276 \\
.178 \\
.098 \\
.045\end{array}$ \\
\hline .60 & $\begin{array}{l}5.6,-1 \\
4.2,-1 \\
3.2,-1 \\
\text {.24, -1 } \\
1.8,-1 \\
1.3,-1 \\
1.0,-1 \\
7.5,-2 \\
5.6,-2 \\
4.2,-2 \\
3.2,-2 \\
2.4,-2\end{array}$ & $\begin{array}{l}.977 \\
.941 \\
.886 \\
.805 \\
.704 \\
.573 \\
.461 \\
.341 \\
.232 \\
.144 \\
.083 \\
.041\end{array}$ & .00 & $\begin{array}{l}2.4,-1 \\
1.8,-1 \\
1.3,-1 \\
1.0,-1 \\
7.5,-2 \\
5.6,-2 \\
4.2,-2 \\
3.2,-2 \\
2.4,-2 \\
1.8,-2 \\
1.3,-2 \\
1.0,-2 \\
7.5,-3\end{array}$ & $\begin{array}{l}.965 \\
.929 \\
. .870 \\
.808 \\
.726 \\
.629 \\
.224 \\
.422 \\
.319 \\
.226 \\
.140 \\
.088 \\
.046\end{array}$ \\
\hline
\end{tabular}

$\theta_{0}=135^{\circ} ; \frac{\theta}{\theta_{0}}=0.25$

[Pl. 75]

\begin{tabular}{|c|c|c|c|c|c|}
\hline 0.05 & $\begin{array}{l}4.2,1 \\
3.2,1 \\
2.4,1 \\
1.8,1 \\
1.3,1 \\
1.0,1 \\
7.5,0 \\
5.6,0 \\
4.2,0\end{array}$ & $\begin{array}{r}0.987 \\
.979 \\
.971 \\
.962 \\
.951 \\
.942 \\
.930 \\
.915 \\
.898\end{array}$ & 0.35 & $\begin{array}{l}1.8,0 \\
1.3,0 \\
1.0,0 \\
7.5,-1 \\
5.6,-1 \\
4.2,-1 \\
3.2,-1 \\
2.4,-1 \\
1.8,-1\end{array}$ & $\begin{array}{r}0.983 \\
.948 \\
.889 \\
.824 \\
.772 \\
.635 \\
.545 \\
.456 \\
.373\end{array}$ \\
\hline .10 & $\begin{array}{l}1.3,1 \\
1.0,1 \\
7.5,0 \\
\mathbf{5 . 6 , 0} \\
\mathbf{4 . 2 , 0} \\
\mathbf{3 . 2 , 0} \\
2.4,0 \\
\mathbf{1 . 8 , 0} \\
1.3,0\end{array}$ & $\begin{array}{l}.979 \\
.964 \\
.944 \\
.922 \\
.900 \\
.878 \\
.853 \\
.823 \\
.784\end{array}$ & \multirow[t]{2}{*}{.40} & $\begin{array}{l}\text { 1. } 0,-1 \\
1.3,0 \\
1.0,0 \\
7.5,-1 \\
5.6,-1 \\
4.2,-1 \\
3.2,-1 \\
2.4,-1 \\
1.8,-1\end{array}$ & $\begin{array}{l}.218 \\
.977 \\
.944 \\
.884 \\
.797 \\
.695 \\
.592 \\
. .485 \\
.387\end{array}$ \\
\hline .15 & $\begin{array}{l}7.5,0 \\
5.6,0 \\
4,2,0\end{array}$ & $\begin{array}{l}.983 \\
.963 \\
.934\end{array}$ & & $\begin{array}{l}1.3,-1 \\
\text { 1. } 0,-1 \\
7.5,-2\end{array}$ & $\begin{array}{r}.289 \\
.220 \\
.153\end{array}$ \\
\hline & $\begin{array}{l}4.2,0 \\
3.2,0 \\
2.4,0 \\
1.8,0 \\
1.3,0 \\
1.0,0 \\
7.5,-1\end{array}$ & $\begin{array}{l}.934 \\
.901 \\
.864 \\
.7827 \\
.746 \\
.698\end{array}$ & \multirow[t]{2}{*}{.45} & $\begin{array}{l}1.0,0 \\
7.5,-1 \\
5.6,-1 \\
4.2,-1 \\
3.2,-1 \\
2.4,-1 \\
1.8,-1\end{array}$ & $\begin{array}{l}.973 \\
.931 \\
.860 \\
.764 \\
.658 \\
. .539 \\
.424\end{array}$ \\
\hline .20 & $\begin{array}{l}4.2,0 \\
3.2,0 \\
2.4,0 \\
1.8,0 \\
1.3,0\end{array}$ & $\begin{array}{l}.975 \\
.949 \\
.909 \\
.859 \\
.800\end{array}$ & & $\begin{array}{l}\text { 1. } 3,-1 \\
1.0,-1 \\
7.5,-2 \\
5.6,-2 \\
4.2,-2\end{array}$ & $\begin{array}{l}.3207 \\
.227 \\
.155 \\
.098 \\
.055\end{array}$ \\
\hline & $\begin{array}{l}1.0,0 \\
7.5,-1 \\
5.6,-1 \\
4.2,-1\end{array}$ & $\begin{array}{l}.753 \\
.700 \\
.643 \\
.581\end{array}$ & \multirow[t]{2}{*}{.50} & \multirow{2}{*}{$\begin{array}{l}1.0,0 \\
7.5,-1 \\
5.6,-1 \\
4.2,-1 \\
3.2,-1 \\
2.4-1 \\
1.8,-1 \\
1.3,-1 \\
1.0,-1 \\
7.5,-2 \\
5.6-2 \\
4.2,-2 \\
3.2,-2\end{array}$} & $\begin{array}{l}.988 \\
.963 \\
.911\end{array}$ \\
\hline .25 & $\begin{array}{l}3.2,0 \\
2.4,0 \\
1.8,0 \\
1.3,0 \\
1.0,0 \\
7.5,-1 \\
5.6,-1 \\
4.2,-1 \\
3.2,-1 \\
2.4,-1\end{array}$ & $\begin{array}{l}.982 \\
.956 \\
.913 \\
.846 \\
.785 \\
.717 \\
.650 \\
.583 \\
.518 \\
.445\end{array}$ & & & $\begin{array}{r}.732 \\
.611 \\
.485 \\
.348 \\
.252 \\
.167 \\
.101 \\
.056 \\
.028 \\
070\end{array}$ \\
\hline .30 & $\begin{array}{l}2.4,0 \\
1.8,0 \\
1.3,0 \\
1.0,0 \\
7.5,-1 \\
5.6,-1 \\
4.2,-1 \\
3.2,-1 \\
2.4,-1 \\
1.8,-1\end{array}$ & $\begin{array}{l}.984 \\
.957 \\
.903 \\
.841 \\
.762 \\
.678 \\
.597 \\
.523 \\
.446 \\
.370\end{array}$ & .60 & 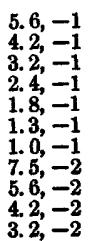 & $\begin{array}{l}.929 \\
.863 \\
.767 \\
.649 \\
.501 \\
.381 \\
.259 \\
.156 \\
.083 \\
.039\end{array}$ \\
\hline
\end{tabular}




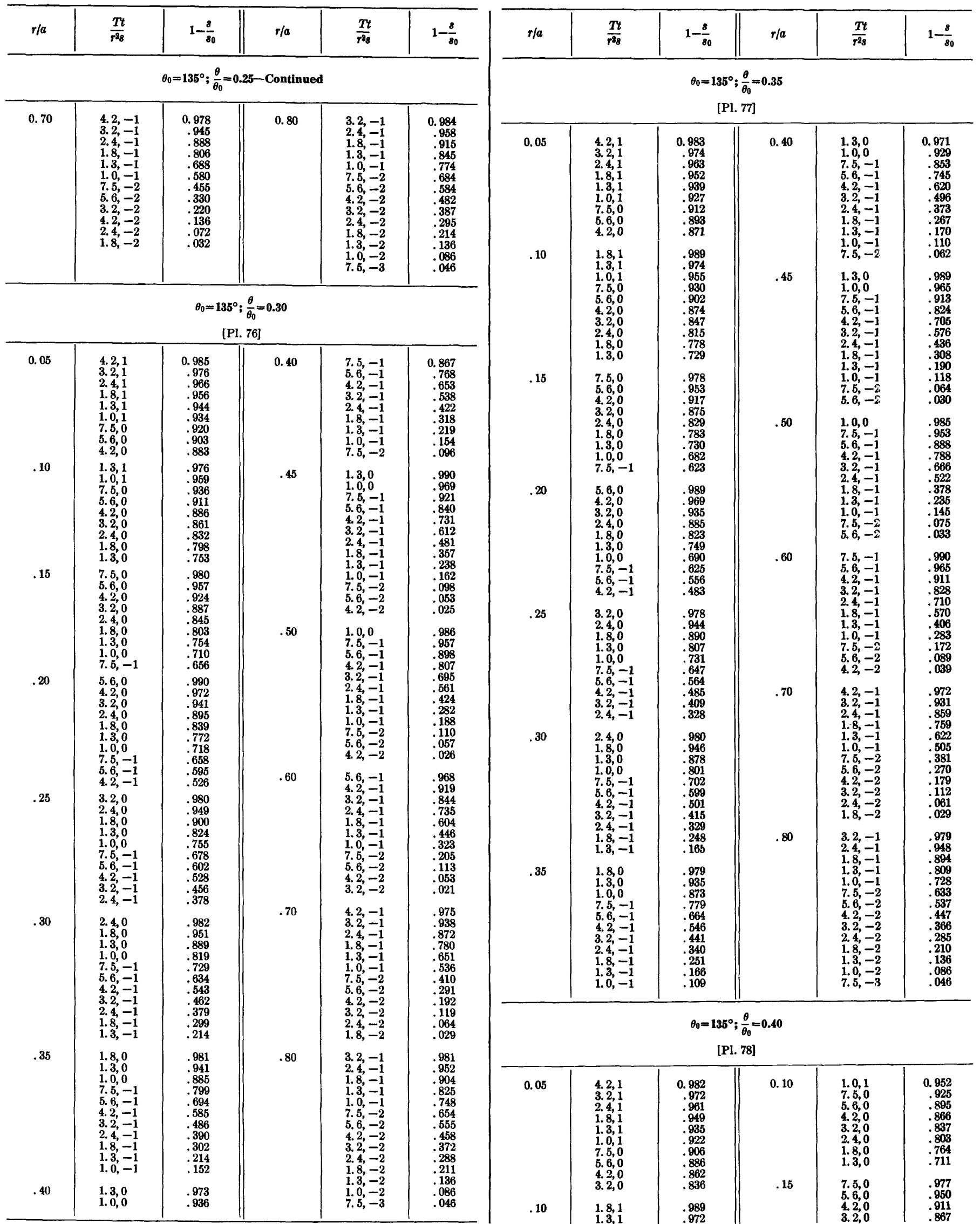


TABLES

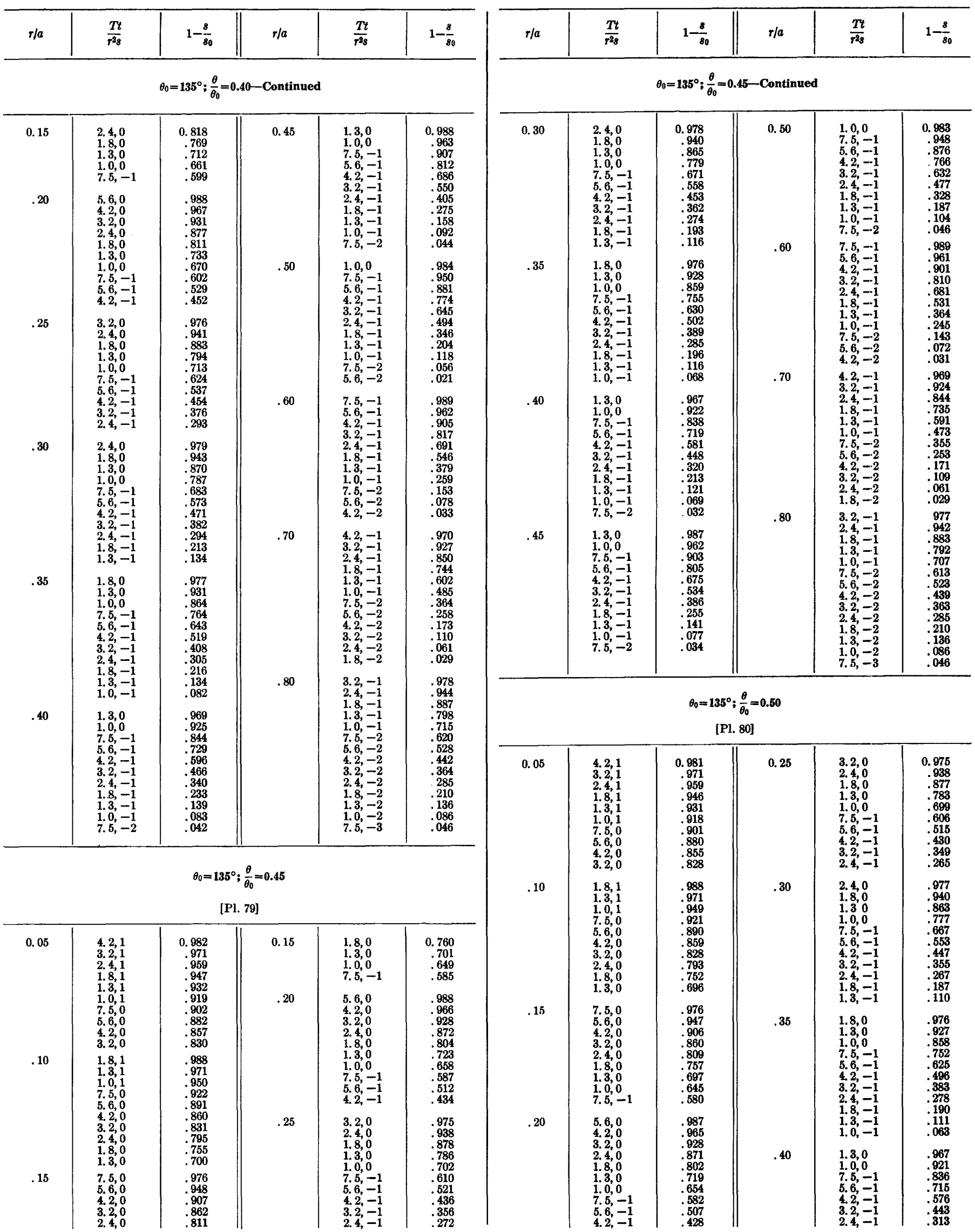




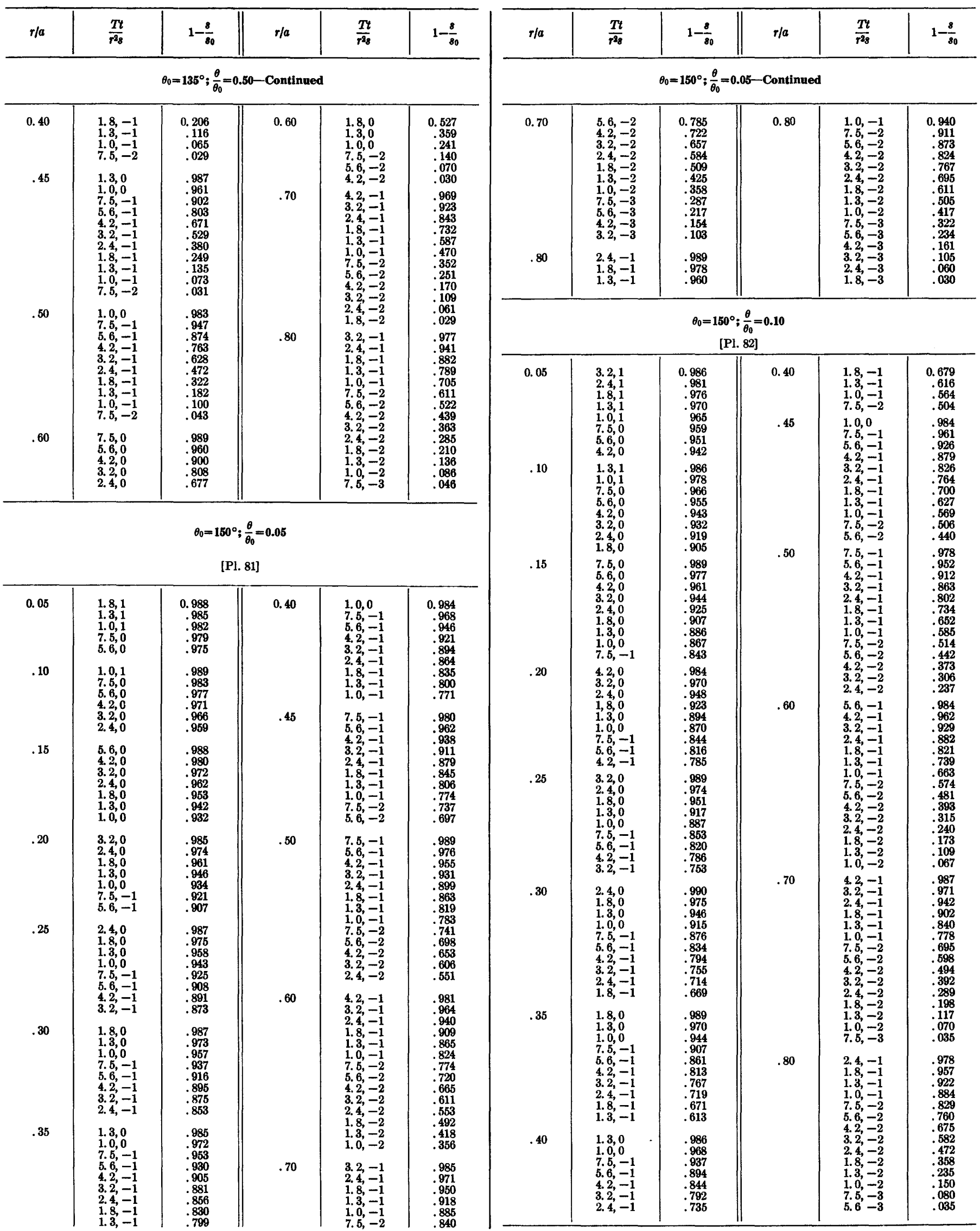




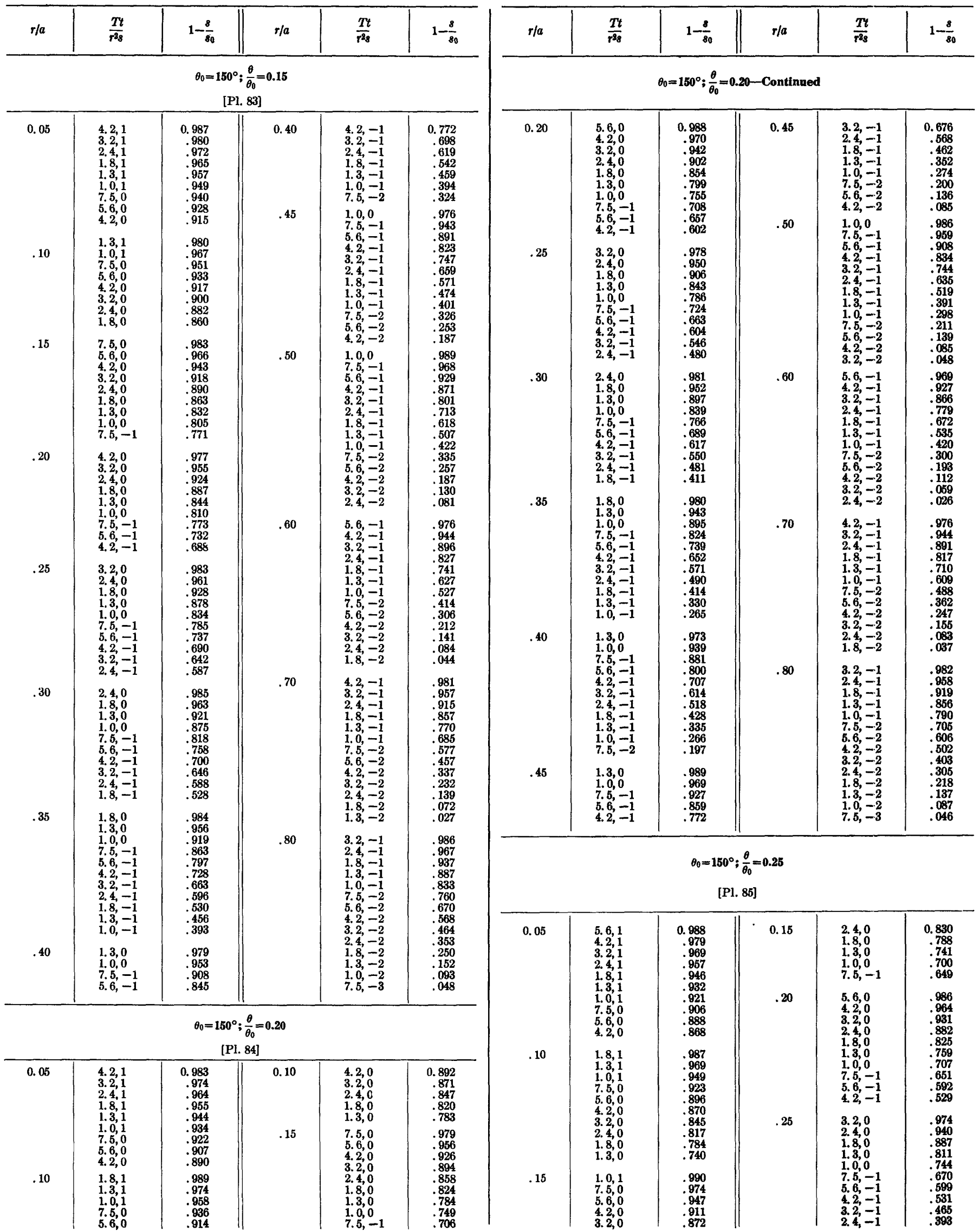


HYDRAULIC DIFFUSIVITY, WEDGE-SHAPED AQUIFERS DRAINED BY STREAMS

\begin{tabular}{|c|c|c|c|c|c|c|c|c|c|c|c|}
\hline$r / a$ & $\frac{T t}{r^{2} s}$ & $1-\frac{8}{80}$ & $r / a$ & $\frac{T t}{T^{2}}$ & $1-\frac{8}{80}$ & $r / a$ & $\frac{T t}{r^{28}}$ & $1-\frac{8}{80}$ & $r / a$ & $\frac{T t}{r^{2 s}}$ & $1-\frac{8}{80}$ \\
\hline \multicolumn{6}{|c|}{$\theta_{0}=150^{\circ} ; \frac{\theta}{\theta_{0}}=0.25-$ Continued } & \multicolumn{6}{|c|}{$\theta_{0}=150^{\circ} ; \frac{\theta}{\theta_{0}}=0.30-$ Continued } \\
\hline 0.30 & $\begin{array}{l}2.4,0 \\
1.8,0 \\
1.3,0 \\
1.0,0 \\
7.5,-1 \\
5.6,-1 \\
4.2,-1 \\
3.2,-1 \\
2.4,-1 \\
1.8,-1\end{array}$ & $\begin{array}{r}0.977 \\
.942 \\
.877 \\
.807 \\
.720 \\
.630 \\
.545 \\
.470 \\
.394 \\
.319\end{array}$ & 0.50 & $\begin{array}{l}1.0,0 \\
7.5,-1 \\
5.6,-1 \\
4.2,-1 \\
3.2,-1 \\
2.4,-1 \\
1.8,-1 \\
1.3,-1 \\
1.0,-1 \\
7.5,-2 \\
5.6,-2\end{array}$ & $\begin{array}{r}0.983 \\
.951 \\
.890 \\
.801 \\
.695 \\
.569 \\
.440 \\
.304 \\
.211 \\
.131 \\
.073\end{array}$ & 0.40 & $\begin{array}{l}1.3,0 \\
1.0,0 \\
7.5,-1 \\
5.6,-1 \\
4.2,-1 \\
3.2,-1 \\
2.4,-1 \\
1.8,-1 \\
1.3,-1 \\
1.0,-1 \\
7.5,-2\end{array}$ & $\begin{array}{r}0.963 \\
.916 \\
.836 \\
.727 \\
.605 \\
.487 \\
.370 \\
.269 \\
.177 \\
.118 \\
.069\end{array}$ & 0.60 & $\begin{array}{l}4.2,-1 \\
3.2,-1 \\
2.4,-1 \\
1.8,-1 \\
1.3,-1 \\
1.0,-1 \\
7.5,-2 \\
5.6,-2 \\
4,2,-2\end{array}$ & $\begin{array}{r}0.900 \\
.817 \\
.702 \\
.568 \\
.410 \\
.290 \\
.179 \\
.095 \\
.043\end{array}$ \\
\hline .35 & $\begin{array}{l}1.8,0 \\
1.3,0 \\
1.0,0 \\
7.5,-1 \\
5.6,-1 \\
4.2,-1 \\
3.2,-1 \\
.4,-1 \\
1.8,-1 \\
1.3,-1 \\
1.0,-1 \\
7.5,-2 \\
5,6,-2 \\
4,\end{array}$ & $\begin{array}{l}.976 \\
.932 \\
.873 \\
.788 \\
.688 \\
.586 \\
.494 \\
.404 \\
.322 \\
.238 \\
.176 \\
.117 \\
.069\end{array}$ & .60 & $\begin{array}{l}\text { 4. } 2,-2 \\
7.5,-1 \\
5.6,-1 \\
4.2,-1 \\
3.2,-1 \\
2.4,-1 \\
1.8,-1 \\
1.3,-1 \\
1.0,-1 \\
7.5,-2 \\
5.6,-2 \\
4.2,-2 \\
\text { 3. } 2,-2\end{array}$ & $\begin{array}{l}.037 \\
.988 \\
.963 \\
.913 \\
.839 \\
.737 \\
.614 \\
.463 \\
.343 \\
.225 \\
.129 \\
.063 \\
.027\end{array}$ & .45 & $\begin{array}{l}1.3,0 \\
1.0,0 \\
7.5,-1 \\
5.6,-1 \\
4.2,-1 \\
3.2,-1 \\
2.4,-1 \\
1.8,-1 \\
1.3,-1 \\
1.0,-1 \\
7.5,-2 \\
5.6,-2\end{array}$ & $\begin{array}{l}.984 \\
.957 \\
.899 \\
.807 \\
.690 \\
.565 \\
.432 \\
.310 \\
.196 \\
.126 \\
.072 \\
.035\end{array}$ & .70 & $\begin{array}{l}5.6,-1 \\
4.2,-1 \\
3.2,-1 \\
2.4,-1 \\
1.8,-1 \\
1.3,-1 \\
1.0,-1 \\
7.5,-2 \\
5.6,-2 \\
4.2,-2 \\
\text {.. } 2,-2 \\
2.4,-2 \\
1.8,-2\end{array}$ & $\begin{array}{l}.989 \\
.966 \\
.923 \\
.851 \\
.754 \\
.623 \\
.510 \\
.388 \\
.275 \\
.182 \\
.114 \\
.062 \\
.029\end{array}$ \\
\hline .40 & $\begin{array}{l}4,2,-2 \\
1.3,0 \\
1.0,0 \\
7.5,-1 \\
5.6,-1 \\
4,2,-1 \\
3.2,-1 \\
2.4,-1 \\
1.8,-1 \\
1.3,-1 \\
1.0,-1 \\
7.5,-2\end{array}$ & $\begin{array}{l}.036 \\
.967 \\
.927 \\
.857 \\
.761 \\
.651 \\
.544 \\
.435 \\
.337 \\
.242 \\
.177 \\
.117\end{array}$ & .70 & $\begin{array}{l}3.2,-2 \\
4.2,-1 \\
3.2,-1 \\
2.4,-1 \\
1.8,-1 \\
1.3,-1 \\
1.0,-1 \\
7.5,-2 \\
5.6,-2 \\
4.2,-2 \\
3.2,-2 \\
2.4,-2 \\
1.8,-2\end{array}$ & $\begin{array}{l}.027 \\
.971 \\
.933 \\
.869 \\
.783 \\
.661 \\
.551 \\
.427 \\
.306 \\
.202 \\
.124 \\
.066 \\
.030\end{array}$ & .50 & $\begin{array}{l}1.0,0 \\
7.5,-1 \\
5.6,-1 \\
4.2,-1 \\
3.2,-1 \\
2.4,-1 \\
1.8,-1 \\
1.3,-1 \\
1.0,-1 \\
7.5,-2 \\
5.6,-2\end{array}$ & $\begin{array}{l}.980 \\
.943 \\
.874 \\
.773 \\
.655 \\
.516 \\
.379 \\
.241 \\
.153 \\
.083 \\
.039\end{array}$ & \multirow[t]{2}{*}{.80} & \multirow[t]{2}{*}{$\begin{array}{l}3.2,-1 \\
2.4,-1 \\
1.8,-1 \\
1.3,-1 \\
1.0,-1 \\
7.5,-2 \\
5.6,-2 \\
4.2,-2 \\
3.2,-2 \\
2.4,-2 \\
1.8,-2 \\
1.3,-2 \\
1.0,-2 \\
7.5,-3\end{array}$} & \multirow[t]{2}{*}{$\begin{array}{l}.976 \\
.942 \\
.889 \\
.088 \\
.730 \\
.638 \\
.542 \\
.450 \\
.268 \\
.286 \\
.210 \\
.136 \\
.086 \\
.046\end{array}$} \\
\hline \multirow[t]{4}{*}{.45} & $\begin{array}{l}1.3,0 \\
1.0,0\end{array}$ & $\begin{array}{l}.986 \\
.962\end{array}$ & \multirow[t]{4}{*}{.80} & $\begin{array}{l}\text { 3. } 2,-1 \\
2.4,-1\end{array}$ & $\begin{array}{l}.979 \\
.949\end{array}$ & .60 & $\begin{array}{l}7.5,-1 \\
5.6,-1\end{array}$ & $\begin{array}{l}.986 \\
.957\end{array}$ & & & \\
\hline & $\begin{array}{l}\text { 5. } 6,-1 \\
4,2,=1 \\
3.2,=1 \\
\text { 2. } 4,-1\end{array}$ & $\begin{array}{l}.912 \\
.831 \\
.727 \\
.615 \\
.492\end{array}$ & & $\begin{array}{l}1.3,-1 \\
1.0,-1 \\
7.5,-2 \\
5.6,-2\end{array}$ & $\begin{array}{l}.829 \\
.756 \\
.665 \\
.566 \\
467\end{array}$ & \multicolumn{6}{|c|}{$\begin{array}{c}\theta_{0}=150^{\circ} ; \frac{\theta}{\theta_{0}}=0.35 \\
{[\text { PI. 87] }}\end{array}$} \\
\hline & $\begin{array}{l}1.0,-1 \\
1.0,=1 \\
\text { 7. } 0,=2 \\
5.6,=2 \\
4.2,-2\end{array}$ & $\begin{array}{l}.070 \\
.261 \\
.185 \\
.119 \\
.070 \\
.036\end{array}$ & & $\begin{array}{l}3.2,-2 \\
2,4,-2 \\
1.8,-2 \\
1.3,-2 \\
1.0,-2\end{array}$ & $\begin{array}{l}.377 \\
.290 \\
.212 \\
.136 \\
.086\end{array}$ & \multirow[t]{3}{*}{0.05} & \multirow{3}{*}{$\begin{array}{l}5.6,1 \\
4.2,1 \\
3.2,1 \\
2.4,1 \\
1.8,1 \\
1.3,1 \\
1.0,1 \\
7.5,0 \\
5.6,0 \\
4.2,0 \\
3.2,0\end{array}$} & \multirow{3}{*}{$\begin{array}{r}0.985 \\
.974 \\
.961 \\
.946 \\
.931 \\
.915 \\
.900 \\
.882 \\
.859 \\
.833 \\
.805\end{array}$} & 0.25 & $\begin{array}{l}5.6,-1 \\
4,2,-1 \\
3.2,-1 \\
2.4,-1\end{array}$ & $\begin{array}{l}0.505 \\
.426 \\
.352 \\
.275\end{array}$ \\
\hline & & & & $7.5,-3$ & .046 & & & & .30 & $\begin{array}{l}2.4,0 \\
1.8,0\end{array}$ & $\begin{array}{l}.971 \\
.927\end{array}$ \\
\hline & & $\theta_{0}=1$ & & & & & & & & $\begin{array}{l}1.3,0 \\
1.0,0 \\
7.5,-1 \\
5.6,-1\end{array}$ & $\begin{array}{l}.845 \\
.758 \\
.650 \\
.542\end{array}$ \\
\hline 0.05 & $\begin{array}{l}\text { 5. } 6,1 \\
4.2,1 \\
3.2,1 \\
2.4,1\end{array}$ & $\begin{array}{r}0.987 \\
.9976 \\
.964 \\
.951\end{array}$ & 0.20 & $\begin{array}{l}1.0,0 \\
7.5,-1 \\
5.6,-1 \\
4.2,-1\end{array}$ & $\begin{array}{r}0.667 \\
.604 \\
.539 \\
.470\end{array}$ & .10 & $\begin{array}{l}\text { 1. } 8,1 \\
\text { 1. } 3,1 \\
1.0,1 \\
7.5,0\end{array}$ & $\begin{array}{l}.983 \\
.961 \\
.936 \\
.903\end{array}$ & & $\begin{array}{l}3.2,-1 \\
2.4,-1 \\
1.8,-1 \\
1.3,-1\end{array}$ & $\begin{array}{l}.358 \\
.276 \\
.202 \\
.128\end{array}$ \\
\hline & 1. 8,1 & .938 & & $1,2,-1$ & .700 & & 5. 6,0 & .870 & .35 & $\begin{array}{l}1.8,0 \\
1,0\end{array}$ & $\begin{array}{r}.969 \\
914\end{array}$ \\
\hline & $\begin{array}{l}1.3,1 \\
1.0,1 \\
7.5,0 \\
5.6,0 \\
4.2,0 \\
3.2,0\end{array}$ & $\begin{array}{l}.923 \\
.909 \\
.892 \\
.872 \\
.849 \\
.823\end{array}$ & .25 & $\begin{array}{l}4.2,0 \\
3.2,0 \\
2.4,0 \\
1.8,0 \\
1.3,0 \\
1.0,0\end{array}$ & $\begin{array}{l}.990 \\
.970 \\
.931 \\
.871 \\
.784 \\
.708\end{array}$ & & $\begin{array}{l}4.2,0 \\
3.2,0 \\
2.4,0 \\
1.8,0 \\
1.3,0\end{array}$ & $\begin{array}{r}.837 \\
.806 \\
.770 \\
.729 \\
.675\end{array}$ & & $\begin{array}{l}1.3,0 \\
1.0,0 \\
7.5,-1 \\
5.6,-1 \\
4.2,-1 \\
3.2,-1\end{array}$ & $\begin{array}{l}.914 \\
.841 \\
.735 \\
.612 \\
.491 \\
.385 \\
.897\end{array}$ \\
\hline .10 & $\begin{array}{l}1.8,1 \\
1.3,1 \\
1.0,1 \\
7.5,0\end{array}$ & $\begin{array}{l}.985 \\
.965 \\
.942 \\
.912\end{array}$ & & $\begin{array}{l}\text { 7.5, -1 } \\
5.6,-1 \\
4.2,-1 \\
3.2,-1 \\
2.4,-1\end{array}$ & $\begin{array}{l}.625 \\
.546 \\
.471 \\
.401 \\
.325\end{array}$ & .15 & $\begin{array}{l}1.0,1 \\
7.5,0 \\
5.6,0 \\
4.2,0 \\
3.2,0\end{array}$ & $\begin{array}{l}.987 \\
.968 \\
.934 \\
.888 \\
.839\end{array}$ & & $\begin{array}{l}2.4,-1 \\
1.8,-1 \\
1.3,-1 \\
1.0,-1\end{array}$ & $\begin{array}{l}.287 \\
.205 \\
.129 \\
.081\end{array}$ \\
\hline & $\begin{array}{l}5.6,0 \\
4.2,0\end{array}$ & .881 & 30 & 40 & .020 & & $2.4,0$ & .786 & .40 & $\begin{array}{l}1.8,0 \\
1.3,0\end{array}$ & $\begin{array}{r}.989 \\
.959\end{array}$ \\
\hline & $\begin{array}{l}\text { 3. } 2,0 \\
2.4,0 \\
1.8,0 \\
1.3,0\end{array}$ & $\begin{array}{l}.802 \\
.823 \\
.791 \\
.753 \\
.704\end{array}$ & .30 & $\begin{array}{l}1.4,0 \\
1.3,0 \\
1.0,0 \\
7.5,-1\end{array}$ & $\begin{array}{l}.973 \\
.934 \\
.859 \\
.779 \\
.681\end{array}$ & & $\begin{array}{l}\text { l. } \\
\text { 1. } \\
\text { 1. } 0,0 \\
\text { 7. } 5,-1\end{array}$ & $\begin{array}{l}.676 \\
.626 \\
.565\end{array}$ & & $\begin{array}{l}1.0,0 \\
7.5,-1 \\
5.6=1 \\
4.2,-1\end{array}$ & $\begin{array}{l}.908 \\
.820 \\
.701 \\
.568\end{array}$ \\
\hline .15 & $\begin{array}{l}1.3,0 \\
1.0,1 \\
7.5,0 \\
5.6,0 \\
4.2,0 \\
3.2,0\end{array}$ & $\begin{array}{l}.704 \\
.989 \\
.971 \\
.940 \\
.899 \\
.854\end{array}$ & & $\begin{array}{l}\text { 5. } 6,-1 \\
\text { 4. } 2,-1 \\
\text { 3. } 2,-1 \\
\text { 2. } 4,-1 \\
1.8,-1 \\
1.3,-1\end{array}$ & $\begin{array}{l}.081 \\
.580 \\
.487 \\
.406 \\
.326 \\
.250 \\
.172\end{array}$ & .20 & $\begin{array}{l}5.6,0 \\
4.2,0 \\
3.2,0 \\
2.4,0 \\
1.8,0 \\
1.3,0\end{array}$ & $\begin{array}{l}.983 \\
.955 \\
.912 \\
.851 \\
.780 \\
.698 \\
.995\end{array}$ & & $\begin{array}{l}3.2,-1 \\
2.4,-1 \\
1.8,-1 \\
1.3,-1 \\
1.0,-1 \\
7.5,-2\end{array}$ & $\begin{array}{l}.443 \\
.322 \\
.221 \\
.134 \\
.082 \\
.043\end{array}$ \\
\hline & $\begin{array}{l}2.4,0 \\
1.8,0 \\
1.3,0 \\
1.0,0 \\
7.5,-1\end{array}$ & $\begin{array}{l}.805 \\
.758 \\
.705 \\
.659 \\
.602\end{array}$ & .35 & $\begin{array}{l}1.8,0 \\
1.3,0 \\
1.0,0 \\
7.5,-1\end{array}$ & $\begin{array}{l}.972 \\
.922 \\
.855 \\
.759\end{array}$ & & $\begin{array}{l}1.0,0 \\
7.5,-1 \\
5.6,-1 \\
4.2,-1\end{array}$ & $\begin{array}{l}.635 \\
.567 \\
.497 \\
.424\end{array}$ & .45 & $\begin{array}{l}1.3,0 \\
1.0,0 \\
7.5,-1 \\
5.6,-1\end{array}$ & $\begin{array}{l}.983 \\
.953 \\
.889 \\
.788\end{array}$ \\
\hline .20 & $\begin{array}{l}\text { 5., } 6,0 \\
4.2,0 \\
3.2,0 \\
2.4,0 \\
1.8,0 \\
1.3,0\end{array}$ & $\begin{array}{l}.902 \\
.984 \\
.959 \\
.821 \\
.800 \\
.725\end{array}$ & & $\begin{array}{l}5.6,-1 \\
4.2,-1 \\
3.2,-1 \\
2.4,-1 \\
1.8,-1 \\
1.3,-1 \\
1.0,-1\end{array}$ & $\begin{array}{l}.646 \\
.532 \\
.432 \\
.337 \\
.253 \\
.172 \\
.117\end{array}$ & .25 & $\begin{array}{l}4.2,0 \\
3.2,0 \\
2.4,0 \\
1.8,0 \\
1.3,0 \\
1.0,0 \\
7.5,-1\end{array}$ & $\begin{array}{l}.989 \\
.967 \\
.924 \\
.858 \\
.763 \\
.679 \\
.590\end{array}$ & & $\begin{array}{l}\text { 4. } 2,-1 \\
3.2,-1 \\
2.4,-1 \\
1.8,-1 \\
1.3,-1 \\
1.0,-1 \\
7.5,-2\end{array}$ & $\begin{array}{l}.661 \\
.527 \\
.387 \\
.263 \\
.153 \\
.090 \\
.045\end{array}$ \\
\hline
\end{tabular}




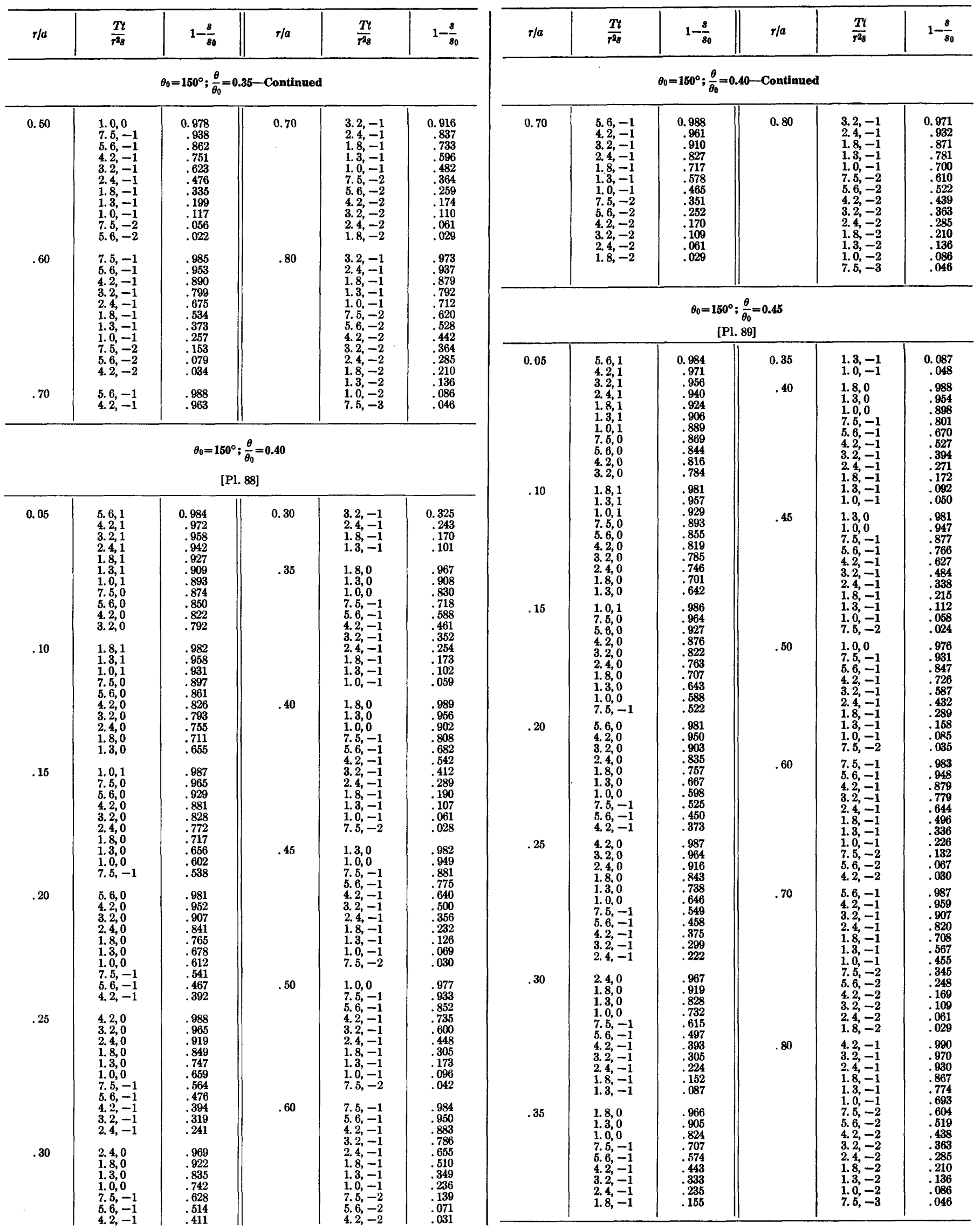


HYDRAULIC DIFFUSIVITY, WEDGE-SHAPED AQUIFERS DRAINED BY STREAMS

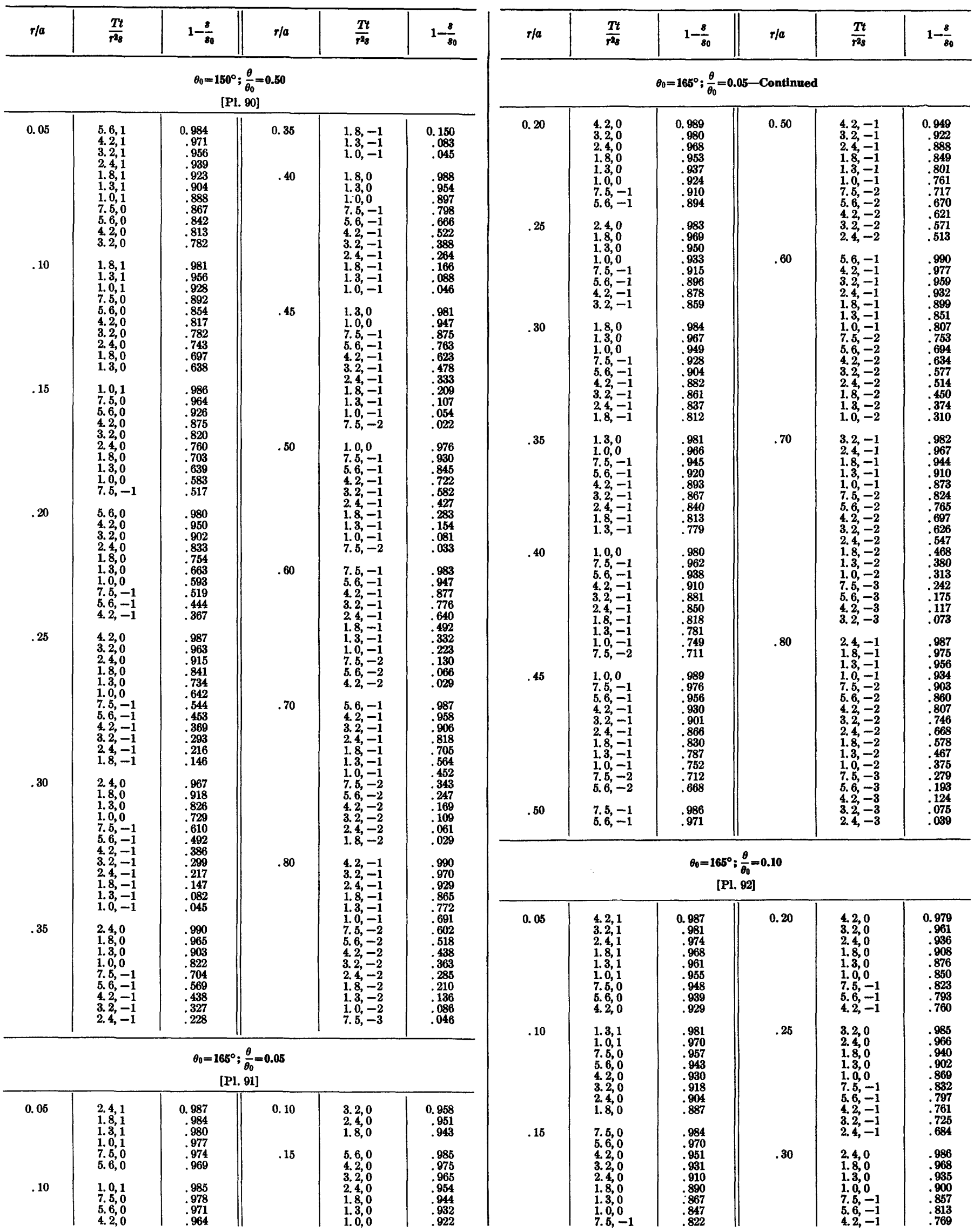




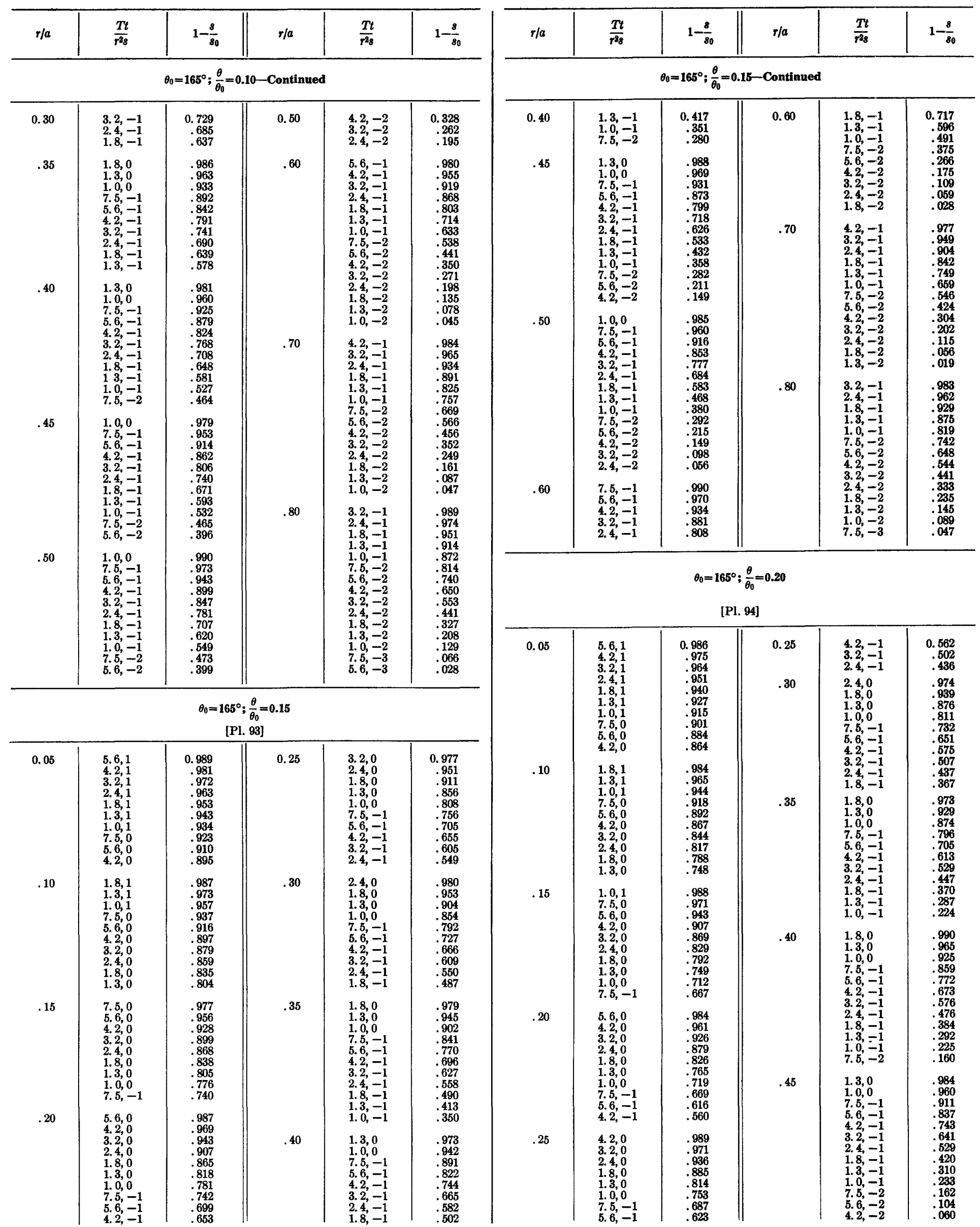


HYDRAULIC DIFFUSIVITY, WEDGE-SHAPED AQUIFERS DRAINED BY STREAMS

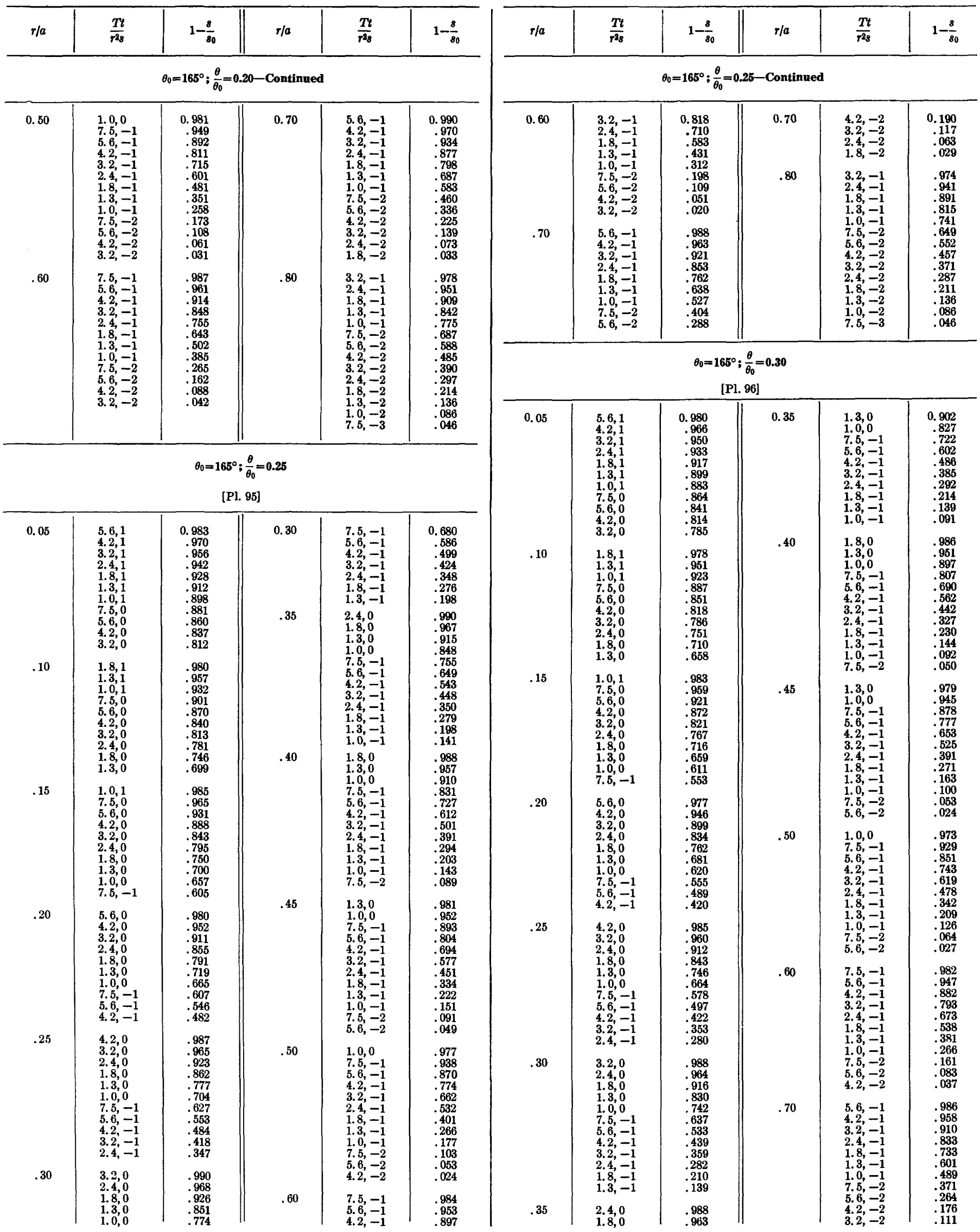




\begin{tabular}{|c|c|c|c|c|c|}
\hline$r / a$ & $\frac{T t}{T^{28}}$ & $1-\frac{s}{80}$ & $r / a$ & $\frac{T t}{T^{2} 8}$ & $1-\frac{8}{80}$ \\
\hline
\end{tabular}

\begin{tabular}{l|l|l||l|l|l}
\hline 0.70 & $2.4,-2$ & 0.061 & 0.80 & $7.5,-2$ & 0.625 \\
& $1.8,-2$ & .029 & & $5.6,-2$ & .532 \\
.80 & $4.2,-1$ & .989 & & $4.2,-2$ & .444 \\
& $3.2,-1$ & .970 & & $3.2,-2$ & .365 \\
& $2.4,-1$ & .933 & & $2.4,-2$ & .285 \\
& $1.8,-1$ & .877 & & $1.8,-2$ & .210 \\
& $1.3,-1$ & .794 & & $1.3,-2$ & .136 \\
& $1.0,-1$ & .716 & & $1.0,-2$ & .086 \\
& & & & \\
\hline
\end{tabular}

$\theta_{0}=165^{\circ} ; \frac{\theta}{\theta_{0}}=\mathbf{0 . 3 5}$

[P1. 97]

\begin{tabular}{|c|c|c|c|c|c|}
\hline \multirow[t]{2}{*}{0.05} & $\begin{array}{l}7.5,1 \\
5.6,1 \\
4.2,1\end{array}$ & $\begin{array}{r}0.990 \\
.978 \\
.962\end{array}$ & 0.35 & $\begin{array}{l}1.8,-1 \\
1.3,-1 \\
1.0,-1\end{array}$ & $\begin{array}{r}0.169 \\
.101 \\
.061\end{array}$ \\
\hline & $\begin{array}{l}3.2,1 \\
2.4,1 \\
1.8,1 \\
1.3,1 \\
1.0,1 \\
7.5,0 \\
5.6,0 \\
4.2,0 \\
3.2,0\end{array}$ & $\begin{array}{l}.945 \\
.927 \\
.909 \\
.889 \\
.872 \\
.850 \\
.825 \\
.796 \\
.764\end{array}$ & .40 & $\begin{array}{l}1.8,0 \\
1.3,0 \\
1.0,0 \\
7.5-1 \\
5.6,-1 \\
4.2,-1 \\
3.2,-1 \\
2.4,-1 \\
1.8,-1\end{array}$ & $\begin{array}{l}.985 \\
.946 \\
.886 \\
.788 \\
.661 \\
.524 \\
.398 \\
.281 \\
.185\end{array}$ \\
\hline \multirow[t]{2}{*}{.10} & $\begin{array}{l}1.8,1 \\
1.3,1 \\
1.0 .1\end{array}$ & $\begin{array}{l}.975 \\
.946 \\
.915\end{array}$ & & $\begin{array}{l}1.3,-1 \\
1.0,-1 \\
7.5,-2\end{array}$ & $\begin{array}{l}.106 \\
.062 \\
.030\end{array}$ \\
\hline & 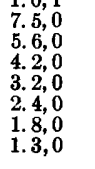 & $\begin{array}{l}.876 \\
.836 \\
.799 \\
.765 \\
.726 \\
.682 \\
.626\end{array}$ & .45 & $\begin{array}{l}1.3,0 \\
1.0,0 \\
7.5,-1 \\
5.6,-1 \\
4.2,-1 \\
3.2,-1 \\
2.4,-1\end{array}$ & $\begin{array}{l}.976 \\
.939 \\
.866 \\
.756 \\
.622 \\
.485 \\
.347 \\
228\end{array}$ \\
\hline \multirow[t]{2}{*}{.15} & $\begin{array}{l}1.0,1 \\
7.5,0 \\
5.6,0\end{array}$ & $\begin{array}{l}.981 \\
.955 \\
.913 \\
.959\end{array}$ & & $\begin{array}{l}1.8,-1 \\
1.3,-1 \\
1.0,-1 \\
7.5,-2\end{array}$ & $\begin{array}{l}.228 \\
.126 \\
.070 \\
.032\end{array}$ \\
\hline & $\begin{array}{l}4.2,0 \\
3.2,0 \\
2.4,0 \\
1.8,0 \\
1.3,0 \\
1.0,0 \\
7.5,-1\end{array}$ & $\begin{array}{l}.859 \\
.803 \\
.744 \\
.688 \\
.627 \\
.575 \\
.513\end{array}$ & .50 & $\begin{array}{l}1.0,0 \\
7.5,-1 \\
5.6,-1 \\
4.2,-1 \\
3.2,-1 \\
2.4,-1\end{array}$ & $\begin{array}{l}.971 \\
.922 \\
.837 \\
.718 \\
.586 \\
.439 \\
.301\end{array}$ \\
\hline \multirow[t]{2}{*}{.20} & $\begin{array}{l}\text { 5. } 6,0 \\
4.2,0 \\
3.2,0 \\
2.0\end{array}$ & $\begin{array}{l}.975 \\
.940 \\
.888 \\
.818\end{array}$ & & $\begin{array}{l}1.8,-1 \\
1.3,-1 \\
1.0,-1 \\
7.5,-2\end{array}$ & $\begin{array}{l}.001 \\
.172 \\
.097 \\
.044\end{array}$ \\
\hline & $\begin{array}{l}2.4,0 \\
1.8,0 \\
1.3,0 \\
1.0,0 \\
7.5,-1 \\
5.6,-1 \\
4.2,-1\end{array}$ & $\begin{array}{l}.818 \\
.738 \\
.650 \\
.584 \\
.515 \\
.445 \\
.374\end{array}$ & .60 & $\begin{array}{l}7.5,-1 \\
5.6,-1 \\
4.2,-1 \\
3.2,-1 \\
2.4,-1 \\
1.8,-1\end{array}$ & $\begin{array}{l}.980 \\
.941 \\
.871 \\
.773 \\
.645 \\
.504 \\
348\end{array}$ \\
\hline \multirow[t]{2}{*}{.25} & $\begin{array}{l}4.2,0 \\
3.2,0 \\
2.4,0 \\
1.8,0\end{array}$ & $\begin{array}{l}.983 \\
.956 \\
.903 \\
.827\end{array}$ & & $\begin{array}{l}1.0,-1 \\
1.0,-1 \\
7.5,-2 \\
5.6,-2 \\
4.2,-2\end{array}$ & $\begin{array}{l}.348 \\
.237 \\
.141 \\
.072 \\
.031\end{array}$ \\
\hline & $\begin{array}{l}1.3,0 \\
1.0,0 \\
7.5,-1 \\
5.6,-1 \\
4.2,-1 \\
3.2,-1 \\
2.4,-1\end{array}$ & $\begin{array}{l}.721 \\
.632 \\
.539 \\
.454 \\
.376 \\
.305 \\
.233\end{array}$ & .70 & $\begin{array}{l}5.6,-1 \\
4.2,-1 \\
3.2,-1 \\
2.4,-1 \\
1.8,-1 \\
1.3,-1\end{array}$ & $\begin{array}{l}.984 \\
.954 \\
.901 \\
.818 \\
.711 \\
.575\end{array}$ \\
\hline \multirow[t]{2}{*}{.30} & $\begin{array}{l}3.2,0 \\
2.4,0 \\
1.8,0 \\
1.3,0 \\
1.0,0 \\
7.5,-1\end{array}$ & $\begin{array}{l}.987 \\
.960 \\
.907 \\
.813 \\
.717 \\
.603 \\
492\end{array}$ & & $\begin{array}{l}7.5,-2 \\
5.6,-2 \\
4.2,-2 \\
3.2,-2 \\
2.4,-2 \\
1.8,-2\end{array}$ & $\begin{array}{l}.400 \\
.353 \\
.253 \\
.171 \\
.109 \\
.061 \\
.029\end{array}$ \\
\hline & $\begin{array}{l}\text { 5. } 6,-1 \\
\text { 4. } 2,-1 \\
\text { 3. } 2,-1 \\
\text { 2. } 4,-1 \\
\text { 1. } 8,-1 \\
\text { 1. } 3,-1\end{array}$ & $\begin{array}{l}.492 \\
.393 \\
.311 \\
.234 \\
.166 \\
.101\end{array}$ & 80 & $\begin{array}{l}\text { 4. } 2,-1 \\
3.2,-1 \\
2.4,-1 \\
1.8,-1 \\
1.3,-1\end{array}$ & $\begin{array}{l}.988 \\
.967 \\
.926 \\
.866 \\
.778 \\
699\end{array}$ \\
\hline .35 & $\begin{array}{l}2.4,0 \\
1.8,0 \\
1.3,0 \\
1.0,0 \\
7.5,-1 \\
5.6,-1 \\
4.2,-1 \\
3.2,-1 \\
2.4,-1\end{array}$ & $\begin{array}{l}.987 \\
.959 \\
.893 \\
.810 \\
.695 \\
.566 \\
.443 \\
.338 \\
.245\end{array}$ & & $\begin{array}{l}1.0,-1 \\
7.5,=2 \\
5.6,-2 \\
4.2,-2 \\
3.2,-2 \\
2.4,-2 \\
1.8,-2 \\
1.3,-2 \\
1.0,-2 \\
7.5,-3\end{array}$ & $\begin{array}{l}.699 \\
.611 \\
.523 \\
.440 \\
.363 \\
.285 \\
.210 \\
.136 \\
.086 \\
.046\end{array}$ \\
\hline
\end{tabular}

\begin{tabular}{c|c|c||c|c|c}
\hline$r / a$ & $\frac{T t}{r^{2}}$ & $1-\frac{8}{8_{0}}$ & $r / a$ & $\frac{T t}{r^{2}}$ & $1-\frac{8}{8_{0}}$ \\
\hline
\end{tabular}

[P1. 98]

\begin{tabular}{|c|c|c|c|c|c|}
\hline \multirow[t]{2}{*}{0.05} & $\begin{array}{l}7.5,1 \\
5.6,1 \\
4.2,1 \\
3.2,1\end{array}$ & $\begin{array}{r}0.989 \\
.977 \\
.960 \\
.941\end{array}$ & 0.35 & $\begin{array}{l}2.4,-1 \\
1.8,-1 \\
1.3,-1 \\
1.0,--1\end{array}$ & $\begin{array}{l}0.214 \\
.141 \\
.079 \\
.044\end{array}$ \\
\hline & $\begin{array}{l}2.4,1 \\
1.8,1 \\
1.3,1 \\
1.0,1 \\
7.5,0 \\
5.6,0 \\
4.2,0 \\
3.2,0\end{array}$ & $\begin{array}{l}.922 \\
.903 \\
.881 \\
.863 \\
.840 \\
.813 \\
.782 \\
.749\end{array}$ & .40 & $\begin{array}{l}1.8,0 \\
1.3,0 \\
1.0,0 \\
7.5,-1 \\
5.6,-1 \\
4.2,-1 \\
3.2,-1 \\
2.4,-1\end{array}$ & $\begin{array}{l}.984 \\
.943 \\
.879 \\
.774 \\
.640 \\
.497 \\
.368 \\
.250\end{array}$ \\
\hline \multirow[t]{2}{*}{.10} & $\begin{array}{l}2.4,1 \\
1.8,1\end{array}$ & $\begin{array}{r}.990 \\
.974\end{array}$ & & $\begin{array}{l}1.8,-1 \\
1.3,-1 \\
1.0,-1\end{array}$ & $\begin{array}{l}.157 \\
.084 \\
.045\end{array}$ \\
\hline & $\begin{array}{l}1.3,1 \\
1.0,1 \\
7.5,0 \\
5.6,0 \\
4.2,0 \\
3.2,0 \\
2.4,0 \\
1.8,0 \\
1.3,0\end{array}$ & $\begin{array}{l}.943 \\
.909 \\
.868 \\
.826 \\
.786 \\
.750 \\
.708 \\
.662 \\
.603\end{array}$ & .45 & $\begin{array}{l}1.3,0 \\
1.0,0 \\
7.5,-1 \\
5.6,-1 \\
4.2,-1 \\
3.2,-1 \\
2.4,-1 \\
1.8,-1\end{array}$ & $\begin{array}{l}.975 \\
.935 \\
.857 \\
.740 \\
.599 \\
.458 \\
.318 \\
.200 \\
104\end{array}$ \\
\hline \multirow[t]{2}{*}{.15} & $\begin{array}{l}1.0,1 \\
7.5,0\end{array}$ & $\begin{array}{r}.980 \\
.952 \\
.907\end{array}$ & & $\begin{array}{l}1.0,--1 \\
7.5,-2\end{array}$ & .053 \\
\hline & $\begin{array}{l}5.6,0 \\
4.2,0 \\
3.2,0 \\
2.4,0 \\
1.8,0 \\
1.3,0 \\
1.0,0 \\
7.5,-1\end{array}$ & $\begin{array}{l}.807 \\
.890 \\
.727 \\
.668 \\
.604 \\
.549 \\
.485\end{array}$ & .50 & $\begin{array}{l}1.0,0 \\
7.5,-1 \\
5.6,-1 \\
4.2,-1 \\
3.2,-1 \\
2.4,-1 \\
1.8,-1 \\
1.3,-1\end{array}$ & $\begin{array}{l}.969 \\
.917 \\
.826 \\
.701 \\
.562 \\
.412 \\
.274 \\
.150\end{array}$ \\
\hline \multirow[t]{2}{*}{.20} & $\begin{array}{l}5.6,0 \\
4.2,0\end{array}$ & $\begin{array}{l}.973 \\
.936\end{array}$ & & $\begin{array}{l}1.0,-1 \\
7.5,--2\end{array}$ & $\begin{array}{l}.080 \\
.034\end{array}$ \\
\hline & $\begin{array}{l}3.2,0 \\
2.4,0 \\
1.8,0 \\
1.3,0 \\
1.0,0 \\
\mathbf{7 . 5},-1 \\
\mathbf{5 . 6 ,}=1 \\
4.2,-1\end{array}$ & $\begin{array}{l}.881 \\
.805 \\
.721 \\
.628 \\
.559 \\
.487 \\
.414 \\
.341\end{array}$ & .60 & $\begin{array}{l}7.5,-1 \\
5.6,-1 \\
4.2,-1 \\
3.2,-1 \\
2.4,-1 \\
1.8,-1 \\
1.3,-1 \\
1.0,-1\end{array}$ & $\begin{array}{l}.978 \\
.937 \\
.862 \\
.759 \\
.626 \\
.482 \\
.328 \\
.221\end{array}$ \\
\hline \multirow[t]{2}{*}{.25} & $\begin{array}{l}4.2,0 \\
3.2,0 \\
2.4,0\end{array}$ & $\begin{array}{l}.982 \\
.953 \\
.897\end{array}$ & & $\begin{array}{l}7.5,-2 \\
5.6,-2 \\
4.2,-2\end{array}$ & $\begin{array}{l}.130 \\
.067 \\
.030\end{array}$ \\
\hline & $\begin{array}{l}1.8,0 \\
1.3,0 \\
1.0,0 \\
7.5,-1 \\
5.6,-1 \\
4.2,-1 \\
3.2,-1 \\
2.4,-1\end{array}$ & $\begin{array}{l}.815 \\
.703 \\
.609 \\
.512 \\
.423 \\
.344 \\
.272 \\
.201\end{array}$ & .70 & $\begin{array}{l}\text { 5. } 6,-1 \\
4.2,-1 \\
3.2,-1 \\
2.4,-1 \\
1.8,-1 \\
1.3,-1 \\
1.0,-1\end{array}$ & $\begin{array}{l}.983 \\
.951 \\
.895 \\
.806 \\
.696 \\
.559 \\
.451 \\
.343\end{array}$ \\
\hline \multirow[t]{2}{*}{.30} & $\begin{array}{l}3.2,0 \\
2.4,0 \\
1.8,0 \\
1.3,0 \\
1.0,0\end{array}$ & $\begin{array}{l}.986 \\
.957 \\
.901 \\
.801 \\
.699\end{array}$ & & $\begin{array}{l}5.6,-2 \\
4.2,-2 \\
3.2,-2 \\
2.4,-2 \\
1.8,-2\end{array}$ & $\begin{array}{l}.248 \\
.169 \\
.109 \\
.061 \\
.029\end{array}$ \\
\hline & $\begin{array}{l}7.5,-1 \\
5.6,-1 \\
4.2,-1 \\
3.2,-1 \\
2.4,-1 \\
1.8,-1 \\
1.3,-1\end{array}$ & $\begin{array}{l}.579 \\
.463 \\
.361 \\
.279 \\
.203 \\
.138 \\
.079\end{array}$ & .80 & $\begin{array}{l}4.2,-1 \\
3.2,-1 \\
2.4,-1 \\
1.8,-1 \\
1.3,-1 \\
1.0,-1\end{array}$ & $\begin{array}{l}.987 \\
.965 \\
.922 \\
.858 \\
.767 \\
.688 \\
.602\end{array}$ \\
\hline .35 & $\begin{array}{l}2.4,0 \\
1.8,0 \\
1.3,0 \\
1.0,0 \\
7.5,-1 \\
5.6,-1 \\
4.2,-1 \\
3.2,-1\end{array}$ & $\begin{array}{l}.986 \\
.956 \\
.885 \\
.797 \\
.675 \\
.540 \\
.413 \\
.306\end{array}$ & & $\begin{array}{l}5.6,-2 \\
4.2,-2 \\
3.2,-2 \\
2.4,-2 \\
1.8,-2 \\
1.3,-2 \\
1.0,-2 \\
7.5,-3\end{array}$ & $\begin{array}{l}.518 \\
.438 \\
.363 \\
.285 \\
.210 \\
.136 \\
.086 \\
.046\end{array}$ \\
\hline
\end{tabular}

$\theta_{0}=165^{\circ} ; \frac{\theta}{\theta_{0}}=0.45$

[Pl. 99]

\begin{tabular}{l|r|r||r|r|r}
\hline 0.05 & $7.5,1$ & 0.988 & 0.05 & $5.6,0$ & 0.806 \\
& $5.6,1$ & .976 & & .774 \\
& $4.2,1$ & .958 & & $3.2,0$ & .739 \\
& $3.2,1$ & .939 & & & \\
& $2.4,1$ & .919 & .10 & $2.4,1$ & .989 \\
& $1.8,1$ & .899 & & $1.8,1$ & .973 \\
& $1.3,1$ & .877 & & $1.3,1$ & .940 \\
& $1.0,1$ & .858 & & $1.0,1$ & .906 \\
& $7.5,0$ & & & & \\
& & & &
\end{tabular}



HYDRAULIC DIFFUSIVITY, WEDGE-SHAPED AQUIFERS DRAINED BY STREAMS

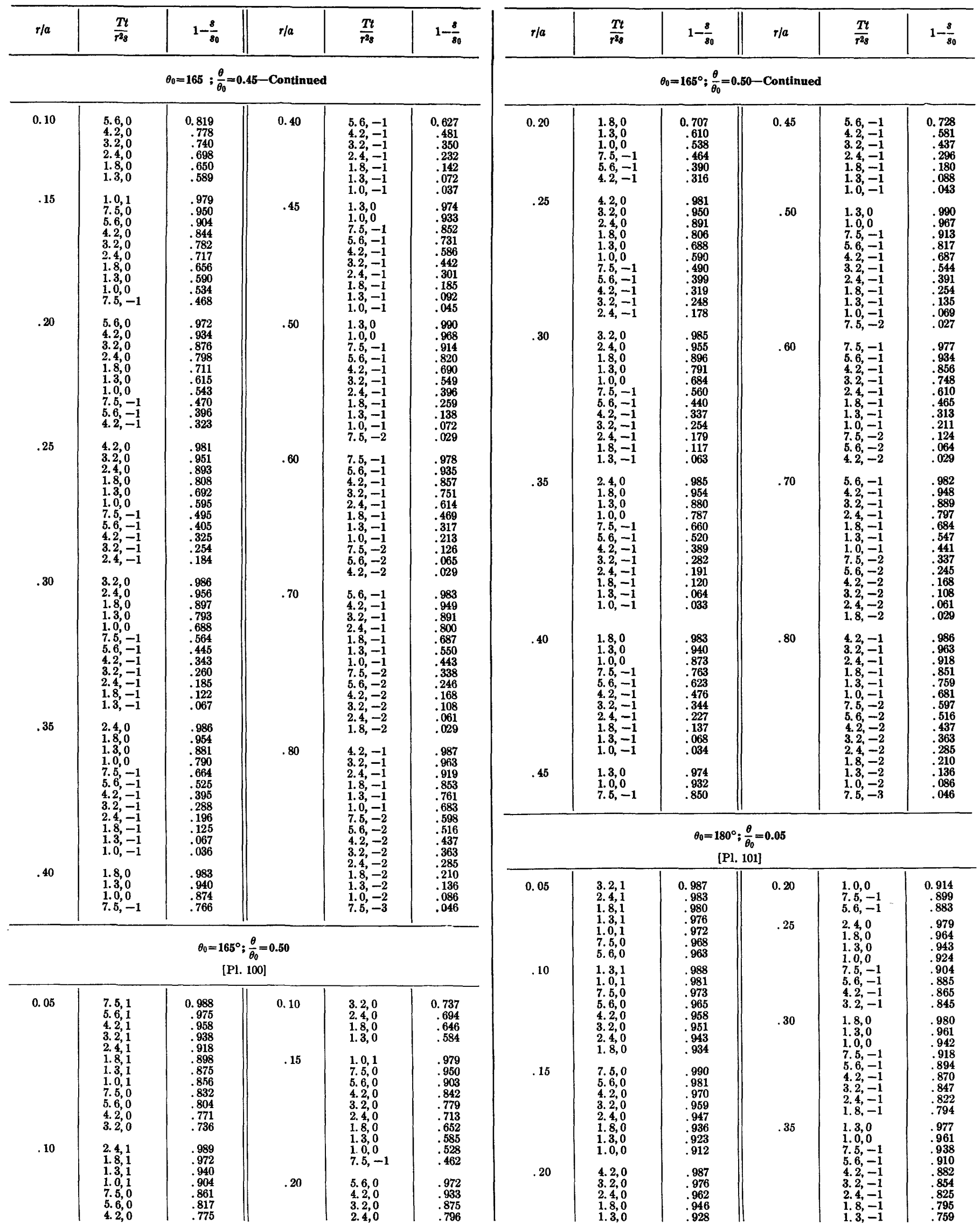




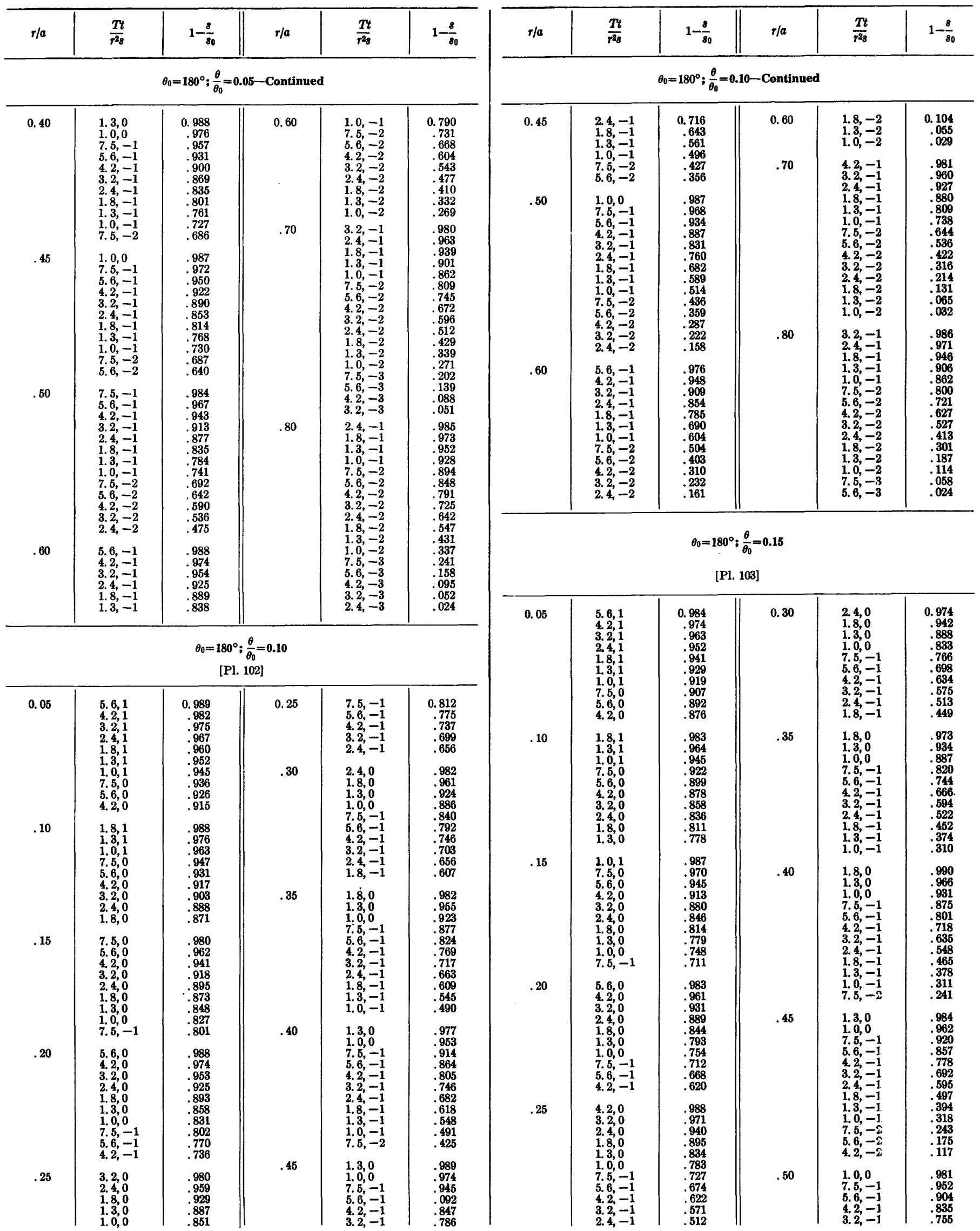


HYDRAULIC DIFFUSIVITY, WEDGE-SHAPED AQUIFERS DRAINED BY STREAMS

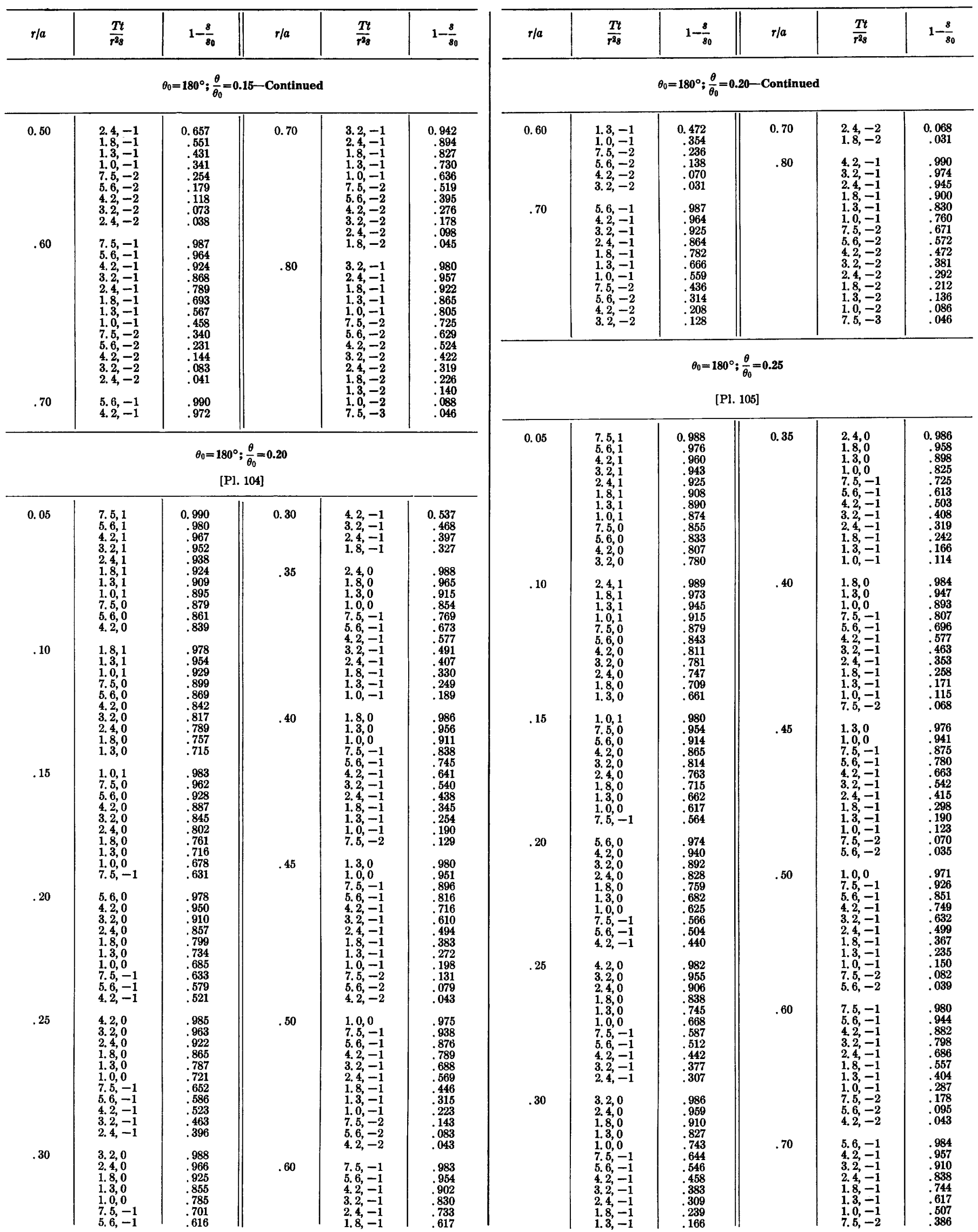




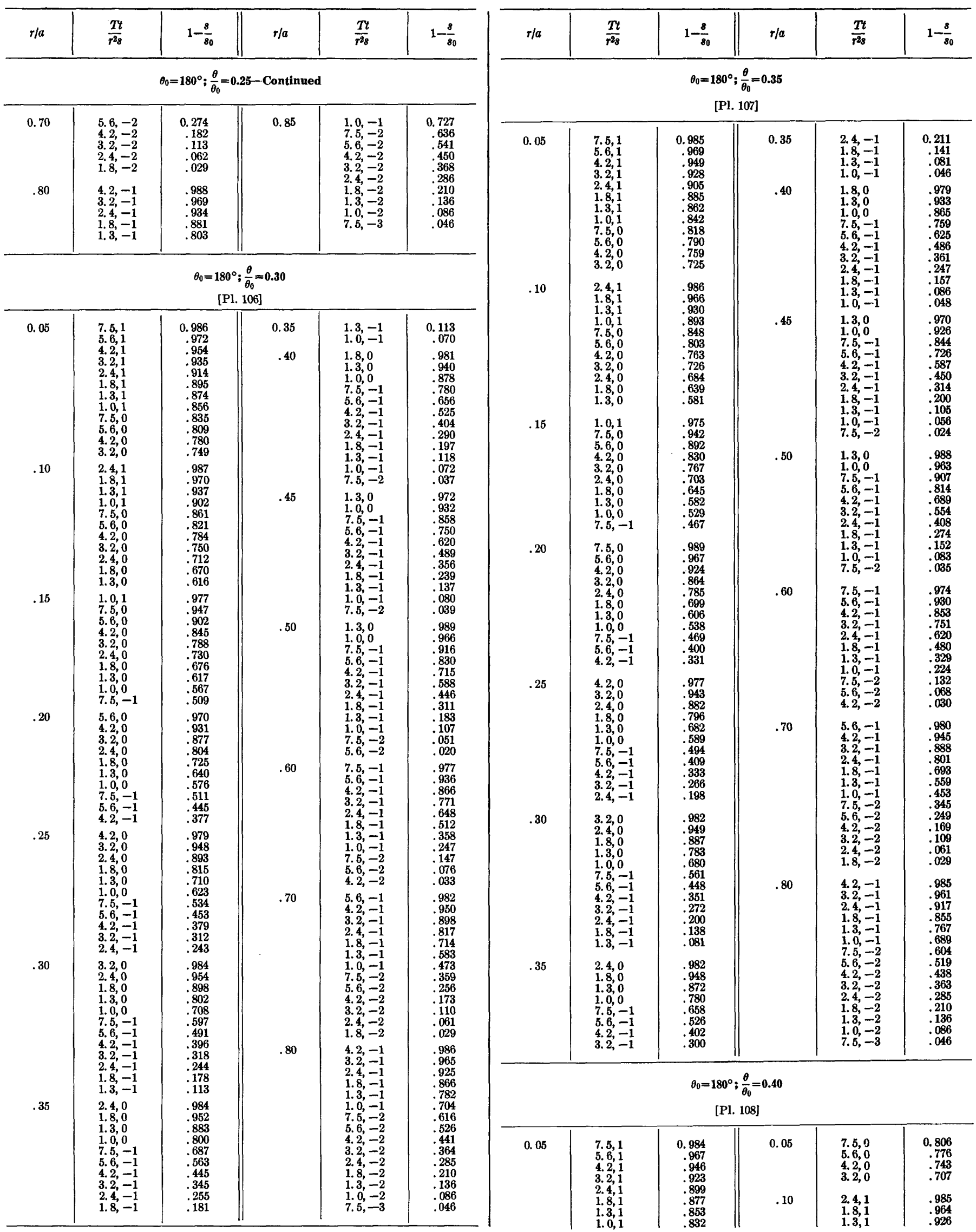




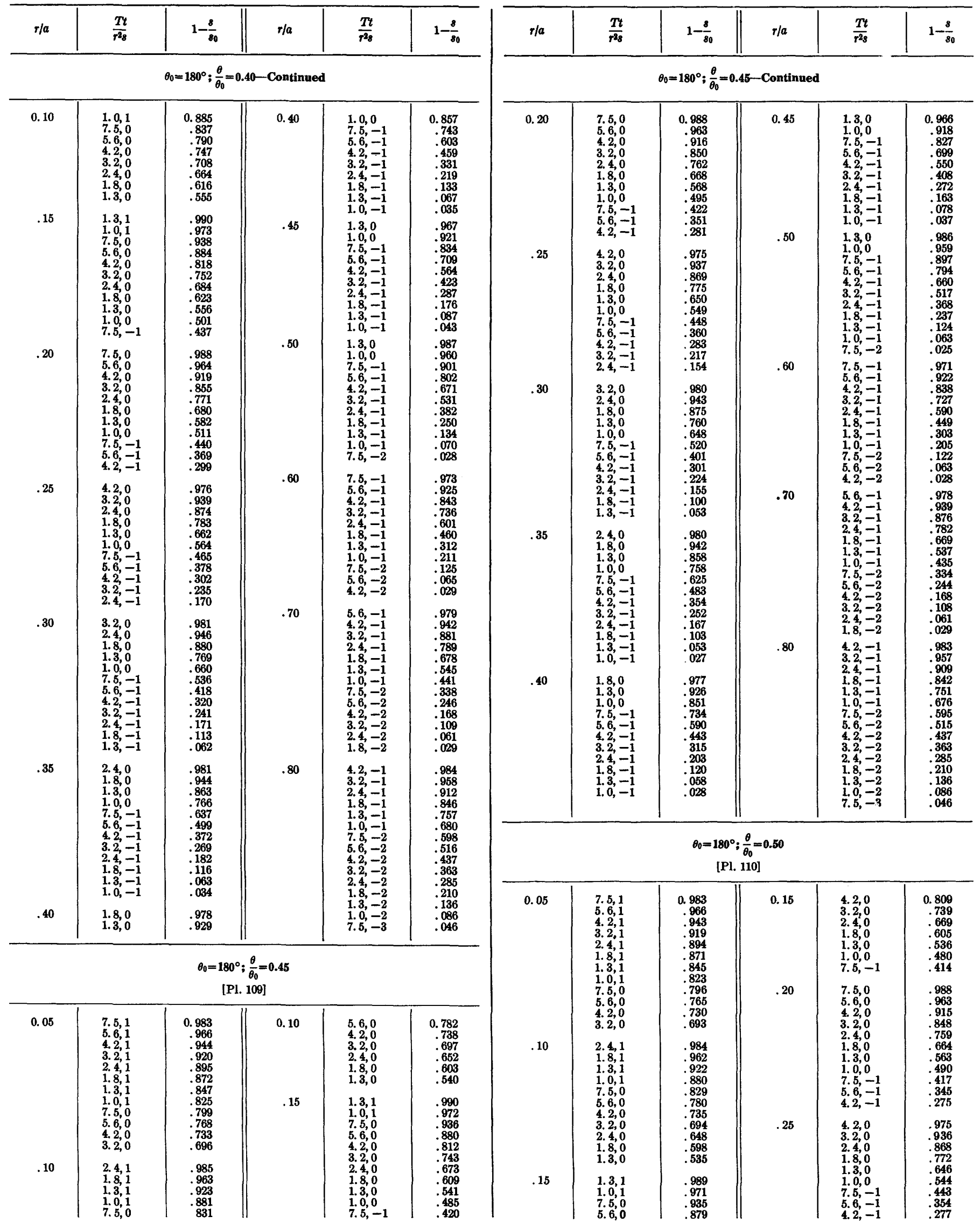


TABLES

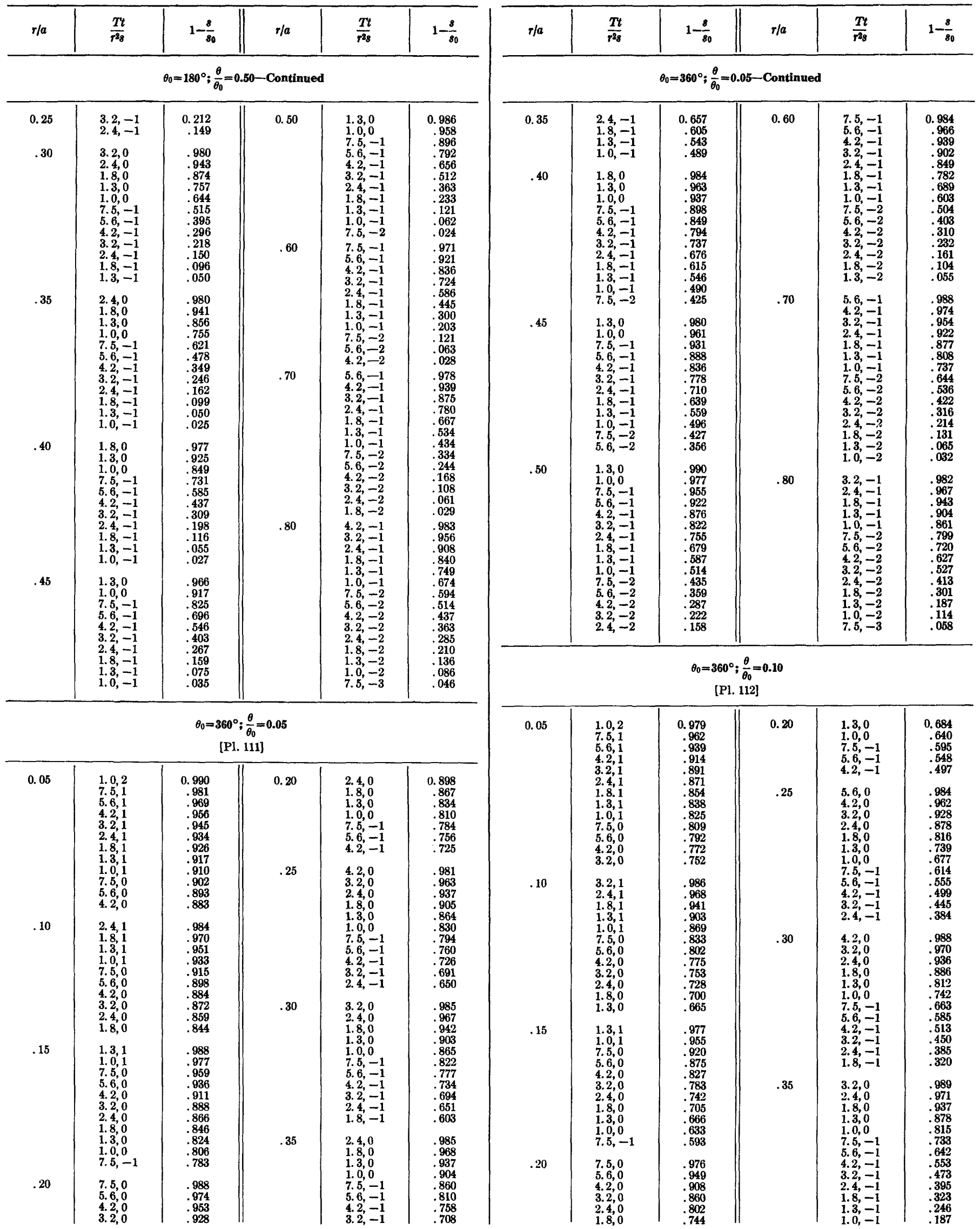




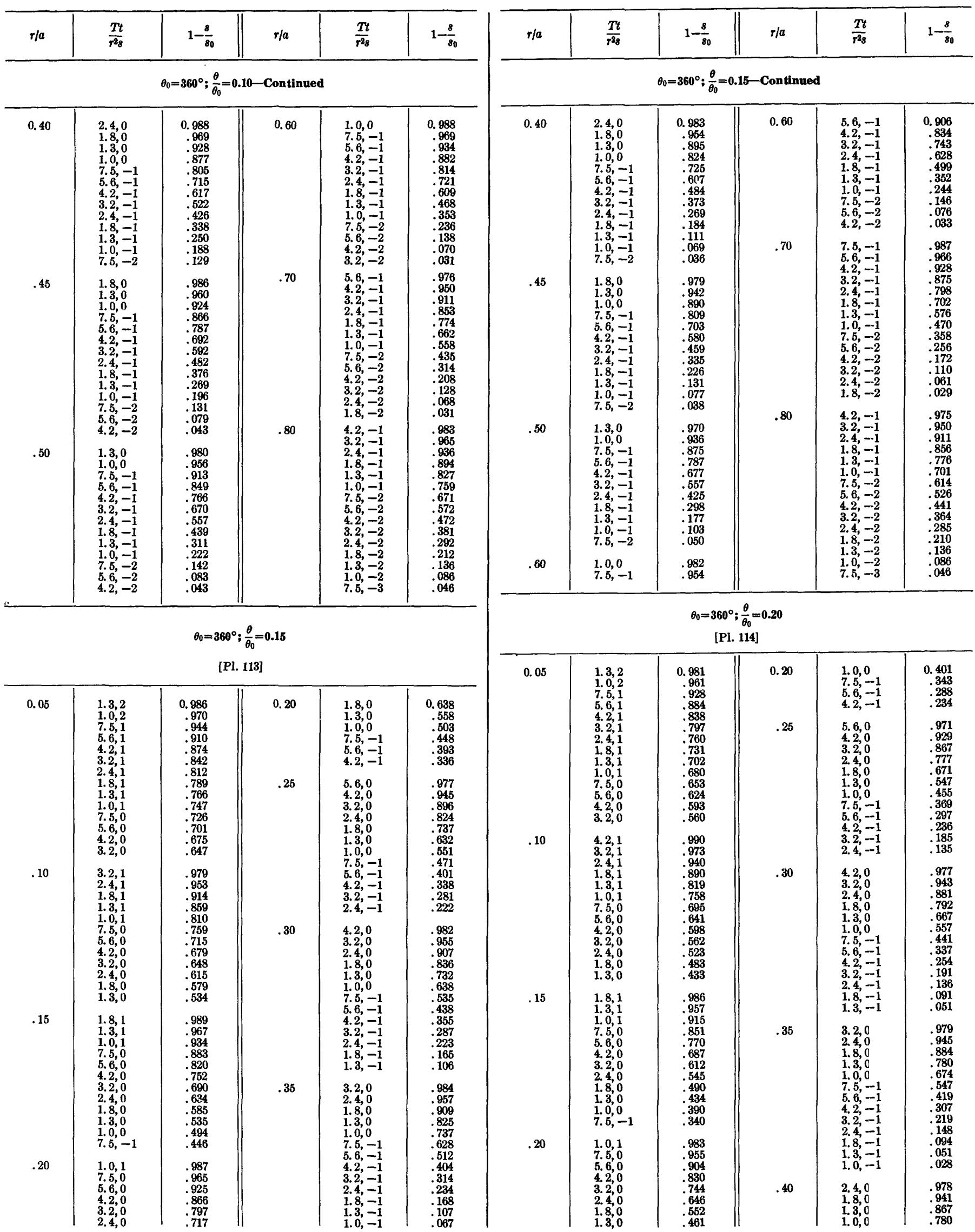


TABLES

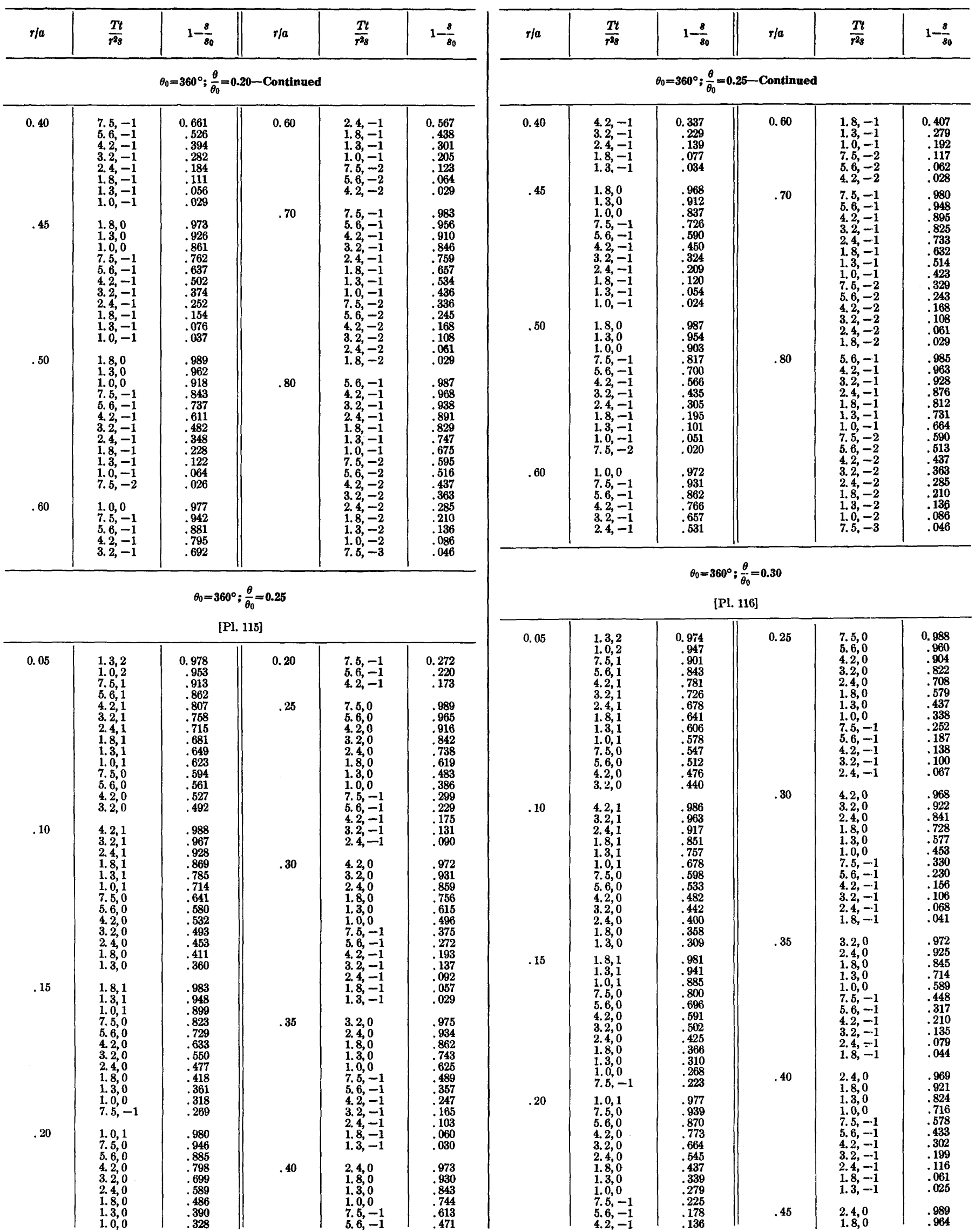


HYDRAULIC DIFFUSIVITY, WEDGE-SHAPED AQUIFERS DRAINED BY STREAMS

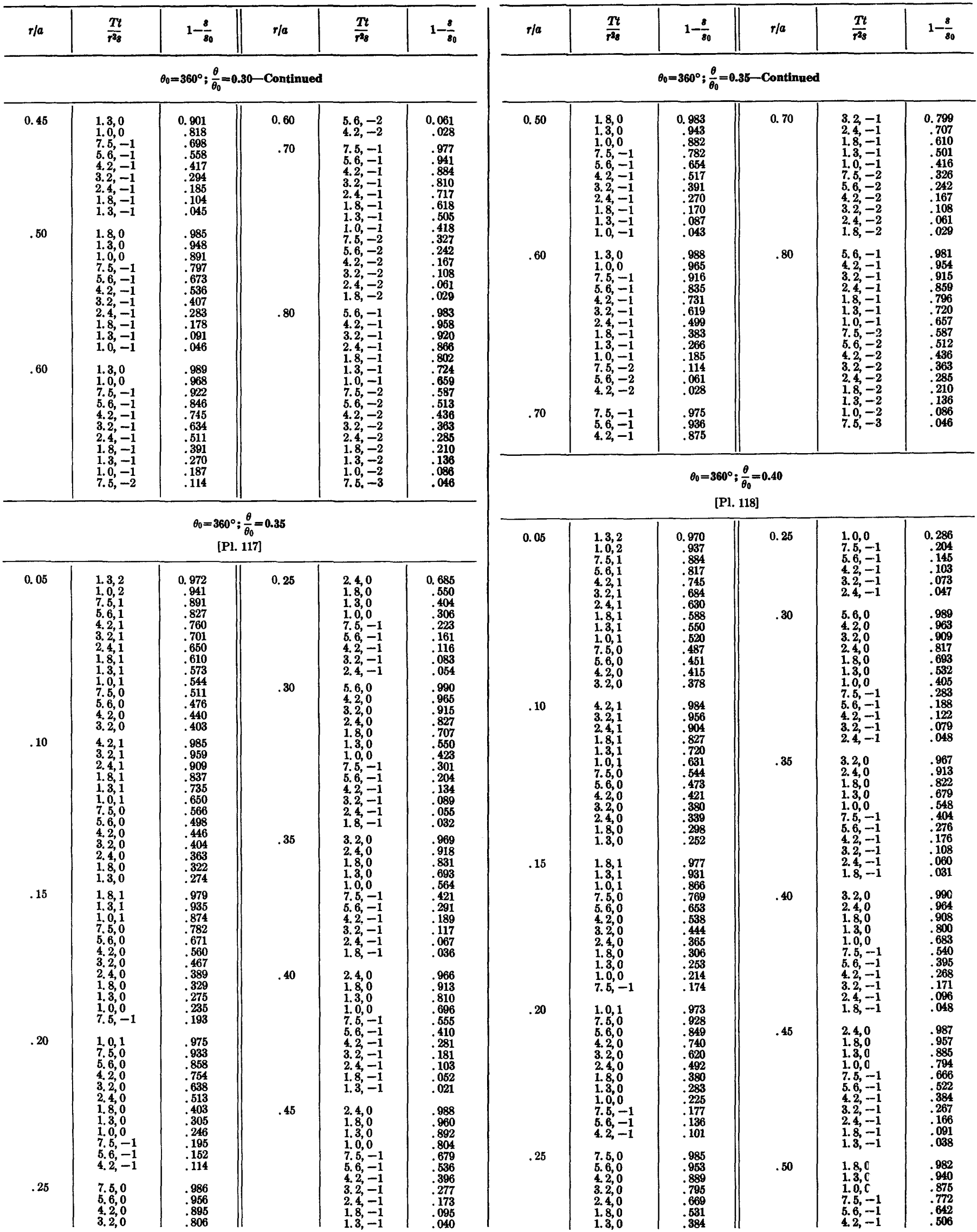




\begin{tabular}{|c|c|c|c|c|c|c|c|c|c|c|c|}
\hline$r / a$ & $\frac{T t}{r^{2}}$ & $1-\frac{8}{80}$ & $r / a$ & $\frac{T t}{r^{2}}$ & $1-\frac{8}{80}$ & $r / a$ & $\frac{T t}{r_{28}}$ & $1-\frac{8}{80}$ & $r / a$ & $\frac{T_{t}}{r^{2}}$ & $1-\frac{8}{80}$ \\
\hline \multicolumn{6}{|c|}{$\theta_{0}=360^{\circ} ; \frac{\theta}{\theta_{0}}=0.40-$ Continued } & \multicolumn{6}{|c|}{$\theta_{0}=360^{\circ} ; \frac{\theta}{\theta_{0}}=0.45-$ Continued } \\
\hline 0.50 & $\begin{array}{l}3.2,-1 \\
2.4,-1 \\
1.8,-1 \\
1.3,-1 \\
1.0,-1\end{array}$ & $\begin{array}{r}0.381 \\
.263 \\
.1066 \\
.045\end{array}$ & 0.70 & $\begin{array}{l}1.3,-1 \\
1.0,1 \\
7.5,2 \\
5.6,2 \\
4.2,-2\end{array}$ & $\begin{array}{r}0.499 \\
.414 \\
.326 \\
.242 \\
.167\end{array}$ & \multirow[t]{3}{*}{0.60} & \multirow{3}{*}{$\begin{array}{l}1.3,0 \\
1.0,0 \\
7.5,-1 \\
5.6,-1 \\
4.2,-1 \\
3.2,-1 \\
2.4,-1 \\
1.8,-1 \\
1.3,-1 \\
1.0,-1 \\
7.5,-2 \\
5.6,-2 \\
4.2,-2\end{array}$} & \multirow{3}{*}{$\begin{array}{r}0.987 \\
.962 \\
.098 \\
.823 \\
.717 \\
.060 \\
.488 \\
.736 \\
.262 \\
.183 \\
.113 \\
.061 \\
.028\end{array}$} & 0.70 & 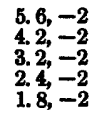 & $\begin{array}{l}0.242 \\
.167 \\
.108 \\
.061 \\
.029\end{array}$ \\
\hline \multirow[t]{3}{*}{.60} & $\begin{array}{l}1.3,0 \\
1.0,0\end{array}$ & $\begin{array}{l}.987 \\
.963\end{array}$ & & $\begin{array}{l}2.4,-2 \\
1.8,-2\end{array}$ & $\begin{array}{l}.061 \\
.029\end{array}$ & & & & .80 & $\begin{array}{l}\text { 5. } 6,-1 \\
4.2,-1 \\
3.2,-1\end{array}$ & 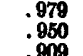 \\
\hline & $\begin{array}{l}\text { 5.6, } \\
4.2,-1 \\
3.2,-1 \\
2.4,-1\end{array}$ & $\begin{array}{l}.827 \\
.722 \\
.611 \\
.492\end{array}$ & .80 & $\begin{array}{l}\text { 5. } 6,-1 \\
4.2,-1 \\
3.2,-1 \\
2.4,-1\end{array}$ & $\begin{array}{l}.980 \\
.982 \\
.911 \\
.855\end{array}$ & & & & & $\begin{array}{l}2.4,-1 \\
\text { 1. } 8,-1 \\
1.3,-1 \\
\text { 1. } 0,-1\end{array}$ & $\begin{array}{l}.883 \\
.791 \\
.777 \\
.655 \\
5685\end{array}$ \\
\hline & $\begin{array}{l}1.8,-1 \\
1 ., 0,1 \\
7.5,-2 \\
5.6,2,2 \\
4.2,-2\end{array}$ & $\begin{array}{l}.263 \\
.184 \\
.113 \\
.061 \\
.028\end{array}$ & & $\begin{array}{l}1.8,-1 \\
1.3,1 \\
1.0,1 \\
7.6,2 \\
5.6,2 \\
4,2,-2\end{array}$ & $\begin{array}{l}.718 \\
.656 \\
.856 \\
.582 \\
.436\end{array}$ & \multirow[t]{2}{*}{.70} & $\begin{array}{l}7.5,-1 \\
5.6,-1 \\
4.2,-1 \\
3.2,-1 \\
2.4,-1 \\
1,-1\end{array}$ & $\begin{array}{l}.972 \\
.931 \\
.867 \\
.7898 \\
.698\end{array}$ & & 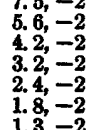 & 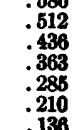 \\
\hline \multirow[t]{2}{*}{.70} & $\begin{array}{l}7.5,-1 \\
5.6,-1 \\
4.2,-1 \\
3.2,-1\end{array}$ & $\begin{array}{l}.973 \\
.933 \\
.870 \\
.793\end{array}$ & & $\begin{array}{l}2.4,-2 \\
1.8,2 \\
1.3,2-2 \\
1.0,-2\end{array}$ & $\begin{array}{l}.285 \\
.210 \\
.136\end{array}$ & & $\begin{array}{l}1.3,=1 \\
1.0,1 \\
7.6,-2\end{array}$ & $\begin{array}{l}.497 \\
.426 \\
.326\end{array}$ & & $\begin{array}{l}1.0,2 \\
7 ., 5,-3\end{array}$ & - 046 \\
\hline & $1.8,-1$ & .606 & & & & \multirow{2}{*}{\multicolumn{6}{|c|}{$\begin{array}{c}\theta_{0}=360^{\circ} ; \frac{\theta}{\theta_{0}}=0.50 \\
\text { [PI. 120] }\end{array}$}} \\
\hline \multicolumn{6}{|c|}{$\begin{array}{c}\theta_{0}=360^{\circ} ; \frac{\theta}{\theta_{0}}=0.45 \\
\text { [PI. 119] }\end{array}$} & & & & & & \\
\hline \multirow[t]{3}{*}{0.05} & $\begin{array}{l}1.3,2 \\
1.0,2 \\
7.5,1\end{array}$ & $\begin{array}{r}0.969 \\
.935 \\
.880\end{array}$ & 0.25 & $\begin{array}{l}4.2,-2, \\
3.2,1 \\
\text { 2. } 4,-1\end{array}$ & $\begin{array}{r}0.097 \\
.0088 \\
.044\end{array}$ & \multirow[t]{2}{*}{0.05} & \multirow{2}{*}{ 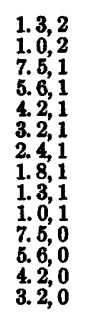 } & \multirow{2}{*}{ 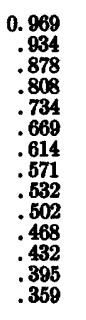 } & \multirow[t]{2}{*}{$\begin{array}{r}0.25 \\
.30\end{array}$} & \multirow{4}{*}{ 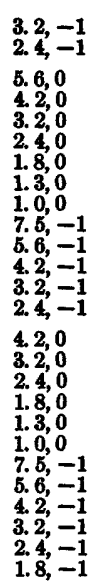 } & \multirow{4}{*}{ 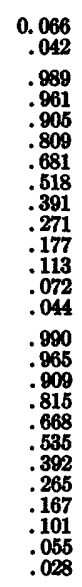 } \\
\hline & $\begin{array}{l}0.0,1 \\
4,2,1 \\
3.2,1 \\
2.4,1 \\
1.8,1 \\
1.3,1 \\
1.0,1 \\
7.5,0 \\
5,6,0 \\
4,2,0 \\
3,0\end{array}$ & $\begin{array}{l}.810 \\
.737 \\
.673 \\
.618 \\
.575 \\
.536 \\
.507 \\
.473 \\
.437 \\
.400\end{array}$ & .30 & 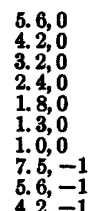 & $\begin{array}{l}.989 \\
.9801 \\
.806 \\
.811 \\
.684 \\
.5214 \\
.3944 \\
.2180 \\
.115\end{array}$ & & & & & & \\
\hline & $\frac{4,2,1}{2,1}$ & .983 & & $\begin{array}{l}3 . \overrightarrow{2},-1 \\
2.4,-1\end{array}$ & $\begin{array}{l}.074 \\
.045\end{array}$ & \multirow[t]{2}{*}{.10} & \multirow{2}{*}{$\begin{array}{l}4.2,1 \\
3.2,1,1 \\
2.4,1 \\
1.8,1 \\
1.3,1,1 \\
1.0,1 \\
7.6,0 \\
5.6,0 \\
4.2,0 \\
3.2,00 \\
2 ., 0 \\
1.8,00 \\
1.3,0\end{array}$} & \multirow{2}{*}{$\begin{array}{l}.983 \\
.985 \\
.899 \\
.818 \\
.707 \\
.616 \\
.546 \\
.450 \\
.302 \\
.320 \\
.221 \\
.235\end{array}$} & \multirow[t]{2}{*}{.35} & & \\
\hline .10 & $\begin{array}{l}2.4,1 \\
1.8,1 \\
1.3,1 \\
1.0,1 \\
7.5,0 \\
5,6,0 \\
4.2,0 \\
3.2,0 \\
2.4,0 \\
1,0.0\end{array}$ & $\begin{array}{l}.800 \\
.820 \\
.711 \\
.619 \\
.530 \\
.459 \\
.406 \\
.365 \\
.325 \\
.285\end{array}$ & .35 & $\begin{array}{l}\mathbf{4}, 2,0 \\
3.2,0 \\
2.4,0 \\
1.8,0 \\
1 ., 3,0 \\
1.0,0 \\
7.5,0 \\
5,6,-1 \\
4,2,1 \\
3.2,-1\end{array}$ & $\begin{array}{l}.990 \\
.996 \\
.910 \\
.816 \\
.671 \\
.538 \\
.395 \\
.2868 \\
.109\end{array}$ & & & & & & \\
\hline & $1.3,0$ & .239 & & $\begin{array}{l}2.4,-1 \\
1.8,-1\end{array}$ & $\begin{array}{l}.056 \\
.029\end{array}$ & \multirow[t]{3}{*}{.15} & \multirow{3}{*}{$\begin{array}{l}1.8,1 \\
1.3,1 \\
1.0,1 \\
7.5,0 \\
5.6,0 \\
4.2,0 \\
3.2,0 \\
2.4,0 \\
1.8,0 \\
1.3,0 \\
1.0,0 \\
7.5,-1\end{array}$} & \multirow{3}{*}{$\begin{array}{l}.976 \\
.928 \\
.860 \\
.7599 \\
.6592 \\
.5226 \\
.3477 \\
.2283 \\
.237 \\
.160\end{array}$} & .40 & \multirow{2}{*}{ 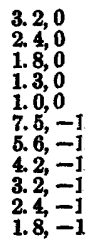 } & \multirow{2}{*}{ 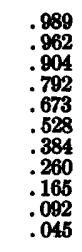 } \\
\hline \multirow{2}{*}{.15} & $\begin{array}{l}1.8,1 \\
1.3,1 \\
1.0,1 \\
7.5,0,0 \\
3.6,0 \\
4.2,0 \\
3.2,0 \\
2.4,0 \\
1 ., 4,0\end{array}$ & $\begin{array}{l}.976 \\
.928 \\
.862 \\
.761 \\
.642 \\
.526 \\
.350 \\
.293\end{array}$ & .40 & 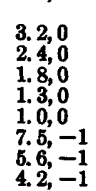 & 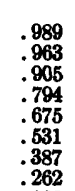 & & & & & & \\
\hline & $\begin{array}{l}1.3,0 \\
1.0,0 \\
7.5,-1\end{array}$ & $\begin{array}{l}.241 \\
.203 \\
.164\end{array}$ & & $\begin{array}{l}\text {. } 2,1 \\
\text { 2. } \\
1.8\end{array}$ & $\begin{array}{l}.060 \\
.003 \\
.045\end{array}$ & & & & .45 & $\begin{array}{l}2.4,0 \\
1.8,0 \\
1.3,0\end{array}$ & $\begin{array}{l}.986 \\
.985 \\
.880 \\
\end{array}$ \\
\hline .20 & $\begin{array}{l}1.0,1 \\
7.5,0 \\
5.6,0 \\
4.2,0 \\
3.2,0 \\
2.4,0 \\
1 ., 0,0 \\
1.3,0\end{array}$ & $\begin{array}{l}.972 \\
.926 \\
.844 \\
.732 \\
.609 \\
.480 \\
.367\end{array}$ & .45 & $\begin{array}{l}2.4,0 \\
1.8,0 \\
1.3,0 \\
1.0,0 \\
7.5,1 \\
5.6,0,1 \\
4.2,2 \\
3.2,-1\end{array}$ & $\begin{array}{l}.987 \\
.9856 \\
.8828 \\
.7859 \\
.515 \\
.3788 \\
.262\end{array}$ & \multirow{2}{*}{.20} & 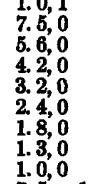 & $\begin{array}{l}.925 \\
: 825 \\
.729 \\
.8296 \\
.476 \\
.363 \\
.267 \\
.210\end{array}$ & & $\begin{array}{l}1.0,0 \\
7.0,0 \\
\text { S., },-1 \\
4 ., 2,-1 \\
3.2,-1 \\
2.4,-1 \\
1.8,-1 \\
1.3,-1\end{array}$ & 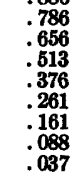 \\
\hline & $\begin{array}{l}1.0,0 \\
7.5,-1 \\
5.6,-1 \\
4.2-1\end{array}$ & $\begin{array}{l}.214 \\
.167 \\
.128\end{array}$ & & $\begin{array}{l}2.4,-1 \\
1.8,1 \\
1.3,-1\end{array}$ & $\begin{array}{l}.162 \\
.089 \\
.037\end{array}$ & & $\begin{array}{l}7.5,-1 \\
5.6,2 \\
4.2,-1\end{array}$ & $\begin{array}{l}: 124 \\
: 092 \\
.092\end{array}$ & .50 & $\begin{array}{l}1.8,0 \\
1.3,0 \\
1.0,0\end{array}$ & : 8882 \\
\hline .25 & $\begin{array}{l}7.5,0 \\
5,6,0 \\
4,2,0 \\
3.2,0 \\
2,4,0 \\
1.8,0 \\
1.3,0\end{array}$ & $\begin{array}{l}.985 \\
.951 \\
.885 \\
.789 \\
.660 \\
.620 \\
.372\end{array}$ & .50 & $\begin{array}{l}1.8,0 \\
1.3,0 \\
1,0,0 \\
7.5,0,1 \\
5,6,0,1 \\
4.2,1 \\
3.2,-1 \\
2.4,-1\end{array}$ & 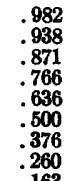 & .25 & $\begin{array}{l}7.5,0 \\
5.6,0 \\
4.2,00 \\
3.2,0 \\
2.4,00 \\
1.8,00 \\
1.3,00 \\
1.0,0\end{array}$ & $\begin{array}{l}.985 \\
.950 \\
.883 \\
.786 \\
.657 \\
.516 \\
.3688 \\
.271\end{array}$ & & $\begin{array}{l}7.5,-1 \\
5.6,-1 \\
4.2,2 \\
3.2,-1 \\
2.4,-1 \\
1.8,-1 \\
1.3,-1 \\
1.0,-1\end{array}$ & 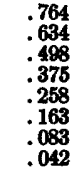 \\
\hline & $\begin{array}{l}7.5,01 \\
6,6,-1\end{array}$ & $\begin{array}{l}.280 \\
.137\end{array}$ & & $\begin{array}{l}1.3,2 \\
1.0,=1\end{array}$ & $\begin{array}{l}.000 \\
.042\end{array}$ & & $\begin{array}{l}5,6,-1 \\
4.2,-1\end{array}$ & $\begin{array}{l}0.134 \\
: 095\end{array}$ & .60 & $\begin{array}{l}1.3,0 \\
1.0,0\end{array}$ & . .987 \\
\hline
\end{tabular}


HYDRAULIC DIFFUSIVITY, WEDGE-SHAPED AQUIFERS DRAINED BY STREAMS

\begin{tabular}{|c|c|c|c|c|c|}
\hline$r / a$ & $\frac{T l}{r^{2}}$ & $1-\frac{8}{80}$ & $r / a$ & $\frac{T t}{r^{2} s}$ & $1-\frac{8}{80}$ \\
\hline \multicolumn{6}{|c|}{$\theta_{0}=360^{\circ} ; \frac{\theta}{\theta_{0}}=0.50-$ Continued } \\
\hline 0.60 & 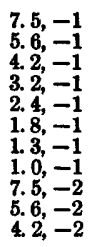 & $\begin{array}{r}0.907 \\
.822 \\
.715 \\
.605 \\
.487 \\
.376 \\
.262 \\
.183 \\
.113 \\
.061 \\
.028\end{array}$ & 0.70 & $\begin{array}{l}7.5,-1 \\
5.6,-1 \\
4 .,-1 \\
3.2,-1 \\
2.4,-1 \\
1.8,-1 \\
1.3,-1 \\
1.0,=1 \\
7.5,-2 \\
5.6,-2 \\
4.2,-2\end{array}$ & $\begin{array}{r}0.972 \\
.930 \\
.866 \\
.788 \\
.697 \\
.603 \\
.497 \\
.414 \\
.326 \\
.242 \\
.167\end{array}$ \\
\hline
\end{tabular}

\begin{tabular}{c|c|c|c|c|c|c}
\hline$r / a$ & $\frac{T t}{r^{2}}$ & $1-\frac{8}{80}$ & $r / a$ & $\frac{T t}{r^{28}}$ & $1-\frac{8}{80}$ \\
$\theta_{0}=360^{\circ} ; \frac{\theta}{\theta_{0}}=0.50-$ Continued
\end{tabular}

\begin{tabular}{l|l|l||l|l|l}
\hline 0.70 & $3.2,-2$ & 0.108 & 0.80 & $1.0,-1$ & 0.655 \\
& $2.4,-2$ & .061 & & $7.5,-2$ & .586 \\
& $1.8,-2$ & .029 & & $5.6,-2$ & .512 \\
.80 & $5.6,-1$ & .979 & & $42,-2$ & .436 \\
& $4.2,-1$ & .950 & & $3.2,-2$ & .363 \\
& $3.2,-1$ & .908 & & $2.4,-2$ & .285 \\
& $2.4,-1$ & .852 & & $1.8,-2$ & .210 \\
& $1.8,-1$ & .790 & & $1.3,-2$ & .136 \\
& $1.3,-1$ & .716 & & $1.0,-2$ & .086 \\
& & & $7.5-3$ & .046 \\
\hline
\end{tabular}

U.S. GOVERNMENT PRINTING OFFICE : $1966 \quad 0-791-598$ 


\section{Measurement of}

Hydraulic Diffusivity of

Wedge-Shaped Aquifers

Drained by Streams

GEOLOGIGAL SURVEY PROFESAIONAL PAPER 514

PLATES 1-120

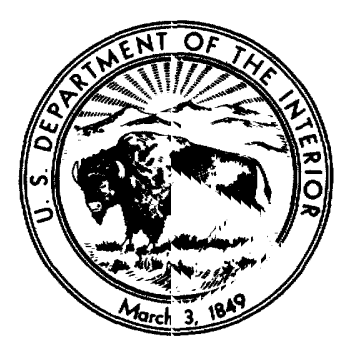


UNITED STATES DEPARTMENT OF THE INTERIOR GEOLOGICAL SURVEY

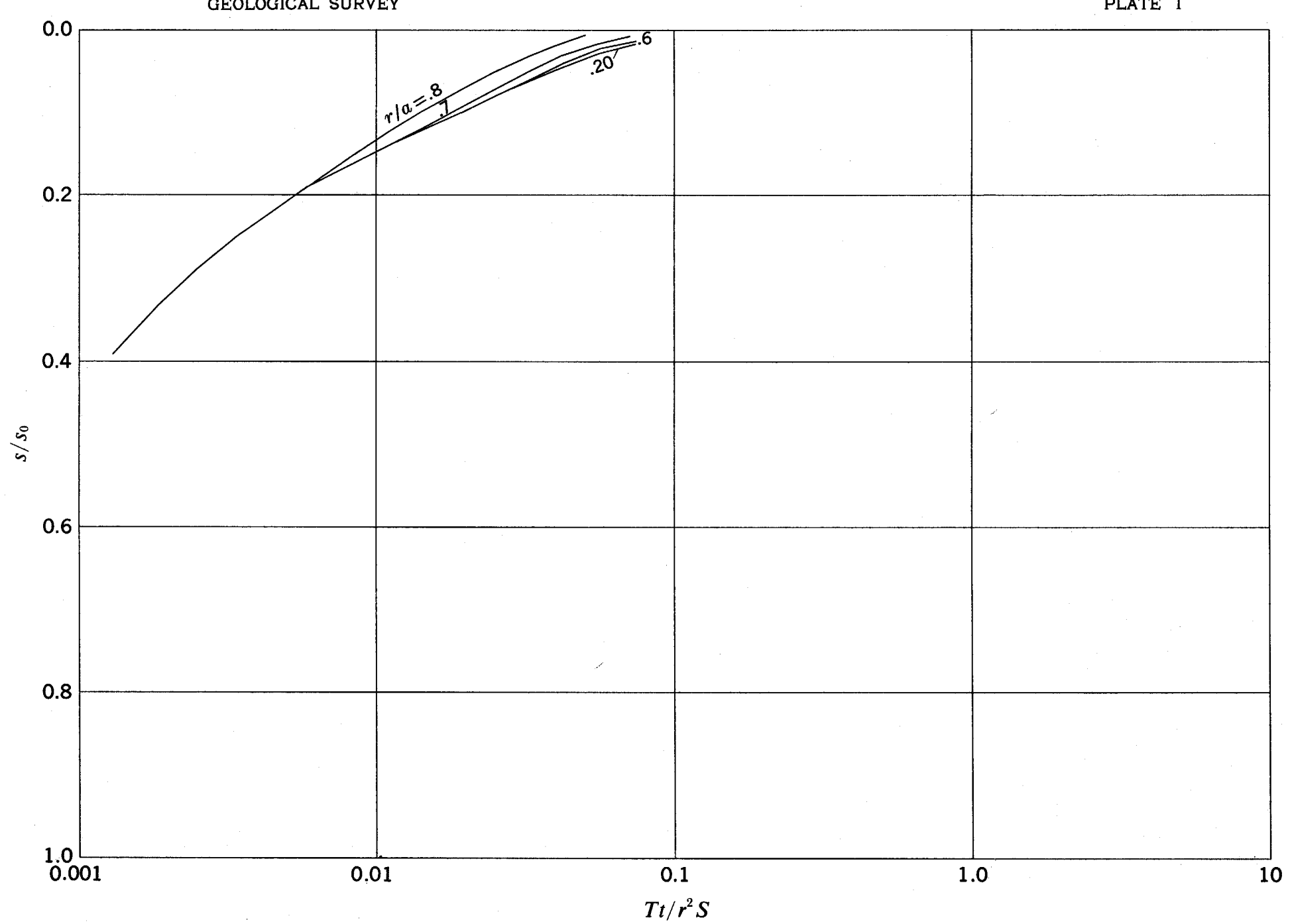

$s / s_{0}$ versus $T t / r^{2} S$ for $\theta_{0}=30^{\circ} ; \theta / \theta_{0}=0.05$ 
UNITED STATES DEPARTMENT OF THE INTERIOR GEOLOGICAL SURVEY

PROFESSIONAL PAPER 514 PLATE 2

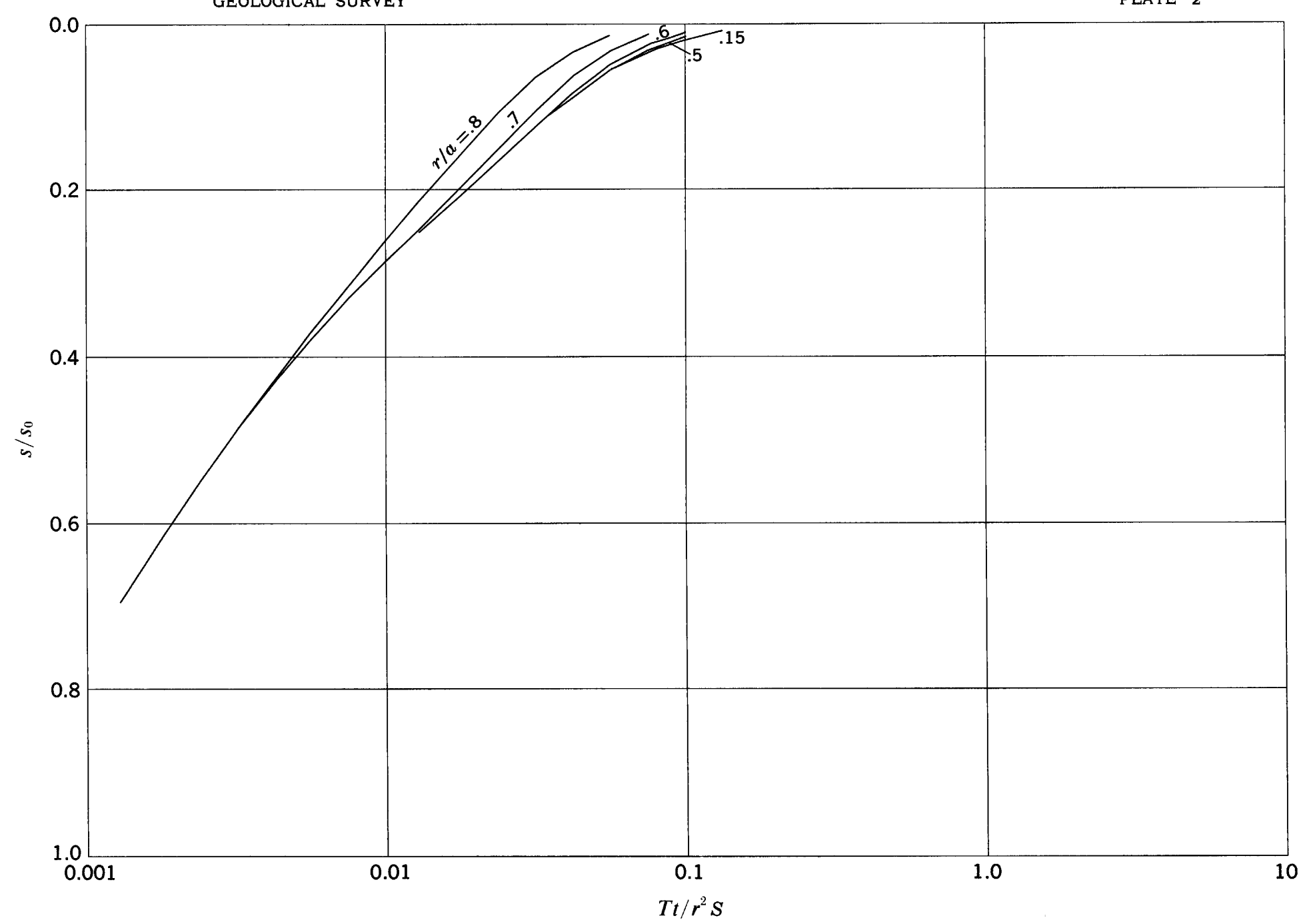

$s / s_{0}$ versus $T t / r^{2} S$ for $\theta_{0}=30^{\circ} ; \theta / \theta_{0}=0.10$ 


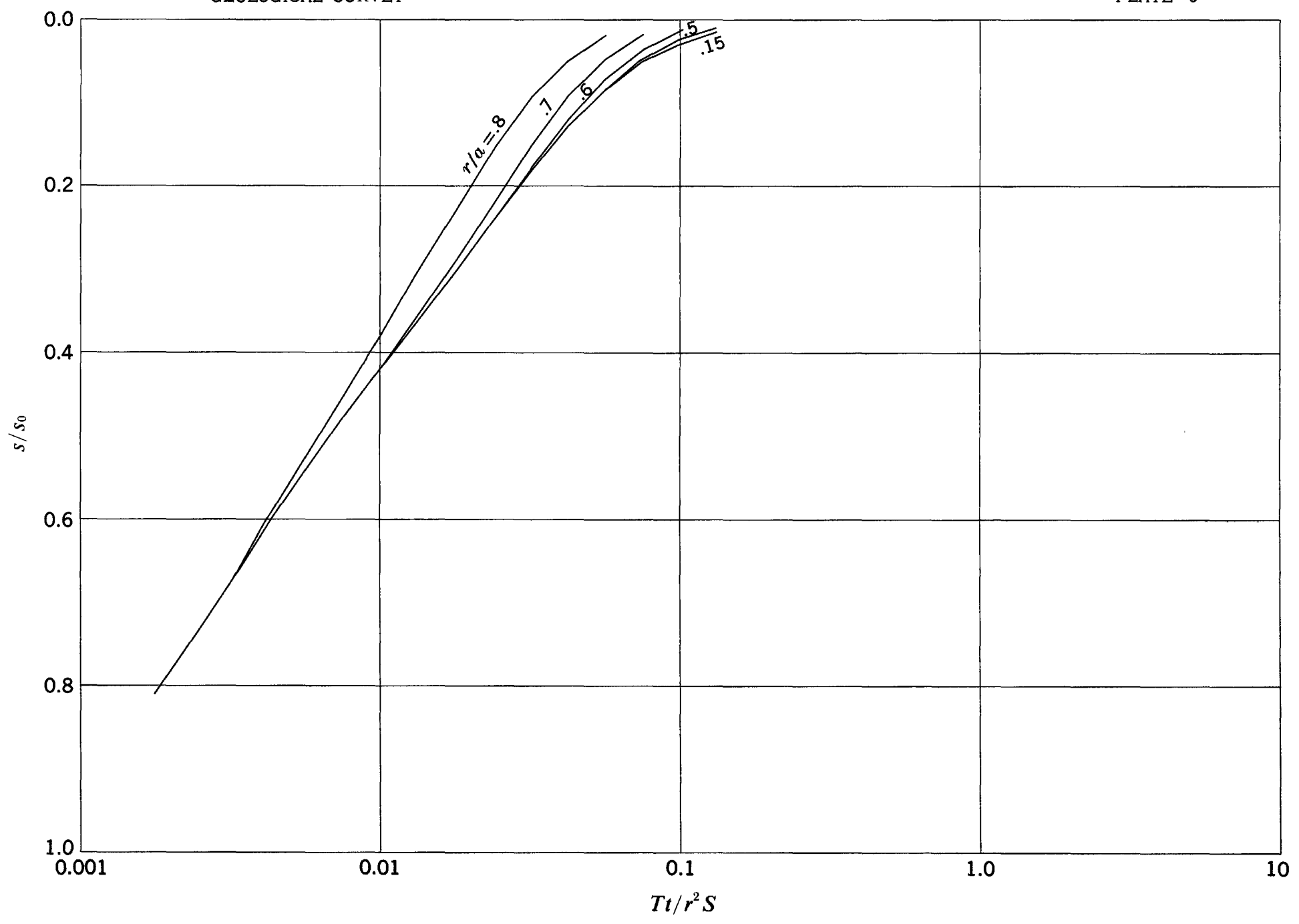

$s / s_{0}$ versus $T t / r^{2} S$ for $\theta_{0}=30^{\circ} ; \theta / \theta_{0}=0.15$ 

GEOLOGICAL SURVEY PLATE 4

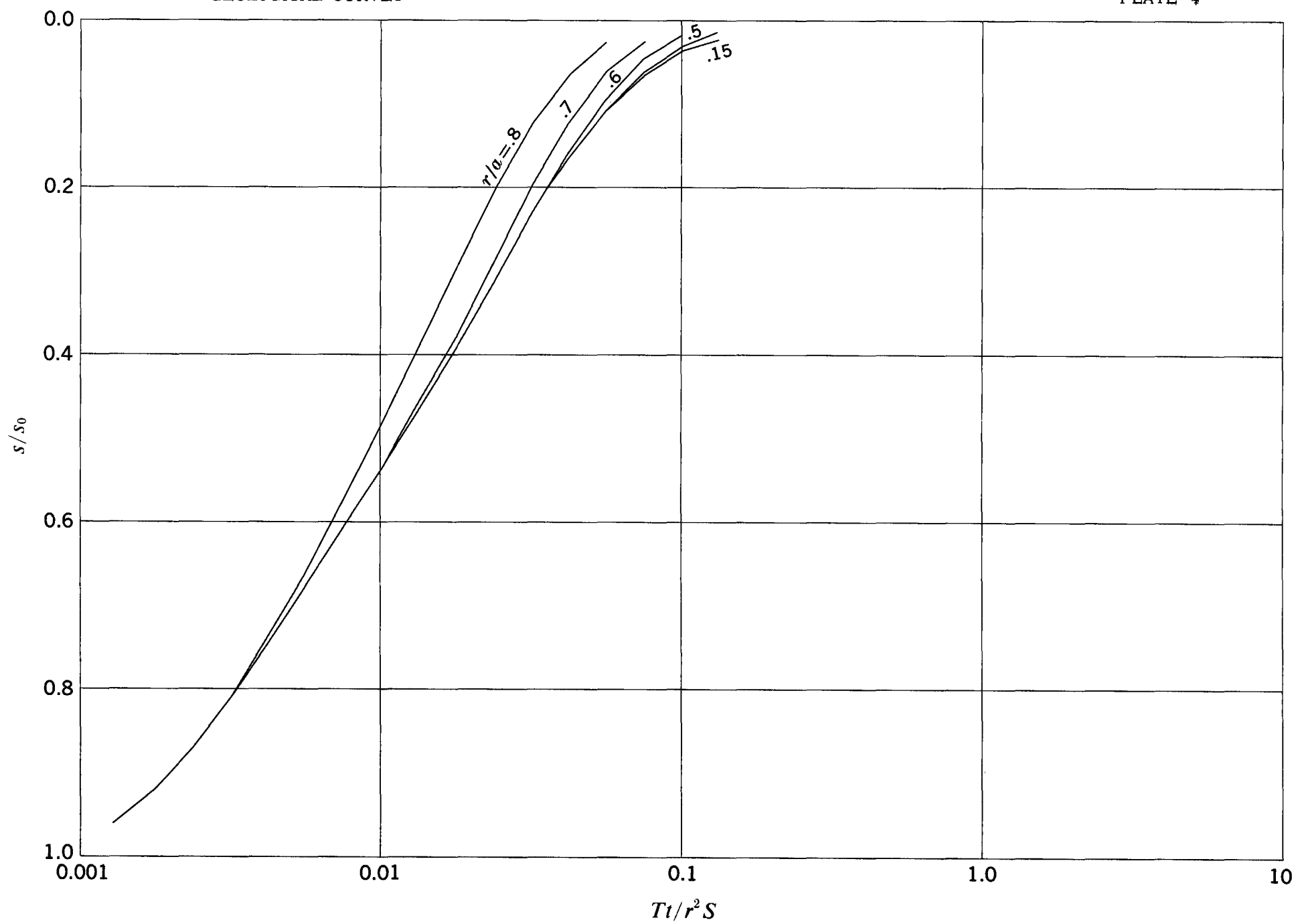

$s / s_{0}$ versus $T t / r^{2} S$ for $\theta_{0}=30^{\circ} ; \theta / \theta_{0}=0.20$ 
UNITED STATES DEPARTMENT OF THE INTERIOR GEOLOGICAL SURVEY
PROFESSIONAL PAPER 514

PLATE 5

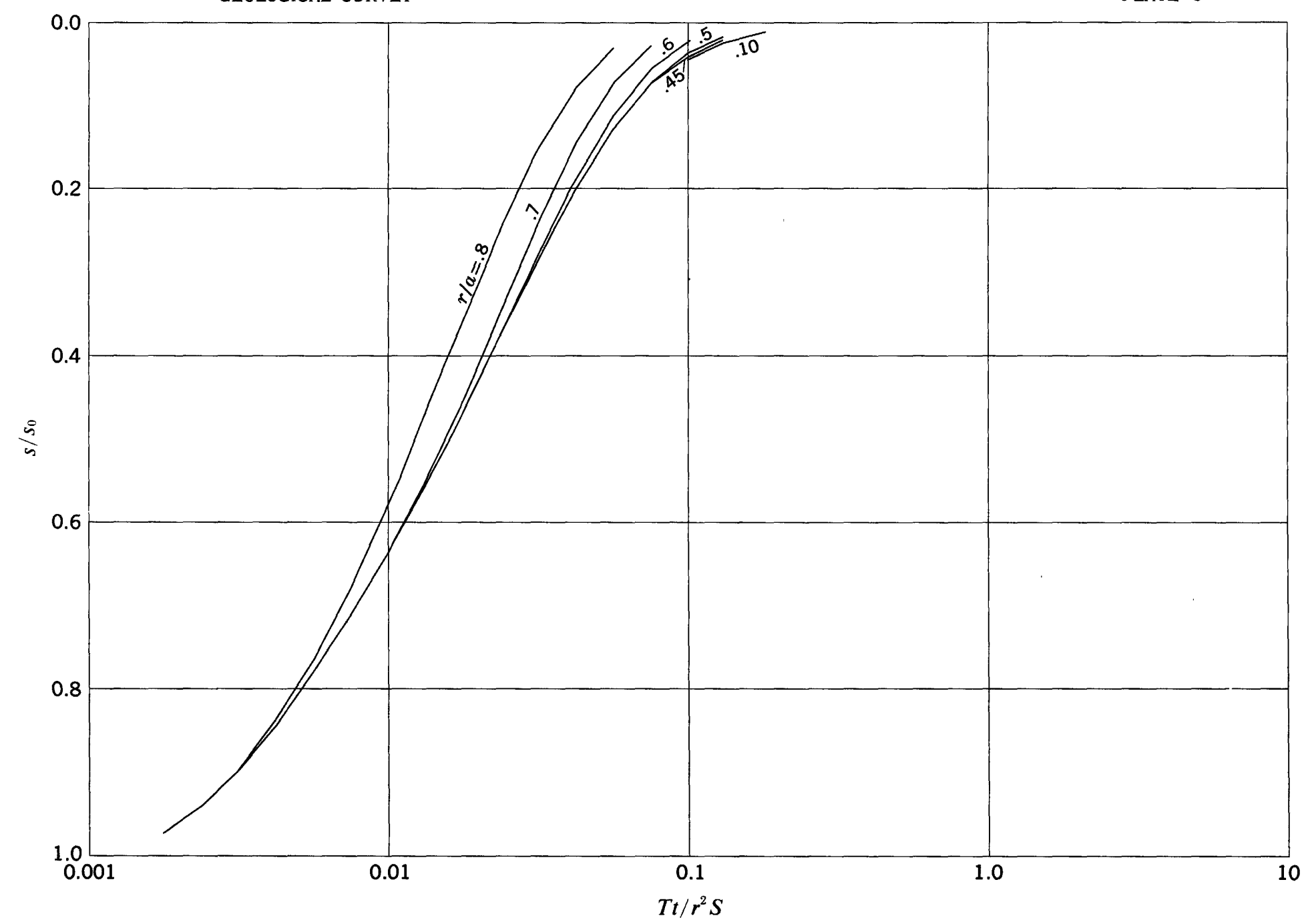

$s / s_{0}$ versus $T t / r^{2} S$ for $\theta_{0}=30^{\circ} ; \theta / \theta_{0}=0.25$ 
UNITED STATES DEPARTMENT OF THE INTERIOR GEOLOGICAL SURVEY
PROFESSIONAL PAPER 514 PLATE 6

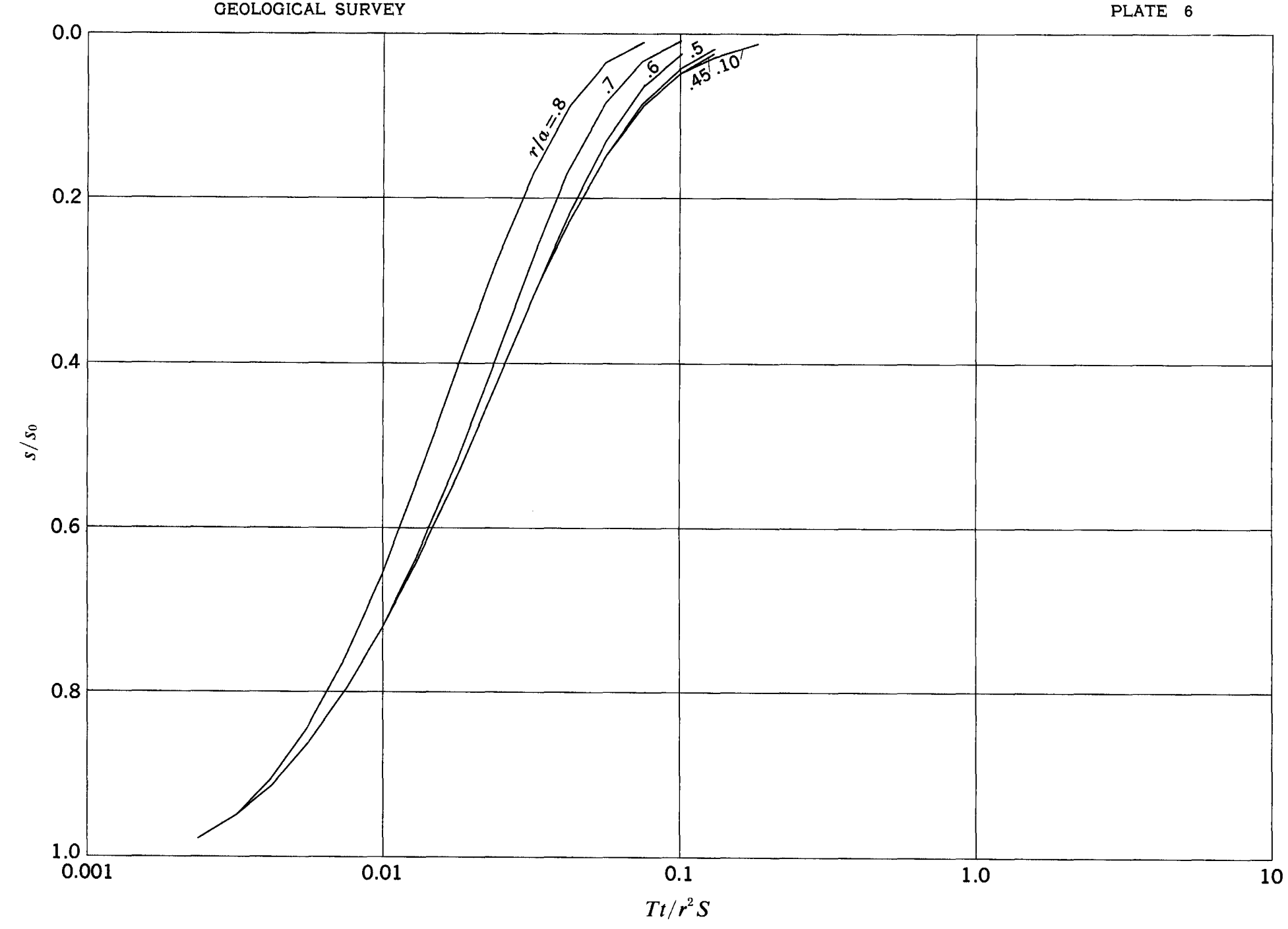

$s / s_{0}$ versus $T t / r^{2} S$ for $\theta_{0}=30^{\circ} ; \theta / \theta_{0}=0.30$ 


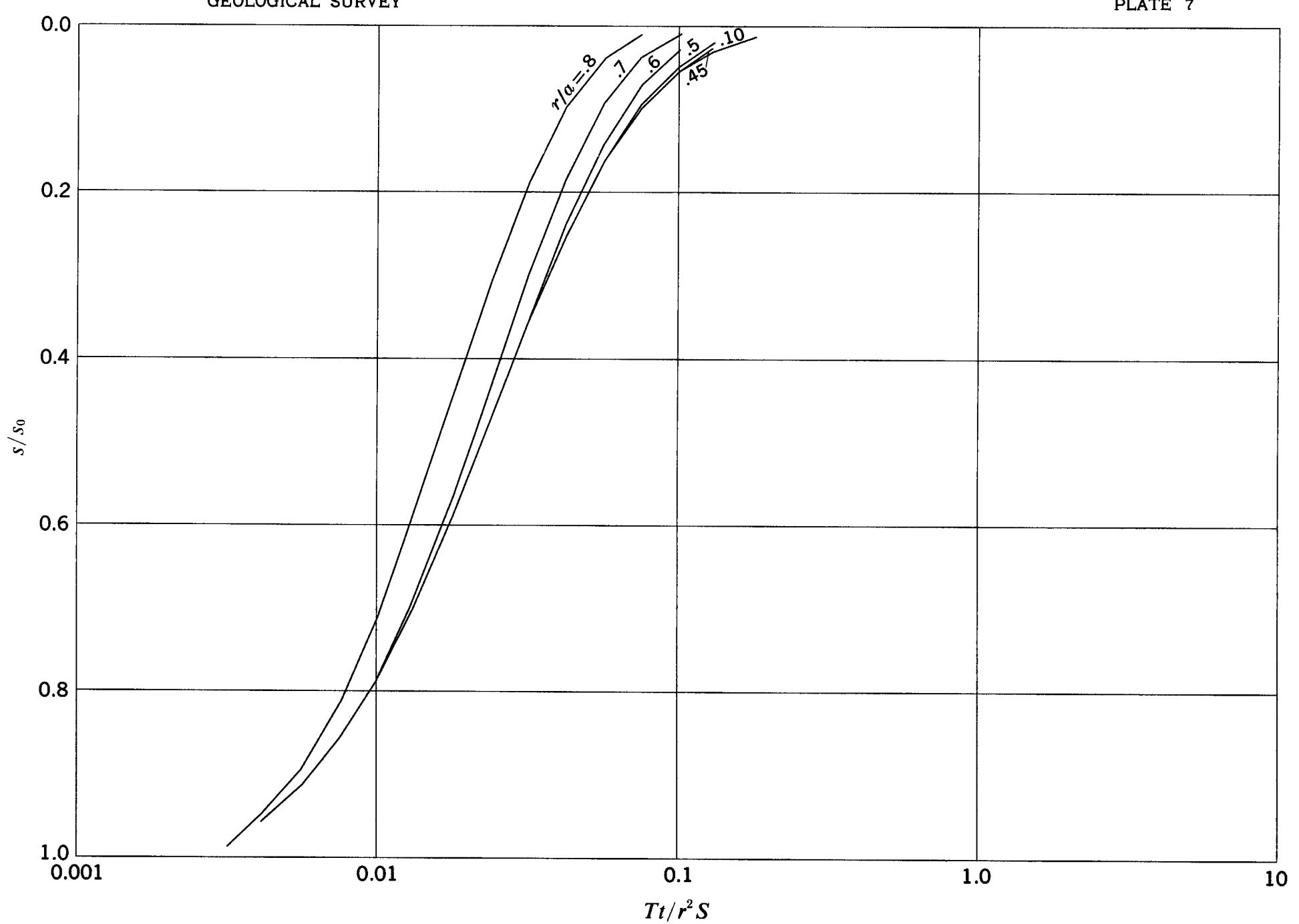

$s / s_{0}$ versus $T t / r^{2} S$ for $\theta_{0}=30^{\circ} ; \theta / \theta_{0}=0.35$ 
UNITED STATES DEPARTMENT OF THE INTERIOR GEOLOGICAL SURVEY
PROFESSIONAL PAPER 514 PLATE 8

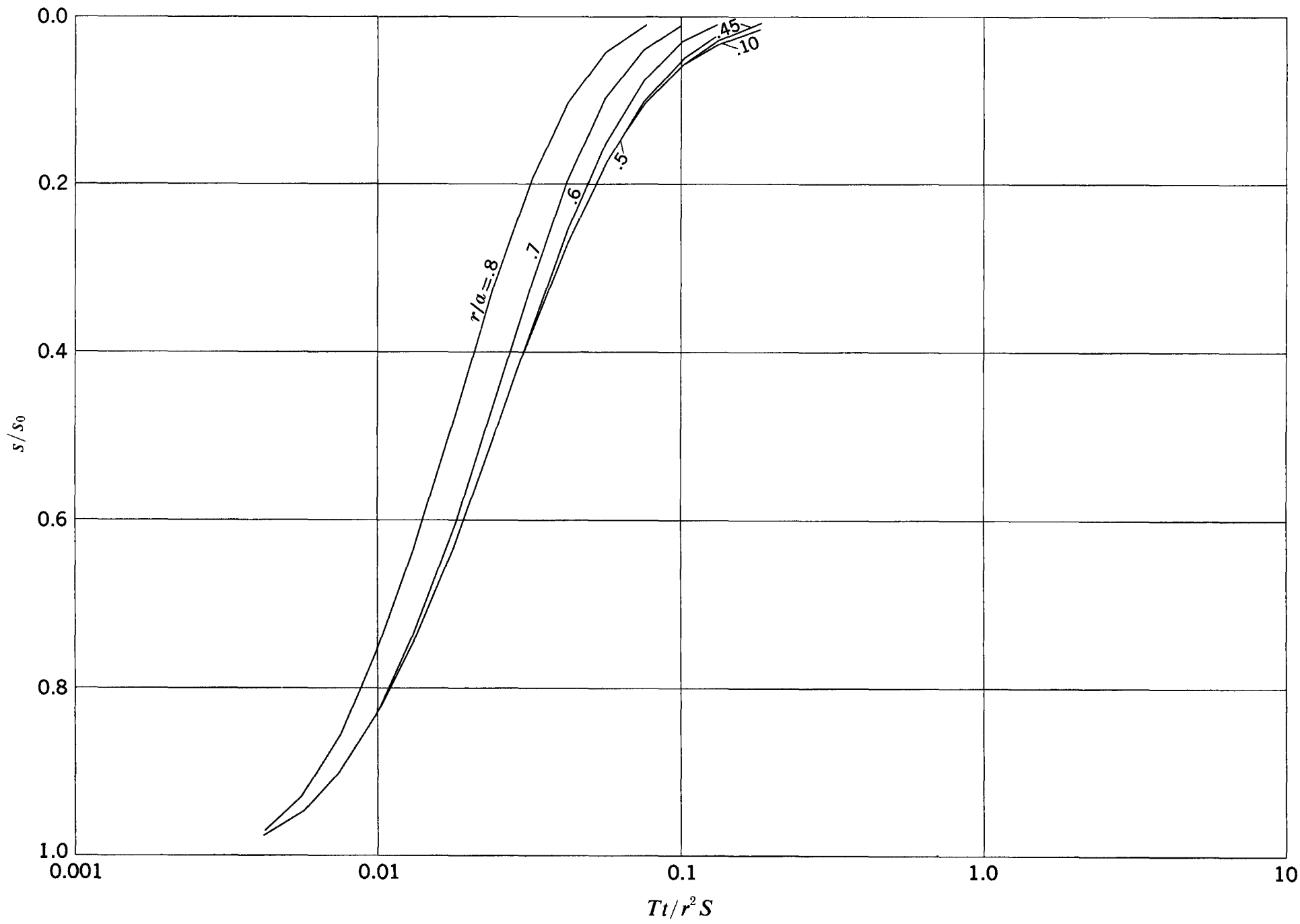

$s / s_{0}$ versus $T t / r^{2} S$ for $\theta_{0}=30^{\circ} ; \theta / \theta_{0}=0.40$ 
UNITED STATES DEPARTMENT OF THE INTERIOR GEOLOGICAL SURVEY

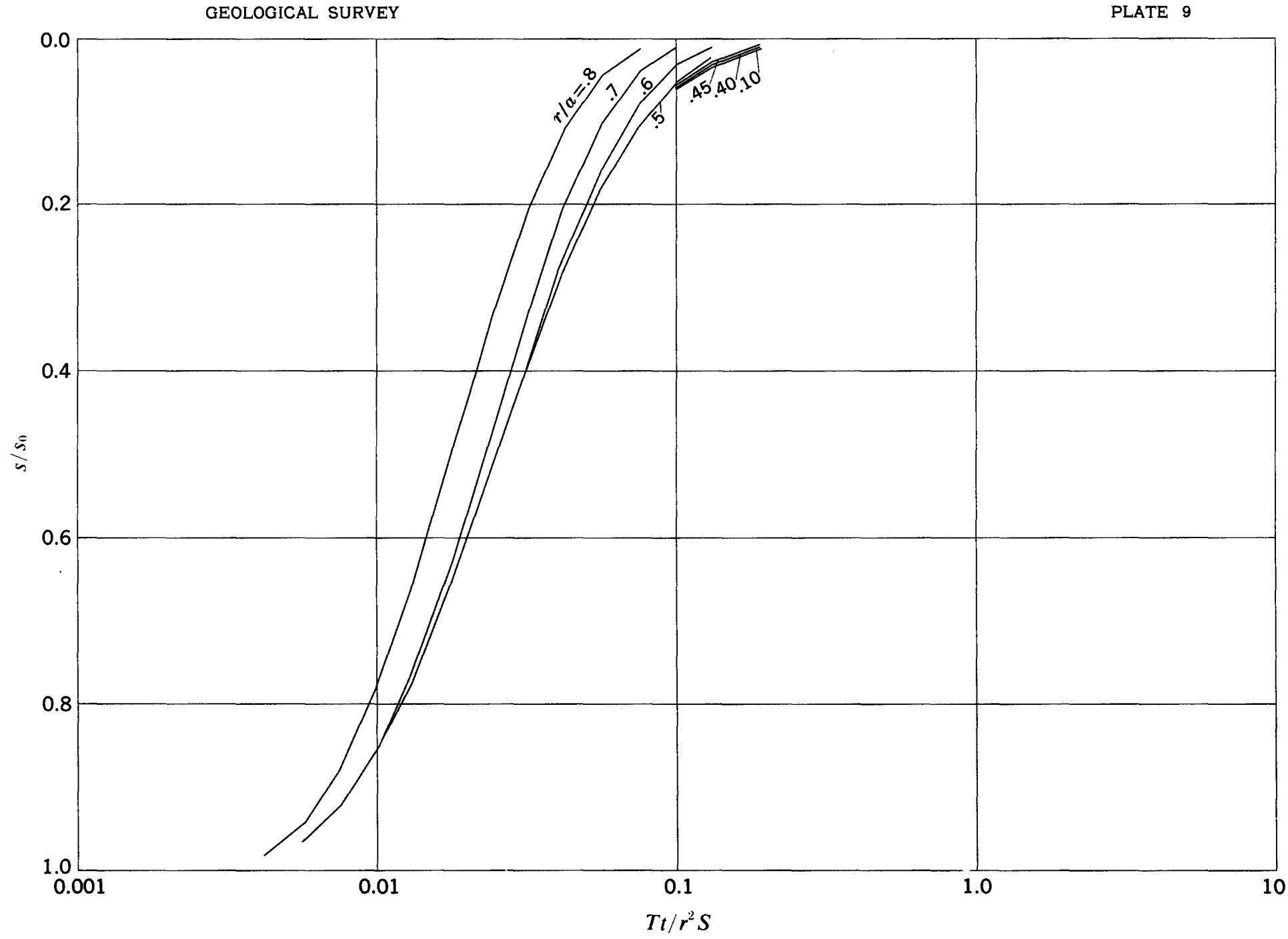

$s / s_{0}$ versus $T t / r^{2} S$ for $\theta_{0}=30^{\circ} ; \theta / \theta_{0}=0.45$ 
UNITED STATES DEPARTMENT OF THE INTERIOR GEOLOGICAL SURVEY

PROFESSIONAL PAPER 514

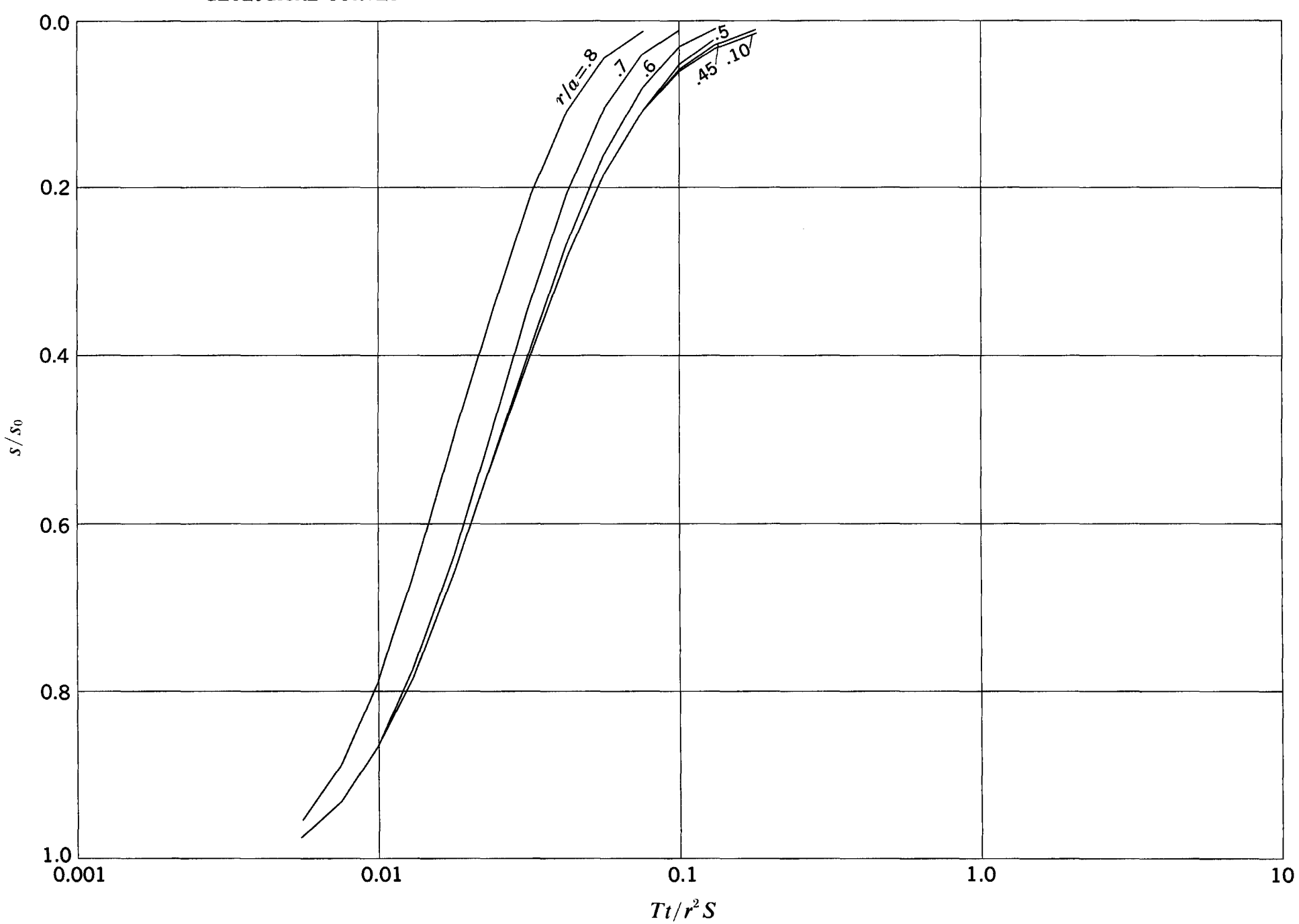

$s / s_{0}$ versus $T t / r^{2} S$ for $\theta_{0}=30^{\circ} ; \theta / \theta_{0}=0.50$ 
UNITED STATES DEPARTMENT OF THE INTERIOR GEOLOGICAL SURVEY

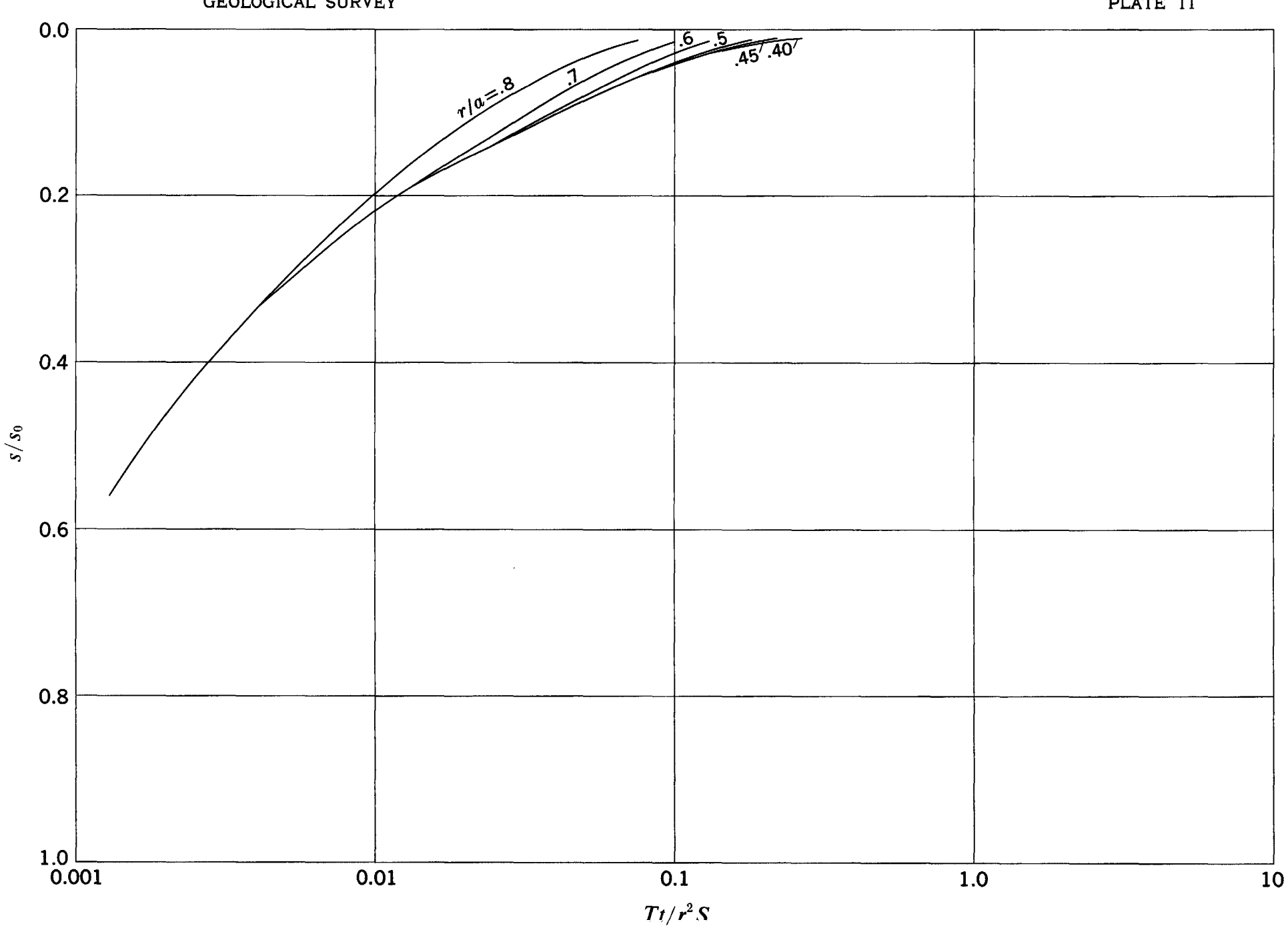

$s / s_{0}$ versus $T t / r^{2} S$ for $\theta_{0}=45^{\circ} ; \theta / \theta_{0}=0.05$ 

GEOLOGICAL SURVEY PLATE 12

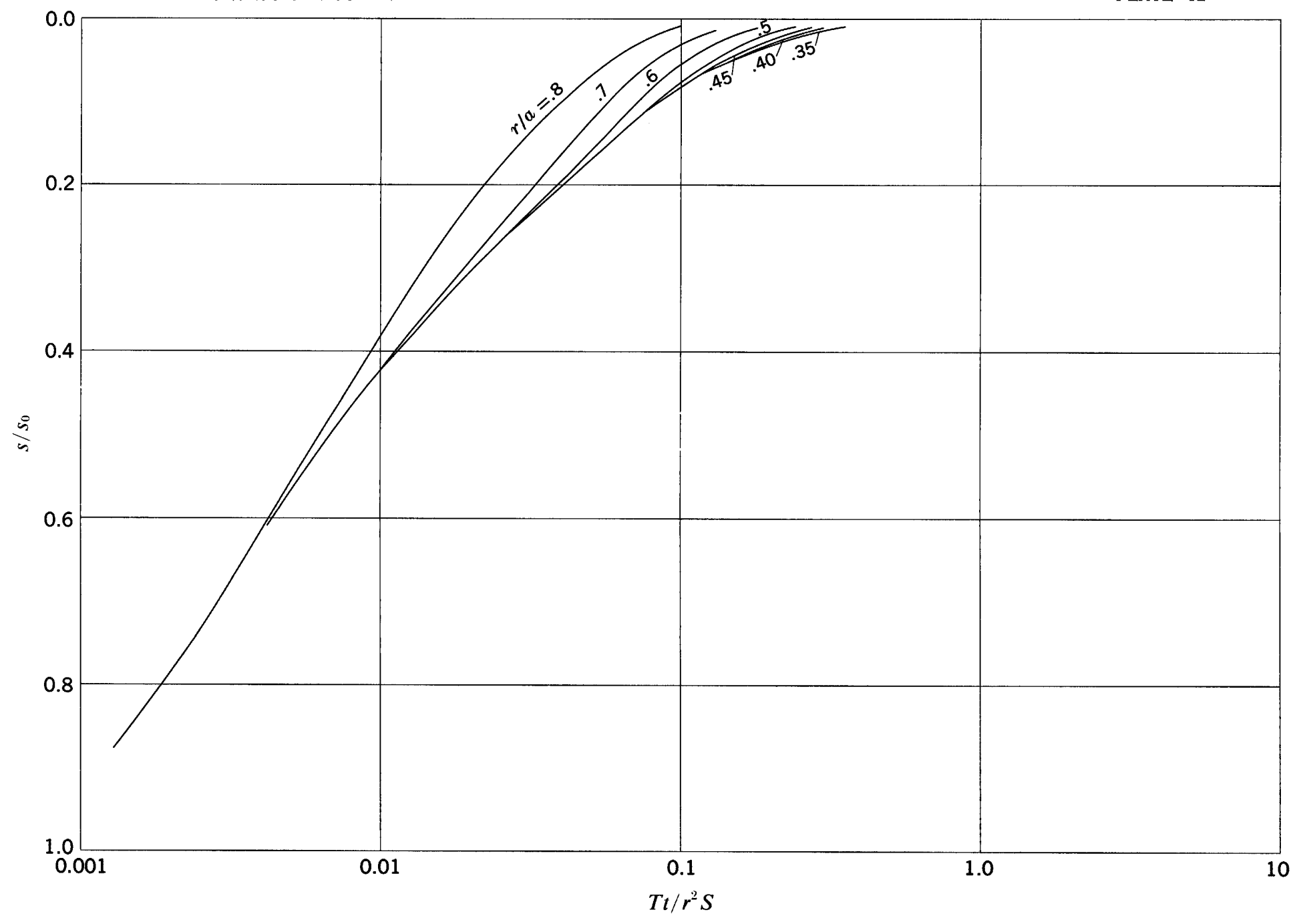

$s / s_{0}$ versus $T t / r^{2} S$ for $\theta_{0}=45^{\circ} ; \theta / \theta_{0}=0.10$ 
UNITED STATES DEPARTMENT OF THE INTERIOR GEOLOGICAL SURVEY

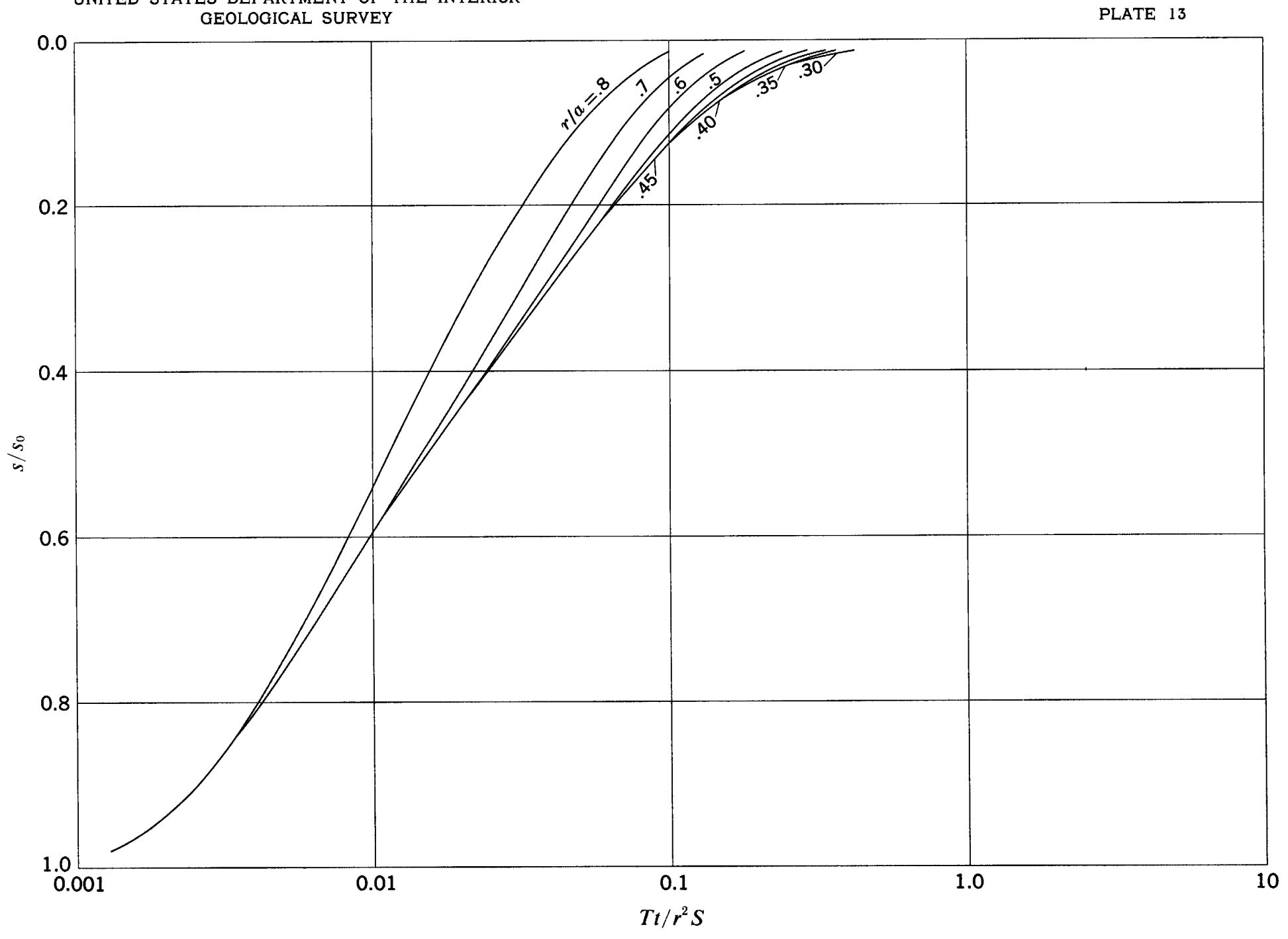

$s / s_{0}$ versus $T t / r^{2} S$ for $\theta_{0}=45^{\circ} ; \theta / \theta_{0}=0.15$
PROFESSIONAL PAPER 514 PLATE 13 


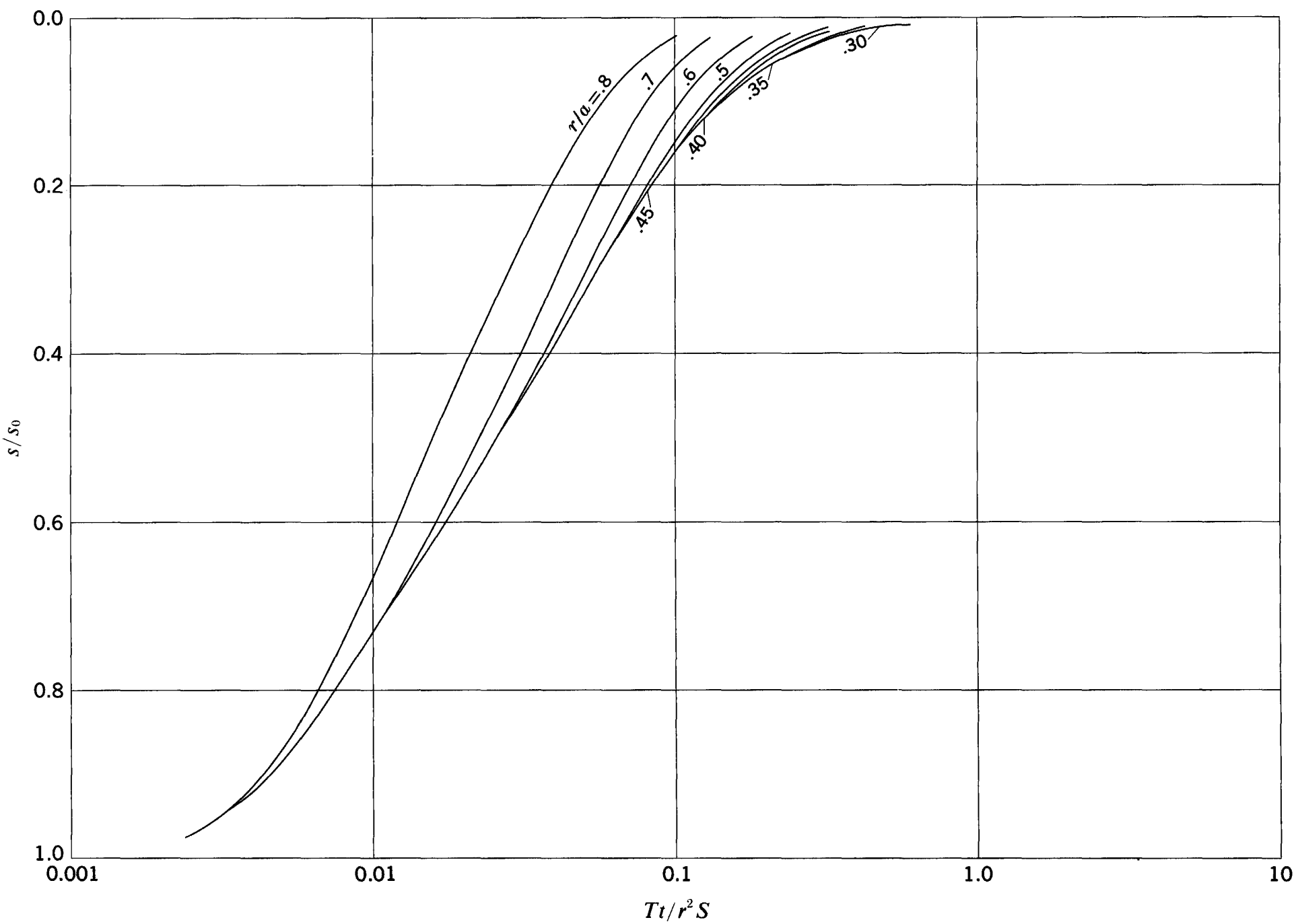

$s / s_{0}$ versus $T t / r^{2} S$ for $\theta_{0}=45^{\circ} ; \theta / \theta_{0}=0.20$ 

GEOLOGICAL SURVEY PLATE 15

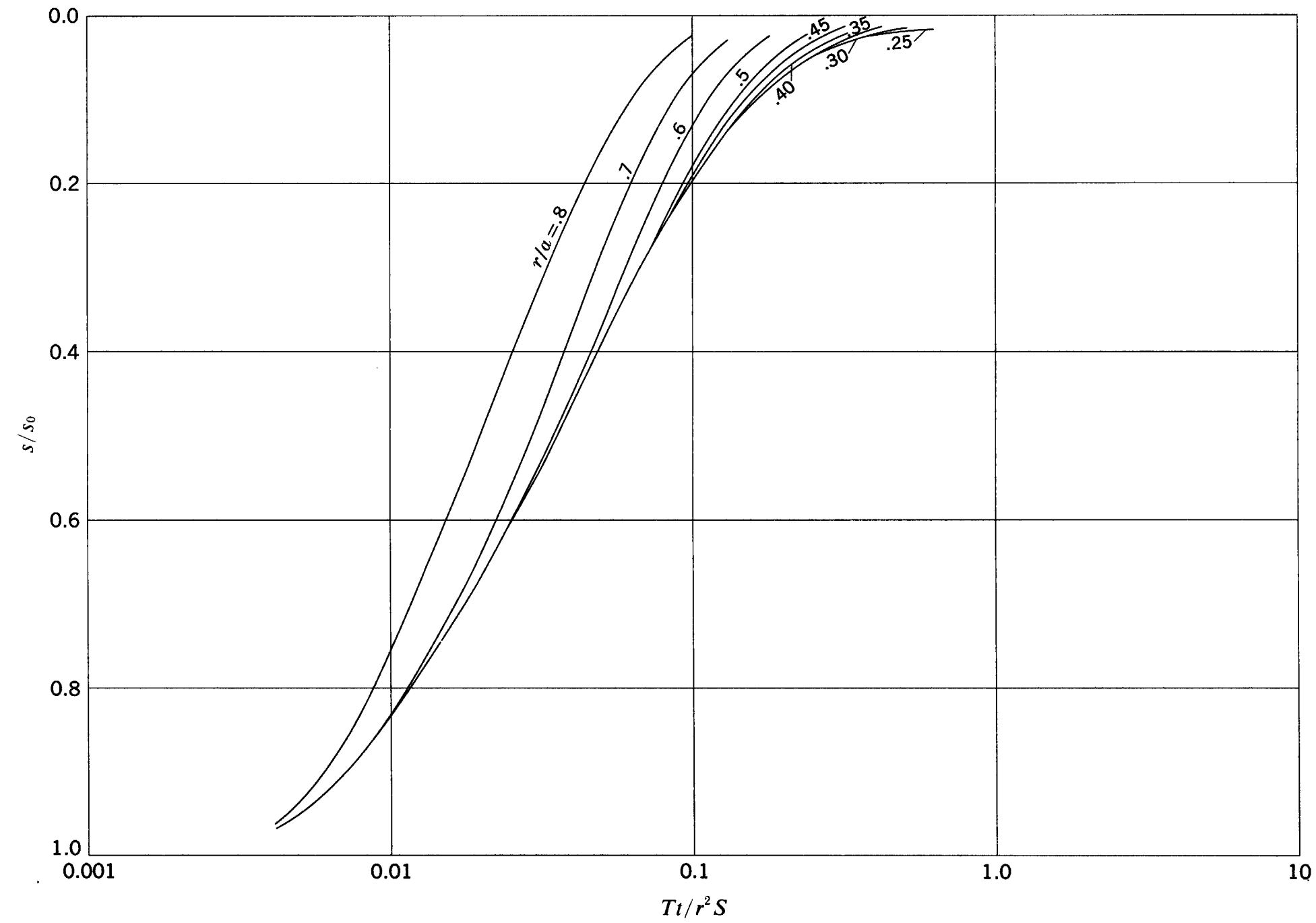

$s / s_{0}$ versus $T t / r^{2} S$ for $\theta_{0}=45^{\circ} ; \theta / \theta_{0}=0.25$ 
UNITED STATES DEPARTMENT OF THE INTERIOR GEOLOGICAL SURVEY

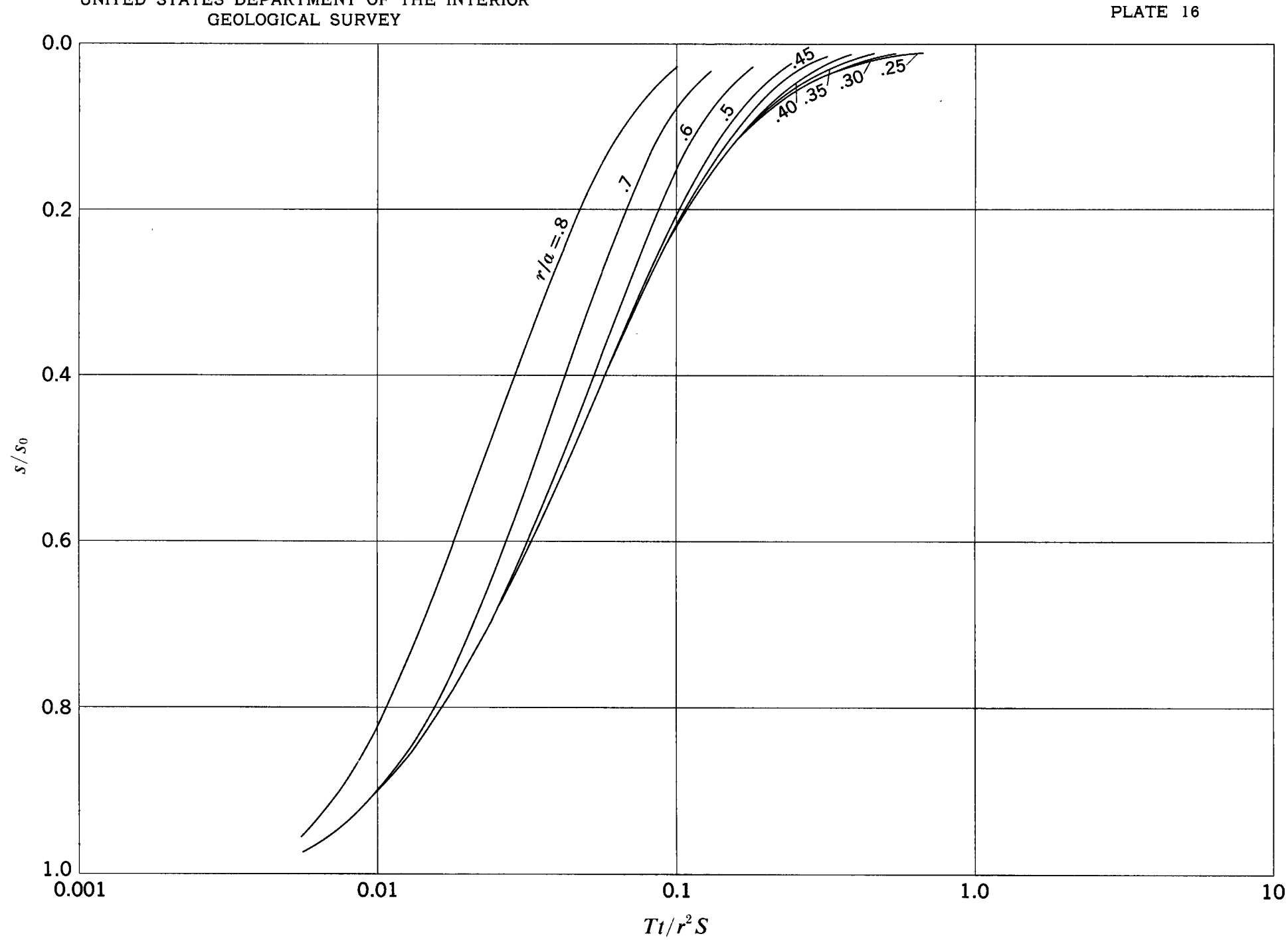

$s / s_{0}$ versus $T t / r^{2} S$ for $\theta_{0}=45^{\circ} ; \theta / \theta_{0}=0.30$ 
UNITED STATES DEPARTMENT OF THE INTERIOR GEOLOGICAL SURVEY

PROFESSIONAL PAPER 514

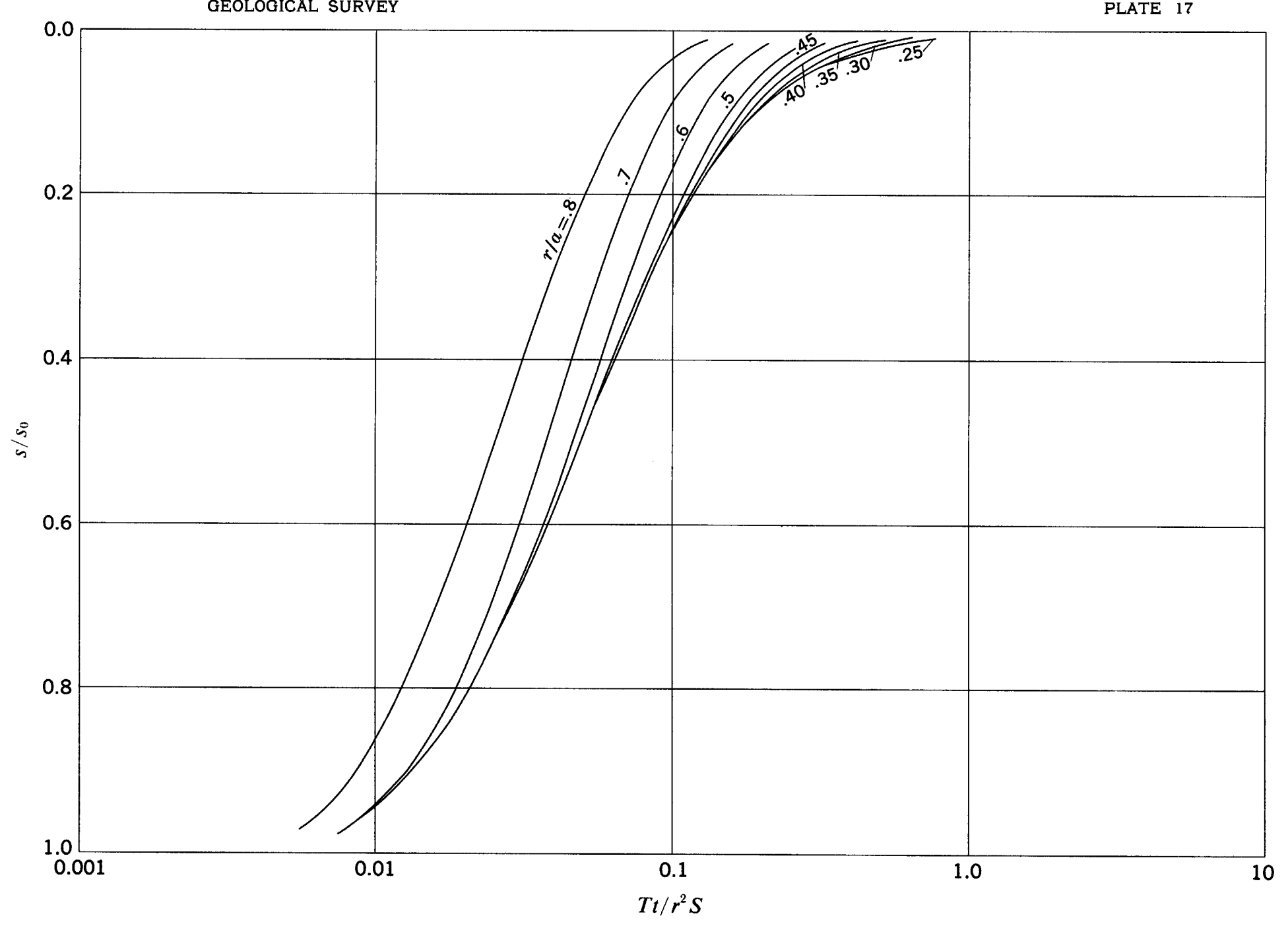

$s / s_{0}$ versus $T t / r^{2} S$ for $\theta_{0}=45^{\circ} ; \theta / \theta_{0}=0.35$ 


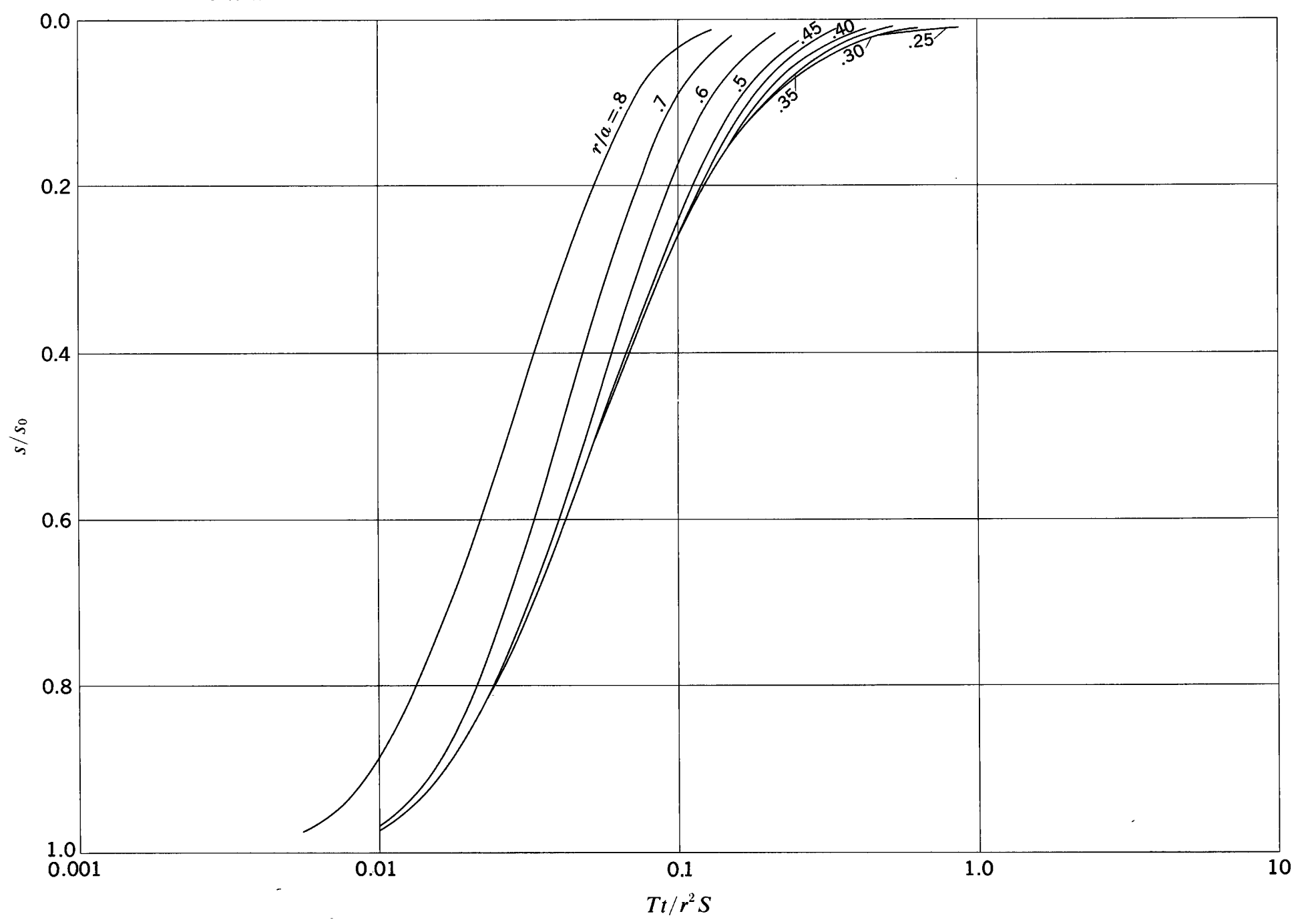

$s / s_{0}$ versus $T t / r^{2} S$ for $\theta_{0}=45^{\circ} ; \theta / \theta_{0}=0.40$ 
UNITED STATES DEPARTMENT OF THE INTERIOR GEOLOGICAL SURVEY

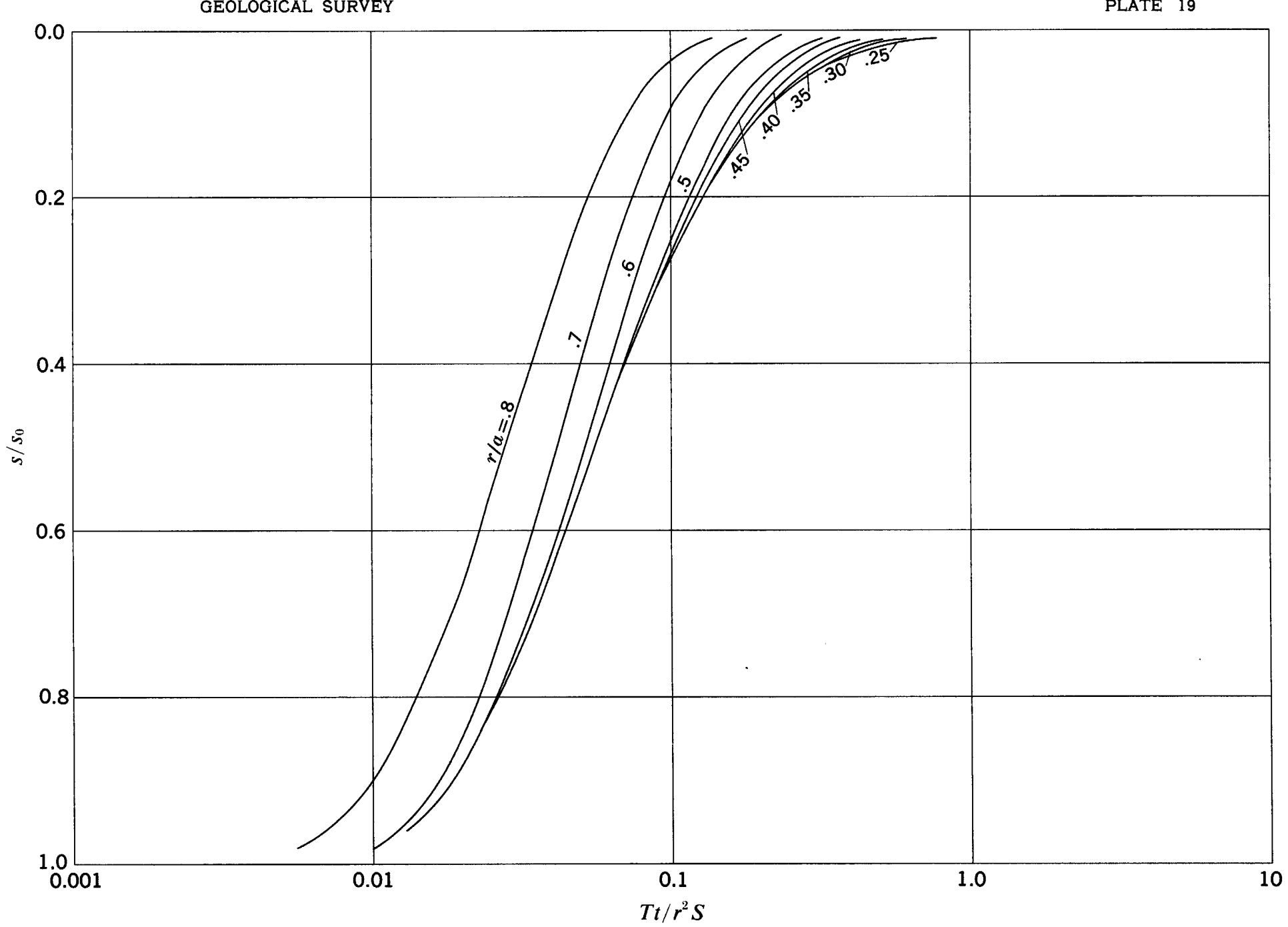

$s / s_{0}$ versus $T t / r^{2} S$ for $\theta_{0}=45^{\circ} ; \theta / \theta_{0}=0.45$ 


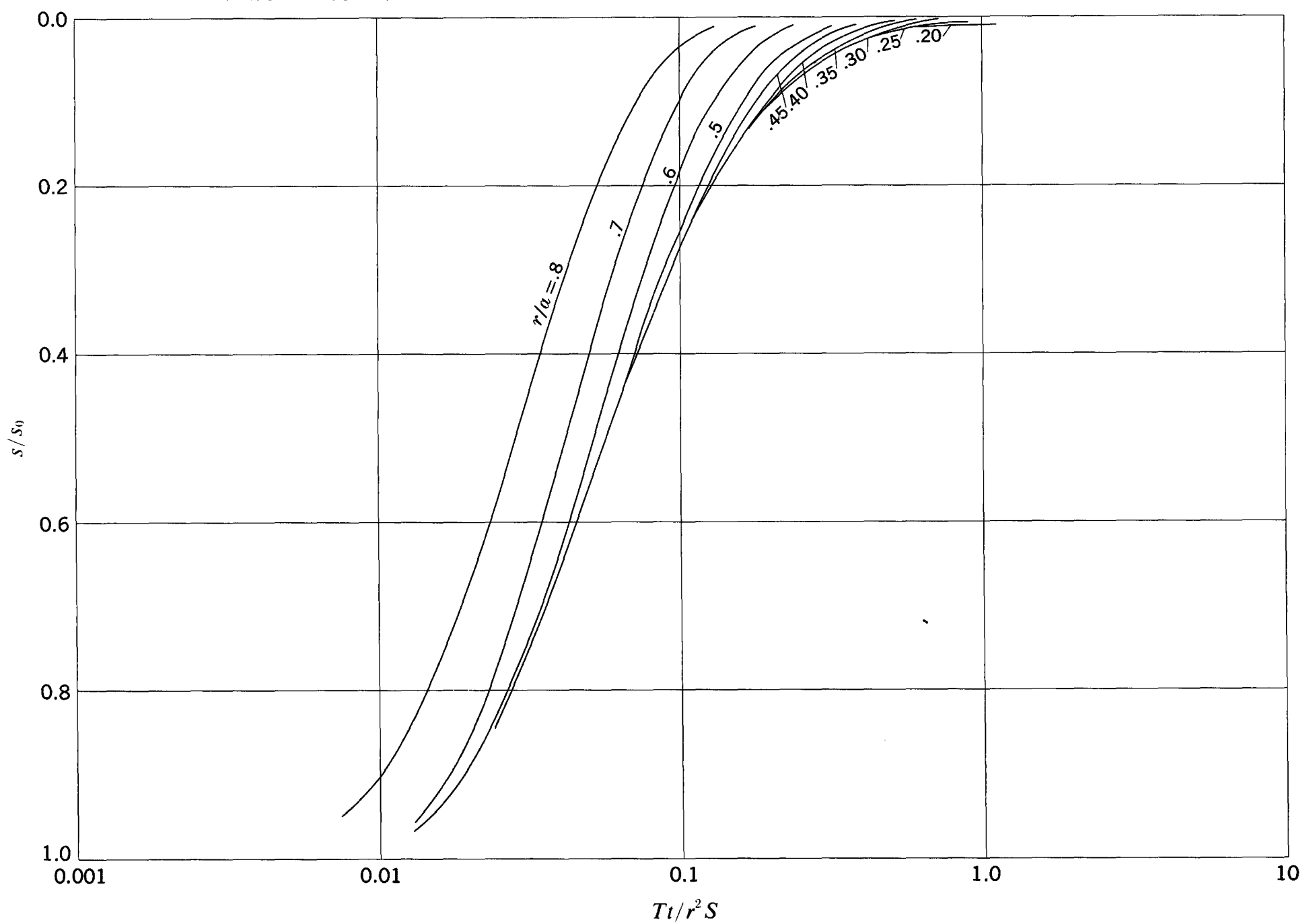

$s / s_{0}$ versus $T t / r^{2} S$ for $\theta_{0}=45^{\circ} ; \theta / \theta_{0}=0.50$ 


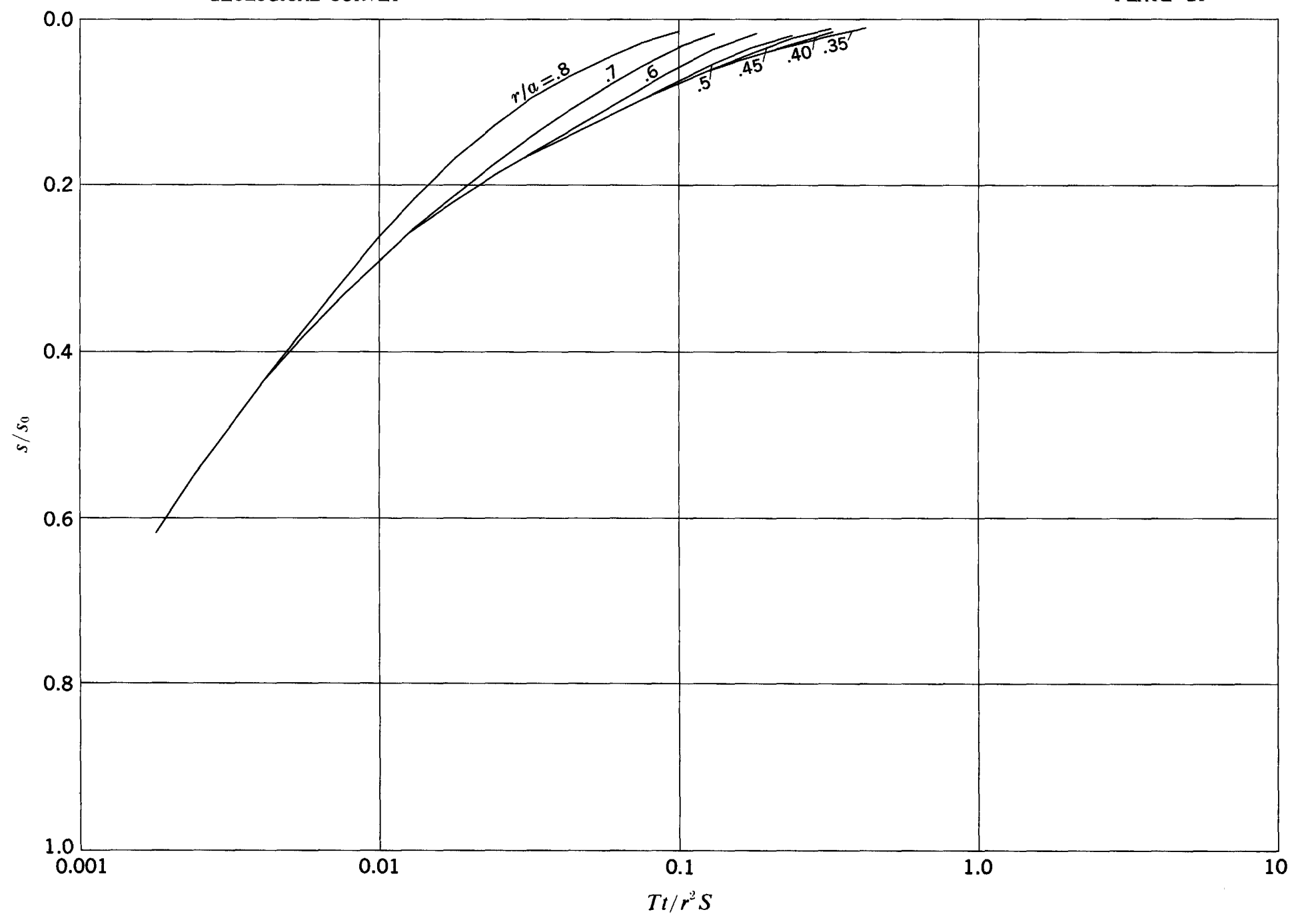

$s / s_{0}$ versus $T t / r^{2} S$ for $\theta_{0}=60^{\circ} ; \theta / \theta_{0}=0.05$ 
UNITED STATES DEPARTMENT OF THE INTERIOR GEOLOGICAL SURVEY

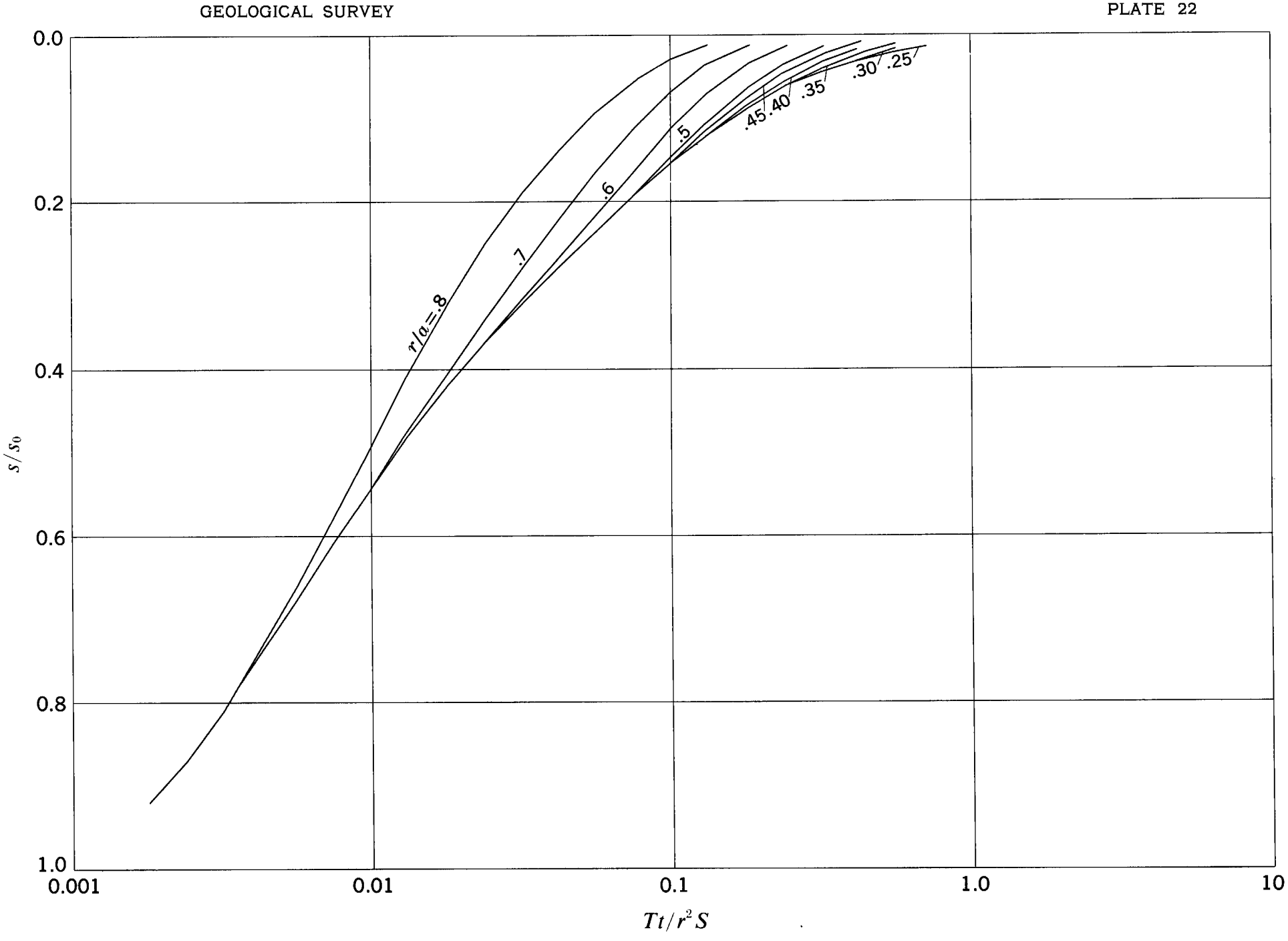

$s / s_{0}$ versus $T t / r^{2} S$ for $\theta_{0}=60^{\circ} ; \theta / \theta_{0}=0.10$
PROFESSIONAL PAPER 514 PLATE 22 
UNITED STATES DEPARTMENT OF THE INTERIOR GEOLOGICAL SURVEY

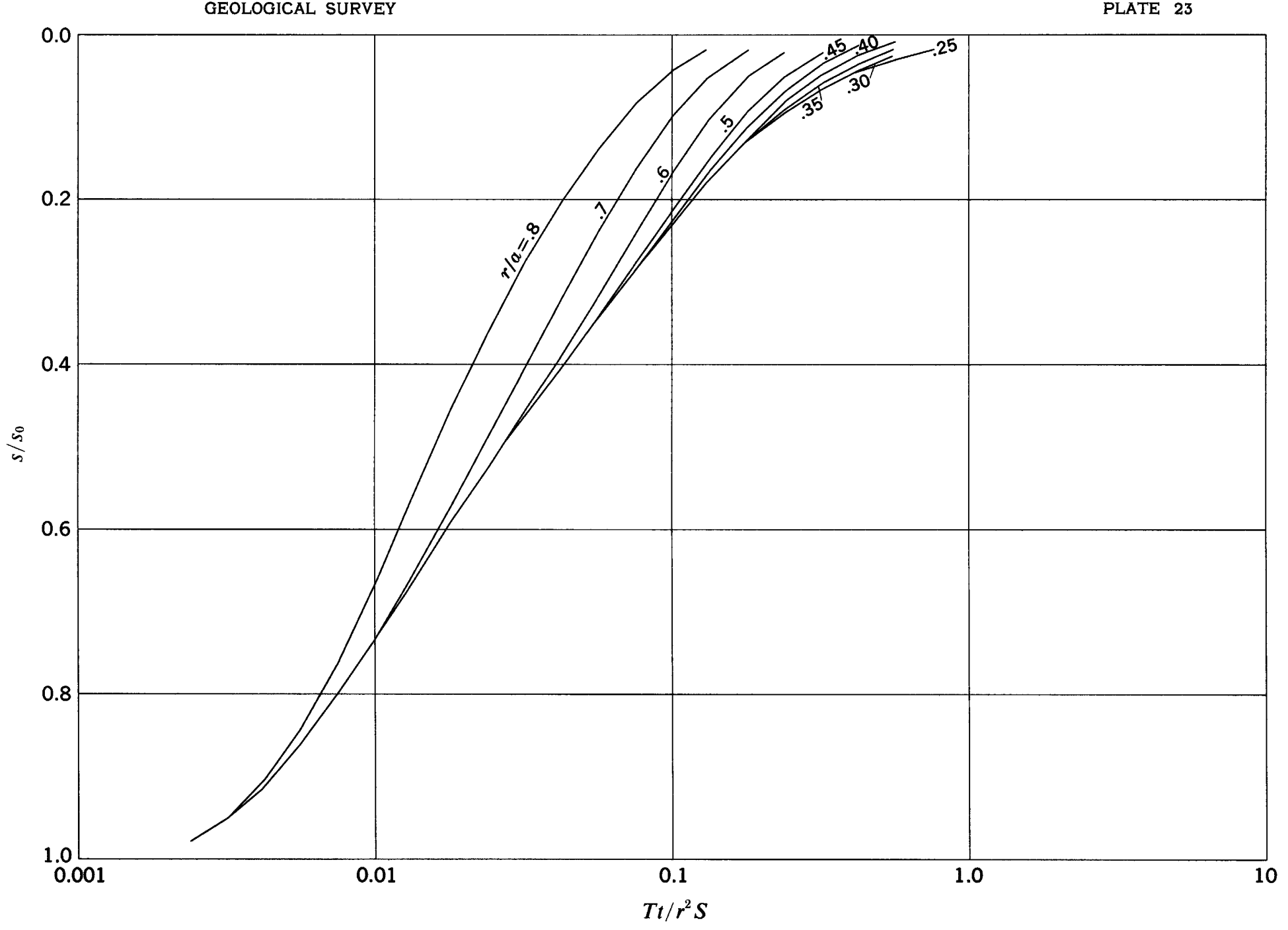

$s / s_{0}$ versus $T t / r^{2} S$ for $\theta_{0}=60^{\circ} ; \theta / \theta_{0}=0.15$ 

GEOLOGICAL SURVEY PLATE 24

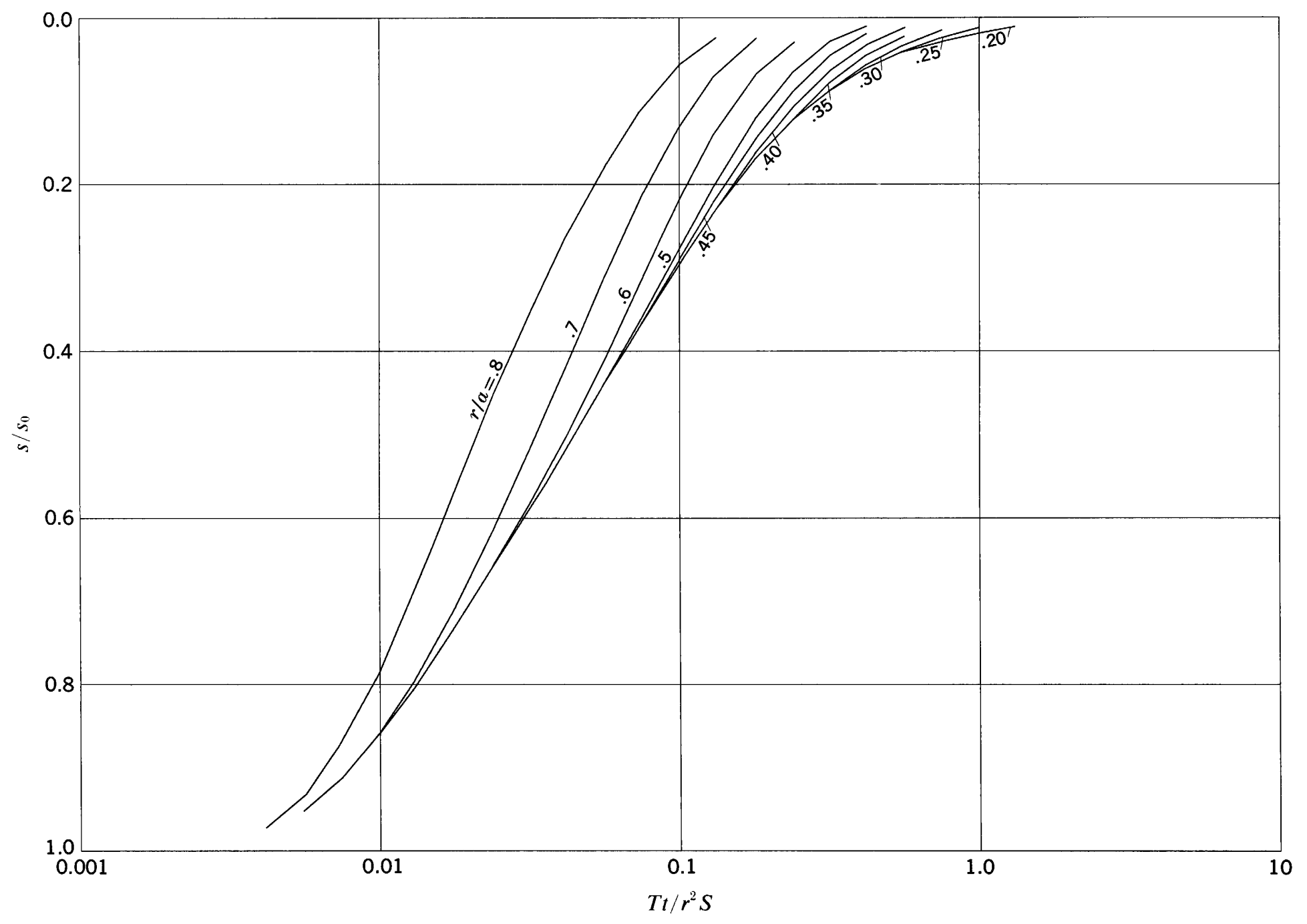

$s / s_{0}$ versus $T t / r^{2} S$ for $\theta_{0}=60^{\circ} ; \theta / \theta_{0}=0.20$ 
UNITED STATES DEPARTMENT OF THE INTERIOR GEOLOGICAL SURVEY
PROFESSIONAL PAPER 514 PLATE 25

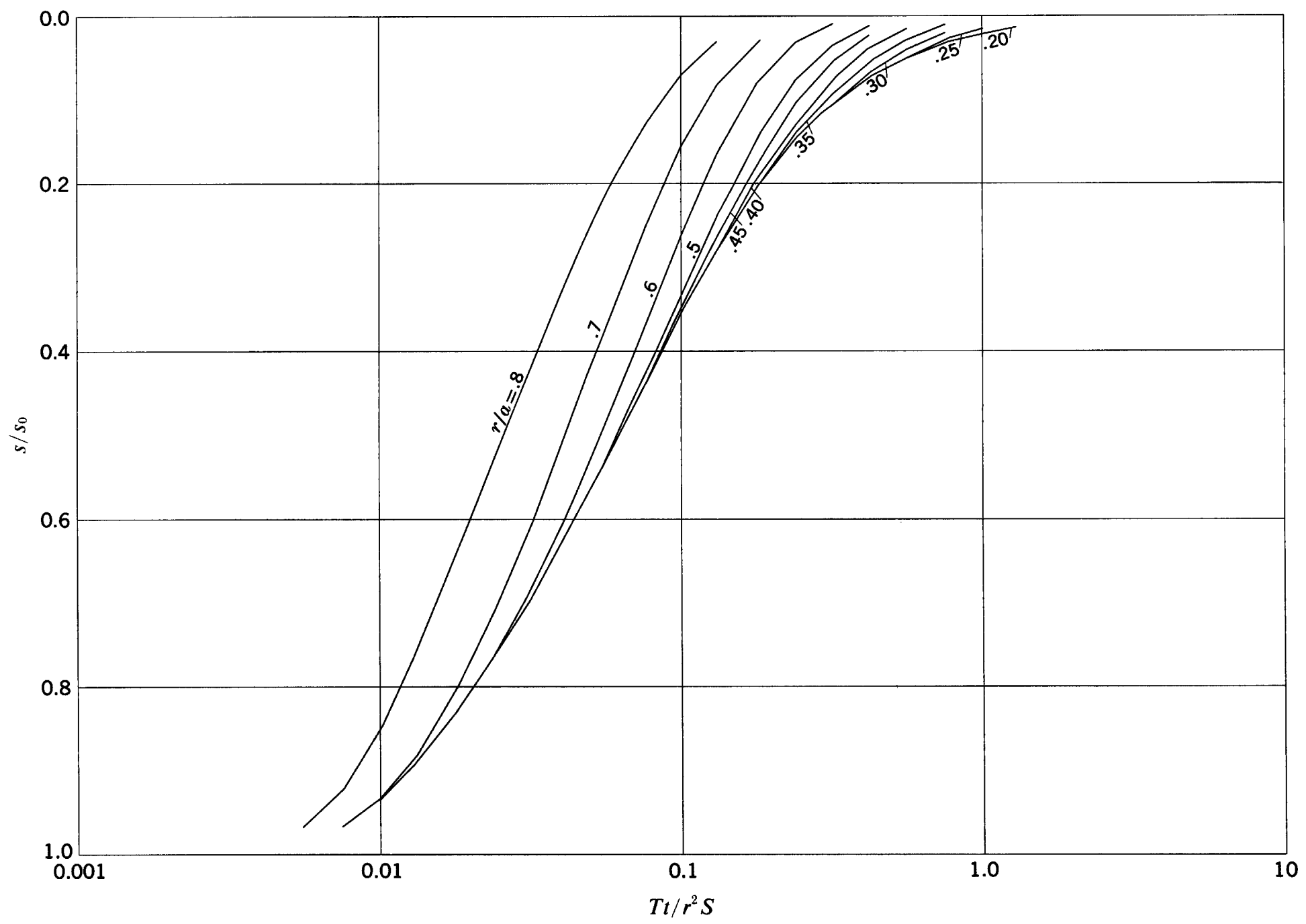

$s / s_{0}$ versus $T t / r^{2} S$ for $\theta_{0}=60^{\circ} ; \theta / \theta_{0}=0.25$ 

GEOLOGICAL SURVEY PLATE 26

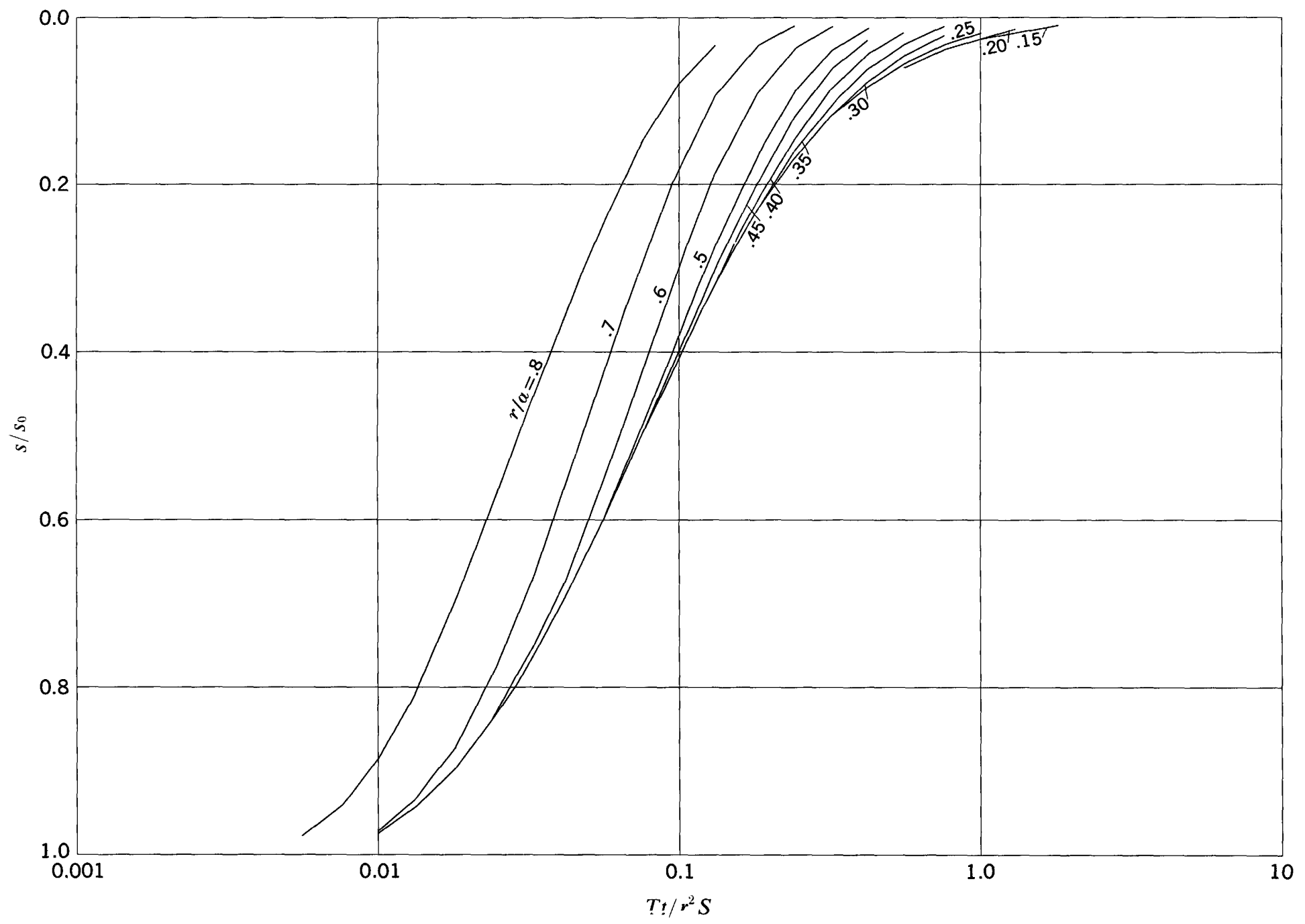

$s / s_{0}$ versus $T t / r^{2} S$ for $\theta_{0}=60^{\circ} ; \theta / \theta_{0}=0.30$ 

GEOLOGICAL SURVEY 


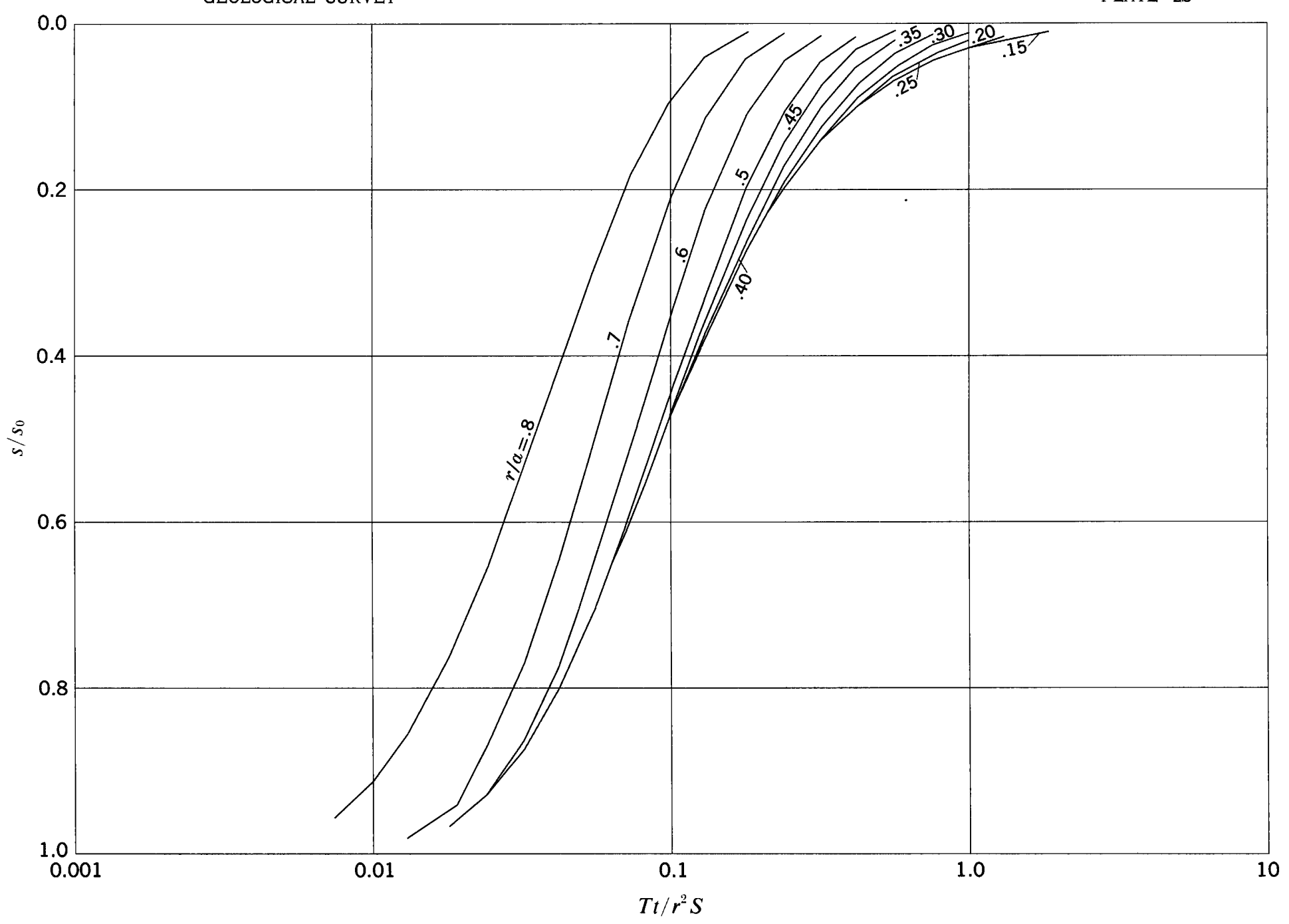

$s / s_{0}$ versus $T t / r^{2} S$ for $\theta_{0}=60^{\circ} ; \theta / \dot{\theta}_{0}=0.40$ 
UNITED STATES DEPARTMENT OF THE INTERIOR GEOLOGICAL SURVEY

PROFESSIONAL PAPER 514 PLATE 29

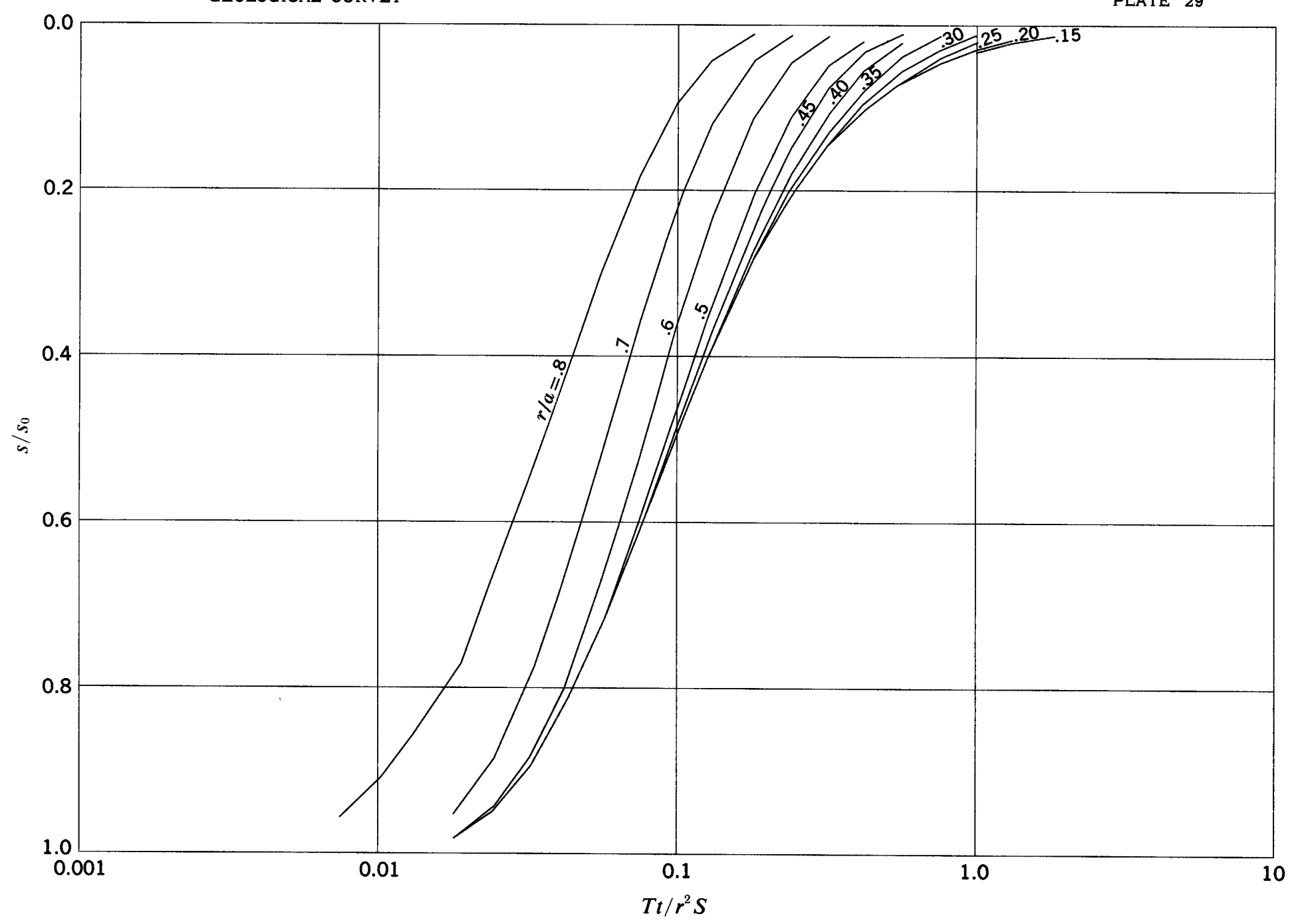

$s / s_{0}$ versus $T t / r^{2} S$ for $\theta_{0}=60^{\circ} ; \theta / \theta_{0}=0.45$ 

GEOLOGICAL SURVEY PLATE 30

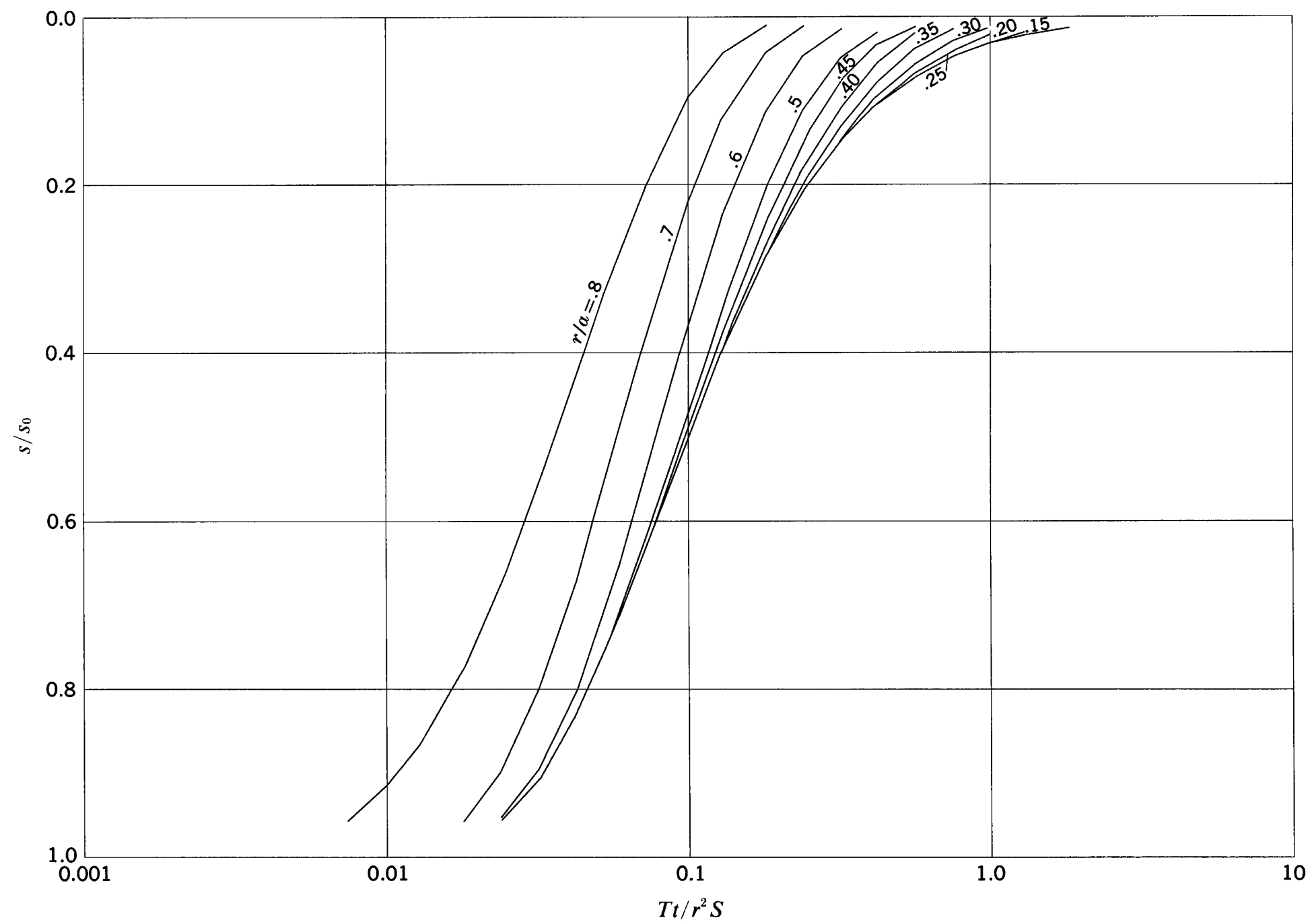

$s / s_{0}$ versus $T t / r^{2} S$ for $\theta_{0}=60^{\circ} ; \theta / \theta_{0}=0.50$ 
UNITED STATES DEPARTMENT OF THE INTERIOR GEOLOGICAL SURVEY

PROFESSIONAL PAPER 514 PLATE 31

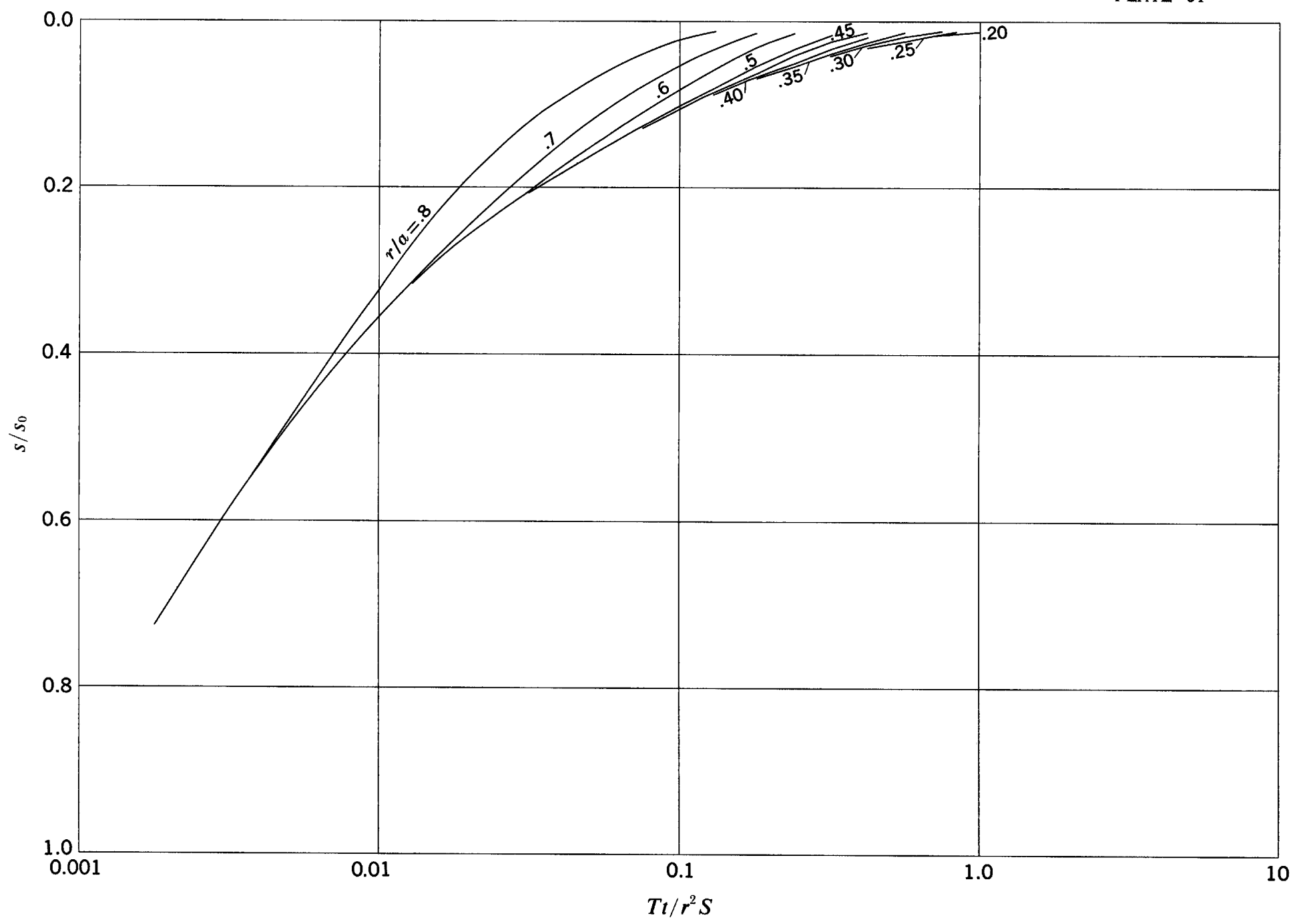

$s / s_{0}$ versus $T t / r^{2} S$ for $\theta_{0}=75^{\circ} ; \theta / \theta_{0}=0.05$ 

GEOLOGICAL SURVEY PLATE 32

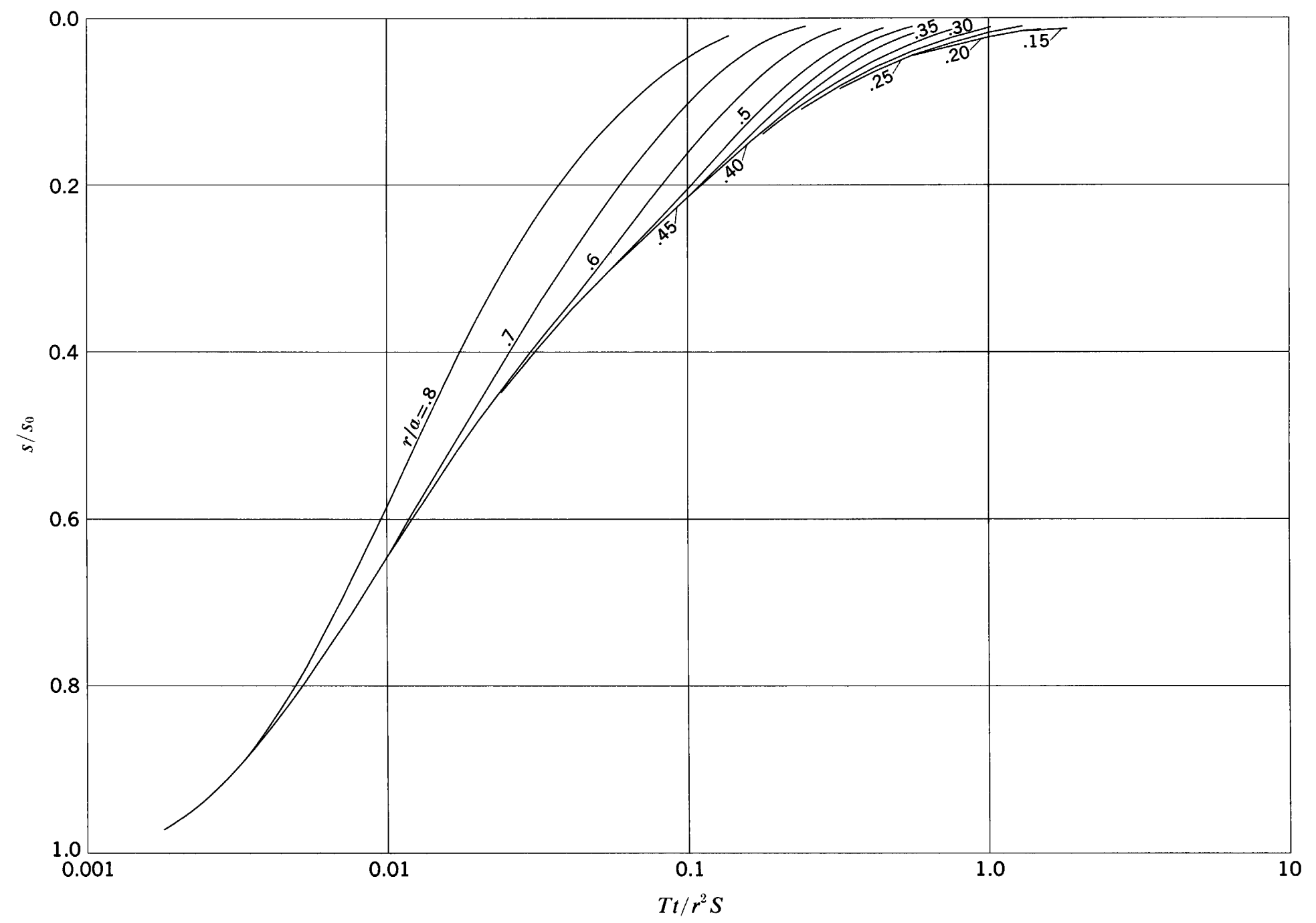

$s / s_{0}$ versus $T t / r^{2} S$ for $\theta_{0}=75^{\circ} ; \theta / \theta_{0}=0.10$ 
UNITED STATES DEPARTMENT OF THE INTERIOR GEOLOGICAL SURVEY

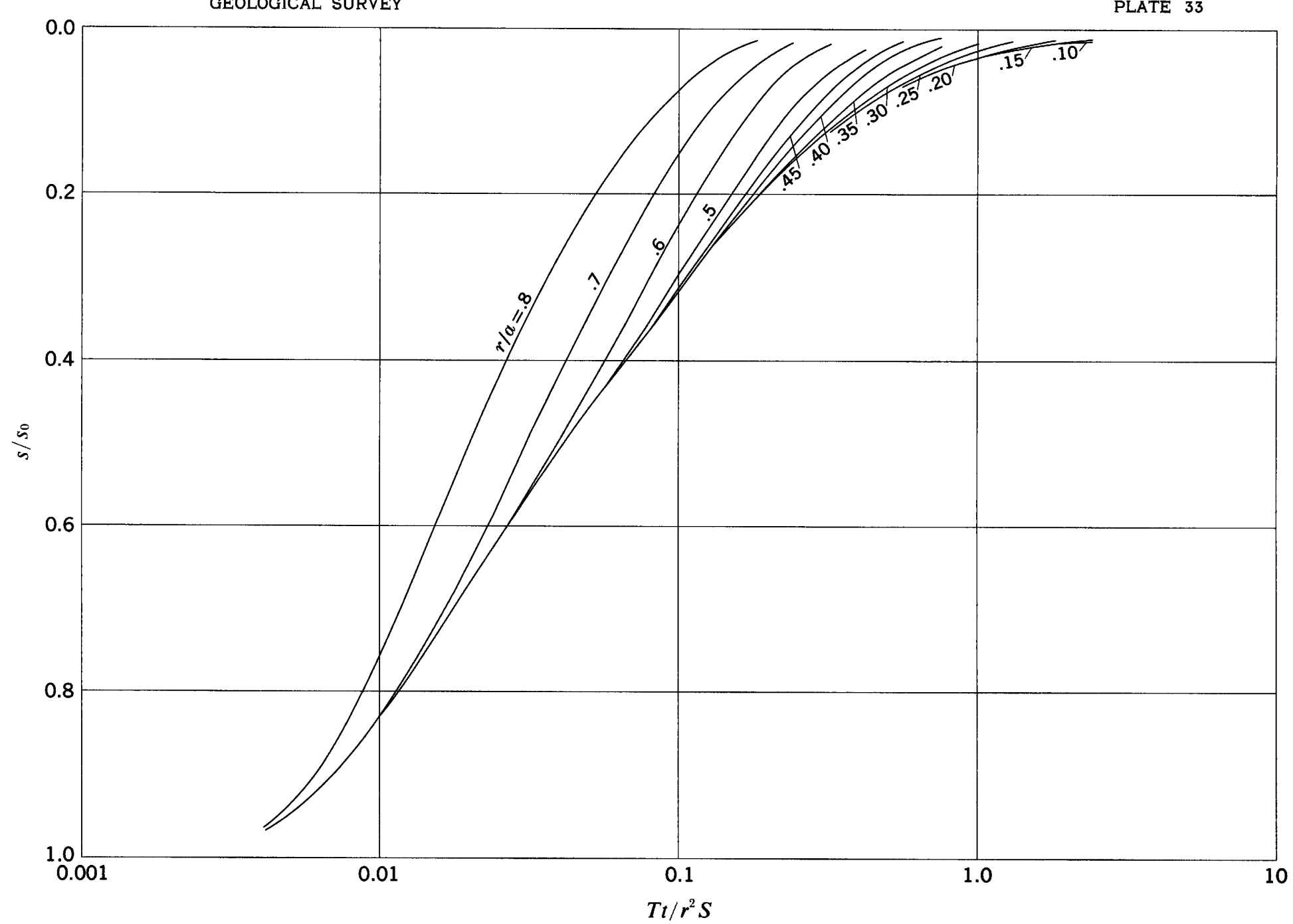

$s / s_{0}$ versus $T t / r^{2} S$ for $\theta_{0}=75^{\circ} ; \theta / \theta_{0}=0.15$ 

PLATE 34

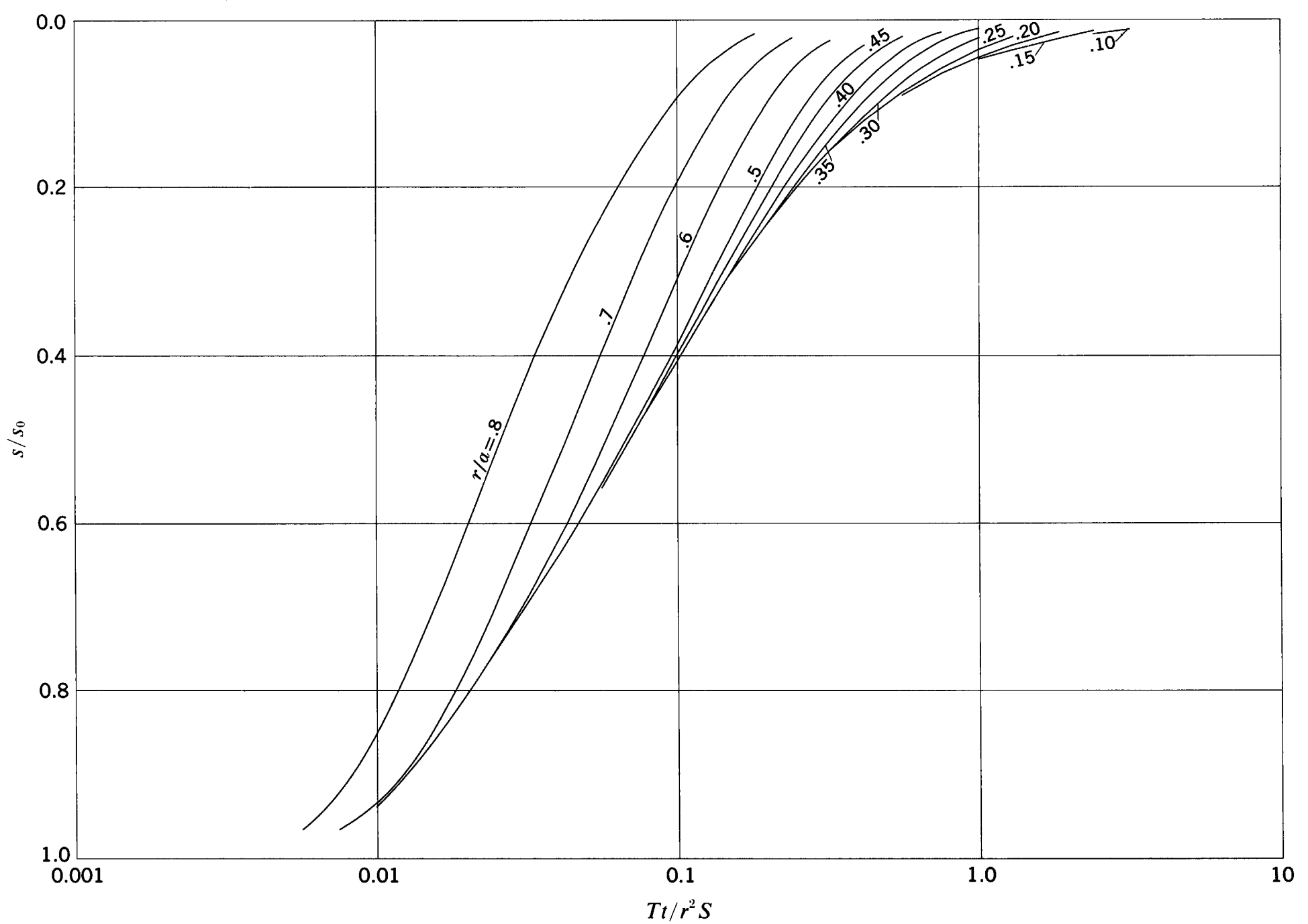

$s / s_{0}$ versus $T t / r^{2} S$ for $\theta_{0}=75^{\circ} ; \theta / \theta_{0}=0.20$ 

GEOLOGICAL SURVEY PLATE 35

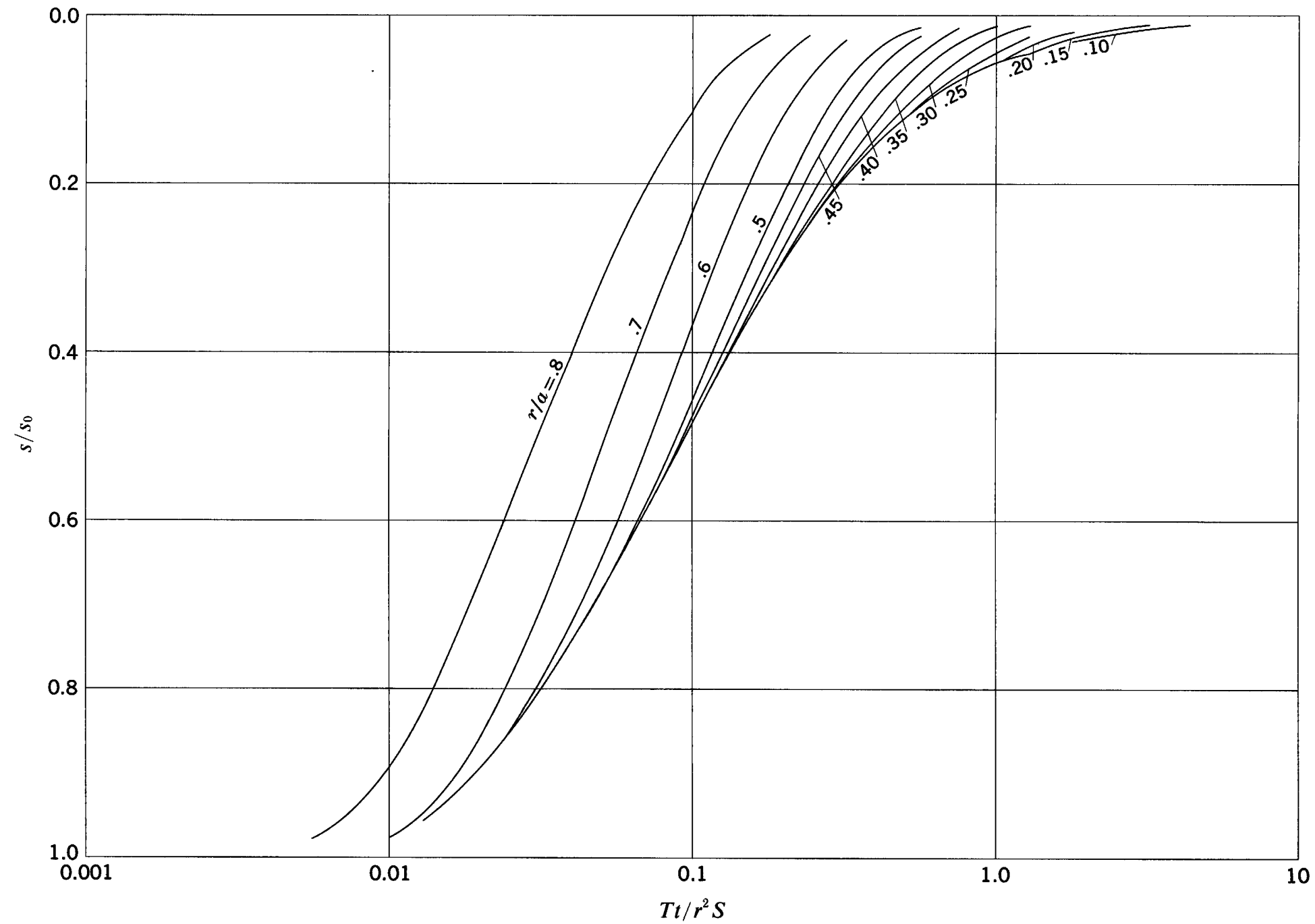

$s / s_{0}$ versus $T t / r^{2} S$ for $\theta_{0}=75^{\circ} ; \theta / \theta_{0}=0.25$ 
UNITED STATES DEPARTMENT OF THE INTERIOR GEOLOGICAL SURVEY PLATE 36

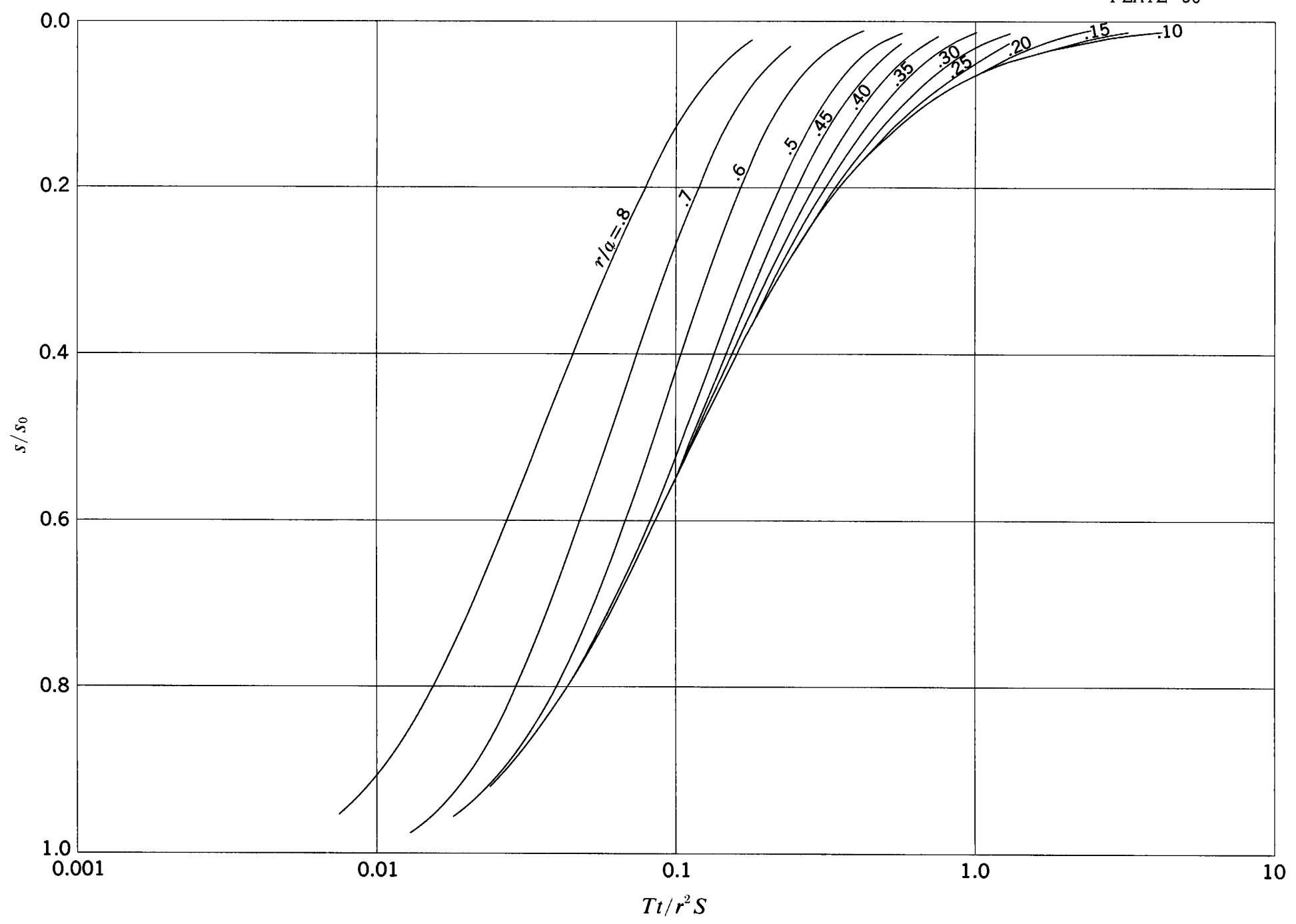

$s / s_{0}$ versus $T t / r^{2} S$ for $\theta_{0}=75^{\circ} ; \theta / \theta_{0}=0.30$ 

GEOLOGICAL SURVEY PLATE 37

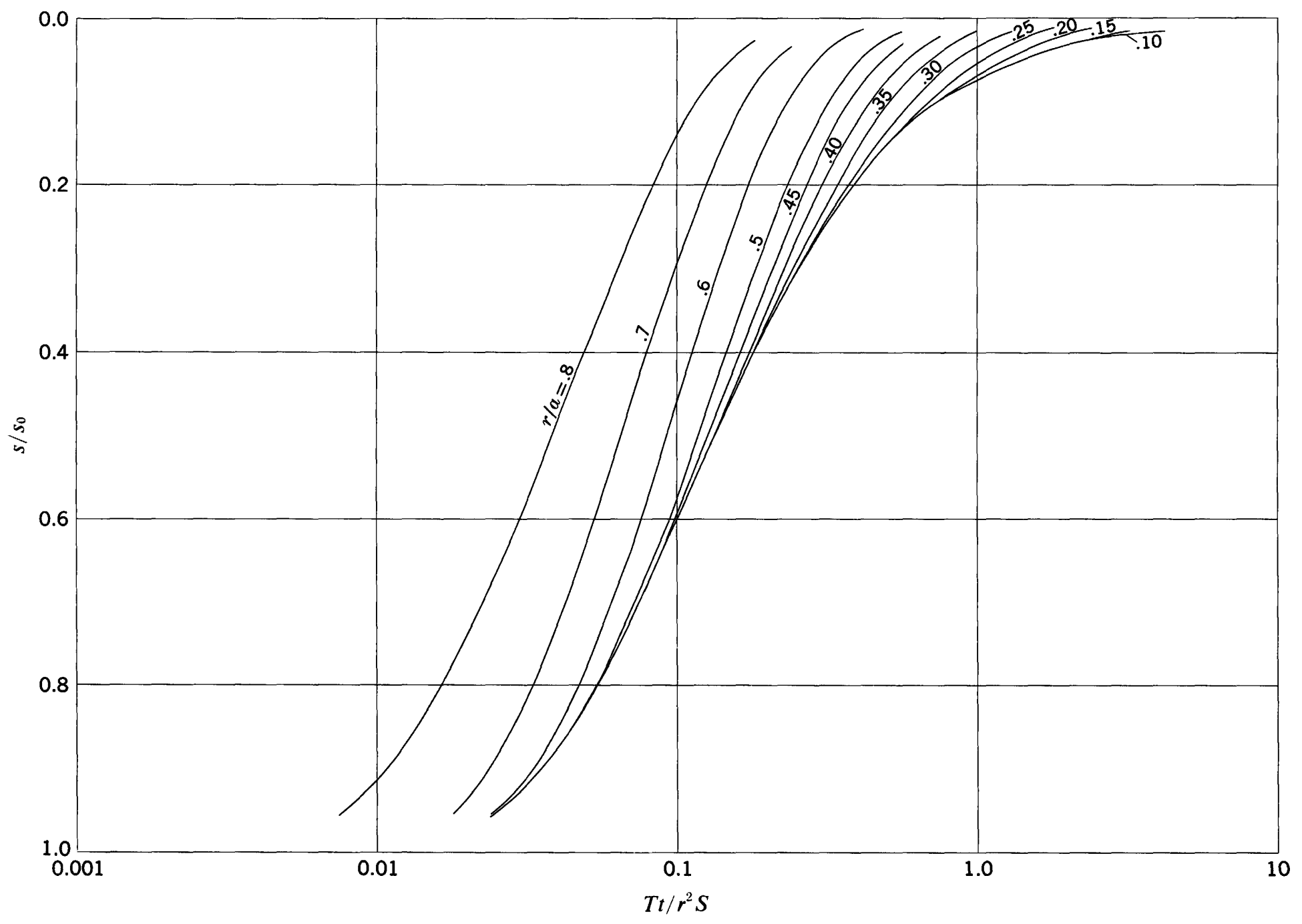

$s / s_{0}$ versus $T t / r^{2} S$ for $\theta_{0}=75^{\circ} ; \theta / \theta_{0}=0.35$ 

GEOLOGICAL SURVEY

PROFESSIONAL PAPER 514 PLATE 38

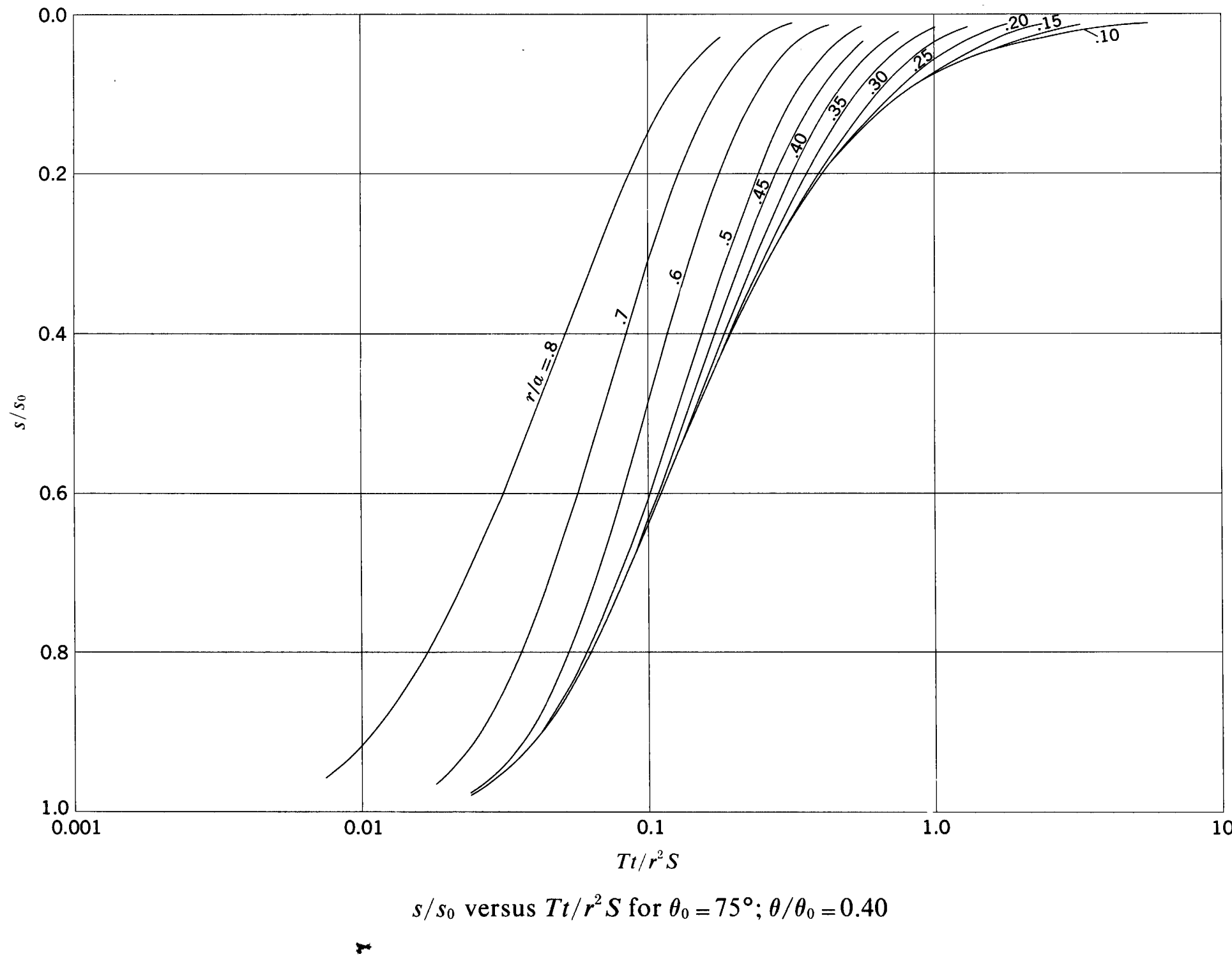




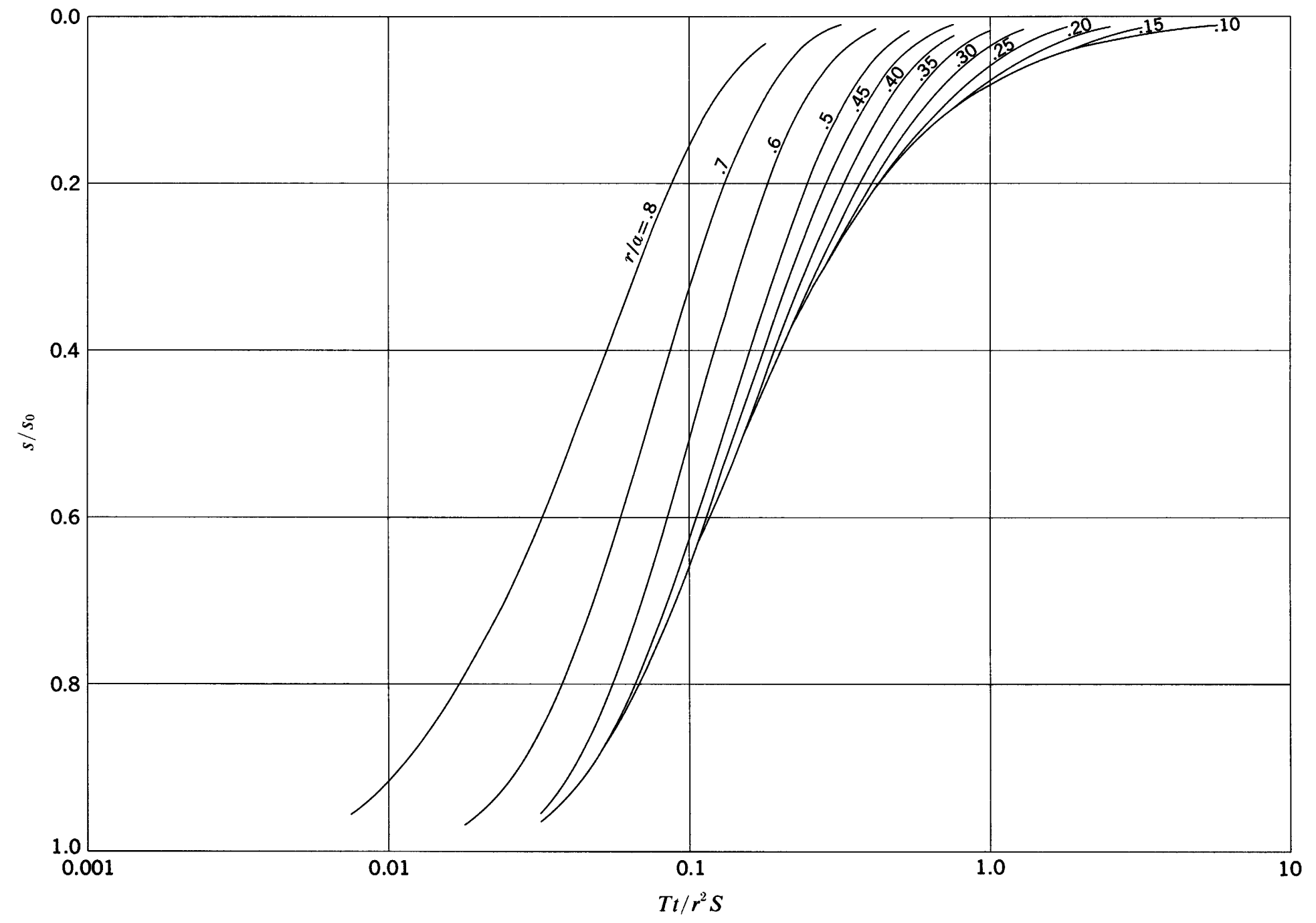

$s / s_{0}$ versus $T t / r^{2} S$ for $\theta_{0}=75^{\circ} ; \theta / \theta_{0}=0.45$ 

GEOLOGICAL SURVEY PLATE 40

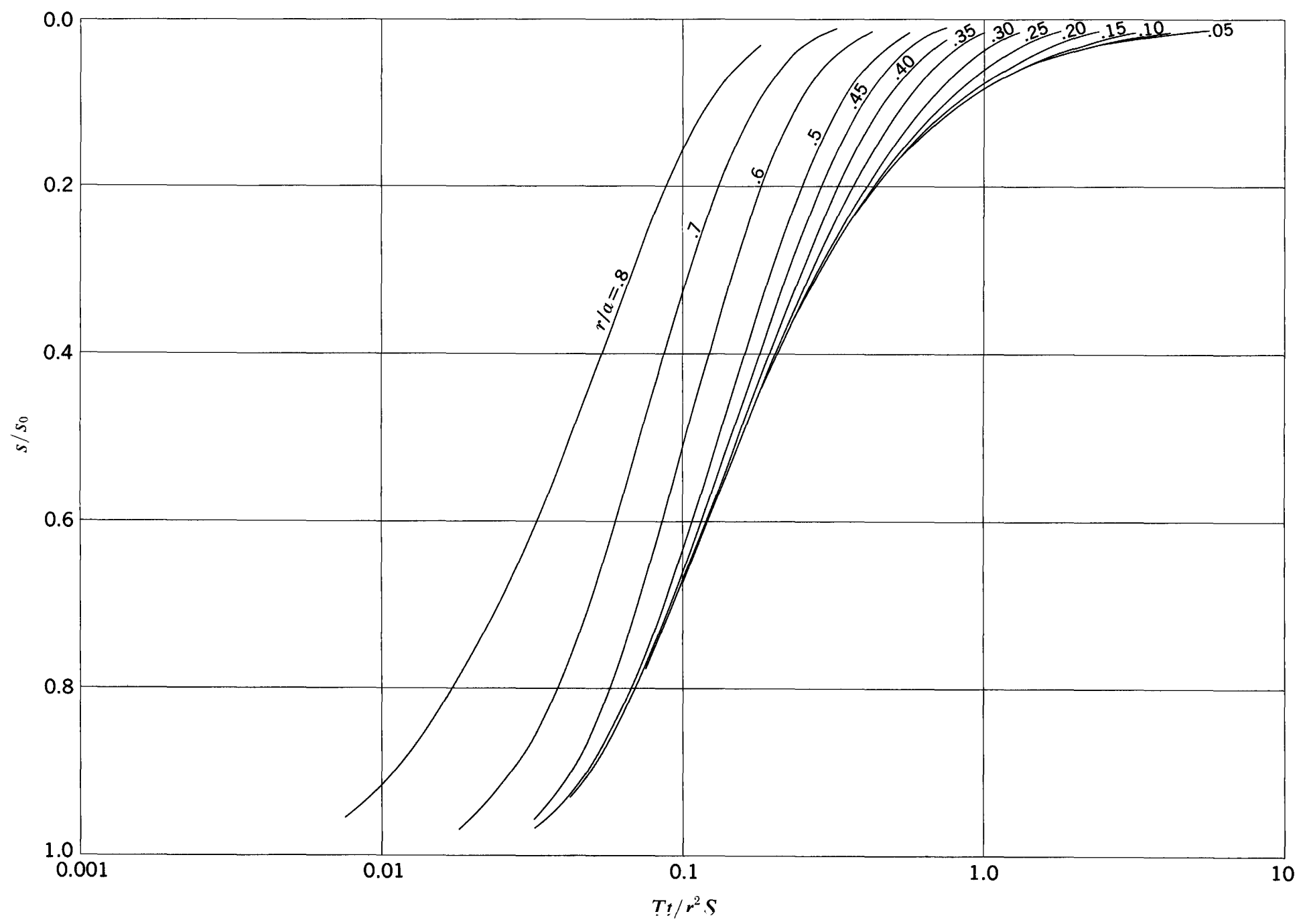

$s / s_{0}$ versus $T t / r^{2} S$ for $\theta_{0}=75^{\circ} ; \theta / \theta_{0}=0.50$ 

GEOLOGICAL SURVEY PLATE 41

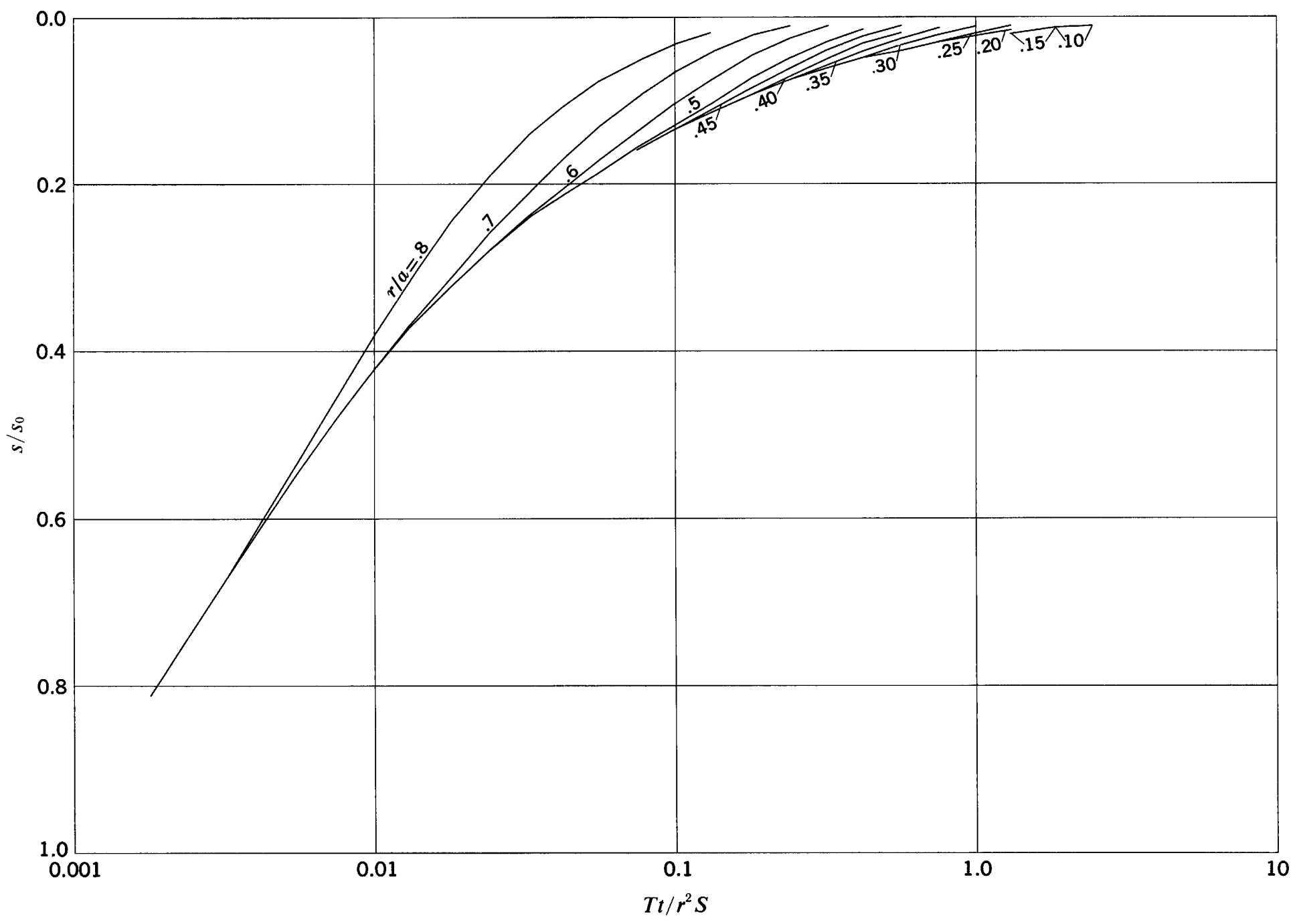

$s / s_{0}$ versus $T t / r^{2} S$ for $\theta_{0}=90^{\circ} ; \theta / \theta_{0}=0.05$ 

GEOLOGICAL SURVEY PLATE 42

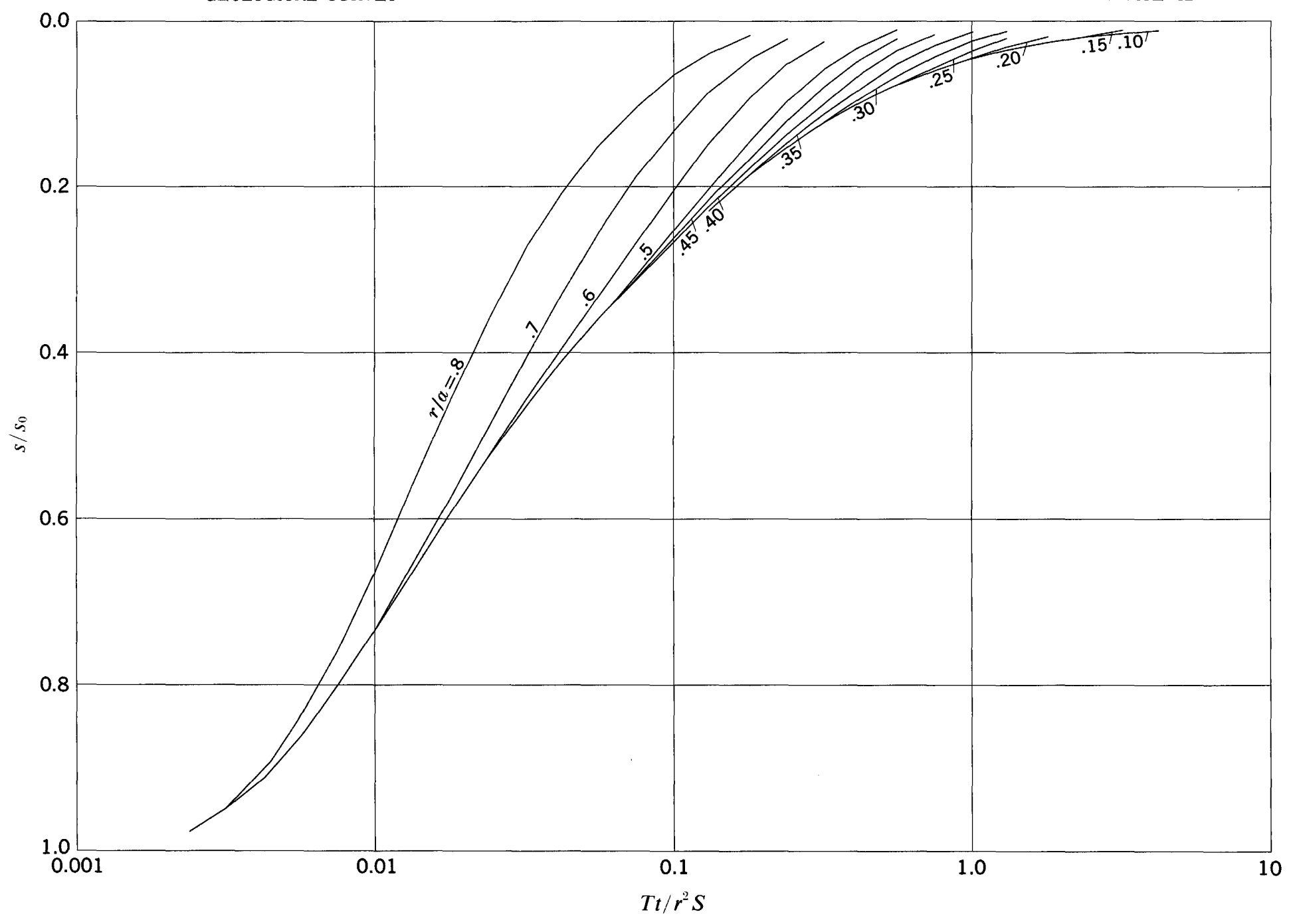

$s / s_{0}$ versus $T t / r^{2} S$ for $\theta_{0}=90^{\circ} ; \theta / \theta_{0}=0.10$ 
UNITED STATES DEPARTMENT OF THE INTERIOR GEOLOGICAL SURVEY
PROFESSIONAL PAPER 514 PLATE 43

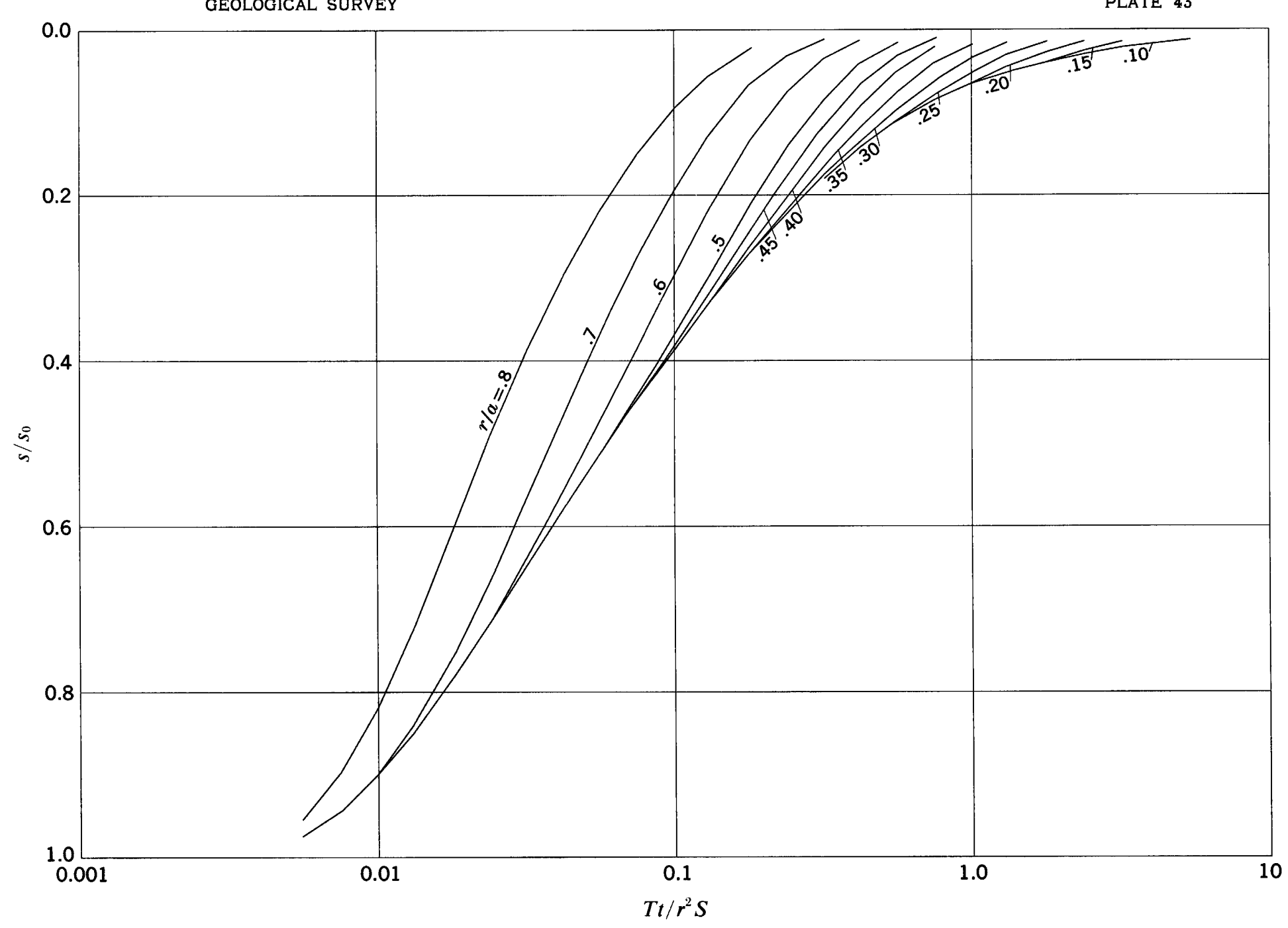

$s / s_{0}$ versus $T t / r^{2} S$ for $\theta_{0}=90^{\circ} ; \theta / \theta_{0}=0.15$ 

GEOLOGICAL SURVEY PLATE 44

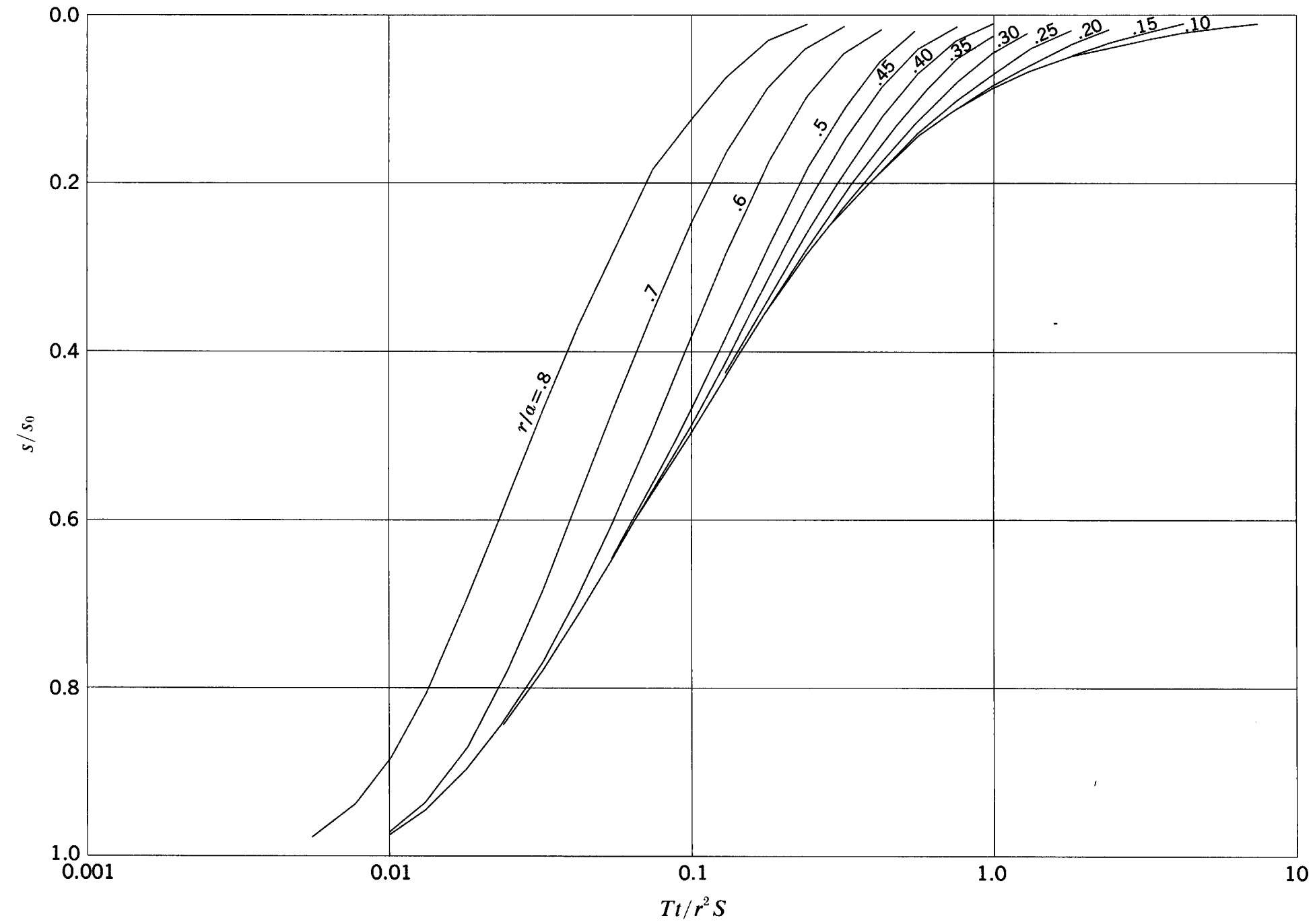

$s / s_{0}$ versus $T t / r^{2} S$ for $\theta_{0}=90^{\circ} ; \theta / \theta_{0}=0.20$ 
UNITED STATES DEPARTMENT OF THE INTERIOR GEOLOGICAL SURVEY

PROFESSIONAL PAPER 514 PLATE 45

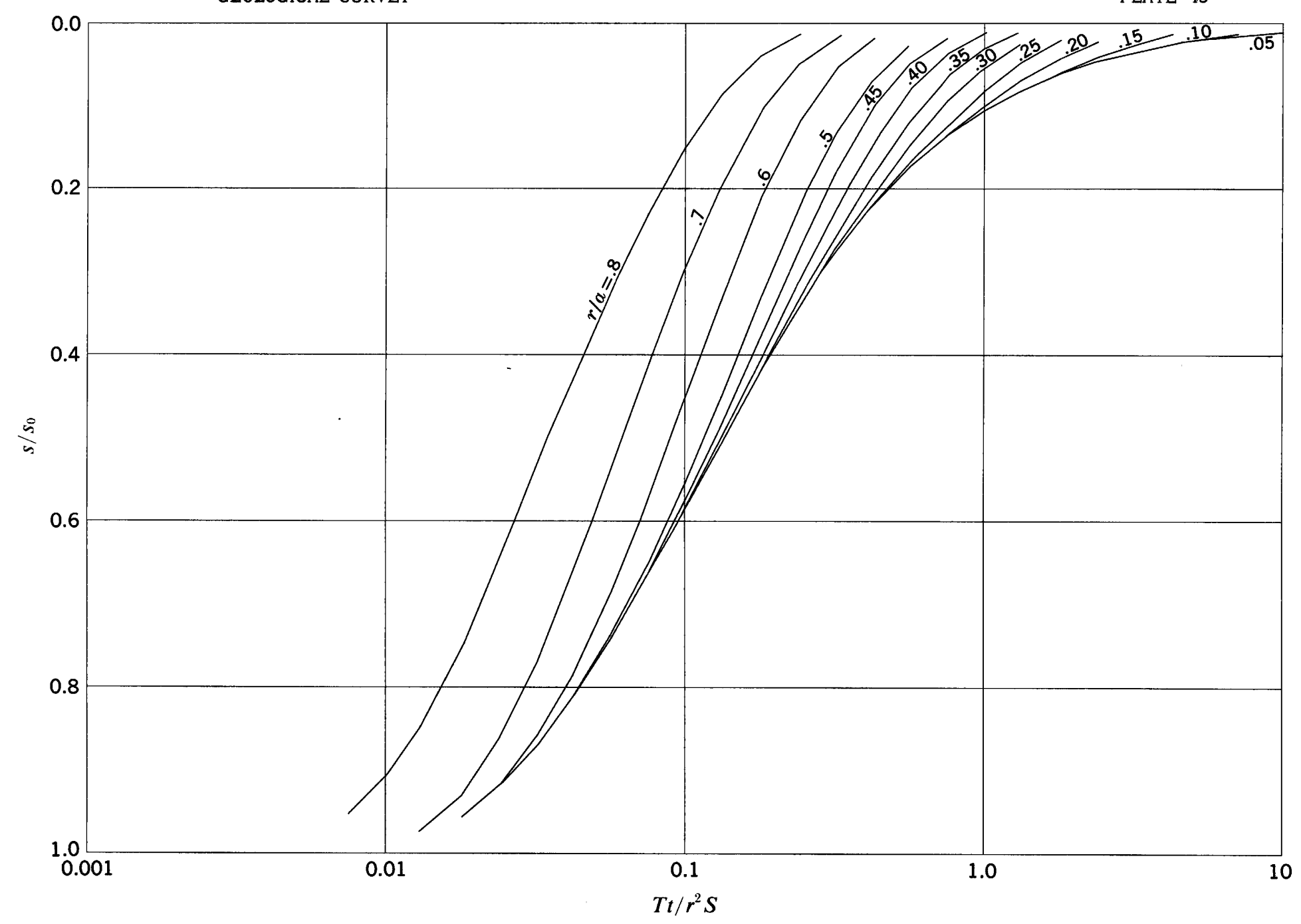

$s / s_{0}$ versus $T t / r^{2} S$ for $\theta_{0}=90^{\circ} ; \theta / \theta_{0}=0.25$ 


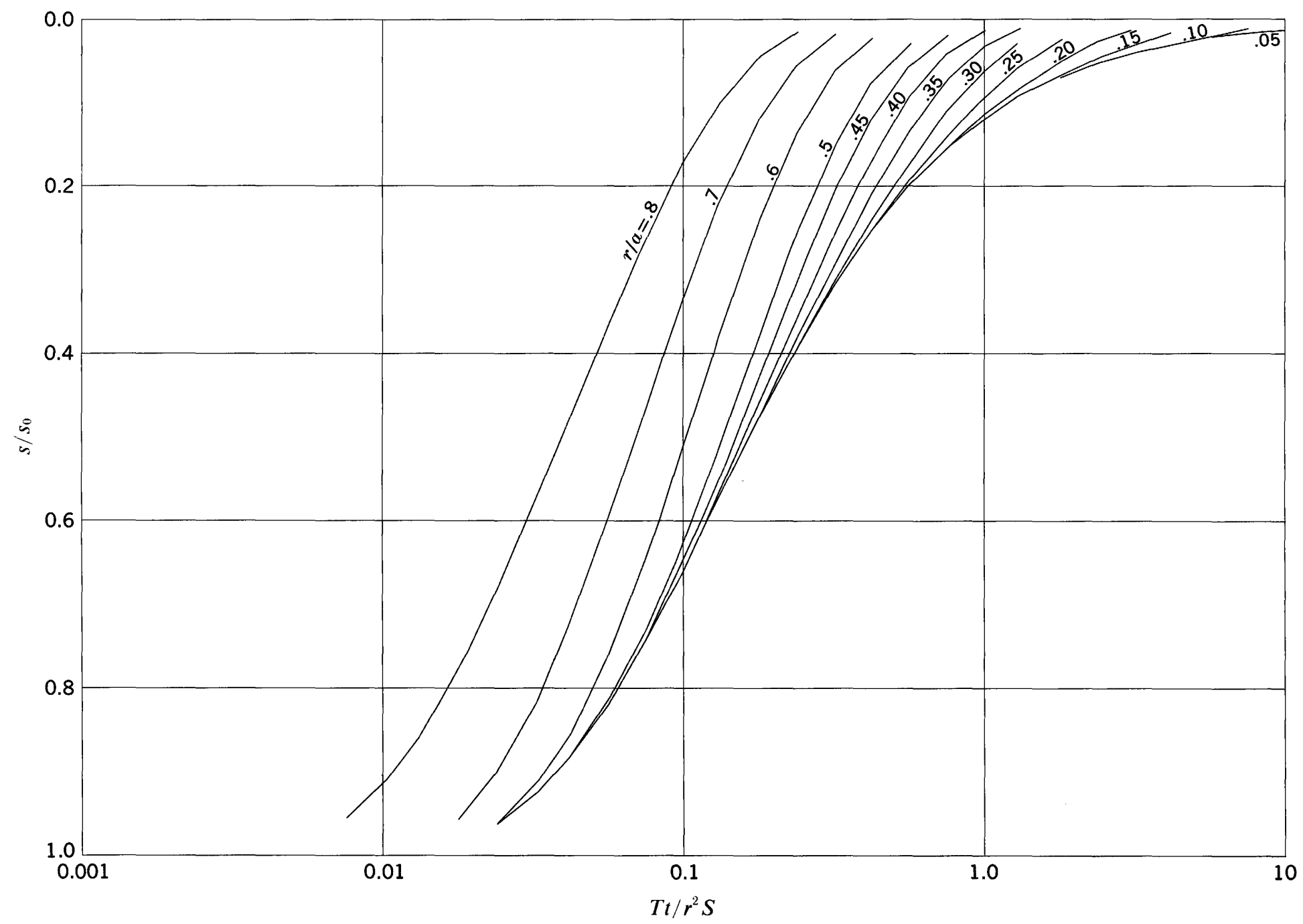

$s / s_{0}$ versus $T t / r^{2} S$ for $\theta_{0}=90^{\circ} ; \theta / \theta_{0}=0.30$ 

GEOLOGICAL SURVEY

PROFESSIONAL PAPER 514

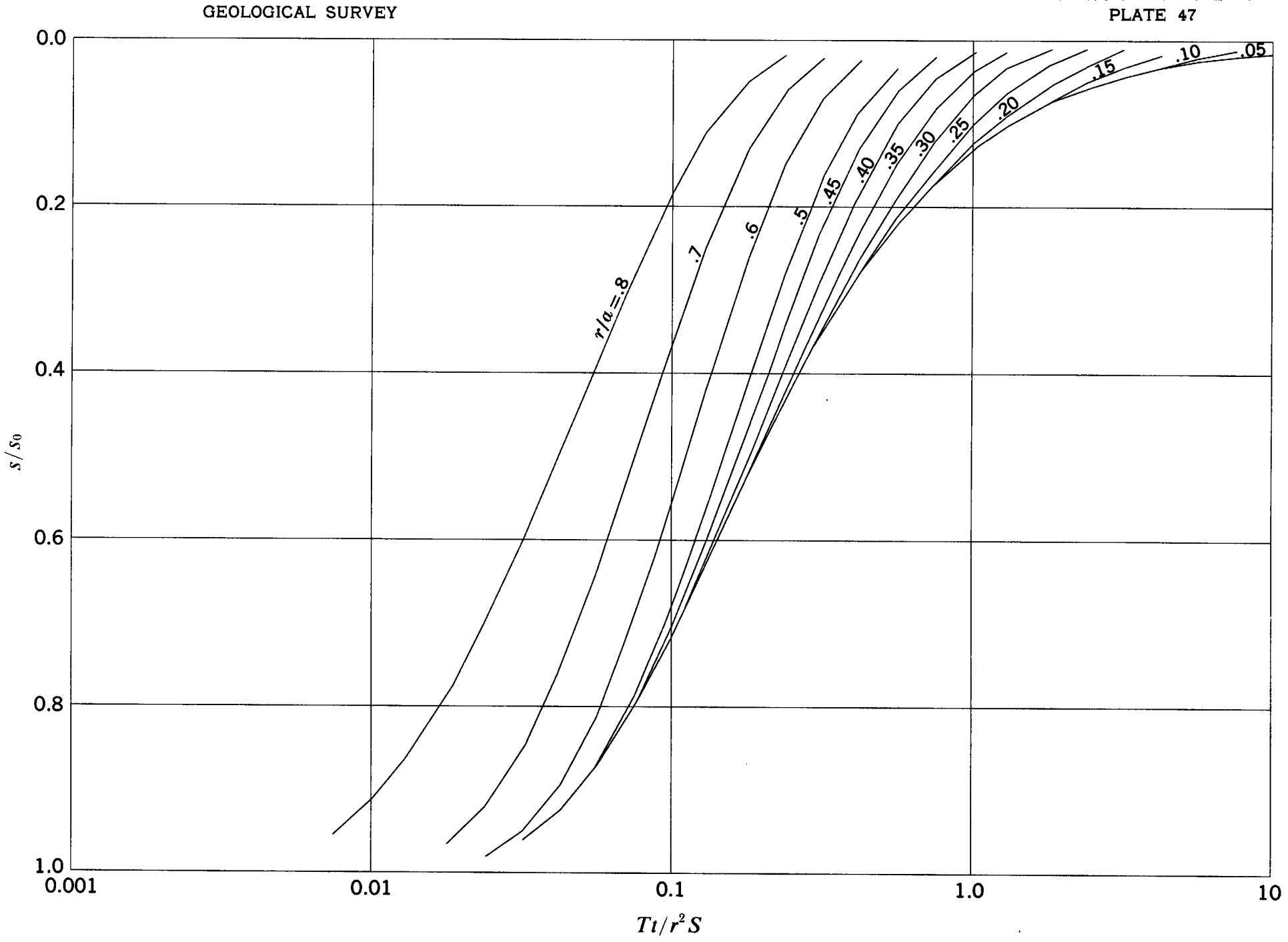

$s / s_{0}$ versus $T t / r^{2} S$ for $\theta_{0}=90^{\circ} ; \theta / \theta_{0}=0.35$ 
UNITED STATES DEPARTMENT OF THE INTERIOR GEOLOGICAL SURVEY

PROFESSIONAL PAPER 514 PLATE 48

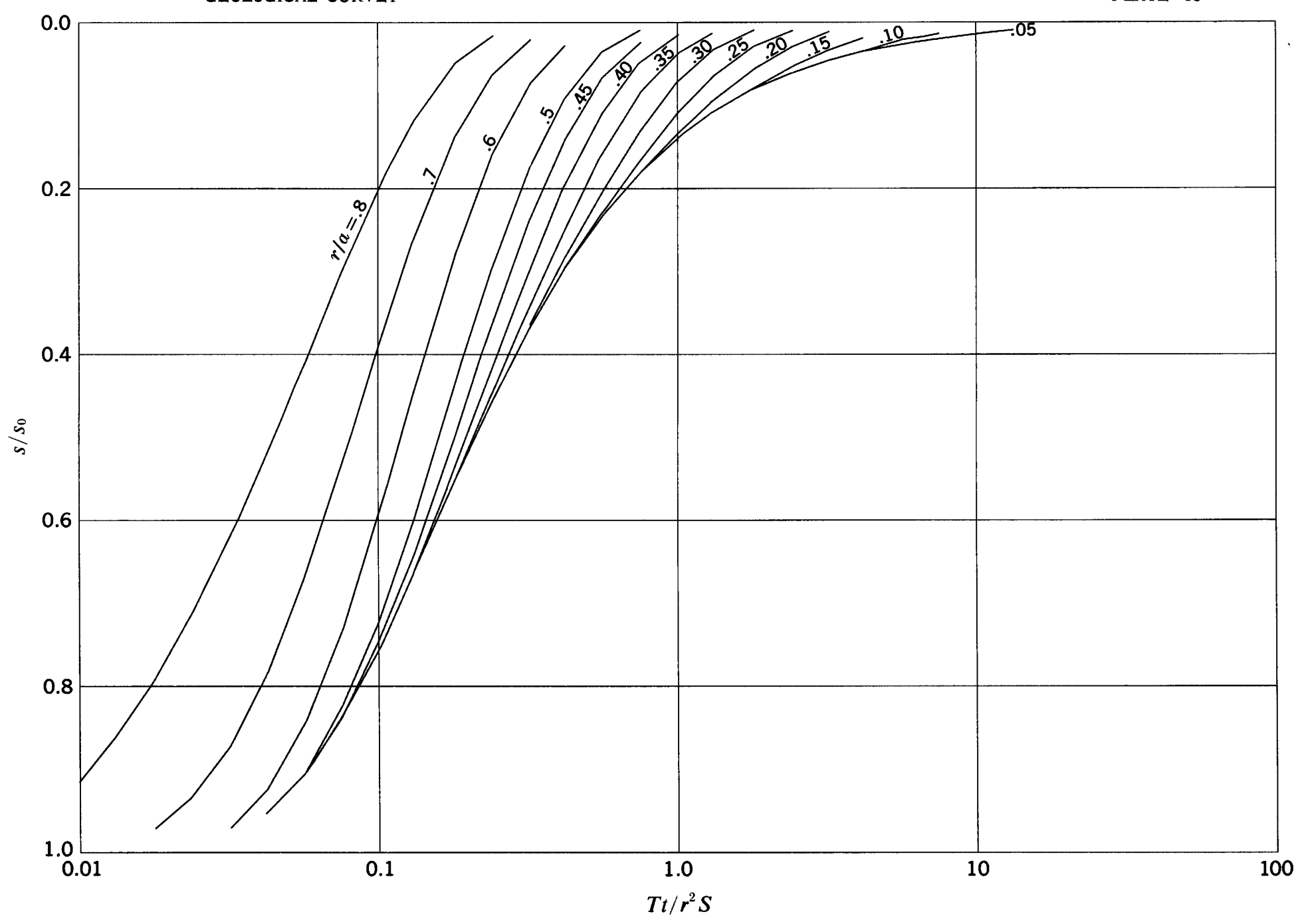

$s / s_{0}$ versus $T t / r^{2} S$ for $\theta_{0}=90^{\circ} ; \theta / \theta_{0}=0.40$ 
UNITED STATES DEPARTMENT OF THE INTERIOR GEOLOGICAL SURVEY
PROFESSIONAL PAPER 514 PLATE 49

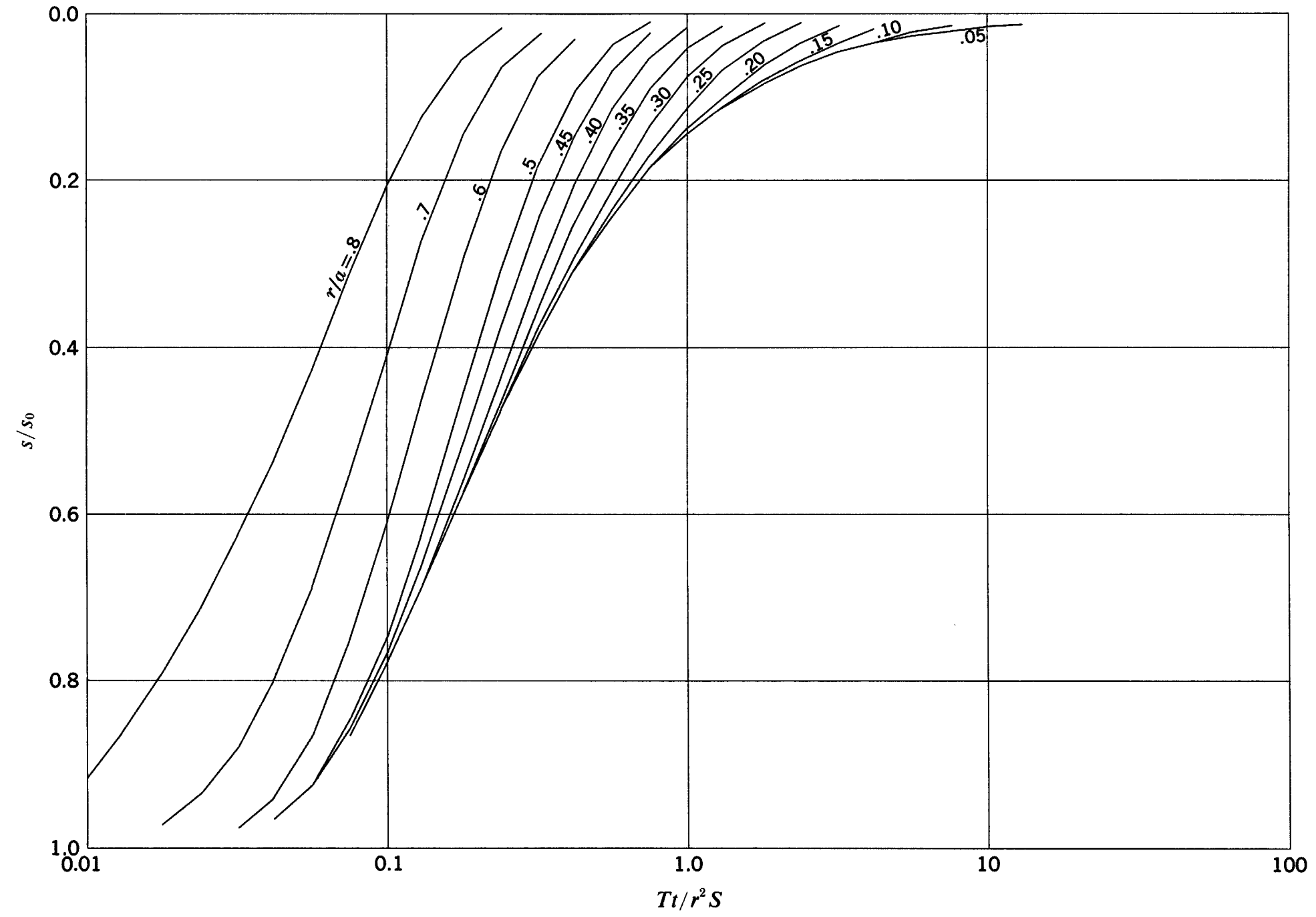

$s / s_{0}$ versus $T t / r^{2} S$ for $\theta_{0}=90^{\circ} ; \theta / \theta_{0}=0.45$ 

GEOLOGICAL SURVEY

PROFESSIONAL PAPER 514 PLATE 50

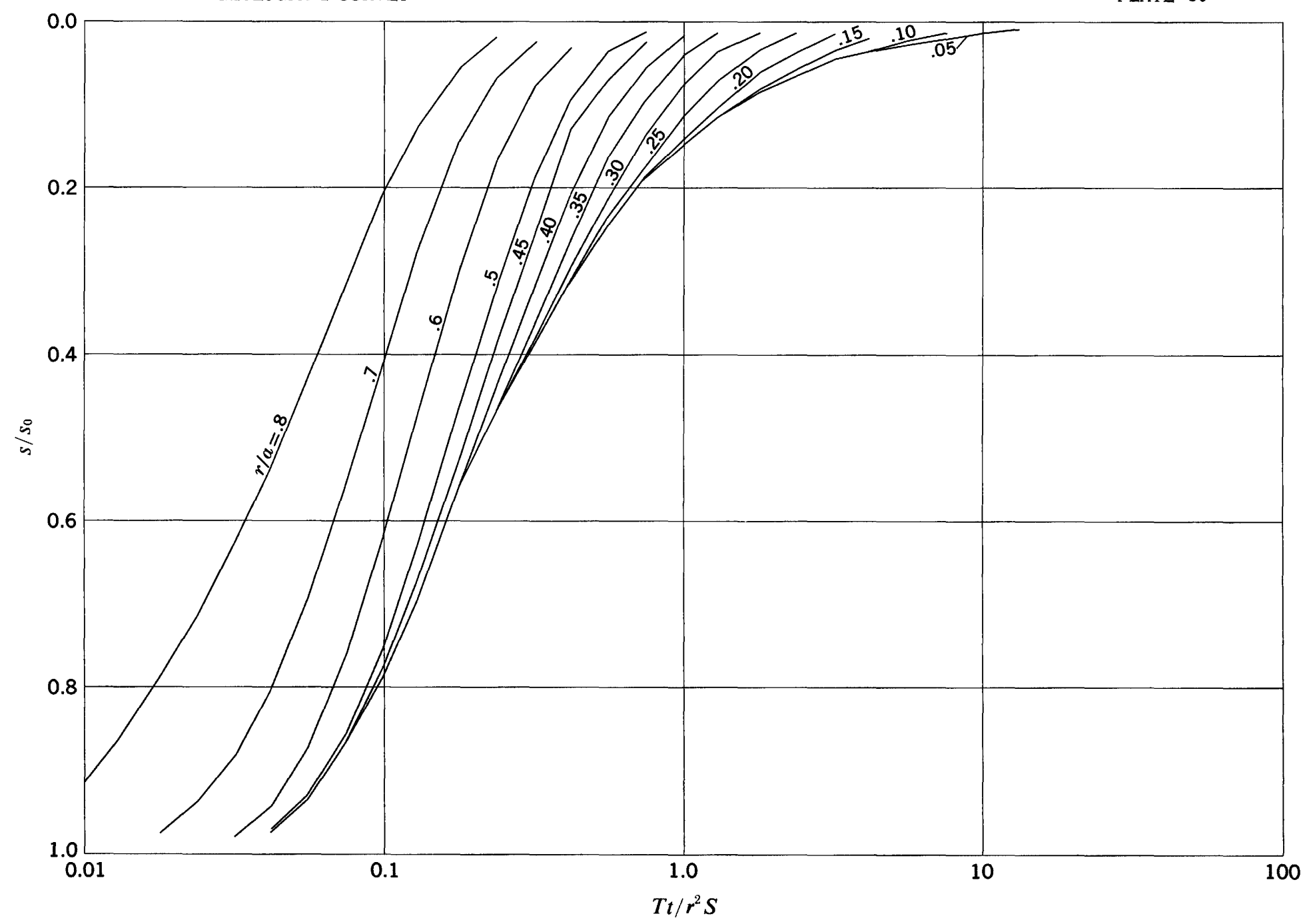

$s / s_{0}$ versus $T t / r^{2} S$ for $\theta_{0}=90^{\circ} ; \theta / \theta_{0}=0.50$ 


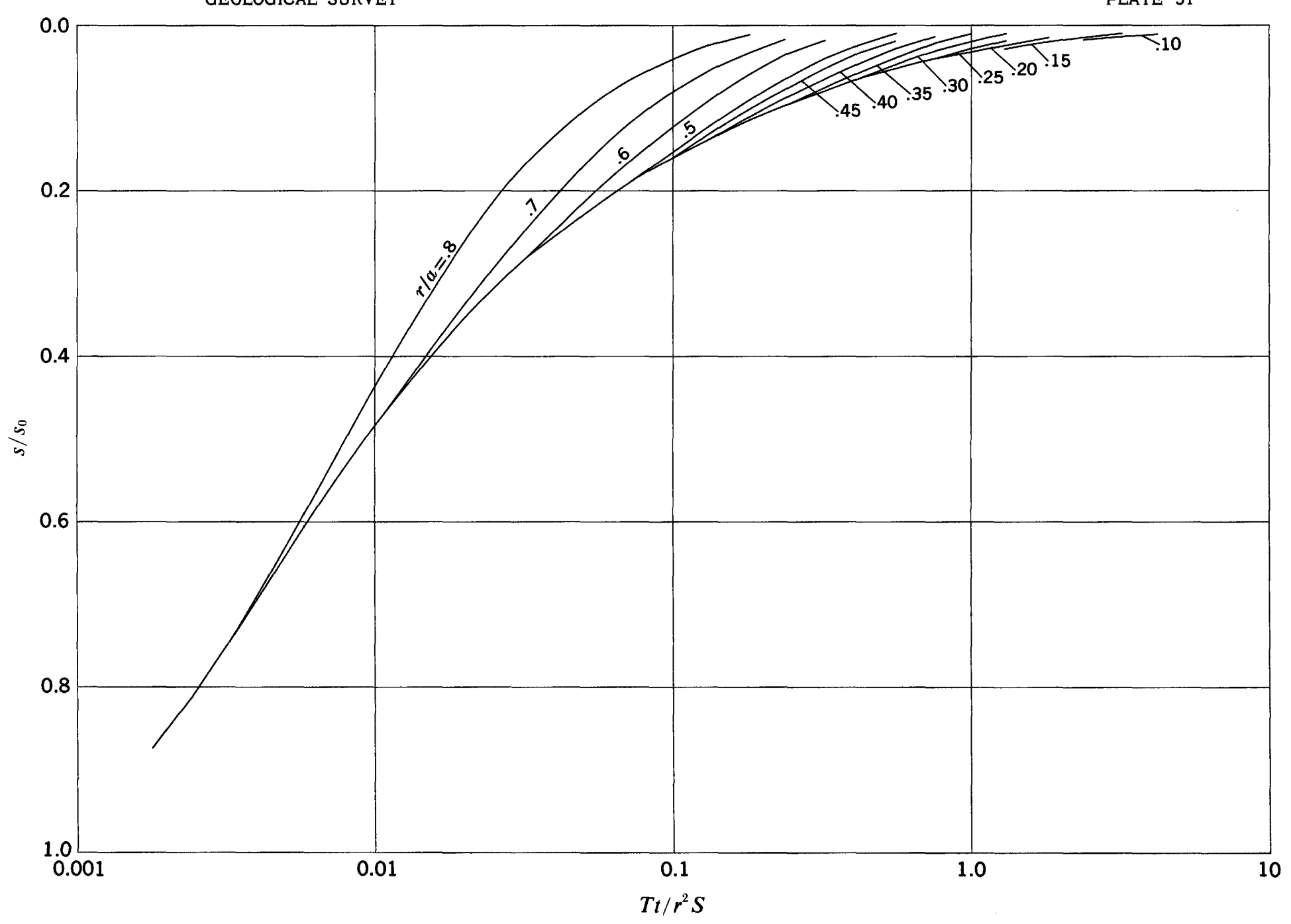

$s / s_{0}$ versus $T t / r^{2} S$ for $\theta_{0}=105^{\circ} ; \theta / \theta_{0}=0.05$ 

GEOLOGICAL SURVEY PLATE 52

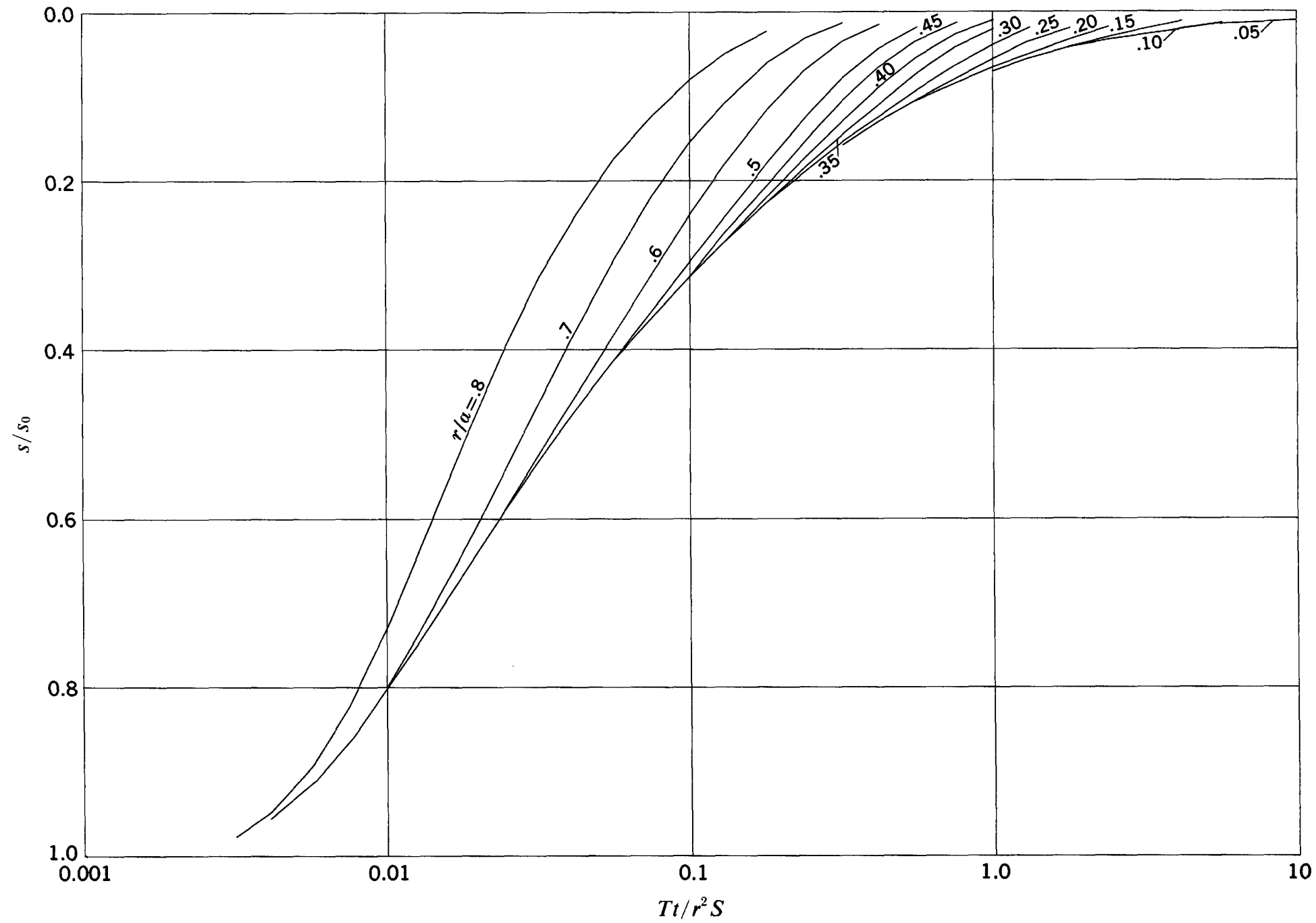

$s / s_{0}$ versus $T t / r^{2} S$ for $\theta_{0}=105^{\circ} ; \theta / \theta_{0}=0.10$ 


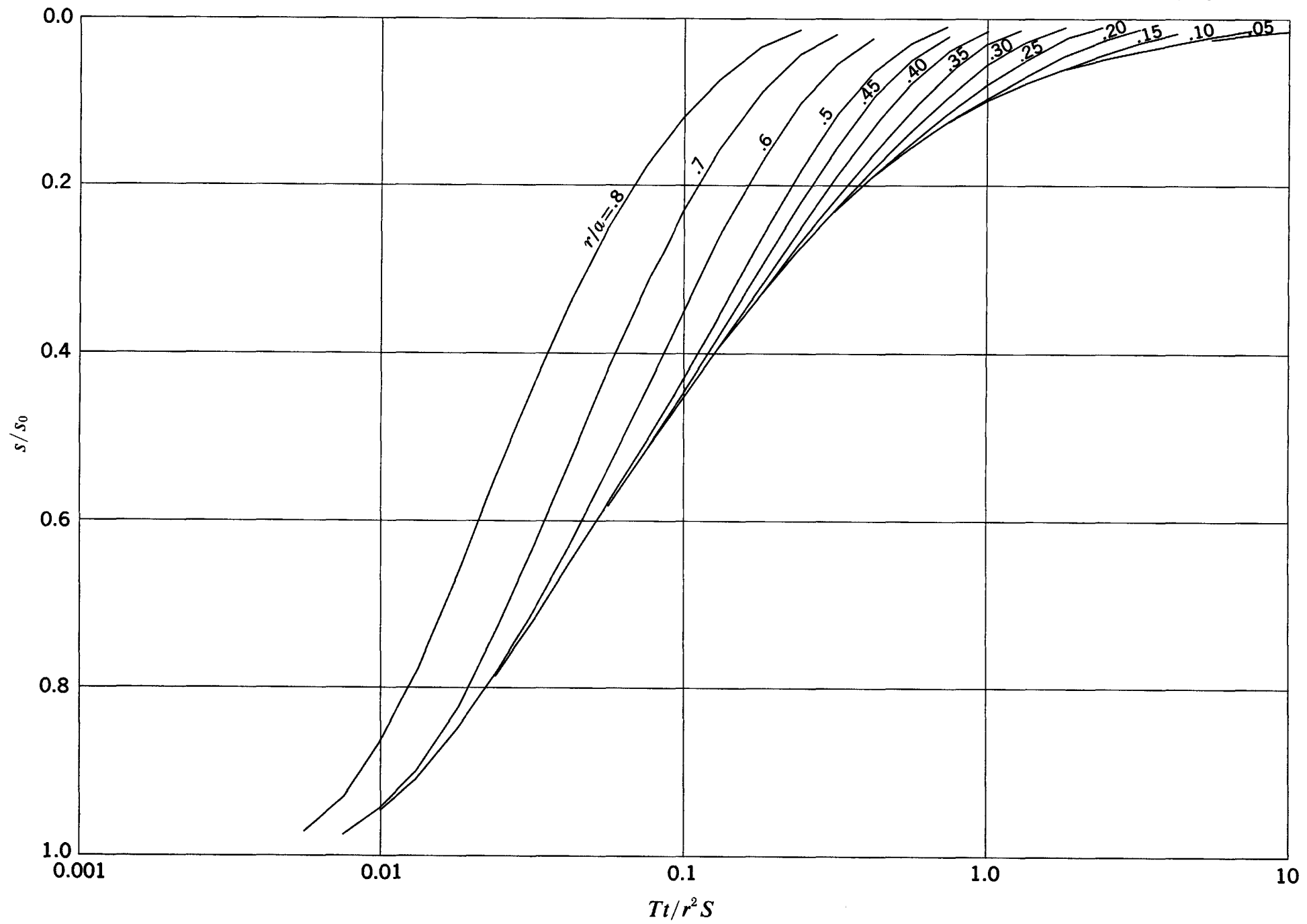

$s / s_{0}$ versus $T t / r^{2} S$ for $\theta_{0}=105^{\circ} ; \theta / \theta_{0}=0.15$ 
UNITED STATES DEPARTMENT OF THE INTERIOR

PROFESSIONAL PAPER 514 GEOLOGICAL SURVEY PLATE 54

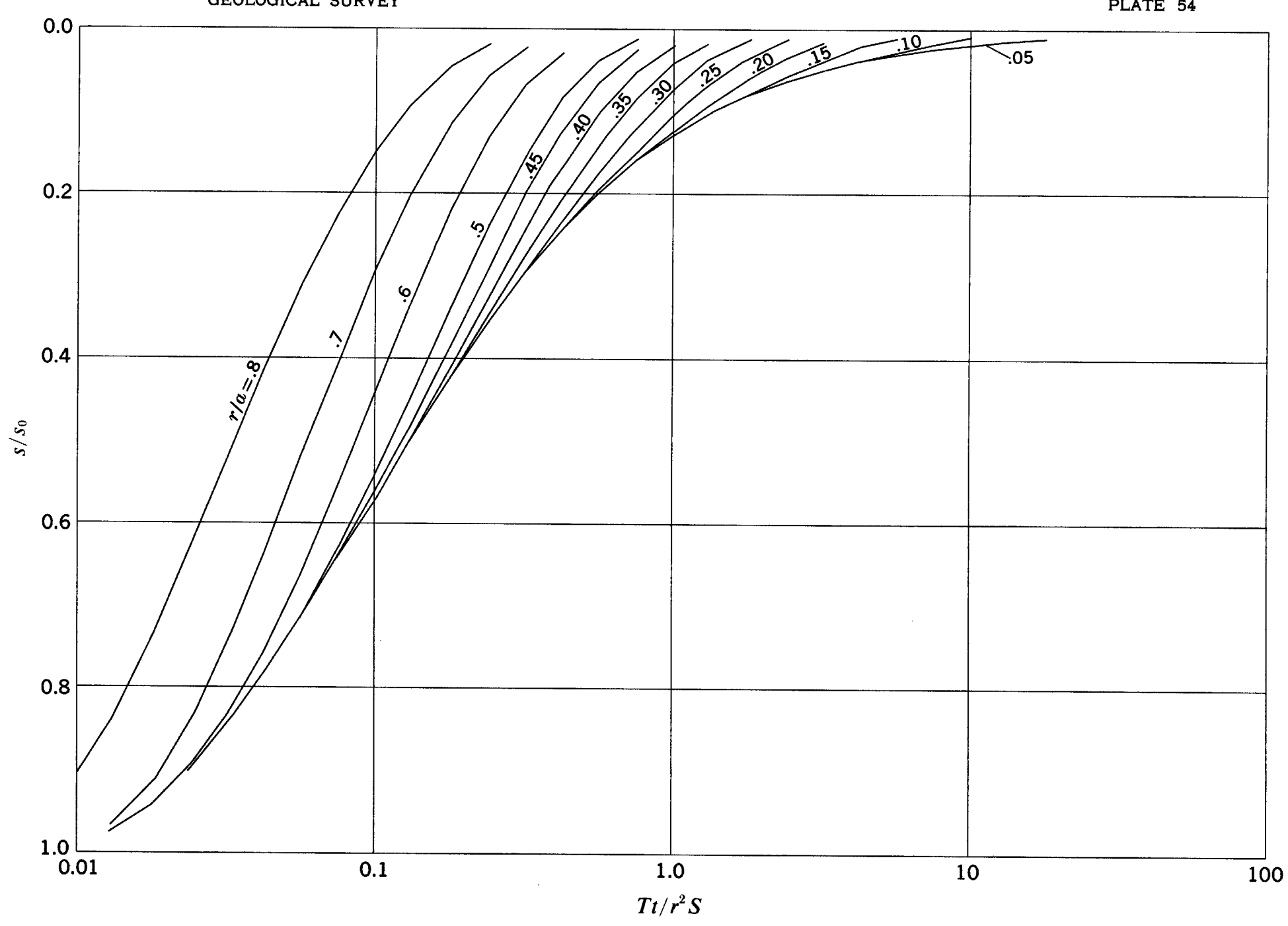

$s / s_{0}$ versus $T t / r^{2} S$ for $\theta_{0}=105^{\circ} ; \theta / \theta_{0}=0.20$ 

GEOLOGICAL SURVEY PLATE 55

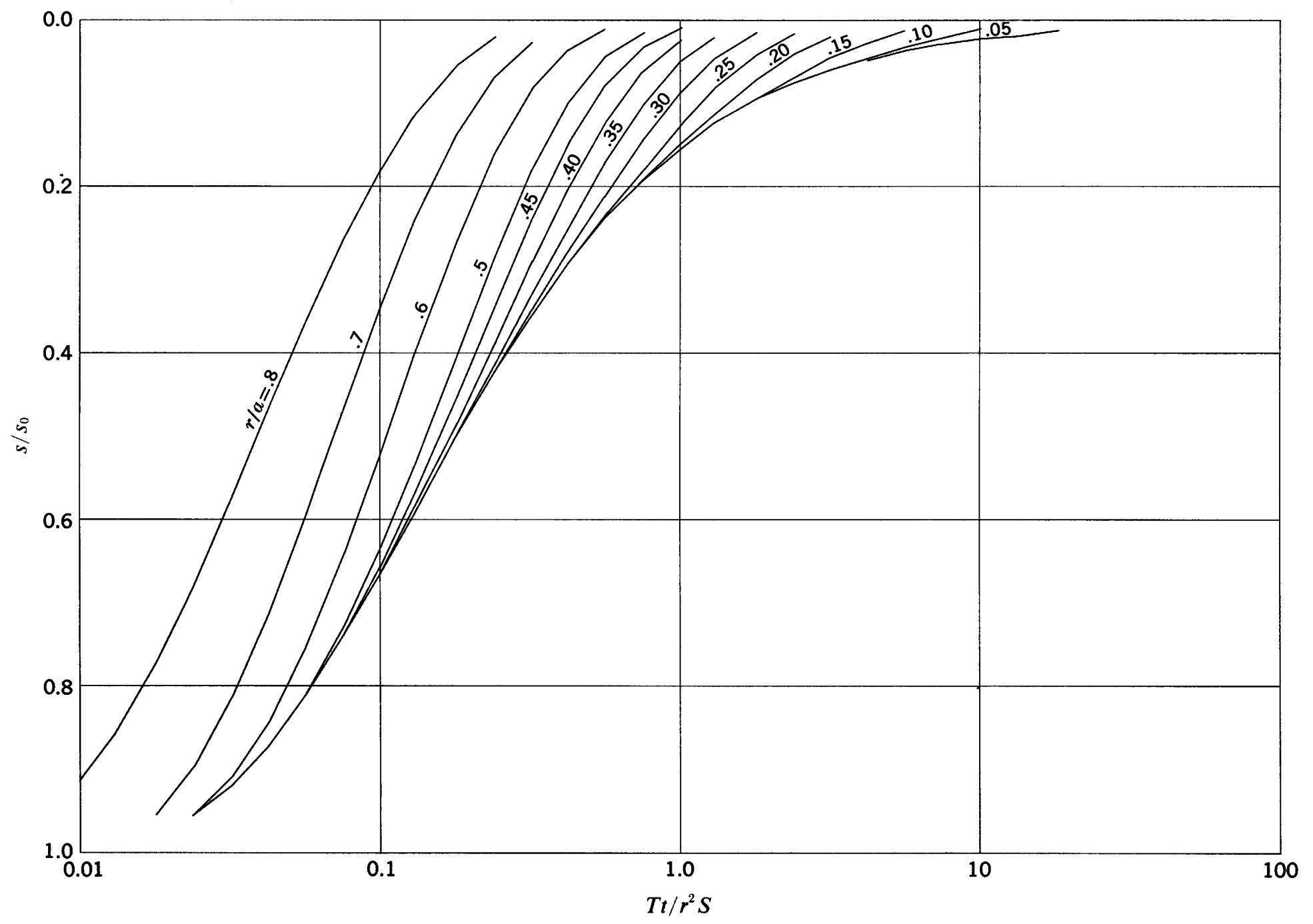

$s / s_{0}$ versus $T t / r^{2} S$ for $\theta_{0}=105^{\circ} ; \theta / \theta_{0}=0.25$ 


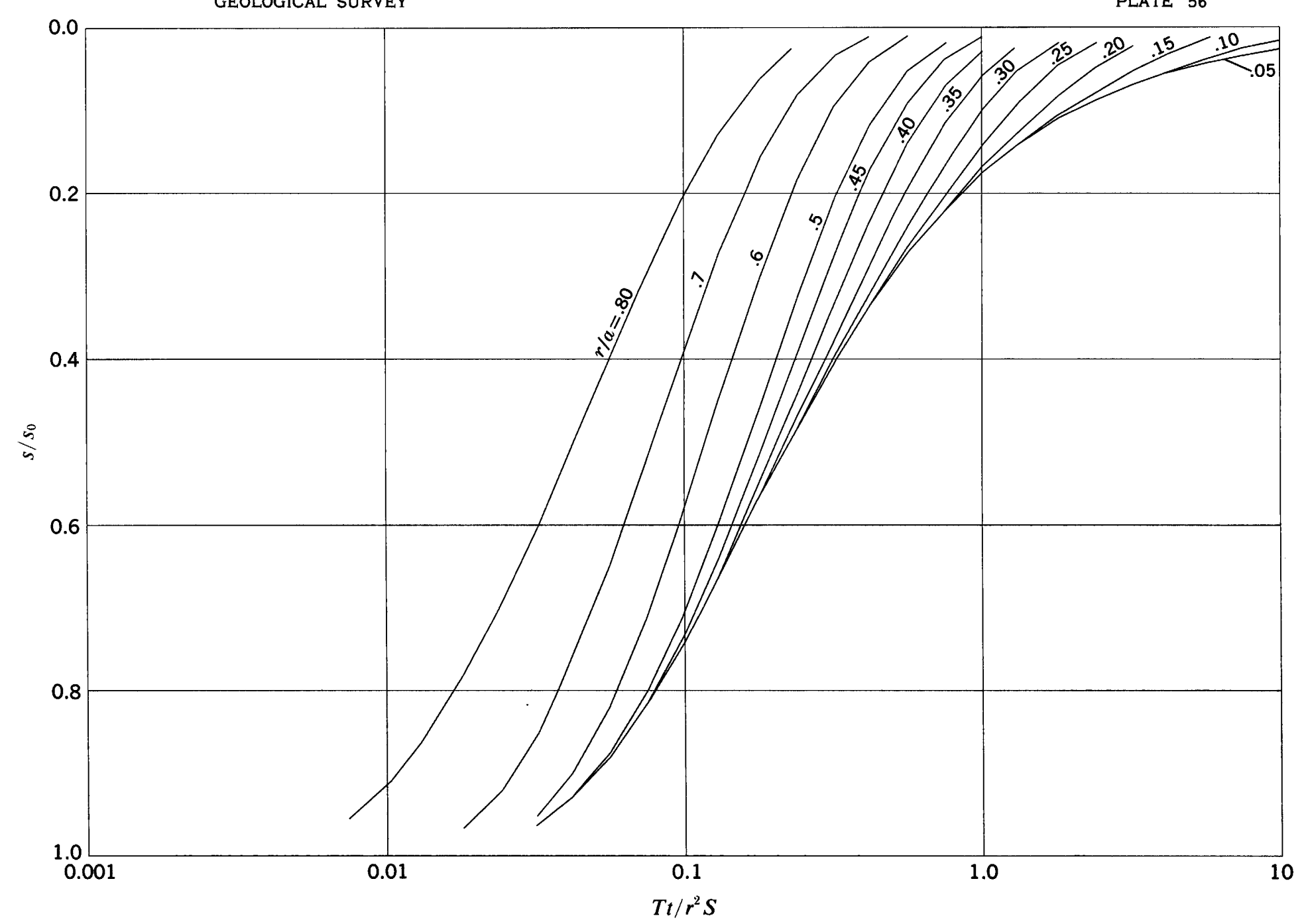

$s / s_{0}$ versus $T t / r^{2} S$ for $\theta_{0}=105^{\circ} ; \theta / \theta_{0}=0.30$ 


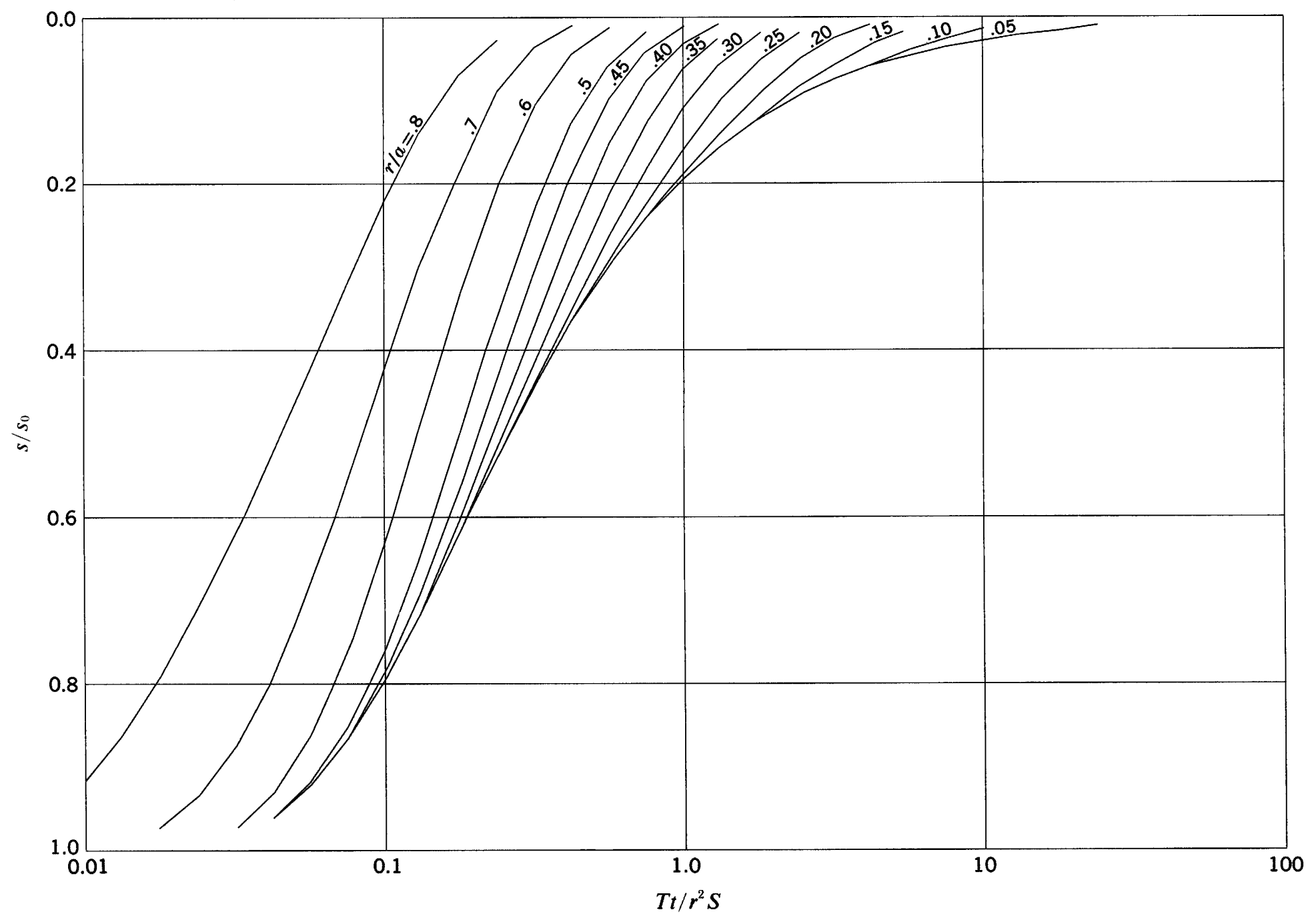

$s / s_{0}$ versus $T t / r^{2} S$ for $\theta_{0}=105^{\circ} ; \theta / \theta_{0}=0.35$ 


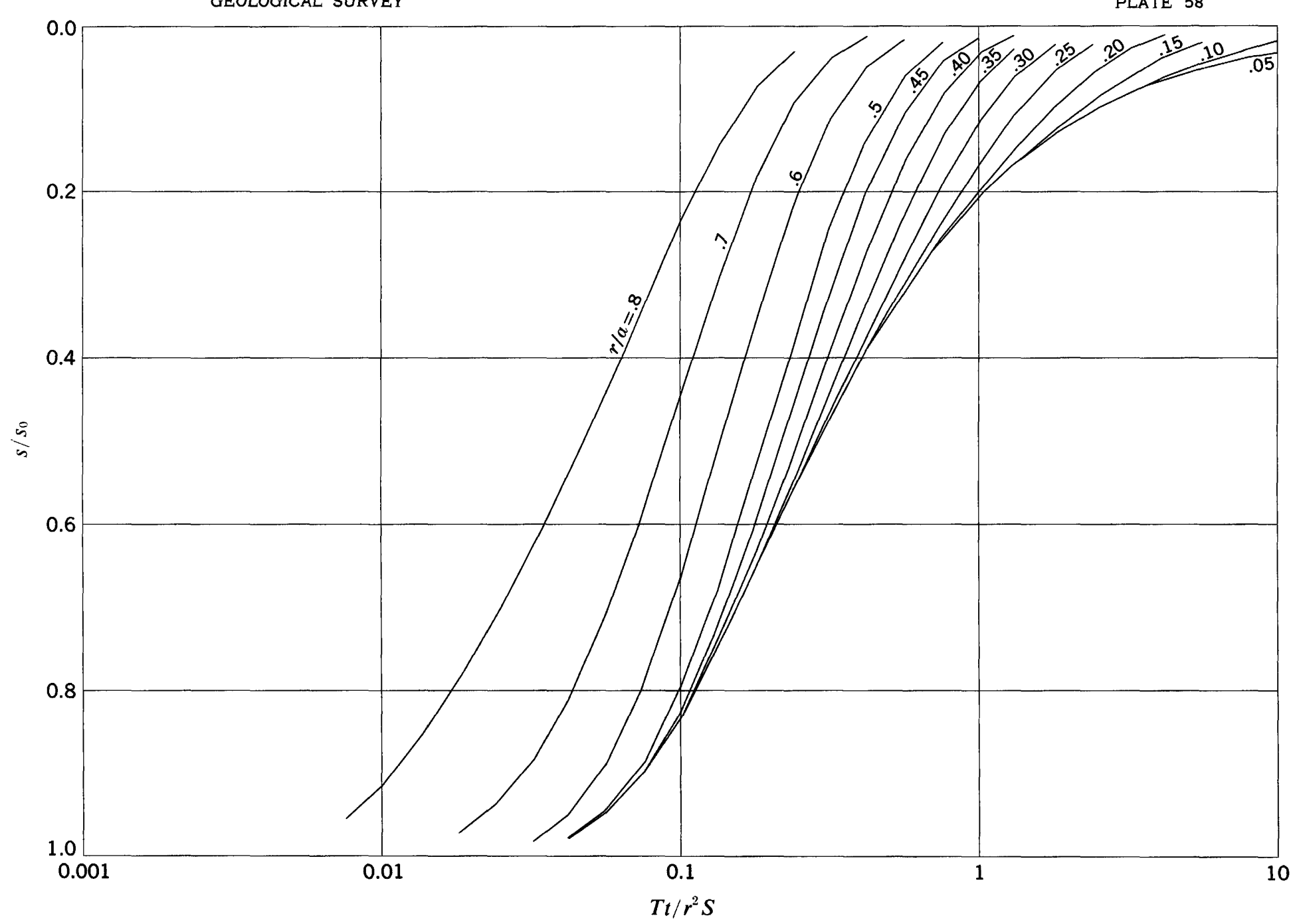

$s / s_{0}$ versus $T t / r^{2} S$ for $\theta_{0}=105^{\circ} ; \theta / \theta_{0}=0.40$ 

GEOLOGICAL SURVEY

PLATE 59

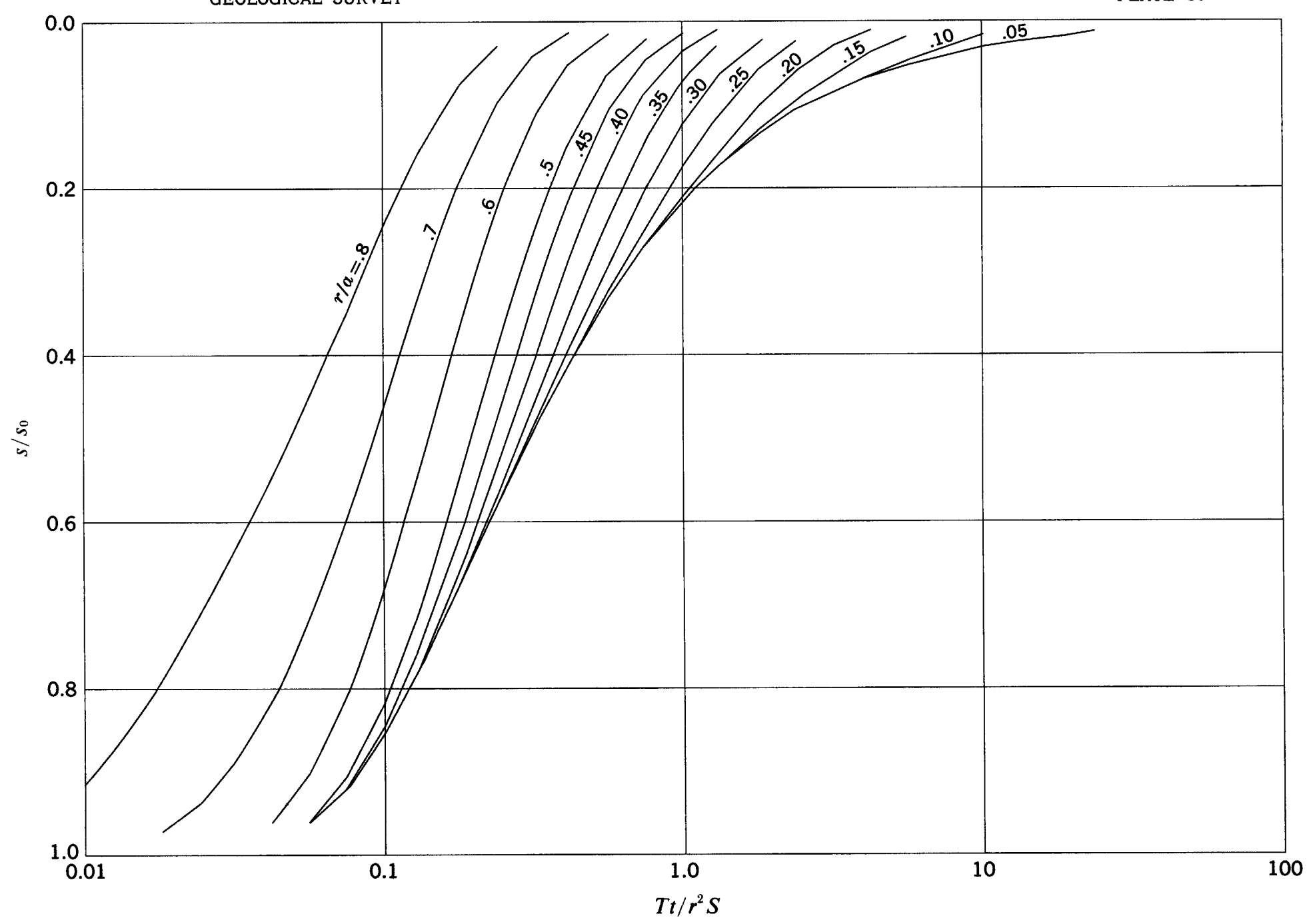

$s / s_{0}$ versus $T t / r^{2} S$ for $\theta_{0}=105^{\circ} ; \theta / \theta_{0}=0.45$ 

GEOLOGICAL SURVEY

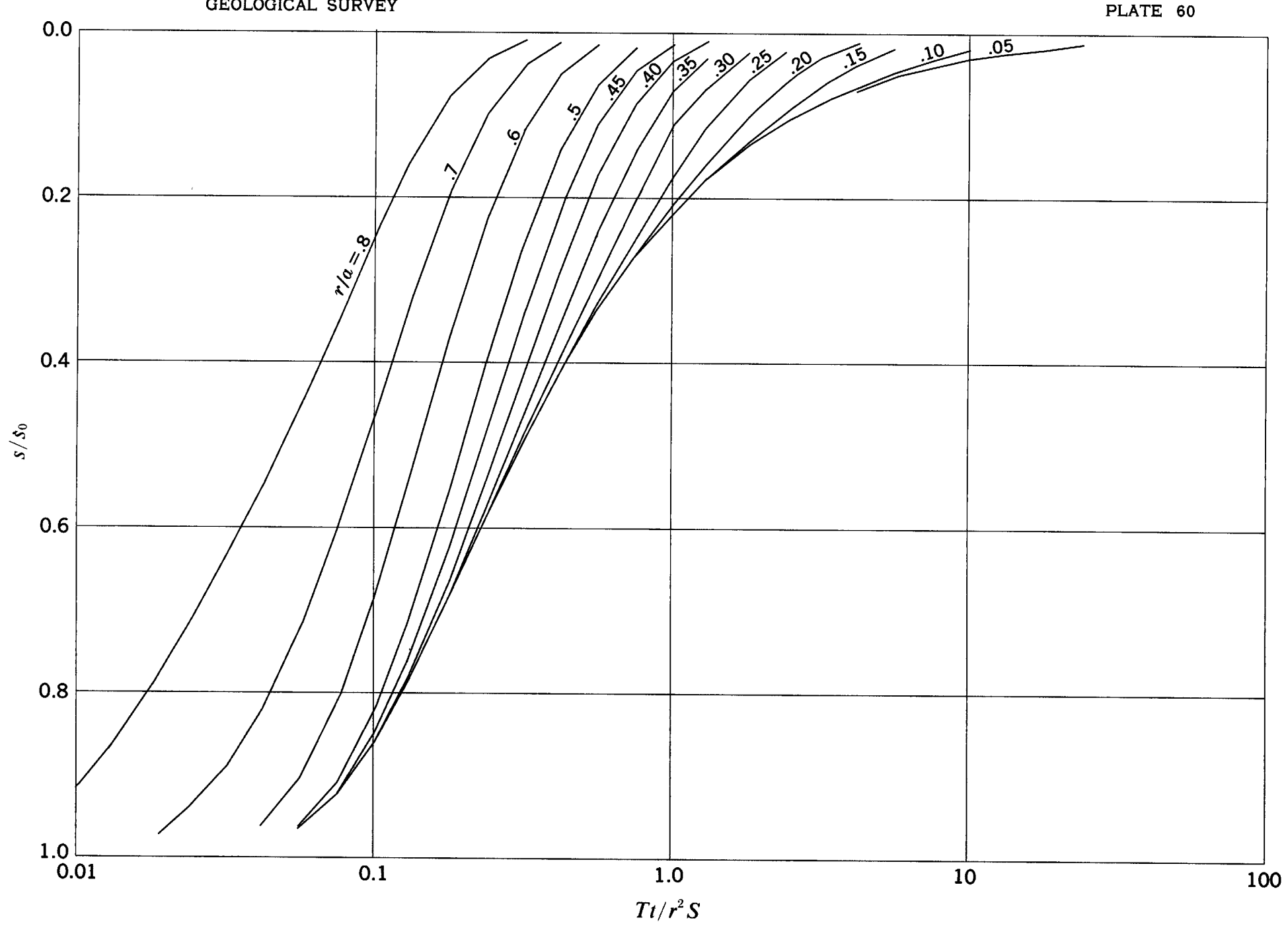

$s / s_{0}$ versus $T t / r^{2} S$ for $\theta_{0}=105^{\circ} ; \theta / \theta_{0}=0.50$ 

PLATE 61

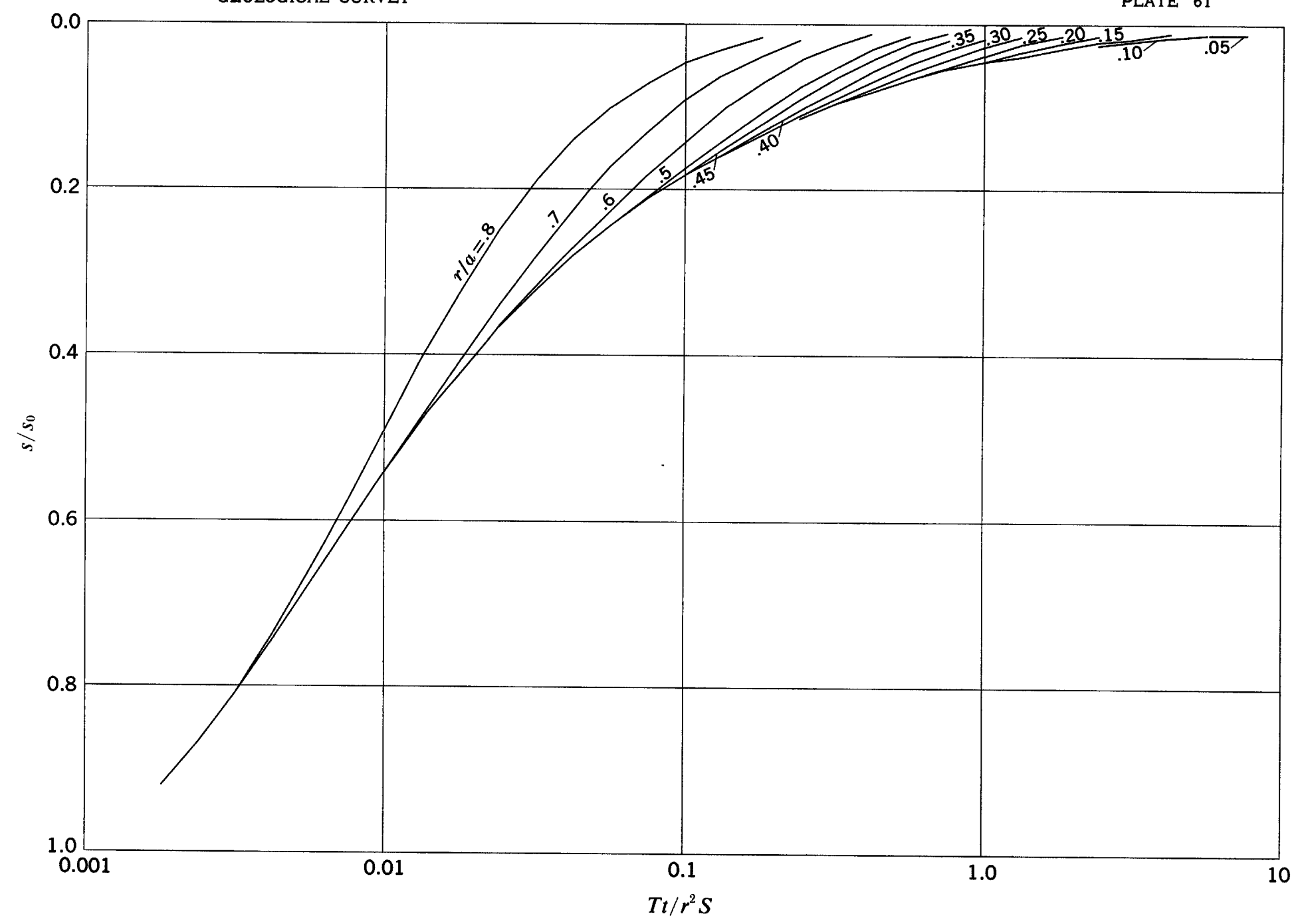

$s / s_{0}$ versus $T t / r^{2} S$ for $\theta_{0}=120^{\circ} ; \theta / \theta_{0}=0.05$ 


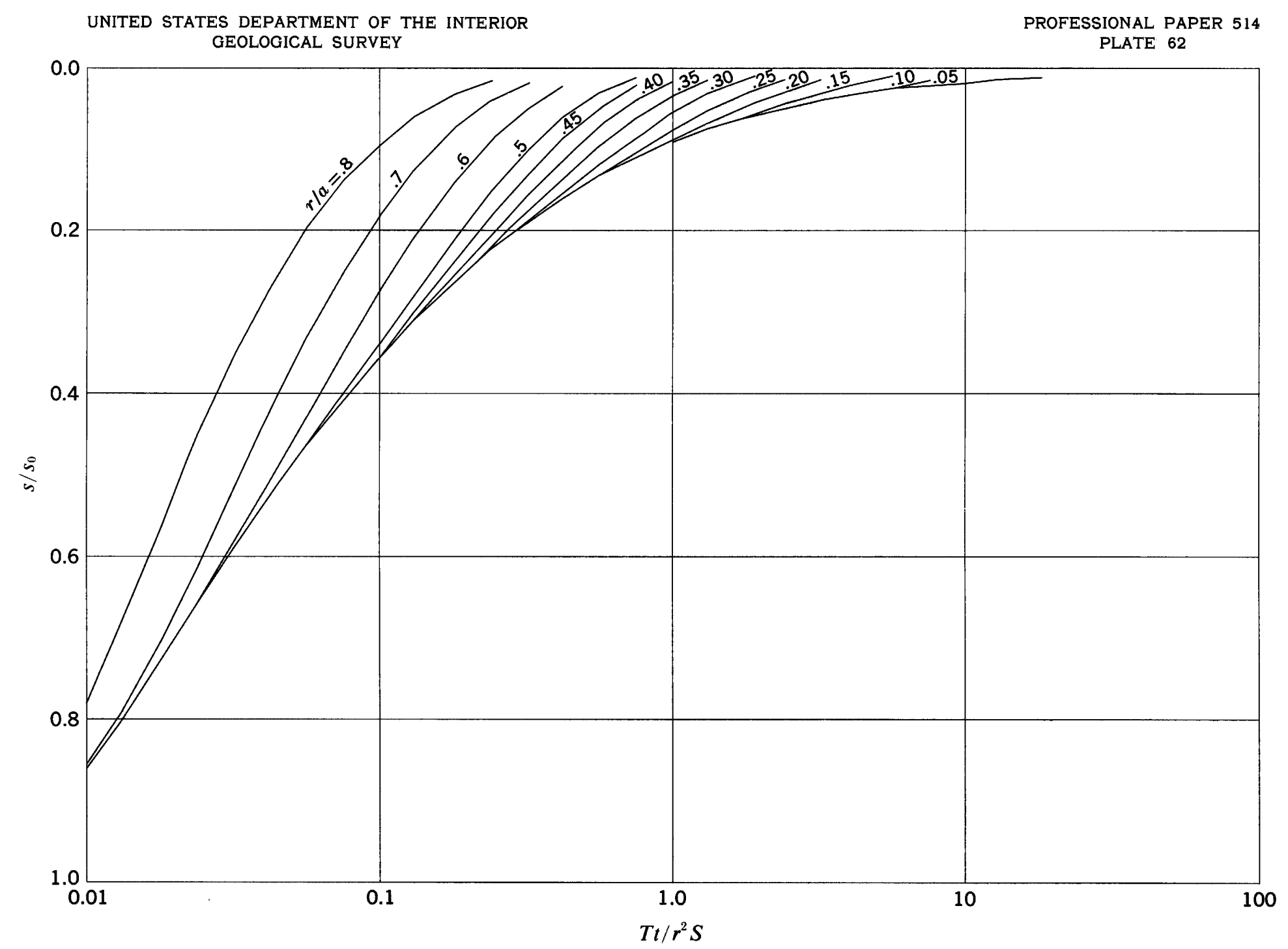

$s / s_{0}$ versus $T t / r^{2} S$ for $\theta_{0}=120^{\circ} ; \theta / \theta_{0}=0.10$ 


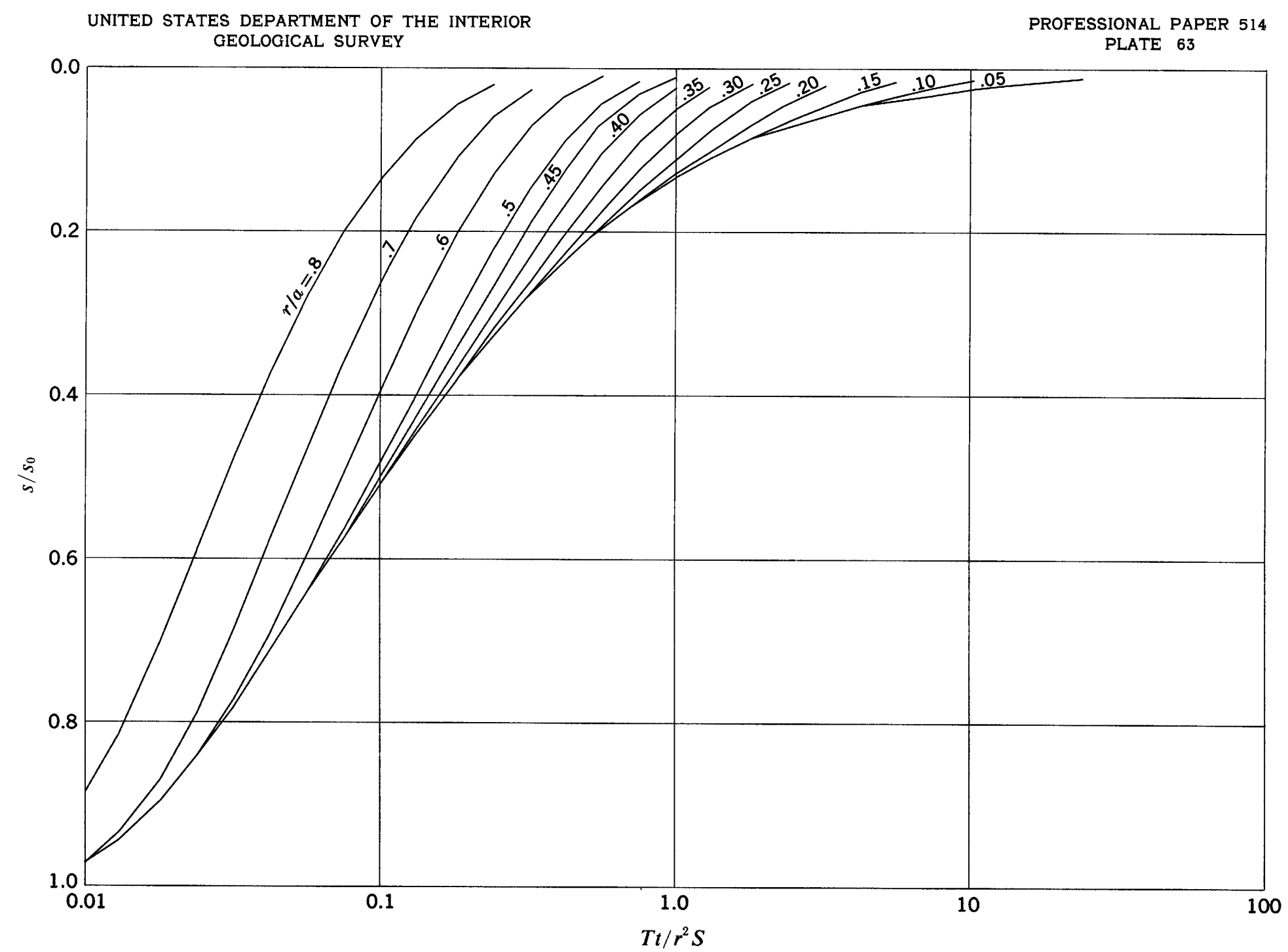

$s / s_{0}$ versus $T t / r^{2} S$ for $\theta_{0}=120^{\circ} ; \theta / \theta_{0}=0.15$ 

GEOLOGICAL SURVEY

PROFESSIONAL RAPER 514

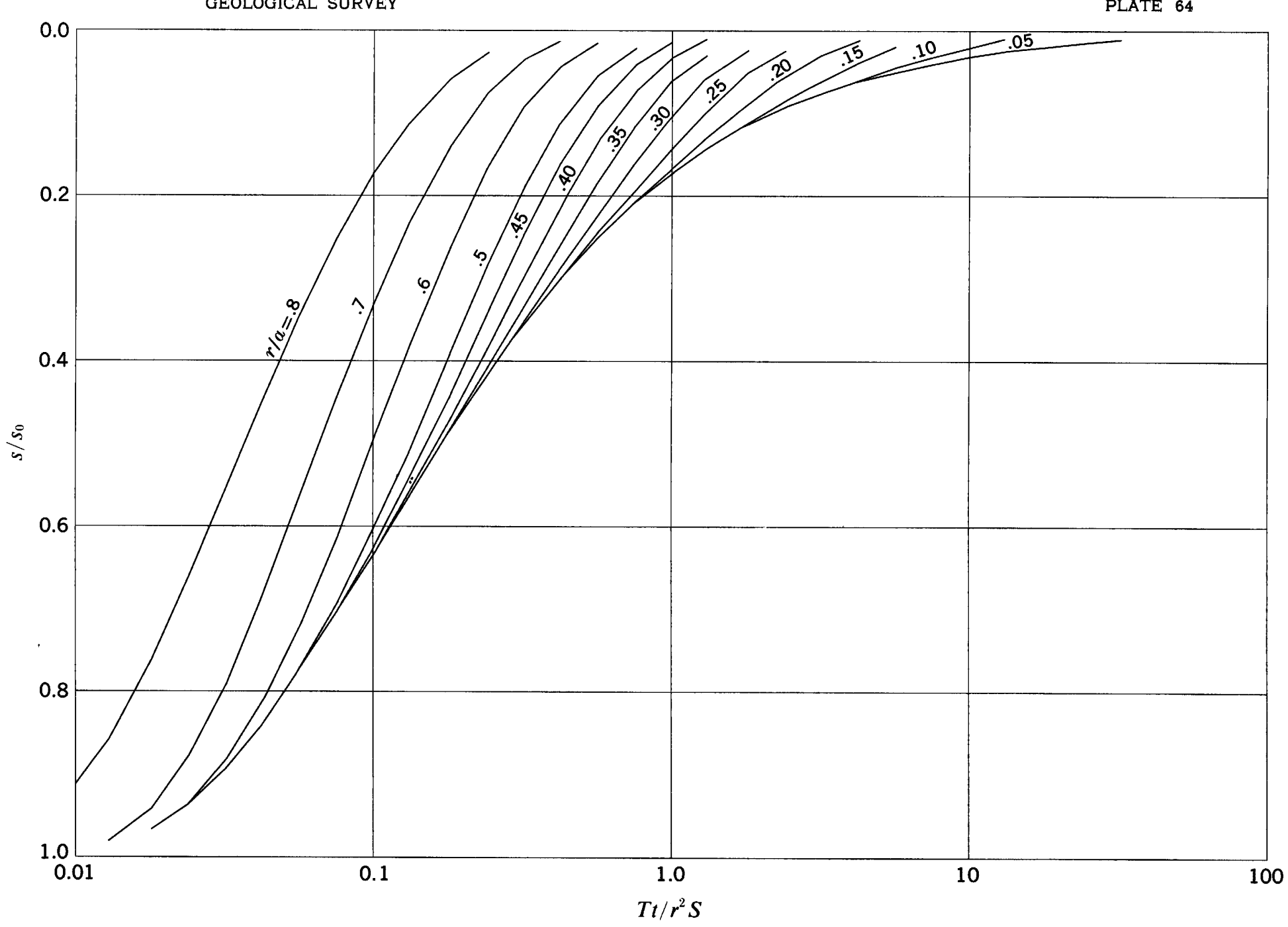

$s / s_{0}$ versus $T t / r^{2} S$ for $\theta_{0}=120^{\circ} ; \theta / \theta_{0}=0.20$ 
UNITED STATES DEPARTMENT OF THE INTERIOR GEOLOGICAL SURVEY

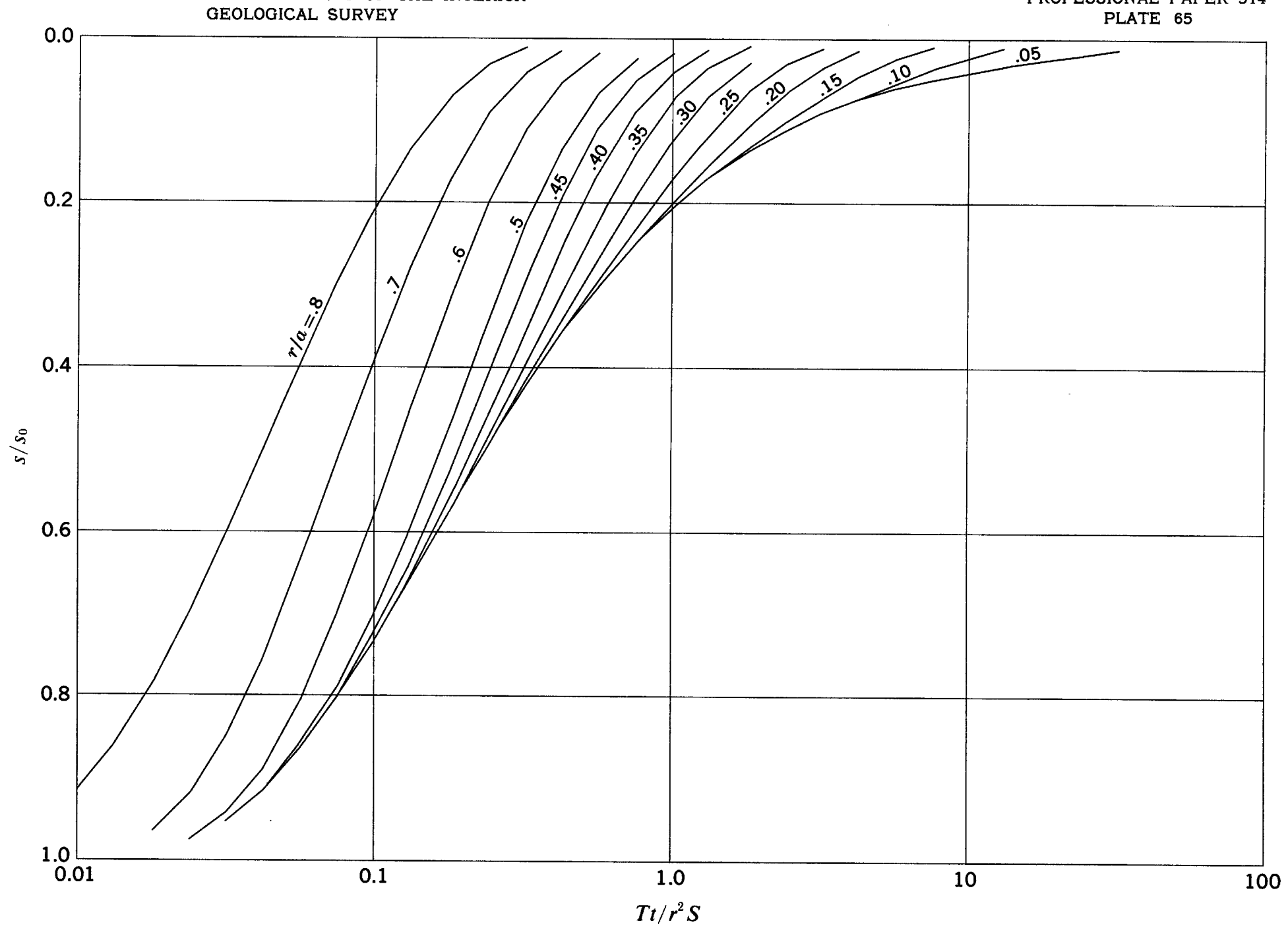

$s / s_{0}$ versus $T t / r^{2} S$ for $\theta_{0}=120^{\circ} ; \theta / \theta_{0}=0.25$ 
UNITED STATES DEPARTMENT OF THE INTERIOR GEOLOGICAL SURVEY
PROFESSIONAL PAPER 514 PLATE 66

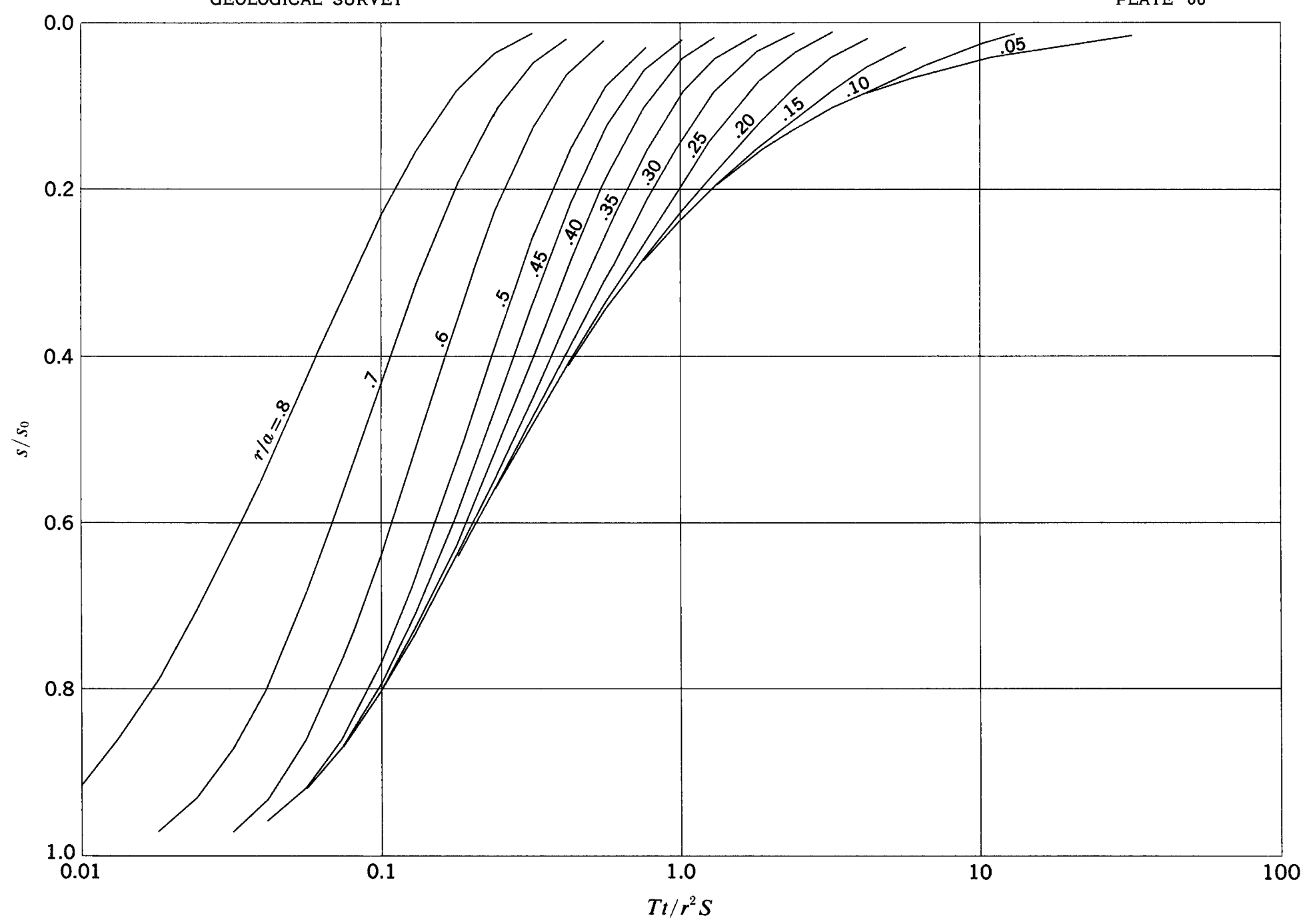

$s / s_{0}$ versus $T t / r^{2} S$ for $\theta_{0}=120^{\circ} ; \theta / \theta_{0}=0.30$ 
PLATE 67

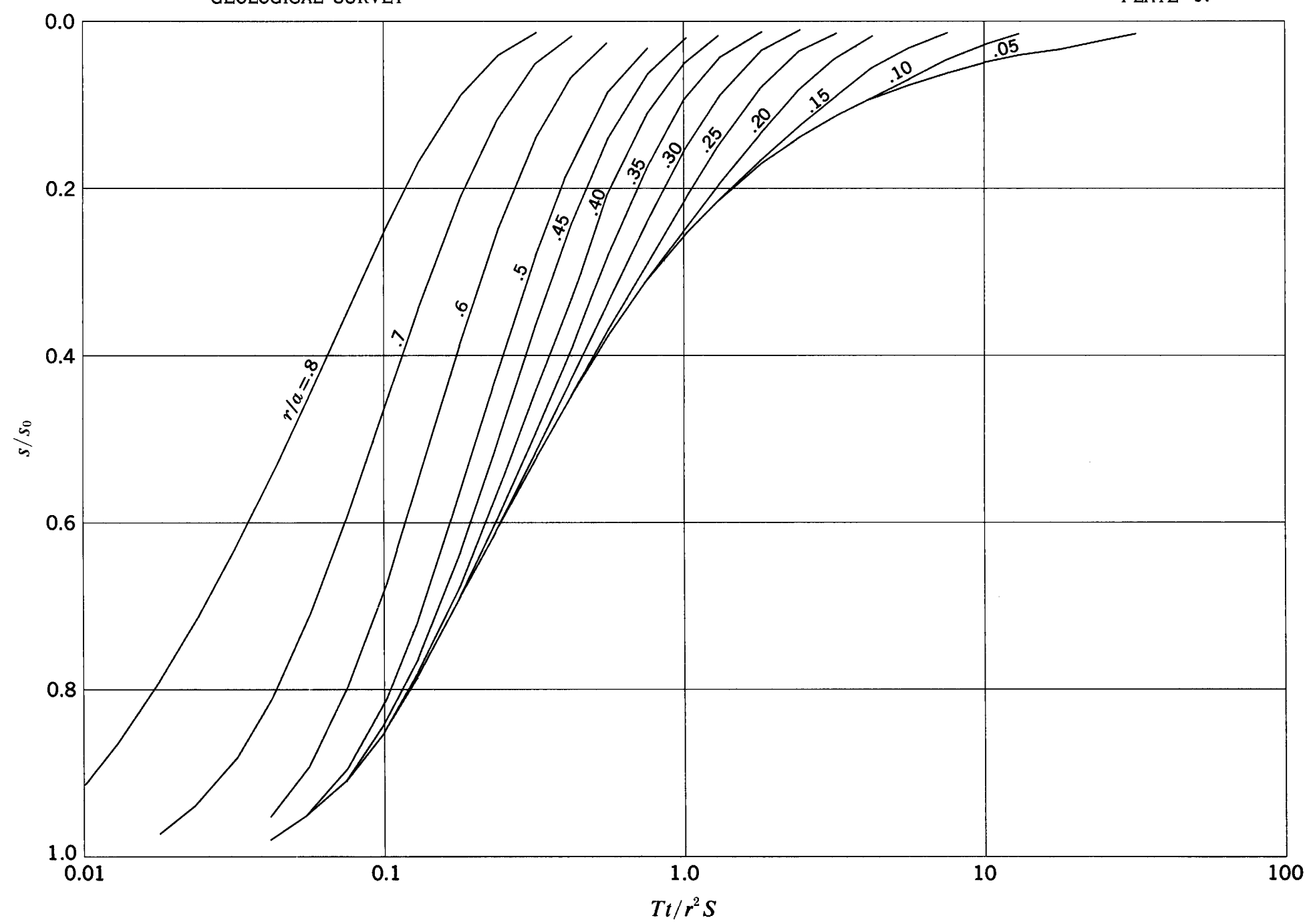

$s / s_{0}$ versus $T t / r^{2} S$ for $\theta_{0}=120^{\circ} ; \theta / \theta_{0}=0.35$ 


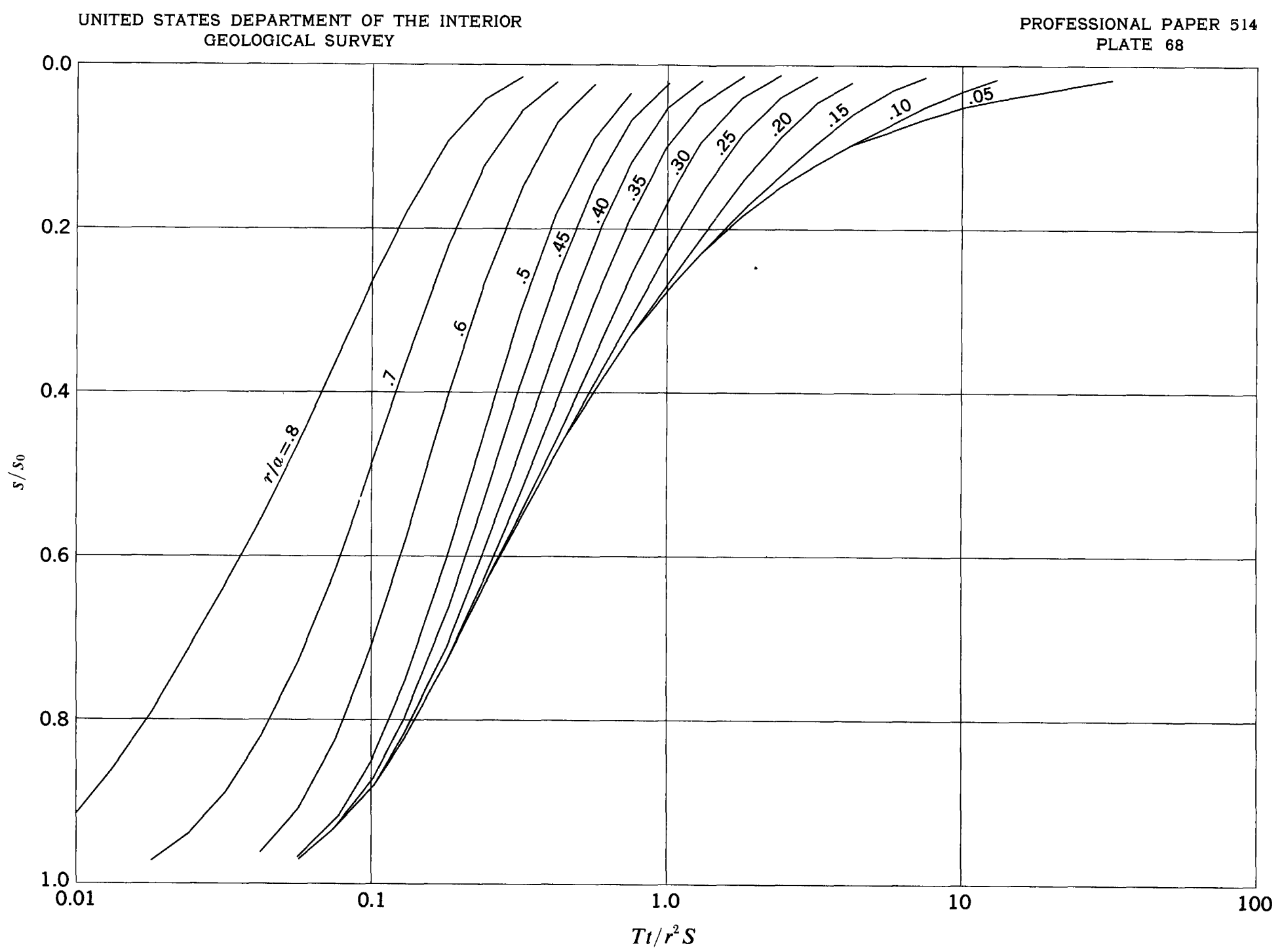

$s / s_{0}$ versus $T t / r^{2} S$ for $\theta_{0}=120^{\circ} ; \theta / \theta_{0}=0.40$ 


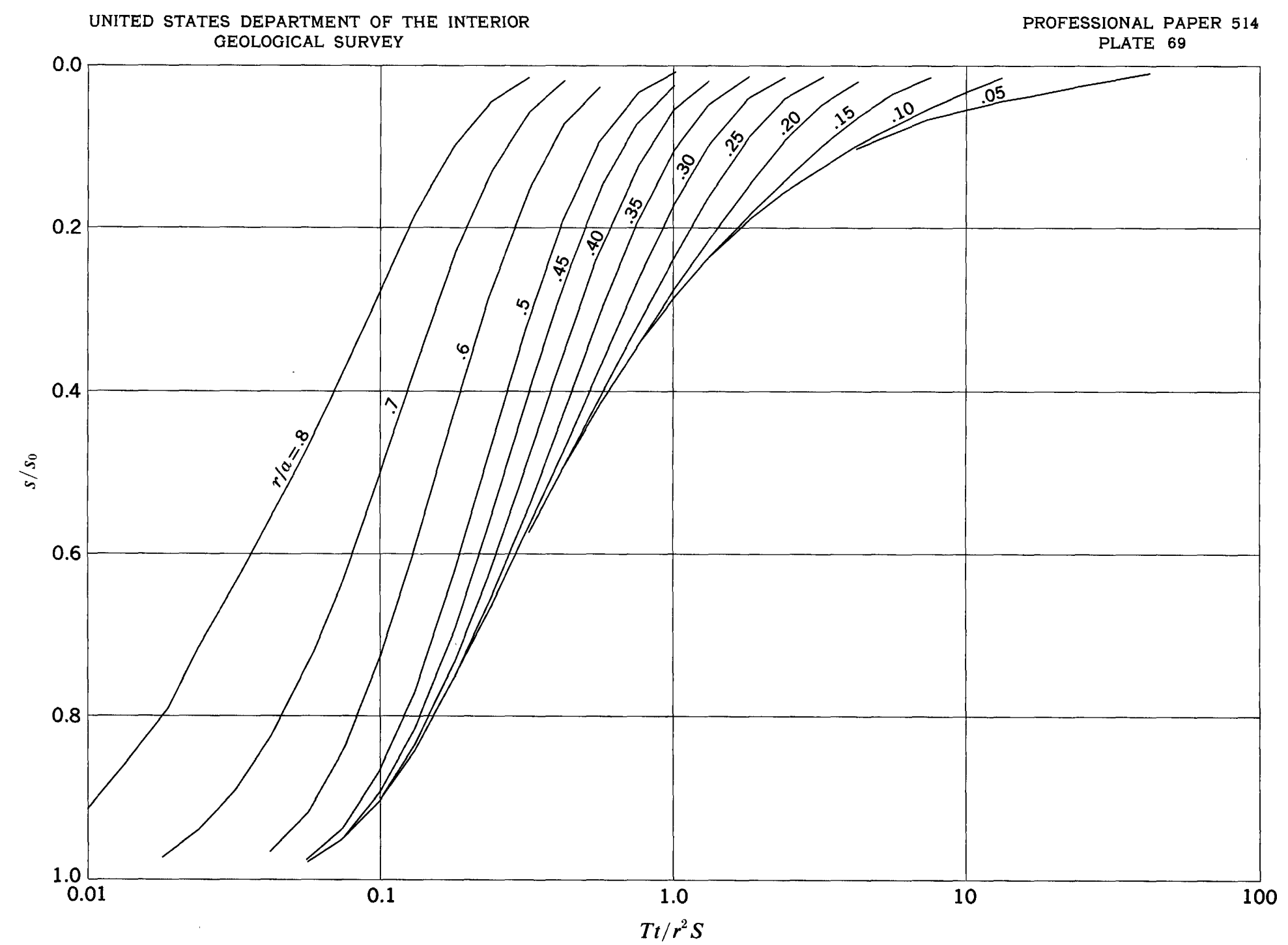

$s / s_{0}$ versus $T t / r^{2} S$ for $\theta_{0}=120^{\circ} ; \theta / \theta_{0}=0.45$ 
UNITED STATES DEPARTMENT OF THE INTERIOR

PROFESSIONAL PAPER 514 GEOLOGICAL SURVEY PLATE 70

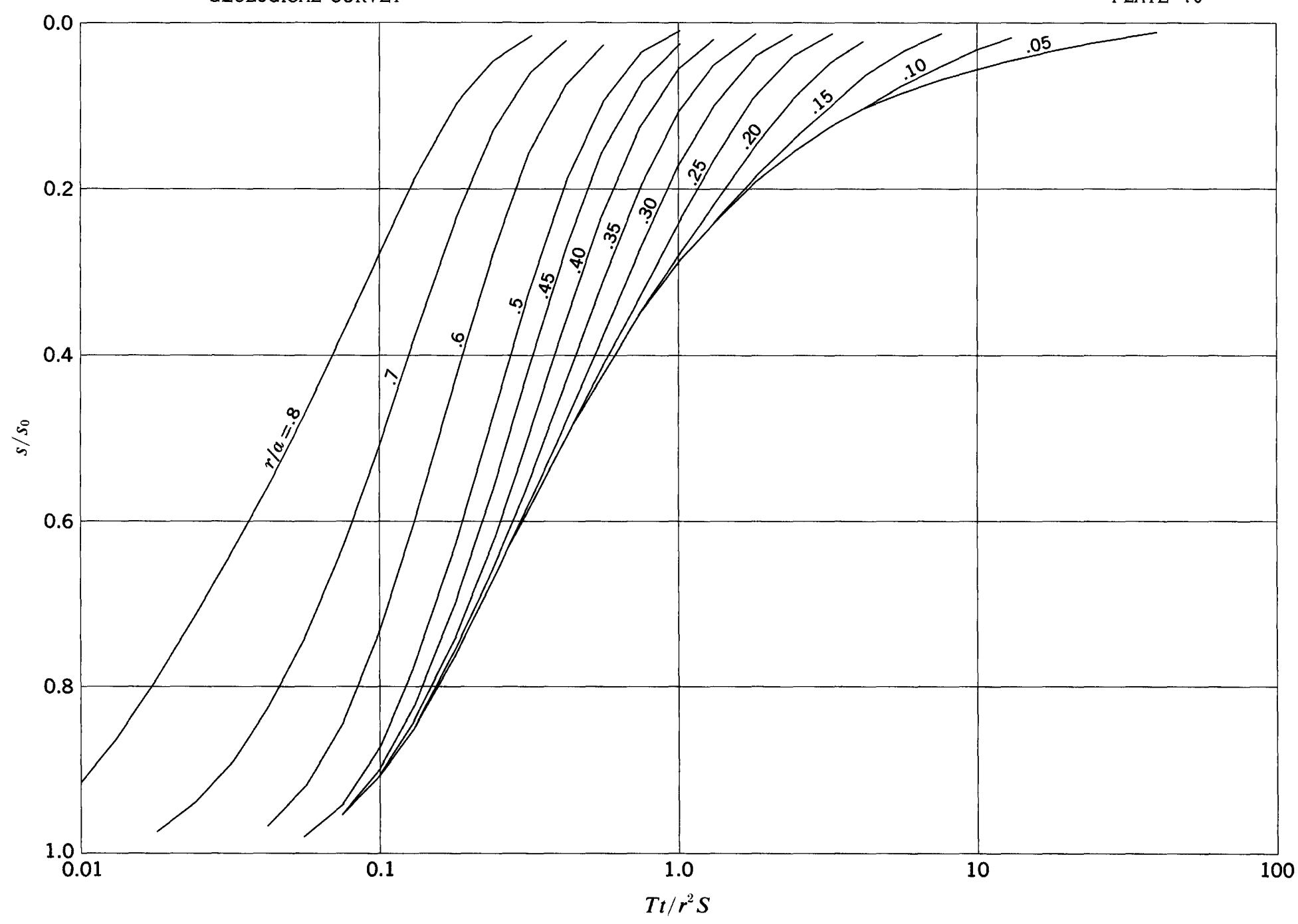

$s / s_{0}$ versus $T t / r^{2} S$ for $\theta_{0}=120^{\circ} ; \theta / \theta_{0}=0.50$ 

GEOLOGICAL SURVEY

PLATE 71

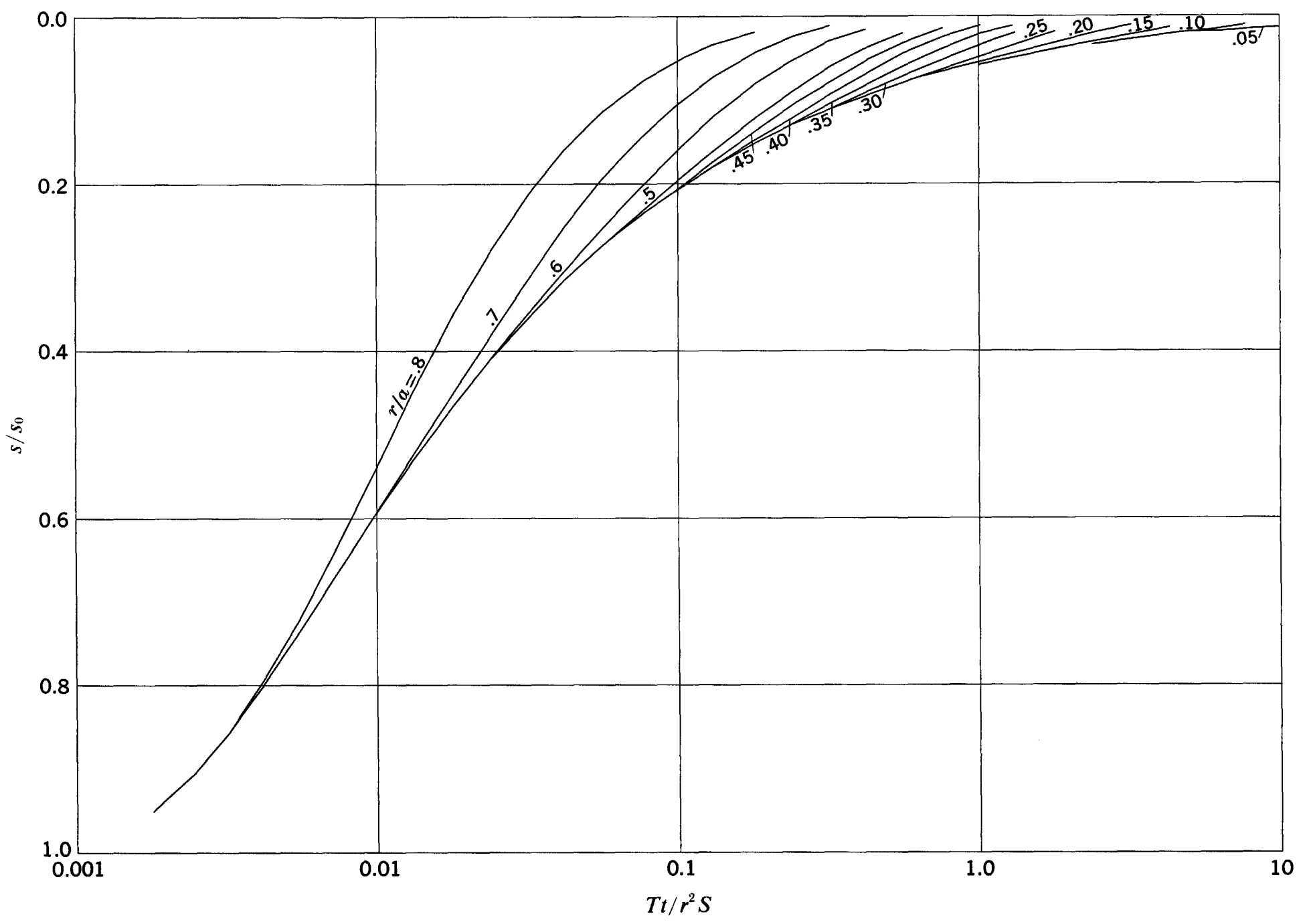

$s / s_{0}$ versus $T t / r^{2} S$ for $\theta_{0}=135^{\circ} ; \theta / \theta_{0}=0.05$ 
UNITED STATES DEPARTMENT OF THE INTERIOR GEOLOGICAL SURVEY

PROFESSIONAL PAPER 514 PLATE 72

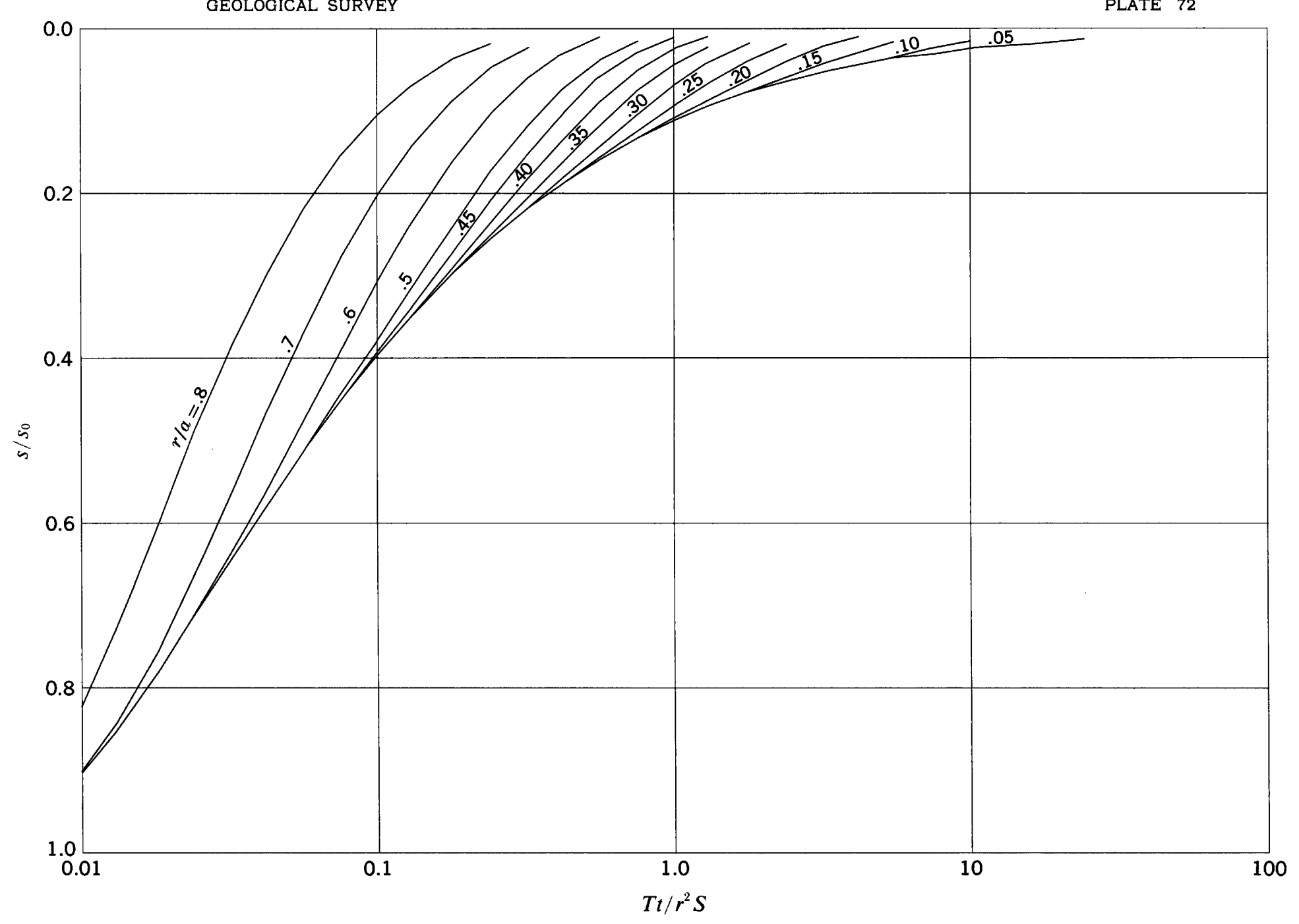

$s / s_{0}$ versus $T t / r^{2} S$ for $\theta_{0}=135^{\circ} ; \theta / \theta_{0}=0.10$ 

GEOLOGICAL SURVEY PLATE 73

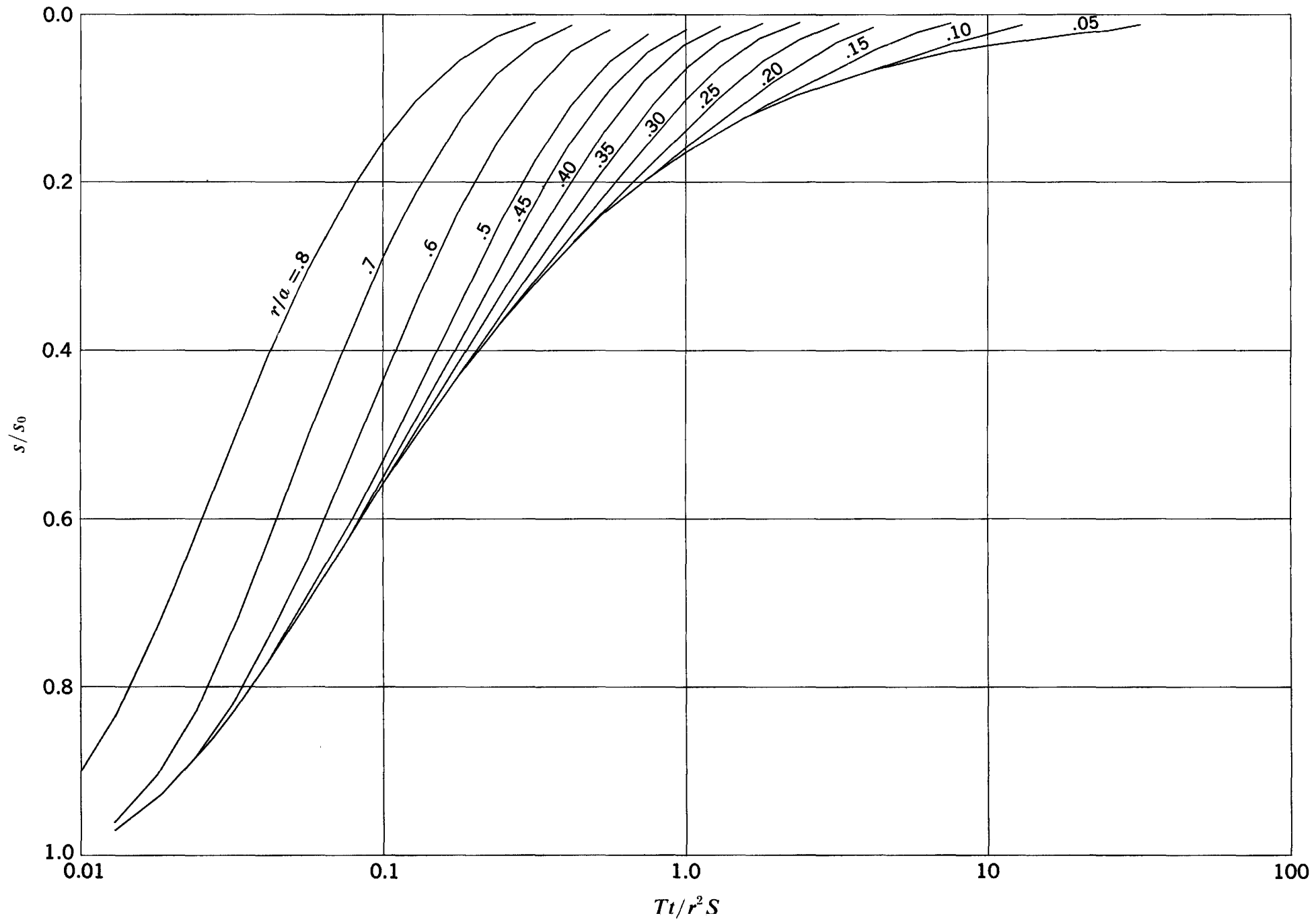

$s / s_{0}$ versus $T t / r^{2} S$ for $\theta_{0}=135^{\circ} ; \theta / \theta_{0}=0.15$ 
UNITED STATES DEPARTMENT OF THE INTERIOR GEOLOGICAL SURVEY
PROFESSIONAL PAPER 514 PLATE 74

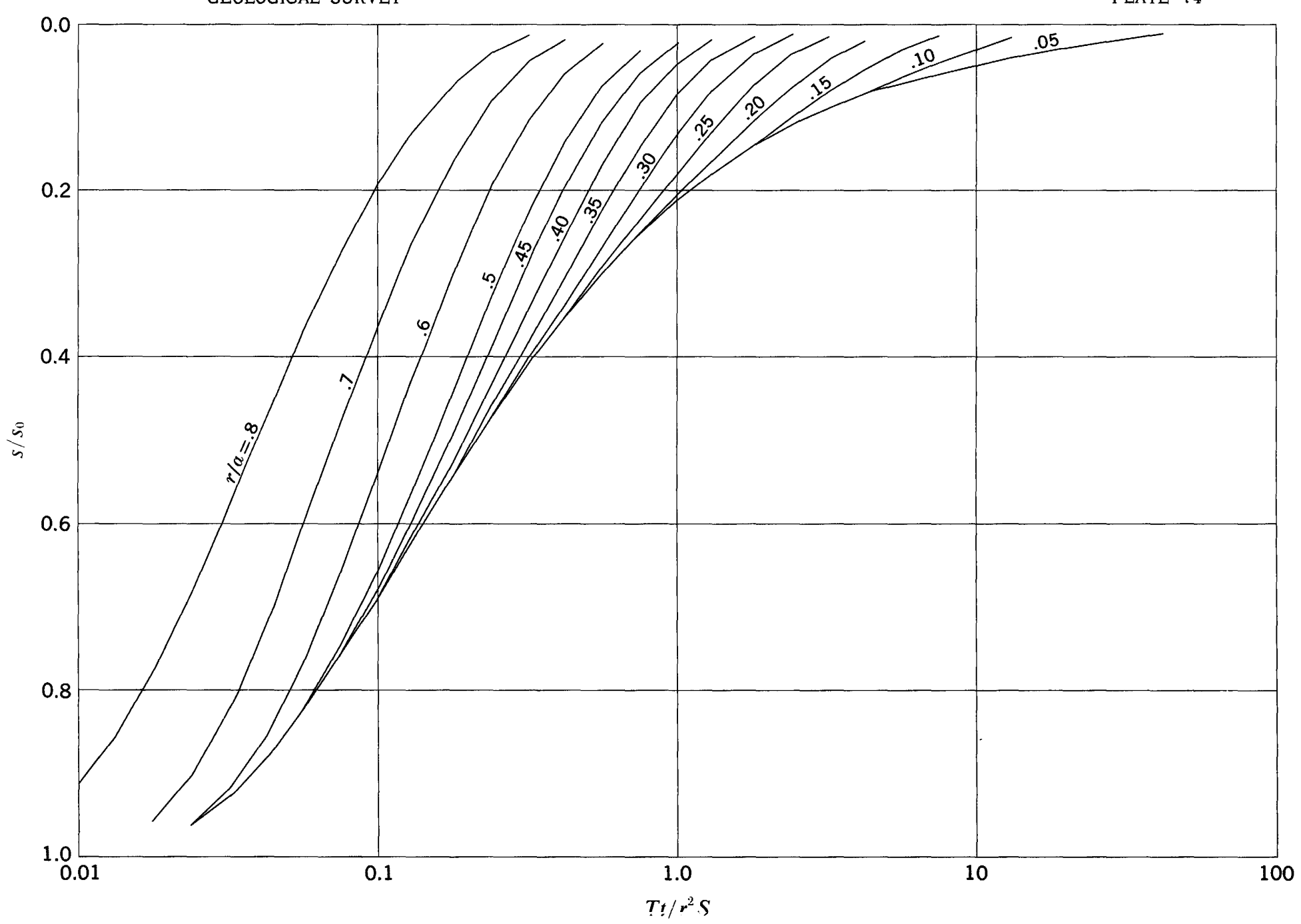

$s / s_{0}$ versus $T t / r^{2} S$ for $\theta_{0}=135^{\circ} ; \theta / \theta_{0}=0.20$ 

GEOLOGICAL SURVEY PLATE 75

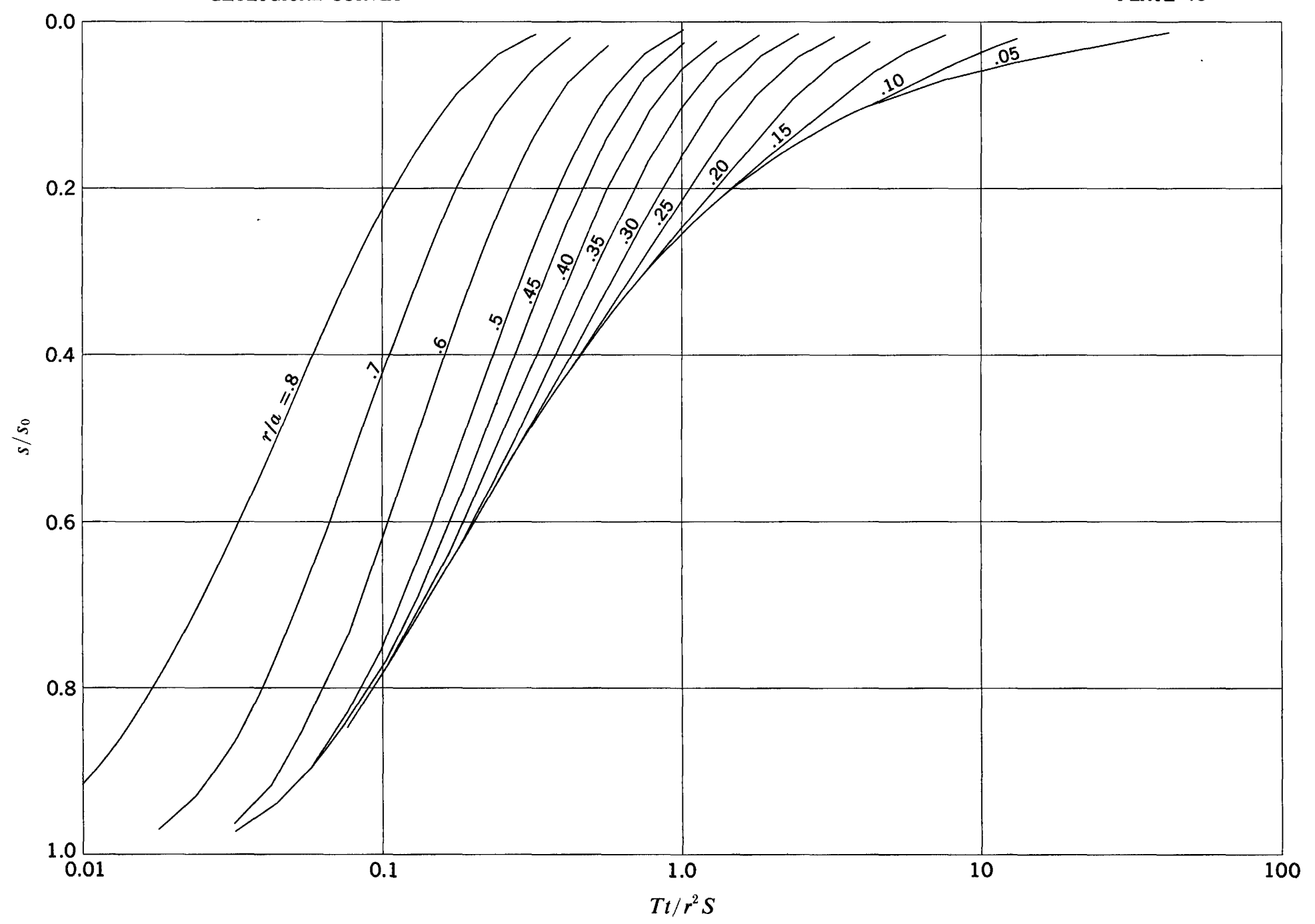

$s / s_{0}$ versus $T t / r^{2} S$ for $\theta_{0}=135^{\circ} ; \theta / \theta_{0}=0.25$ 

GEOLOGICAL SURVEY

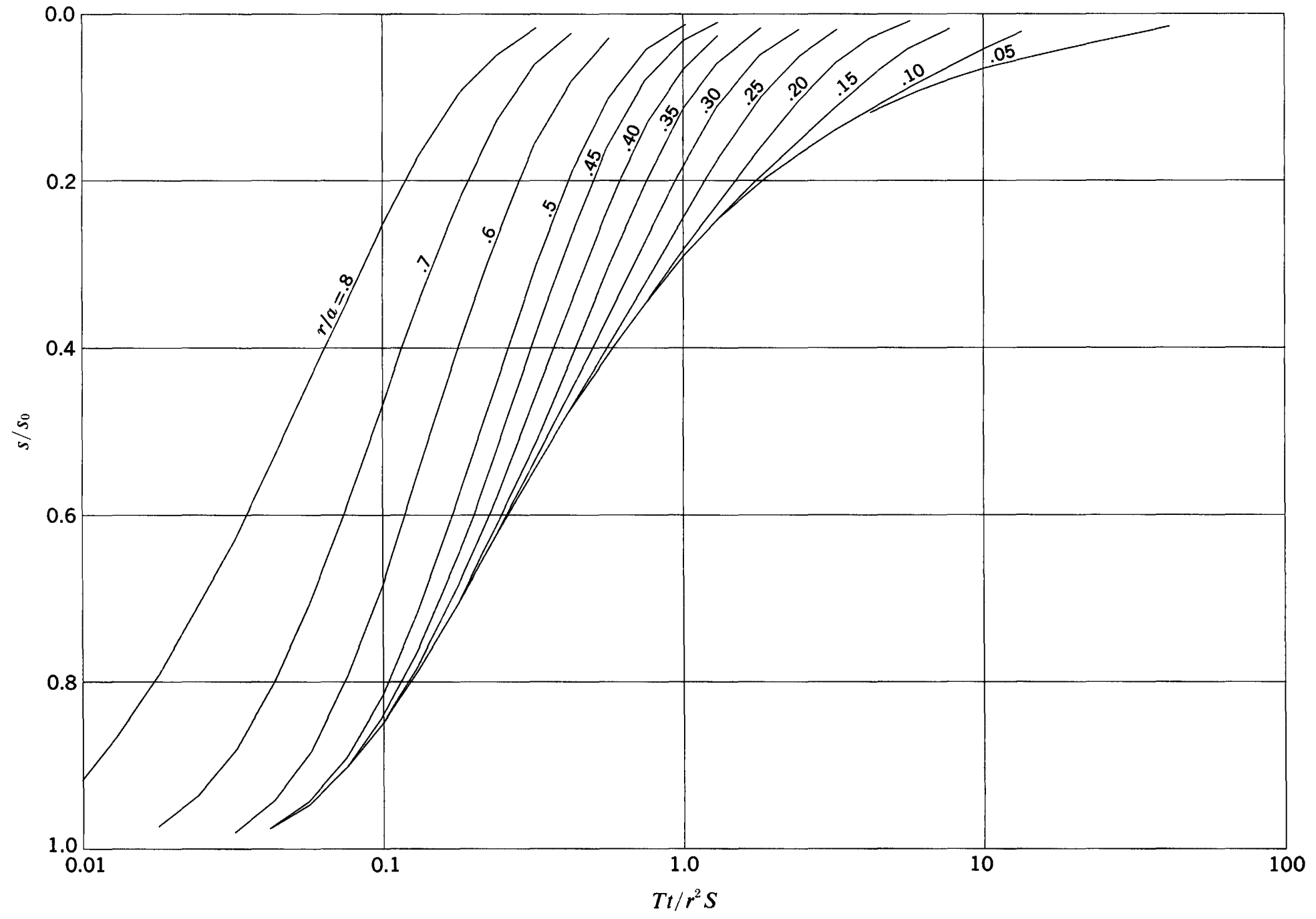

$s / s_{0}$ versus $T t / r^{2} S$ for $\theta_{0}=135^{\circ} ; \theta / \theta_{0}=0.30$ 

GEOLOGICAL SURVEY PLATE 77

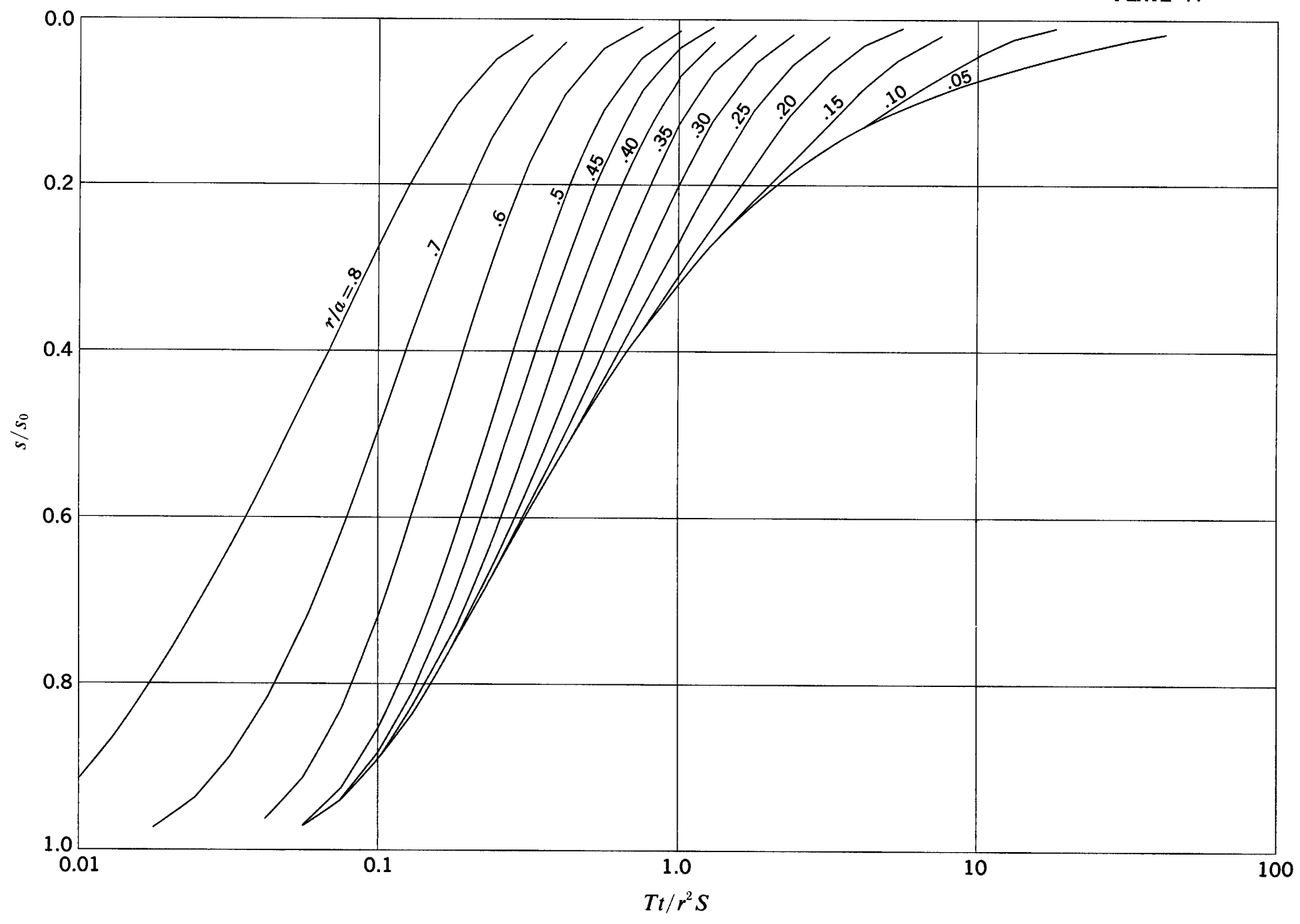

$s / s_{0}$ versus $T t / r^{2} S$ for $\theta_{0}=135^{\circ} ; \theta / \theta_{0}=0.35$ 


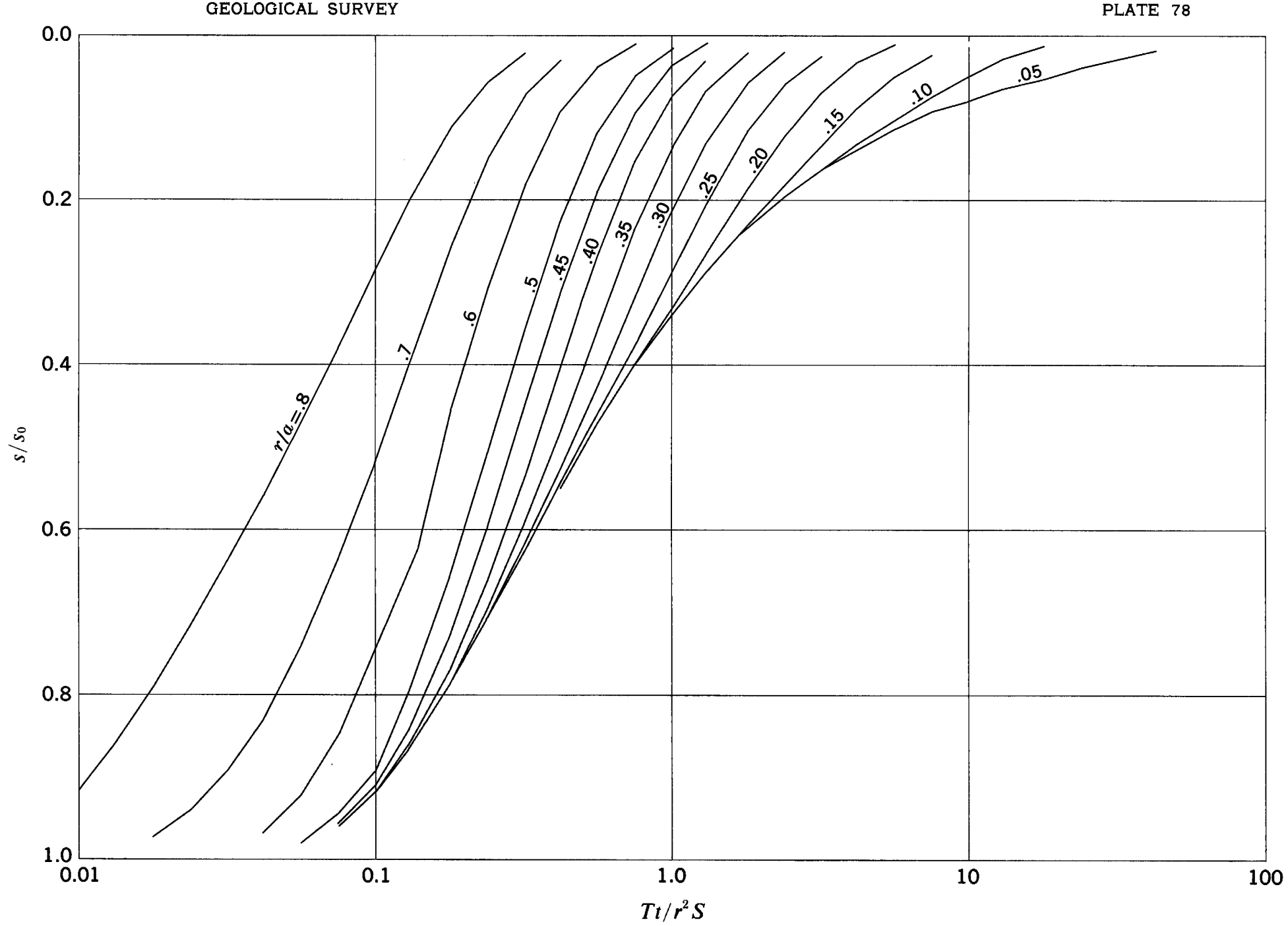

$s / s_{0}$ versus $T t / r^{2} S$ for $\theta_{0}=135^{\circ} ; \theta / \theta_{0}=0.40$ 
UNITED STATES DEPARTMENT OF THE INTERIOR GEOLOGICAL SURVEY

PROFESSIONAL PAPER 514 PLATE 79

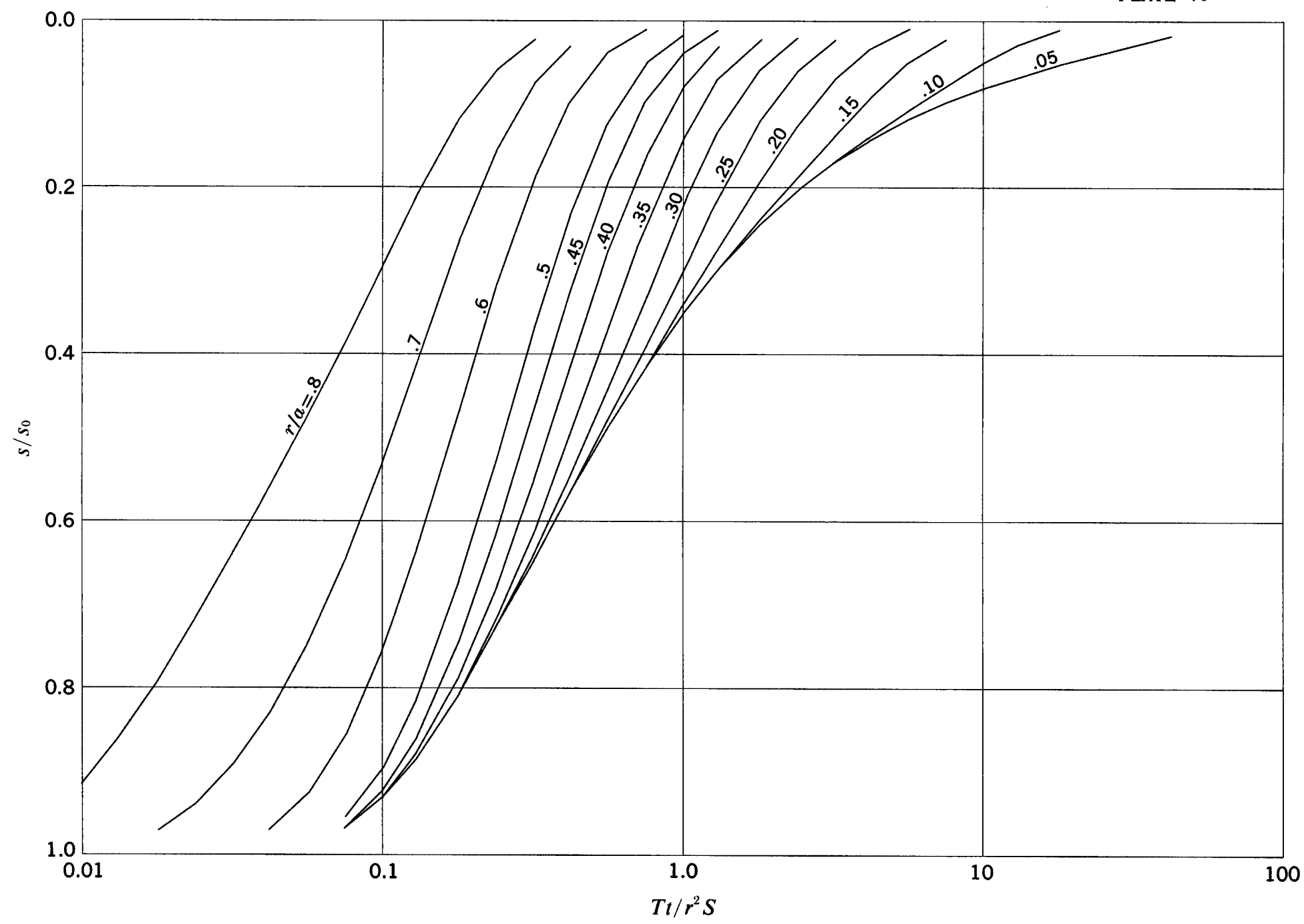

$s / s_{0}$ versus $T t / r^{2} S$ for $\theta_{0}=135^{\circ} ; \theta / \theta_{0}=0.45$ 

PLATE 80

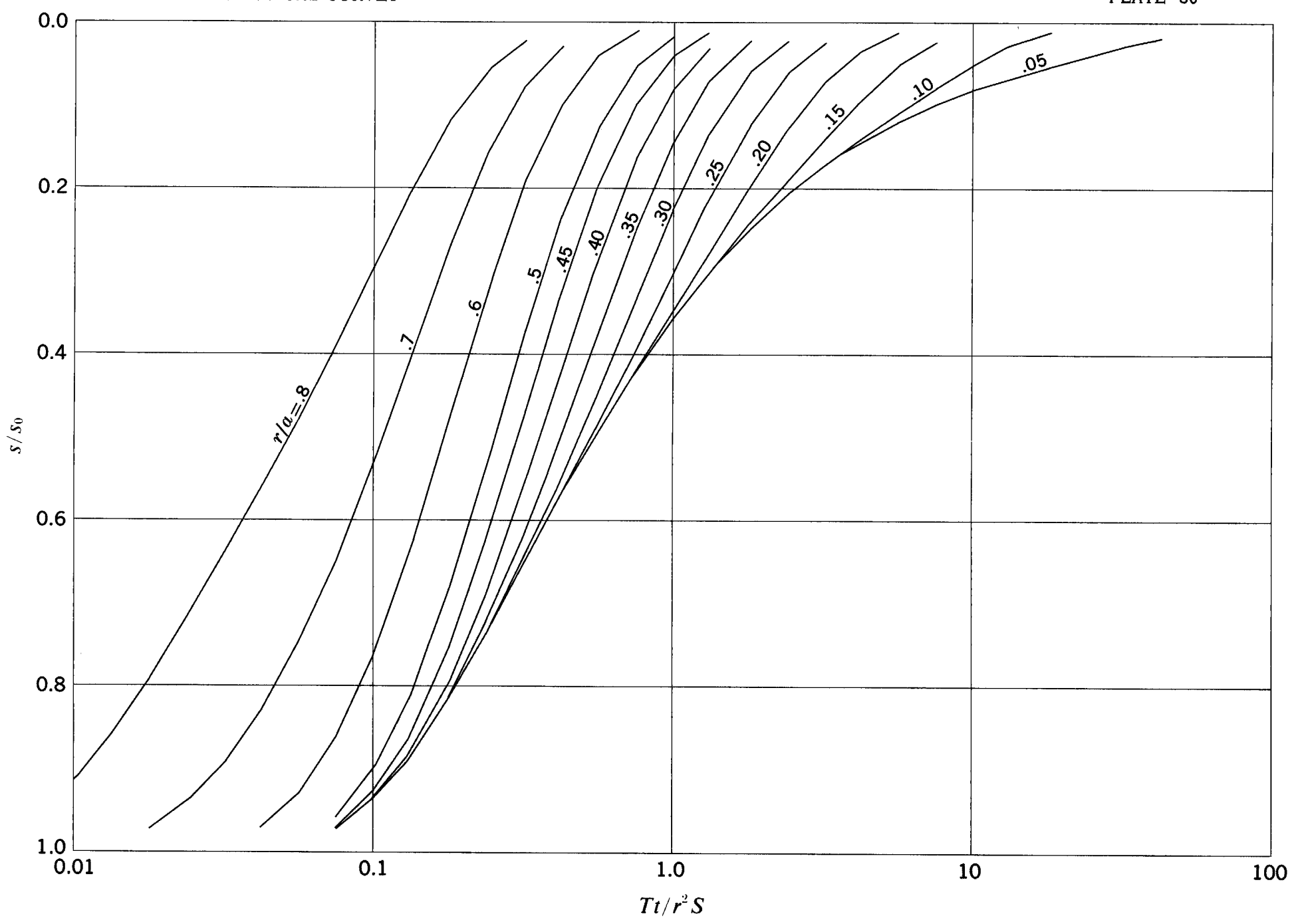

$s / s_{0}$ versus $T t / r^{2} S$ for $\theta_{0}=135^{\circ} ; \theta / \theta_{0}=0.50$ 

GEOLOGICAL SURVEY PLATE 81

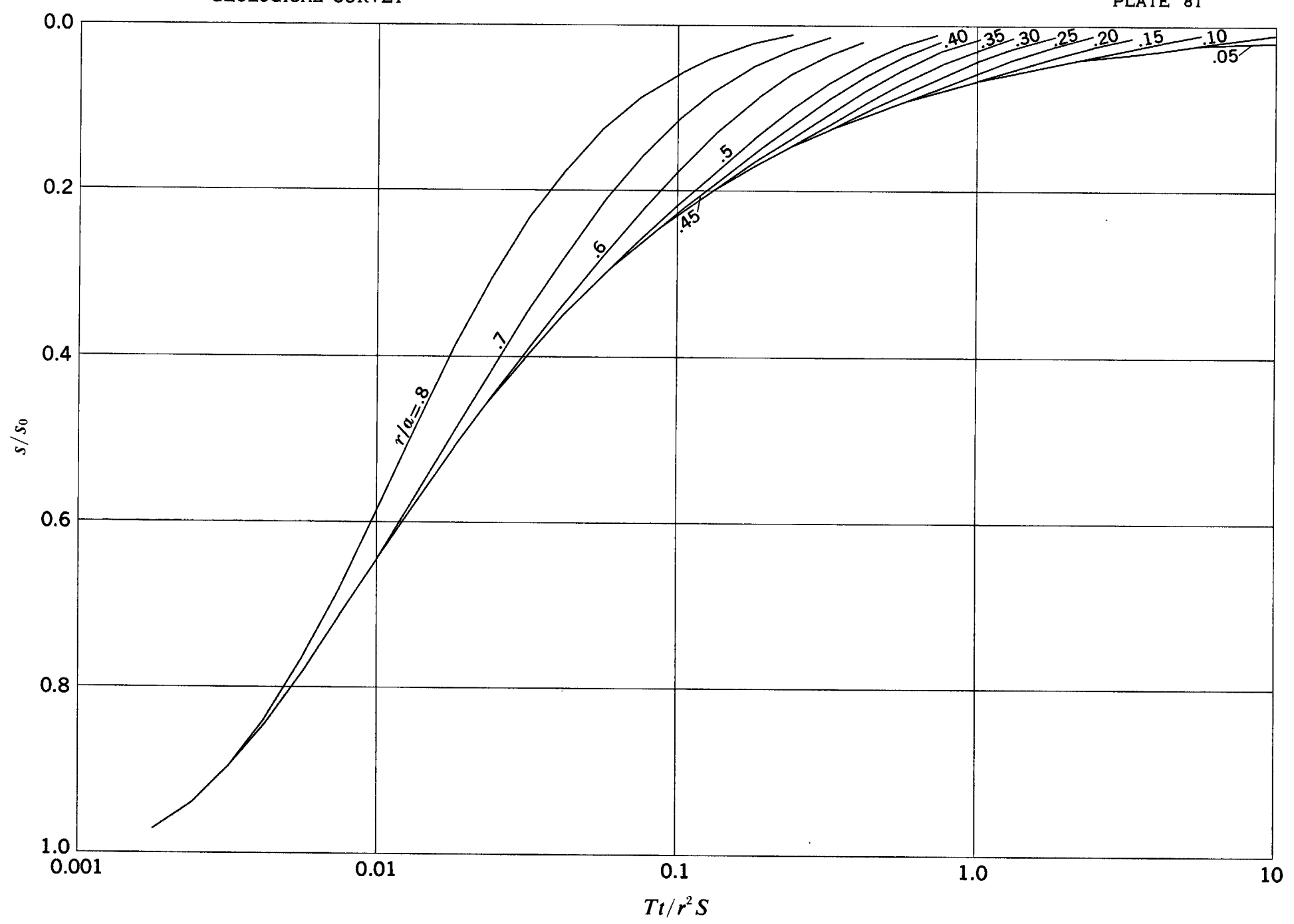

$s / s_{0}$ versus $T t / r^{2} S$ for $\theta_{0}=150^{\circ} ; \theta / \theta_{0}=0.05$ 

GEOLOGICAL SURVEY

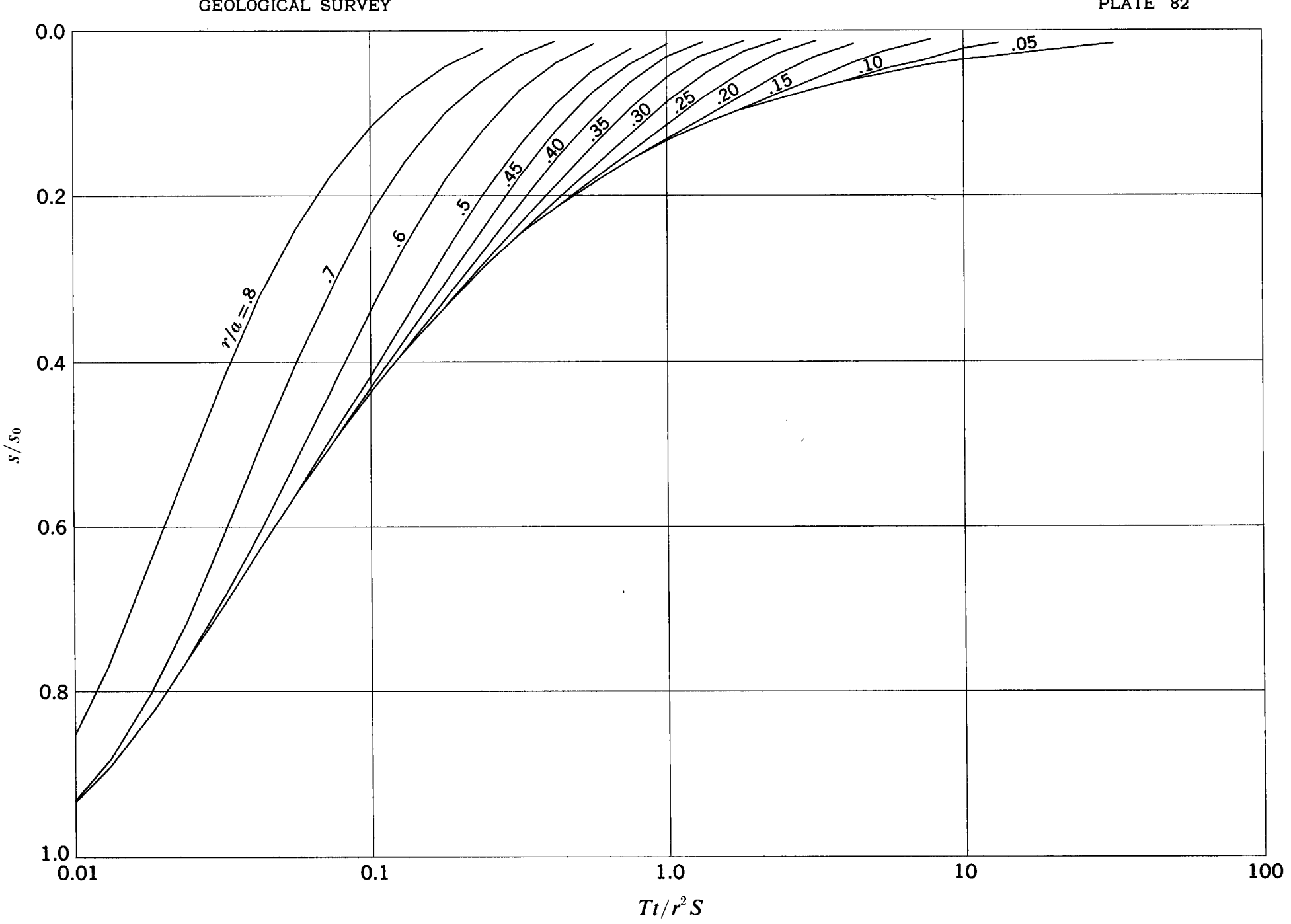

$s / s_{0}$ versus $T t / r^{2} S$ for $\theta_{0}=150^{\circ} ; \theta / \theta_{0}=0.10$ 

GEOLOGICAL SURVEY PLATE 83

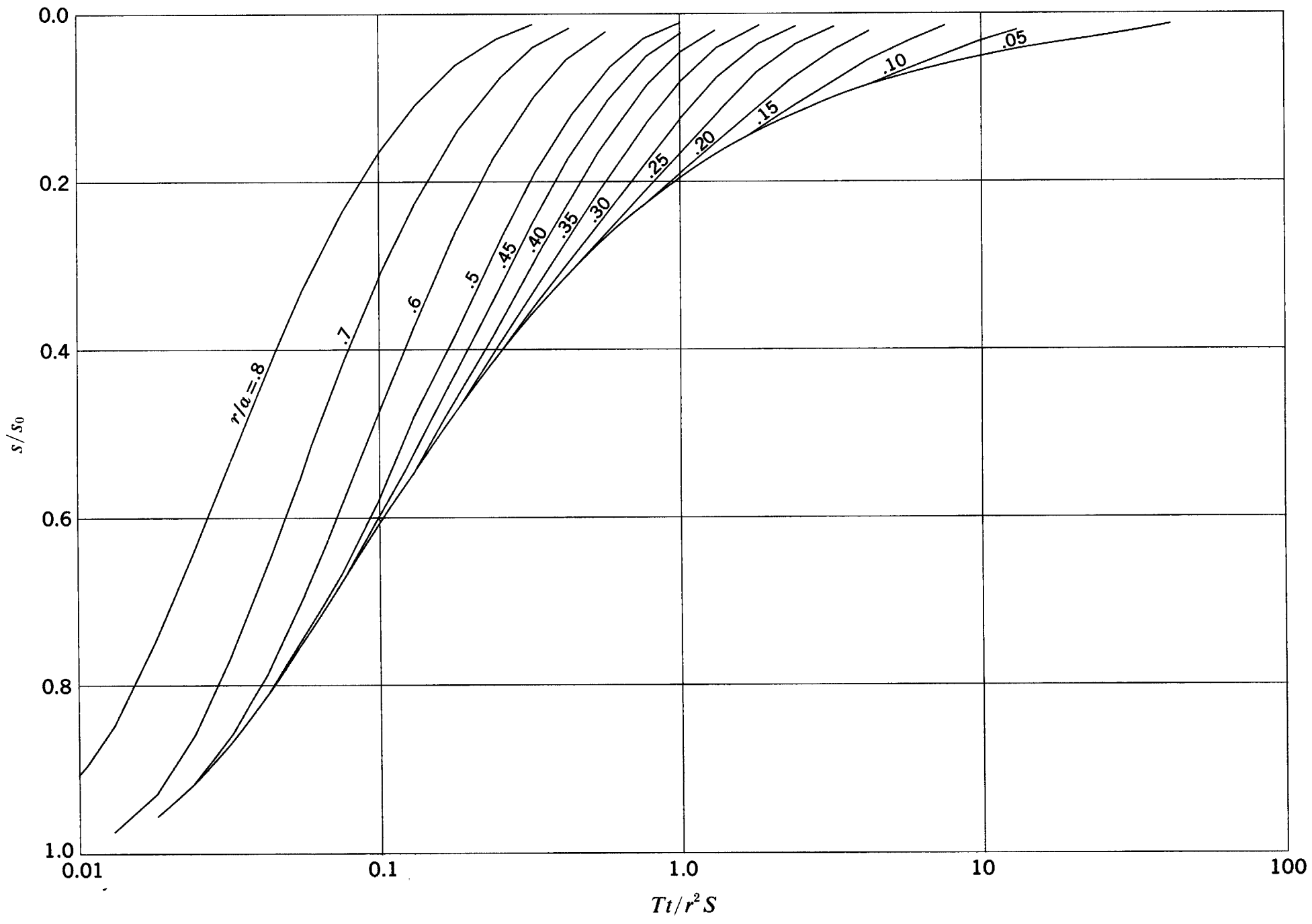

$s / s_{0}$ versus $T t / r^{2} S$ for $\theta_{0}=150^{\circ} ; \theta / \theta_{0}=0.15$ 


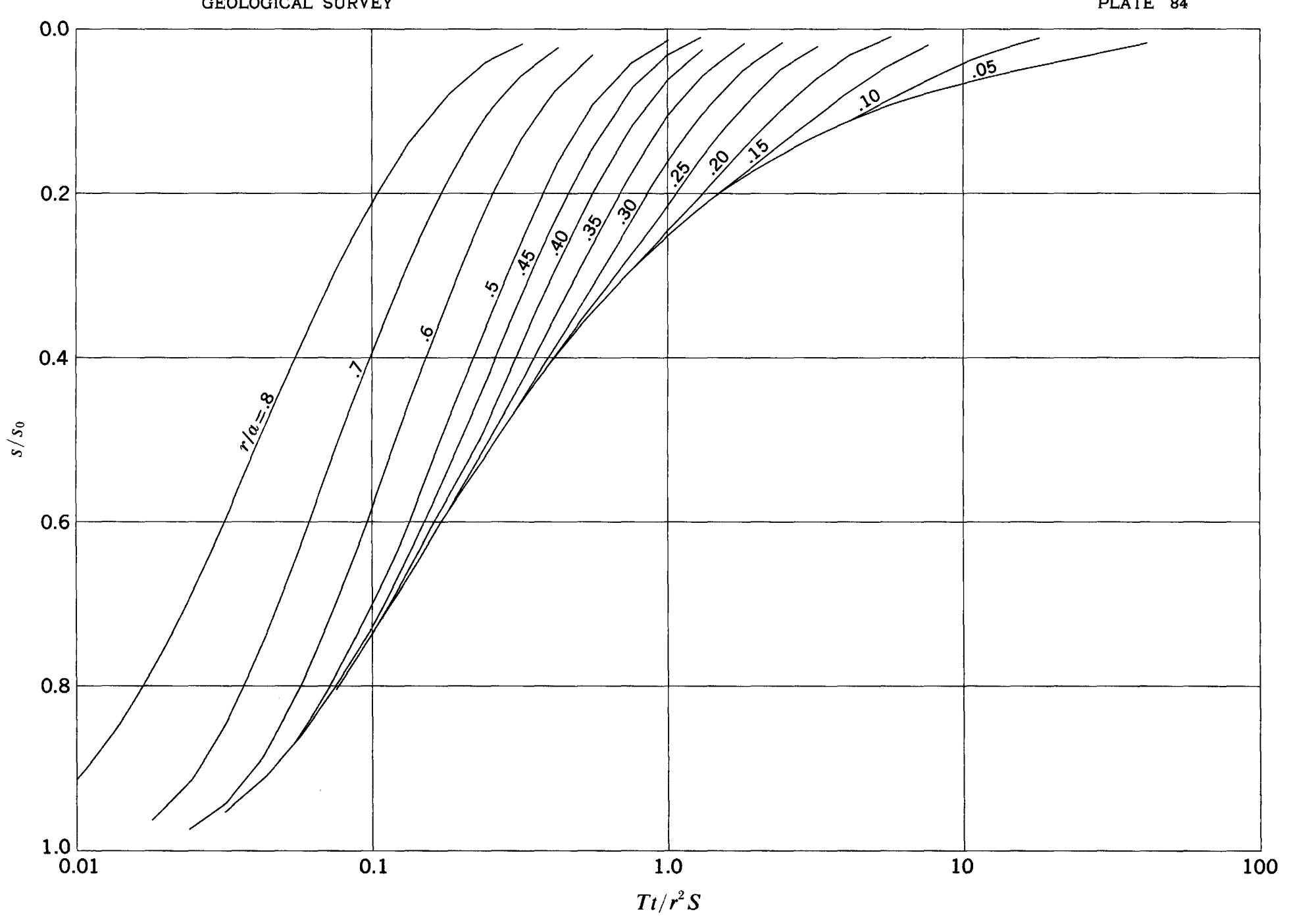

$s / s_{0}$ versus $T t / r^{2} S$ for $\theta_{0}=150^{\circ} ; \theta / \theta_{0}=0.20$ 


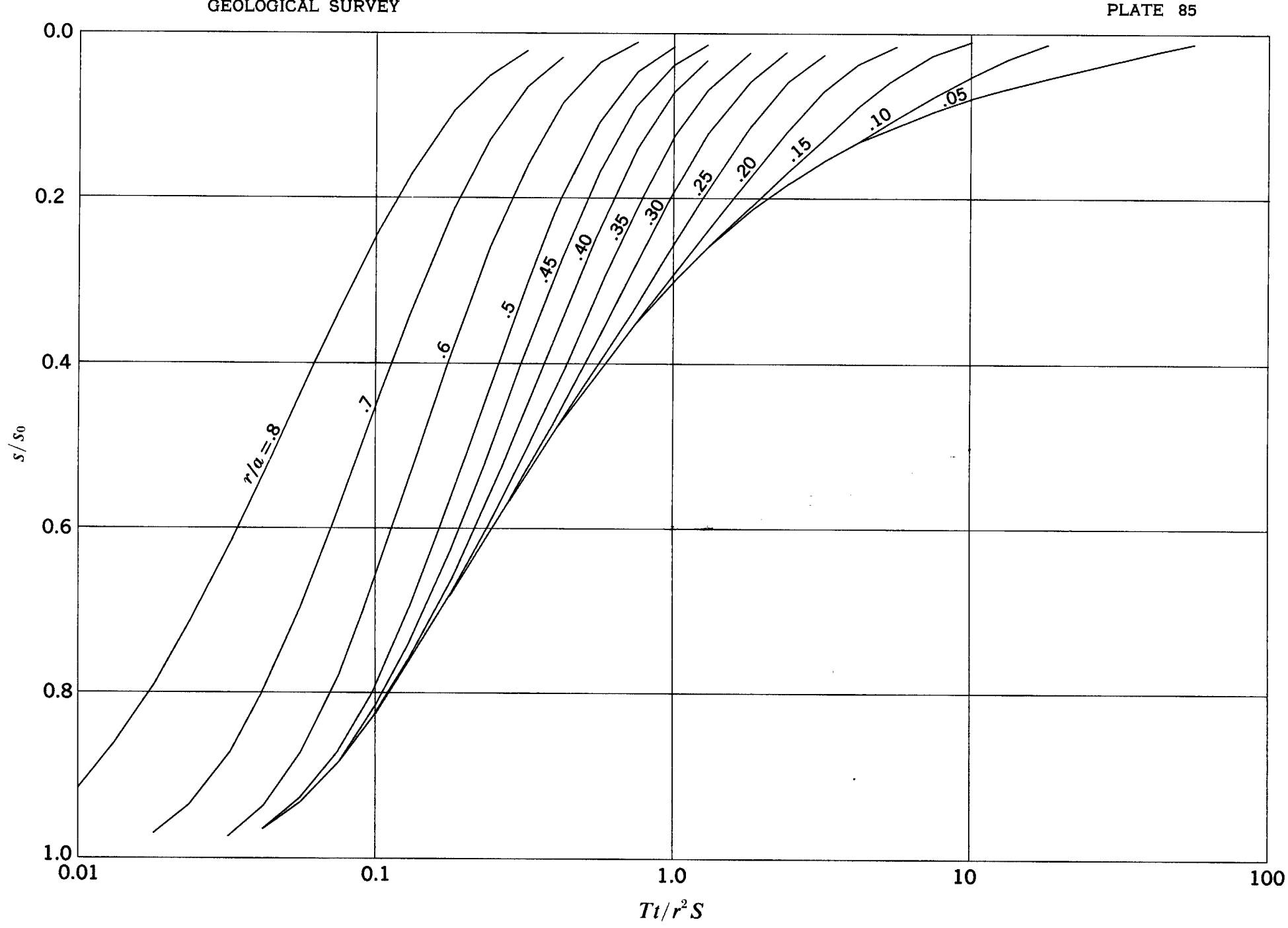

$s / s_{0}$ versus $T t / r^{2} S$ for $\theta_{0}=150^{\circ} ; \theta / \theta_{0}=0.25$ 


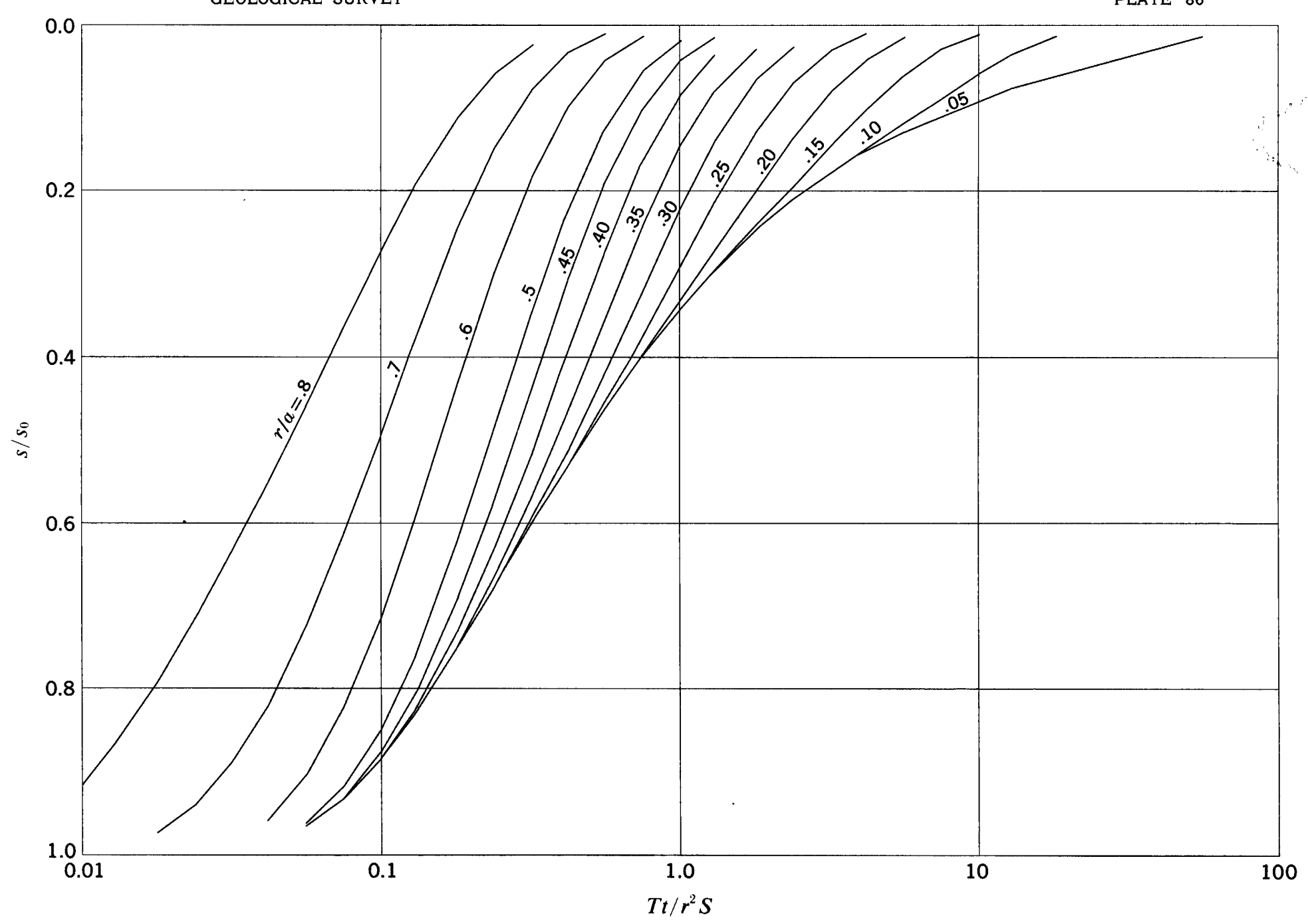

$s / s_{0}$ versus $T t / r^{2} S$ for $\theta_{0}=150^{\circ} ; \theta / \theta_{0}=0.30$ 
UNITED STATES DEPARTMENT OF THE INTERIOR GEOLOGICAL SURVEY

PROFESSIONAL PAPER 514 PLATE 87

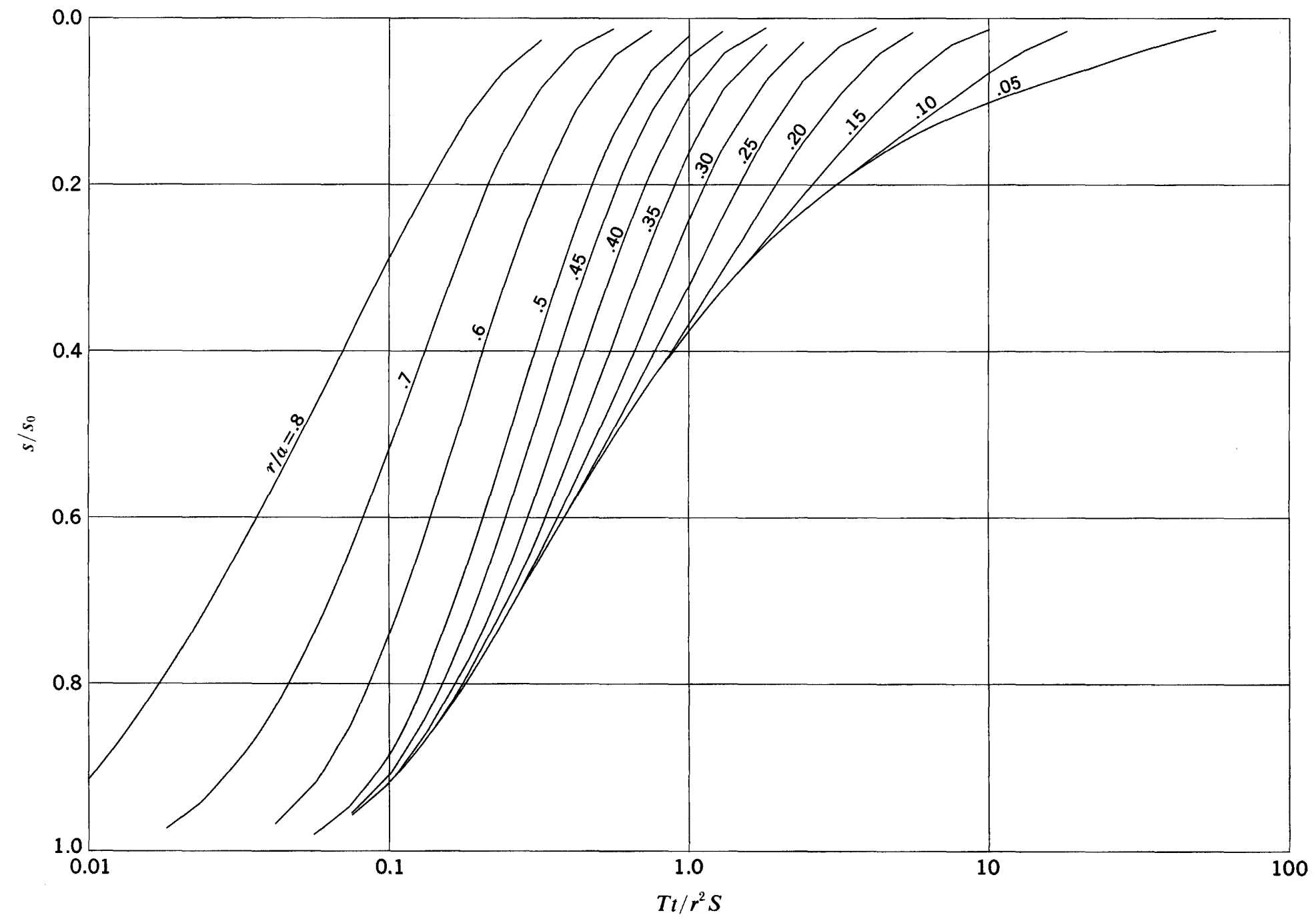

$s / s_{0}$ versus $T t / r^{2} S$ for $\theta_{0}=150^{\circ} ; \theta / \theta_{0}=0.35$ 


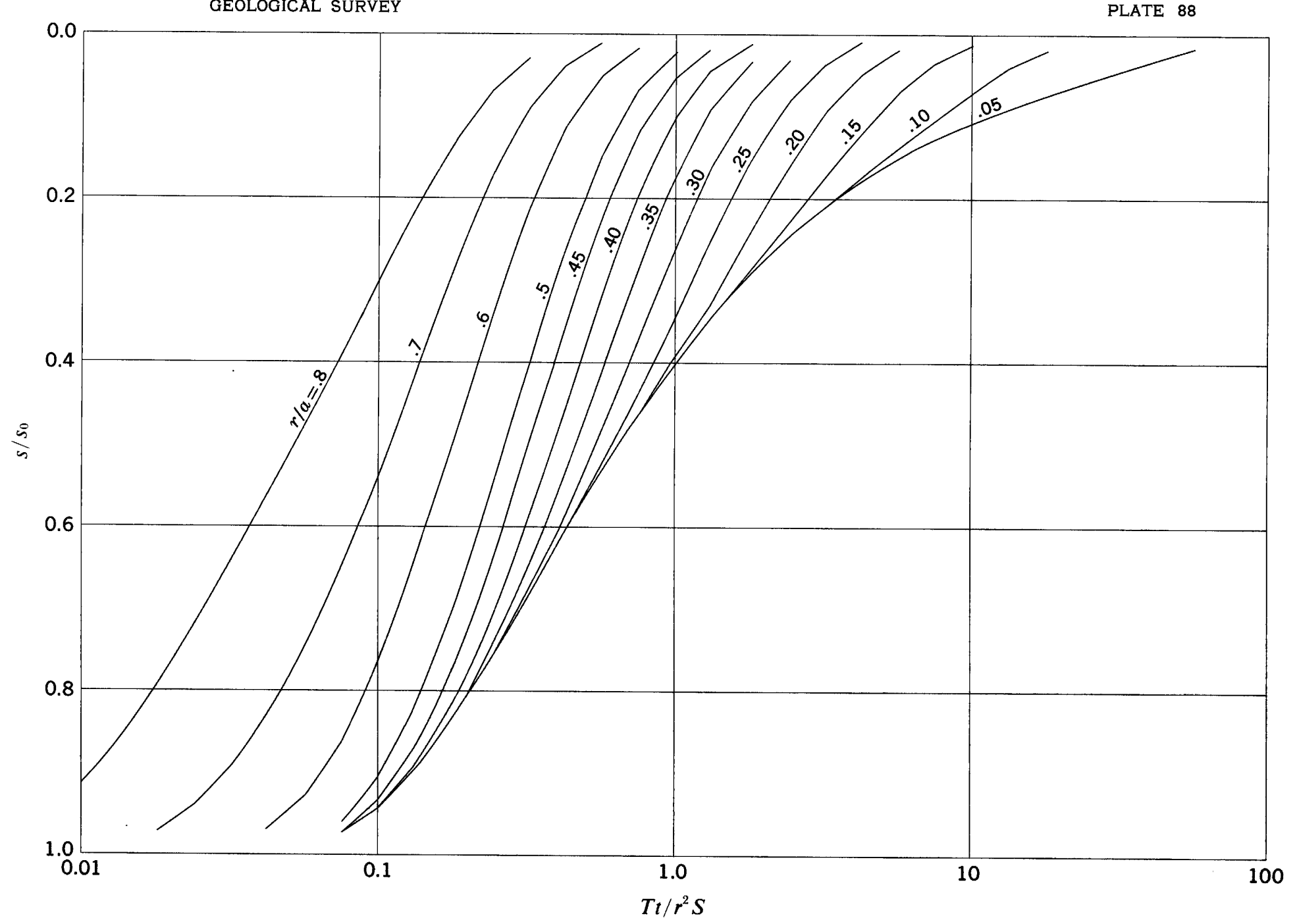

$s / s_{0}$ versus $T t / r^{2} S$ for $\theta_{0}=150^{\circ} ; \theta / \theta_{0}=0.40$ 

PLATE 89

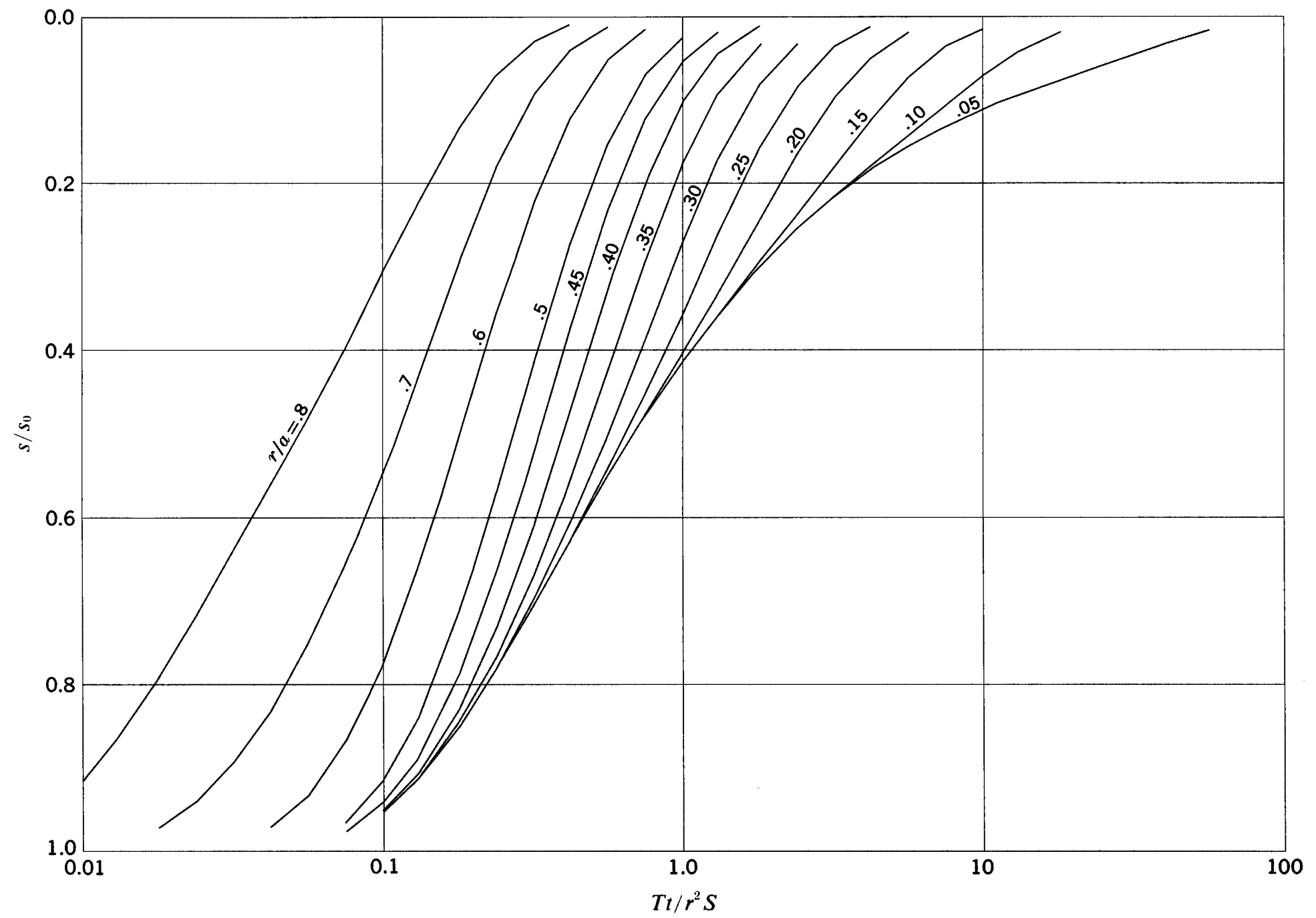

$s / s_{0}$ versus $T t / r^{2} S$ for $\theta_{0}=150^{\circ} ; \theta / \theta_{0}=0.45$ 


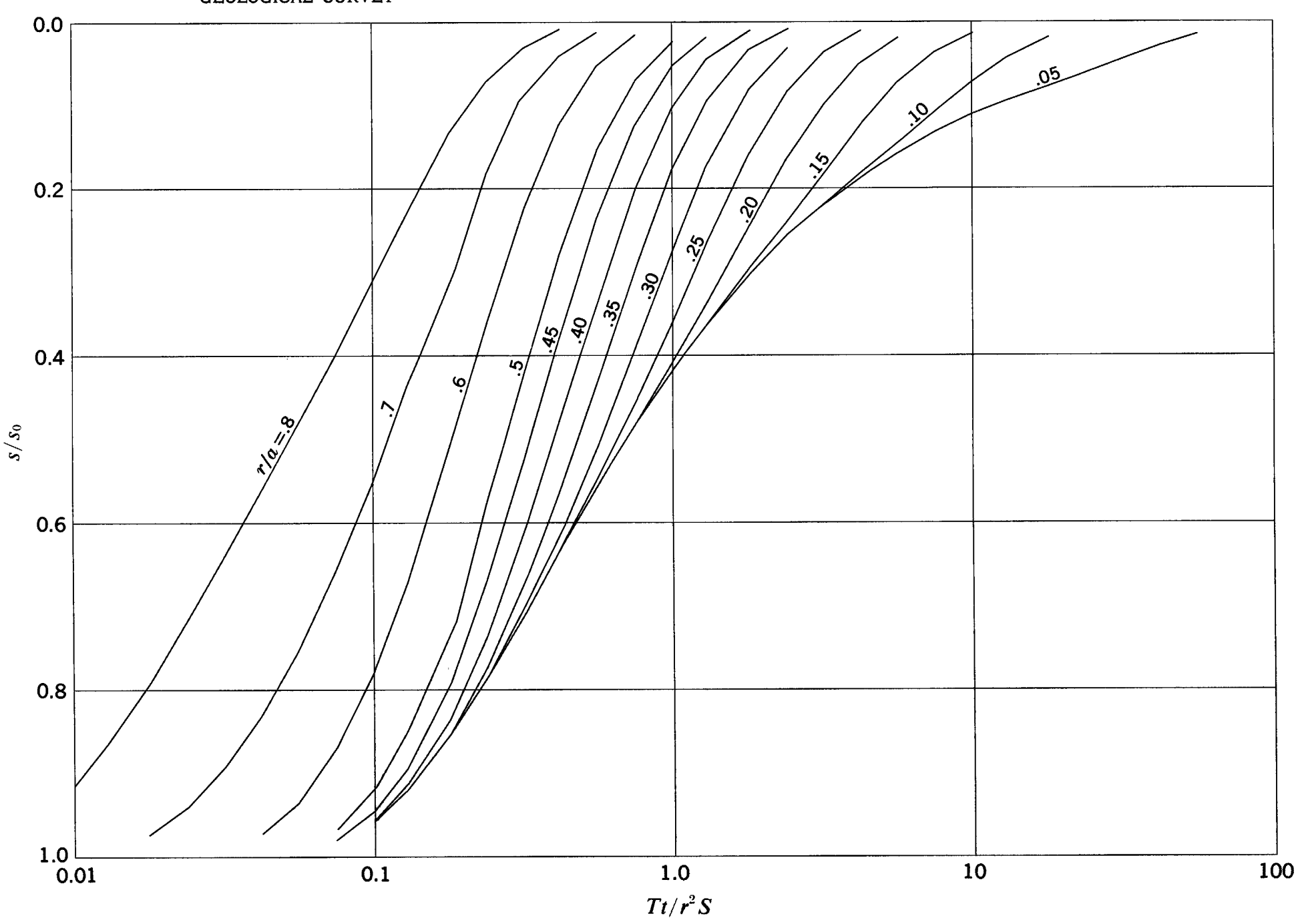

$s / s_{0}$ versus $T t / r^{2} S$ for $\theta_{0}=150^{\circ} ; \theta / \theta_{0}=0.50$ 
UNITED STATES DEPARTMENT OF THE INTERIOR GEOLOGICAL SURVEY
PROFESSIONAL PAPER 514

PLATE 91

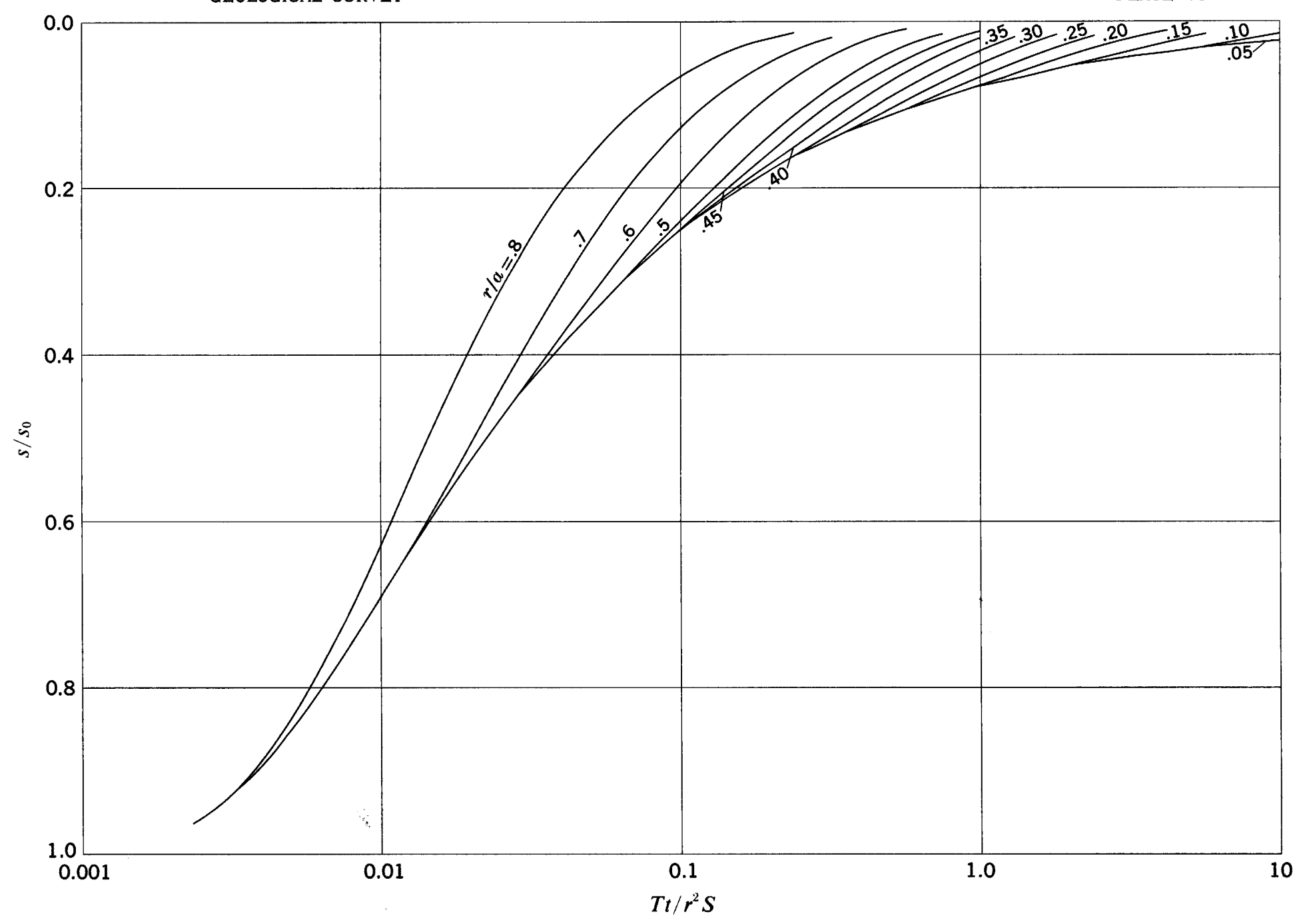

$s / s_{0}$ versus $T t / r^{2} S$ for $\theta_{0}=165^{\circ} ; \theta / \theta_{0}=0.05$ 


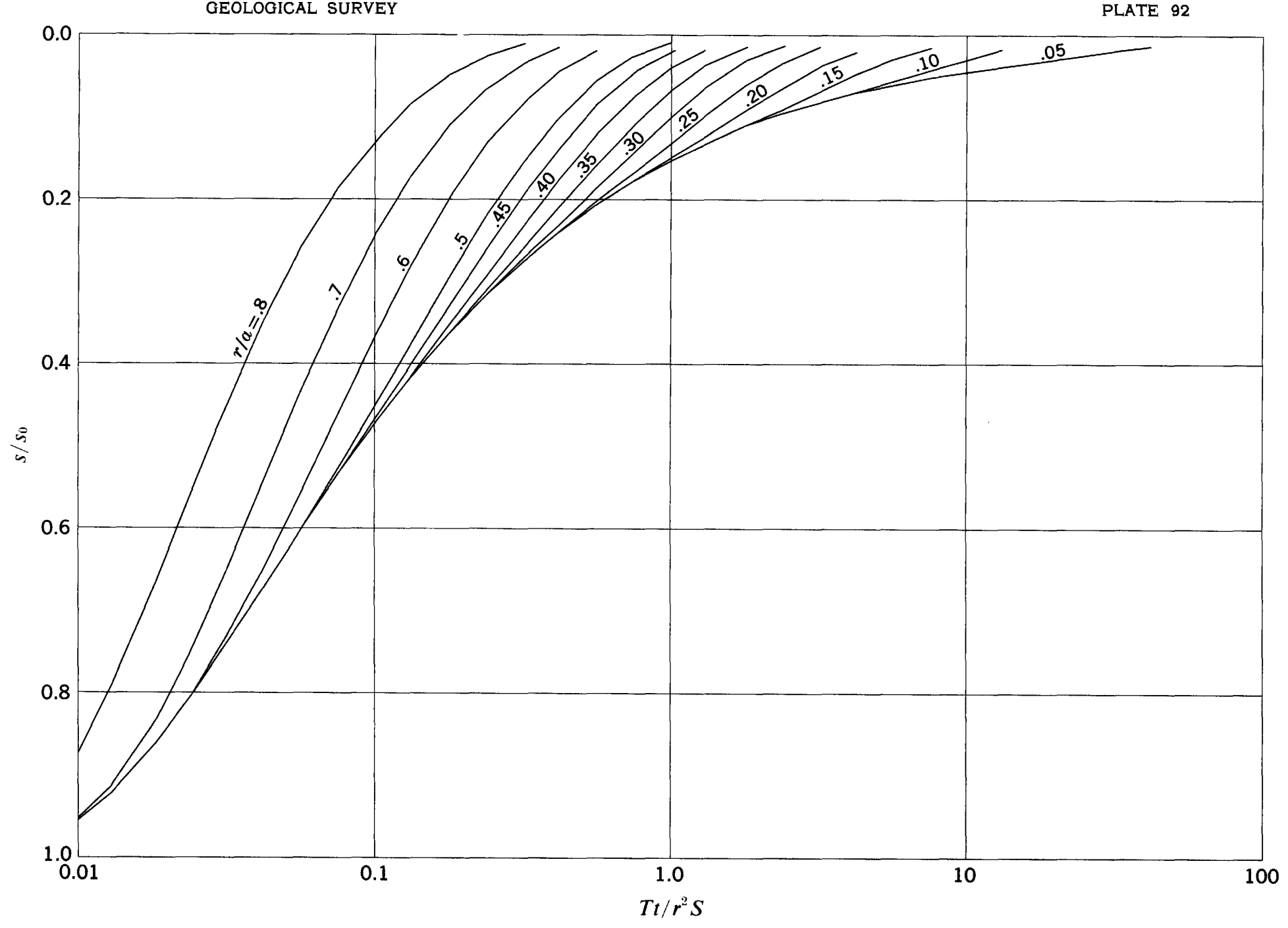

$s / s_{0}$ versus $T t / r^{2} S$ for $\theta_{0}=165^{\circ} ; \theta / \theta_{0}=0.10$ 
UNITED STATES DEPARTMENT OF THE INTERIOR GEOLOGICAL SURVEY

PROFESSIONAL PAPER 514

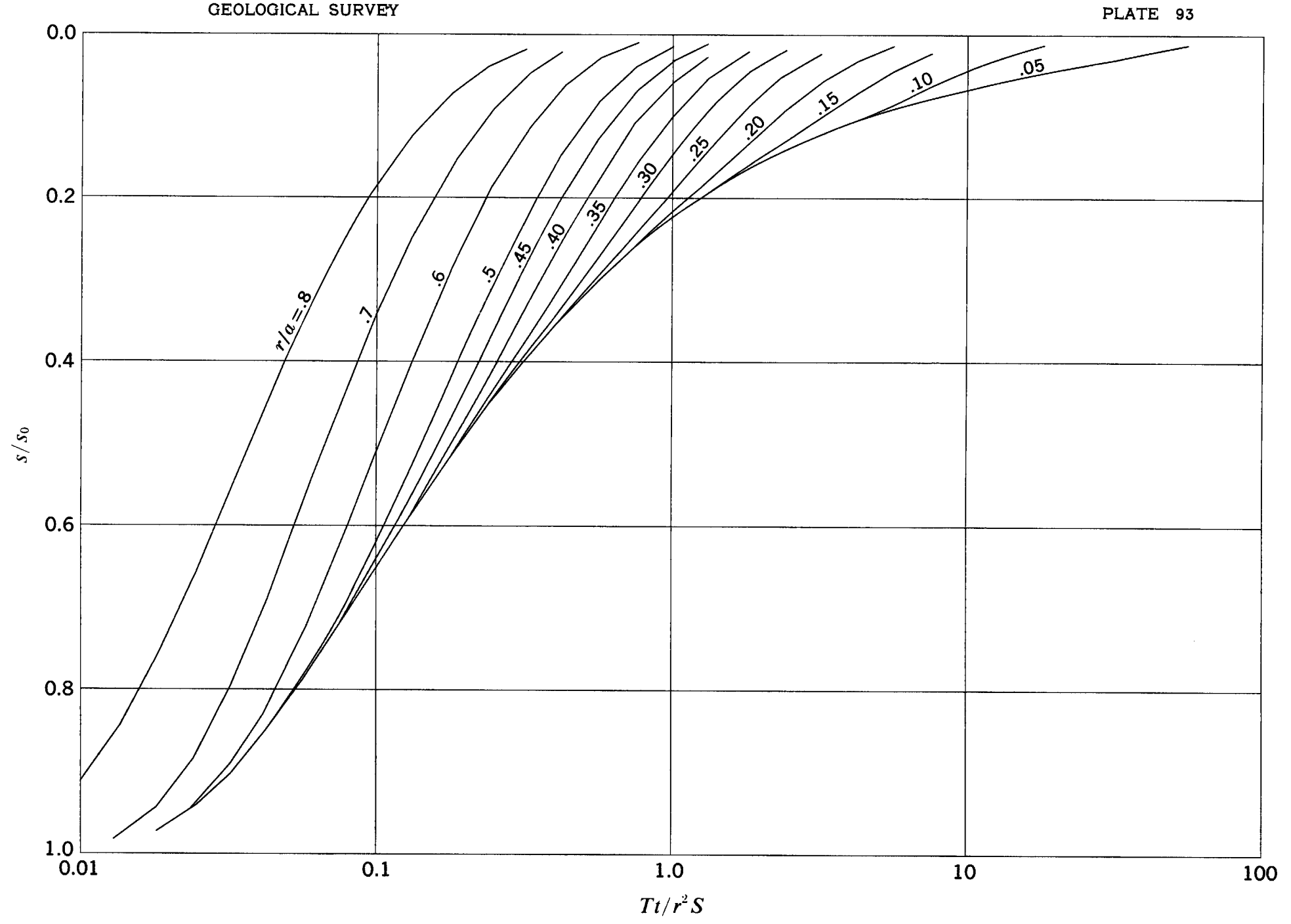

$s / s_{0}$ versus $T t / r^{2} S$ for $\theta_{0}=165^{\circ} ; \theta / \theta_{0}=0.15$ 
UNITED STATES DEPARTMENT OF THE INTERIOR GEOLOGICAL SURVEY
PROFESSIONAL PAPER 514 PLATE 94

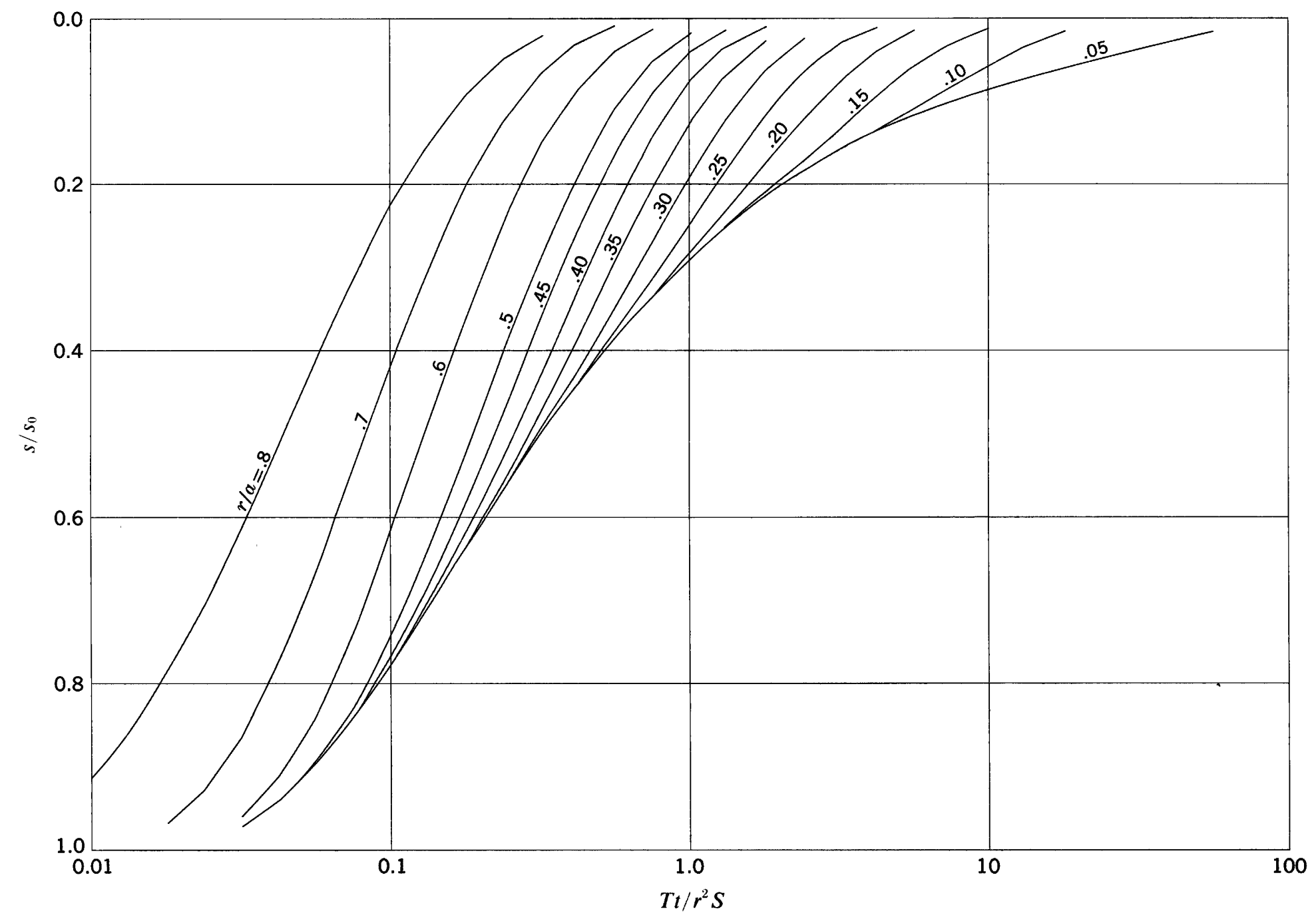

$s / s_{0}$ versus $T t / r^{2} S$ for $\theta_{0}=165^{\circ} ; \theta / \theta_{0}=0.20$ 
UNITED STATES DEPARTMENT OF THE INTERIOR GEOLOGICAL SURVEY
PROFESSIONAL PAPER 514 PLATE 95

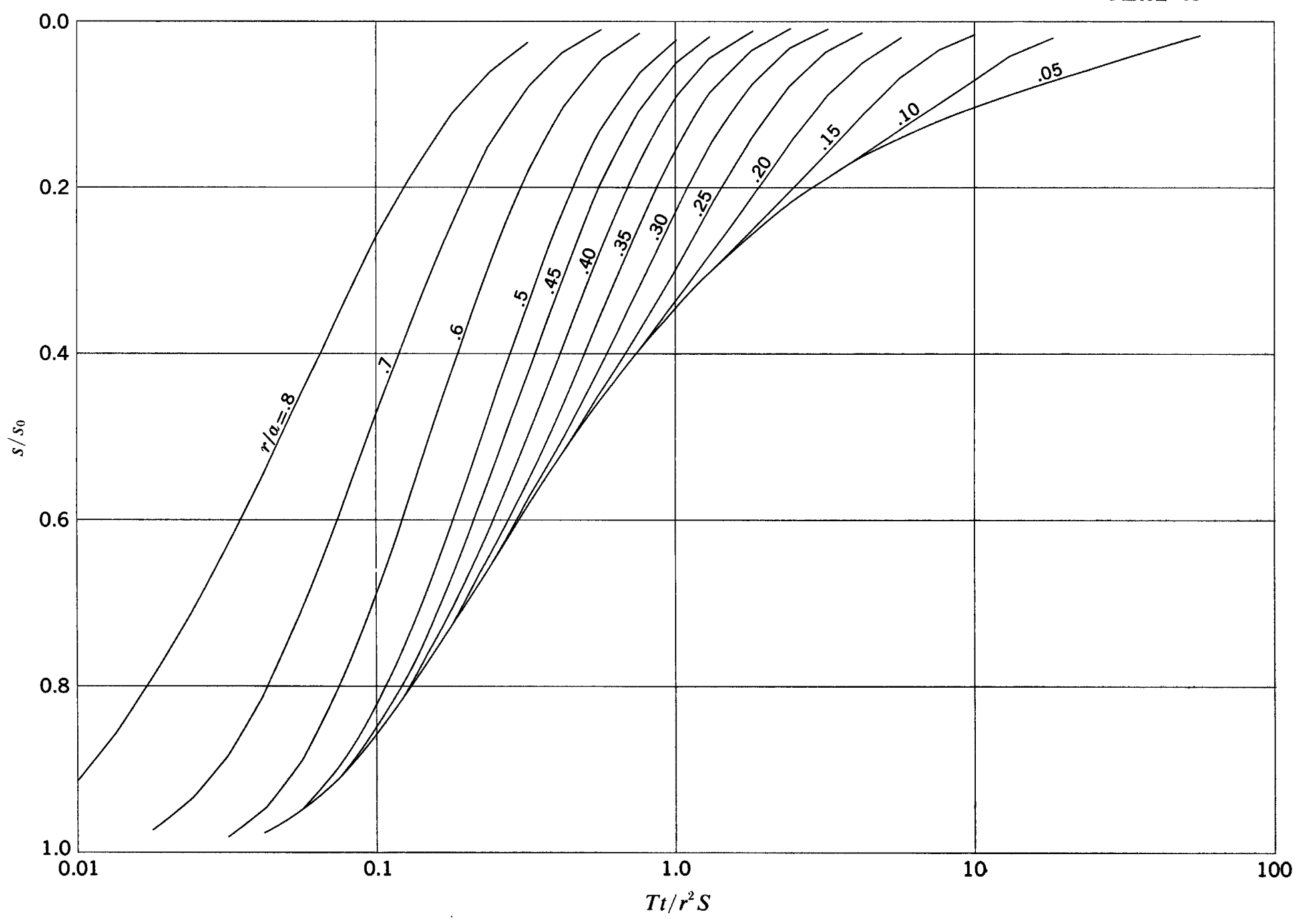

$s / s_{0}$ versus $T t / r^{2} S$ for $\theta_{0}=165^{\circ} ; \theta / \theta_{0}=0.25$ 

PLATE 96

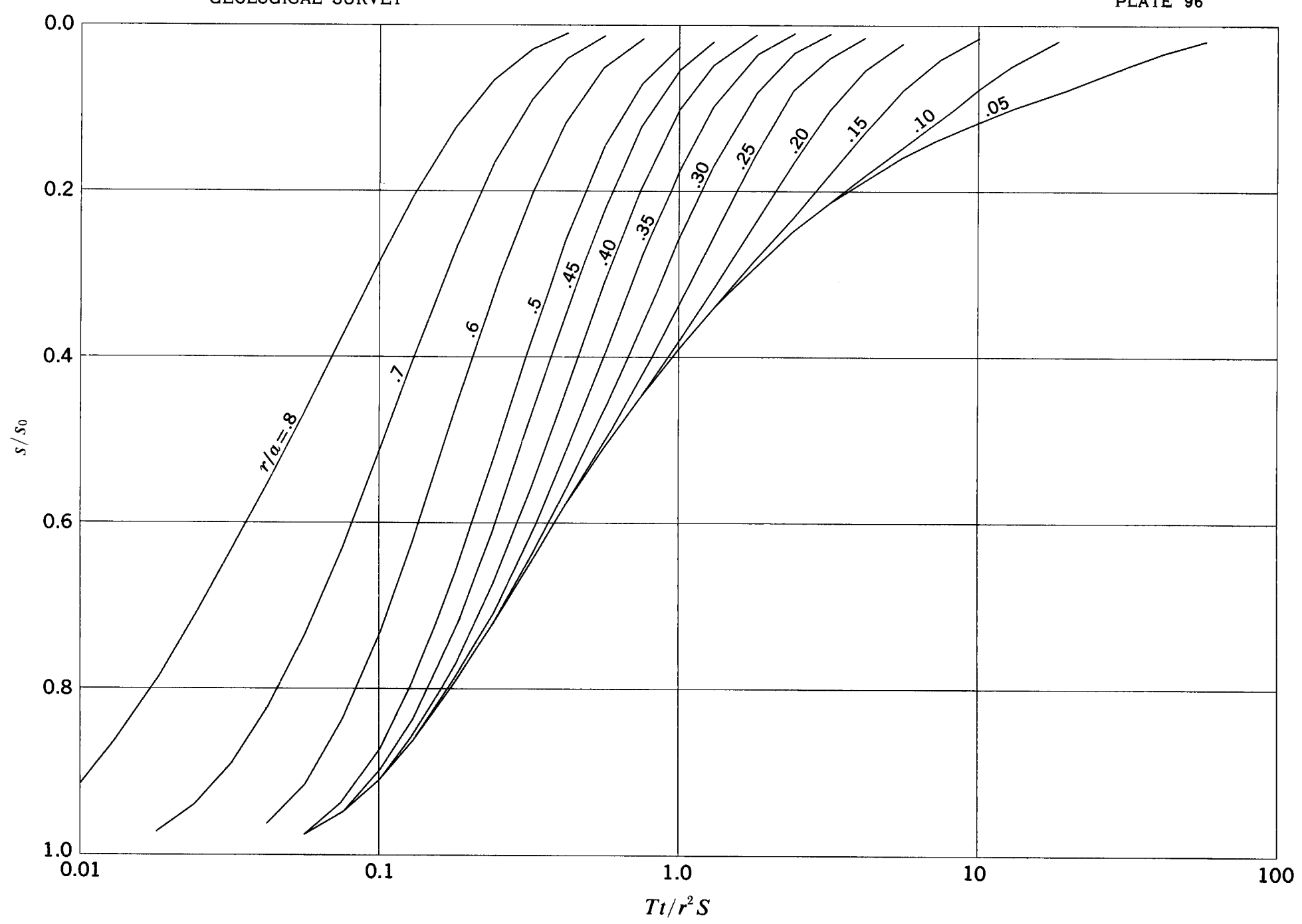

$s / s_{0}$ versus $T t / r^{2} S$ for $\theta_{0}=165^{\circ} ; \theta / \theta_{0}=0.30$ 


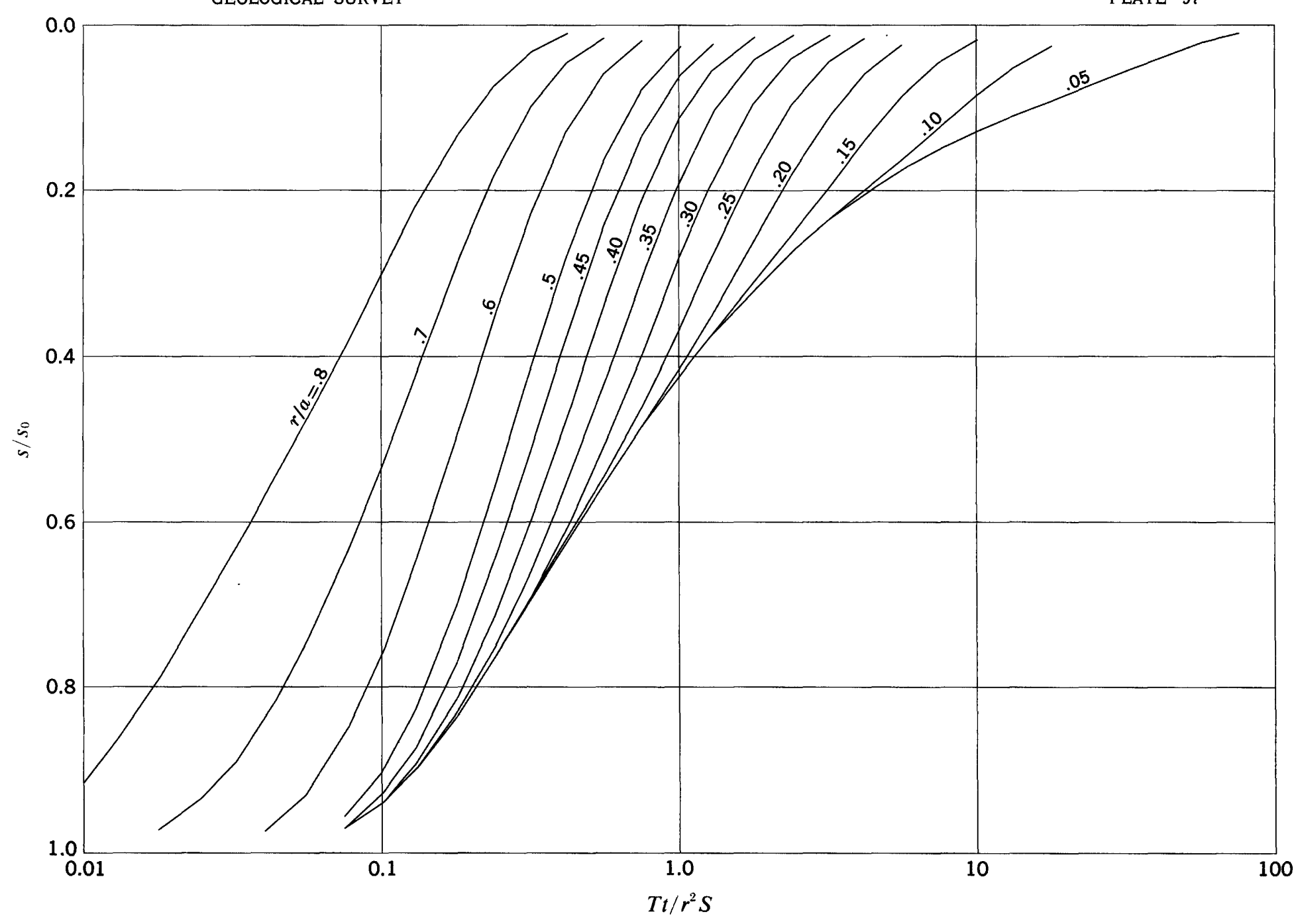

$s / s_{0}$ versus $T t / r^{2} S$ for $\theta_{0}=165^{\circ} ; \theta / \theta_{0}=0.35$ 


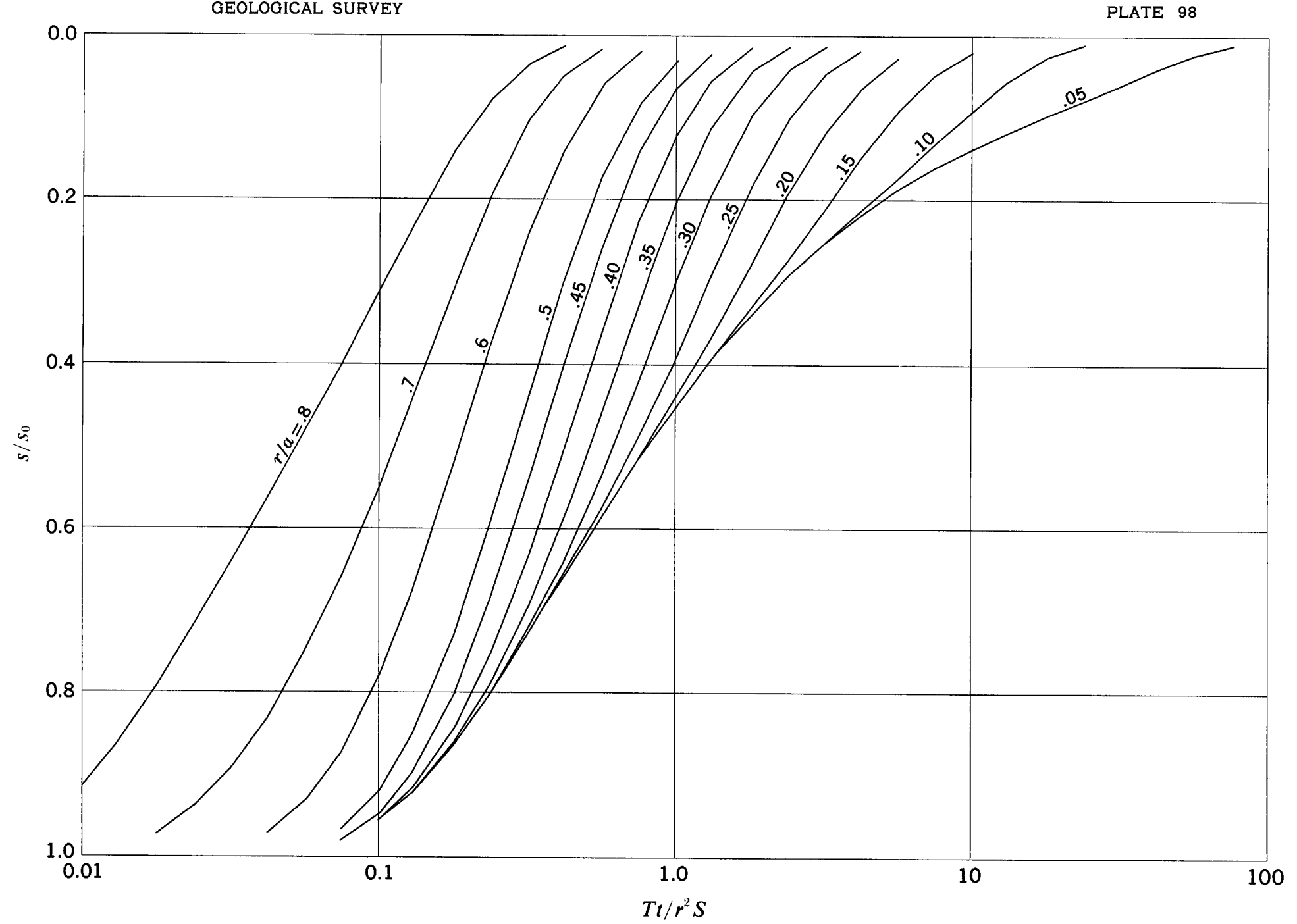

$s / s_{0}$ versus $T t / r^{2} S$ for $\theta_{0}=165^{\circ} ; \theta / \theta_{0}=0.40$ 

GEOLOGICAL SURVEY

PROFESSIONAL PAPER 514

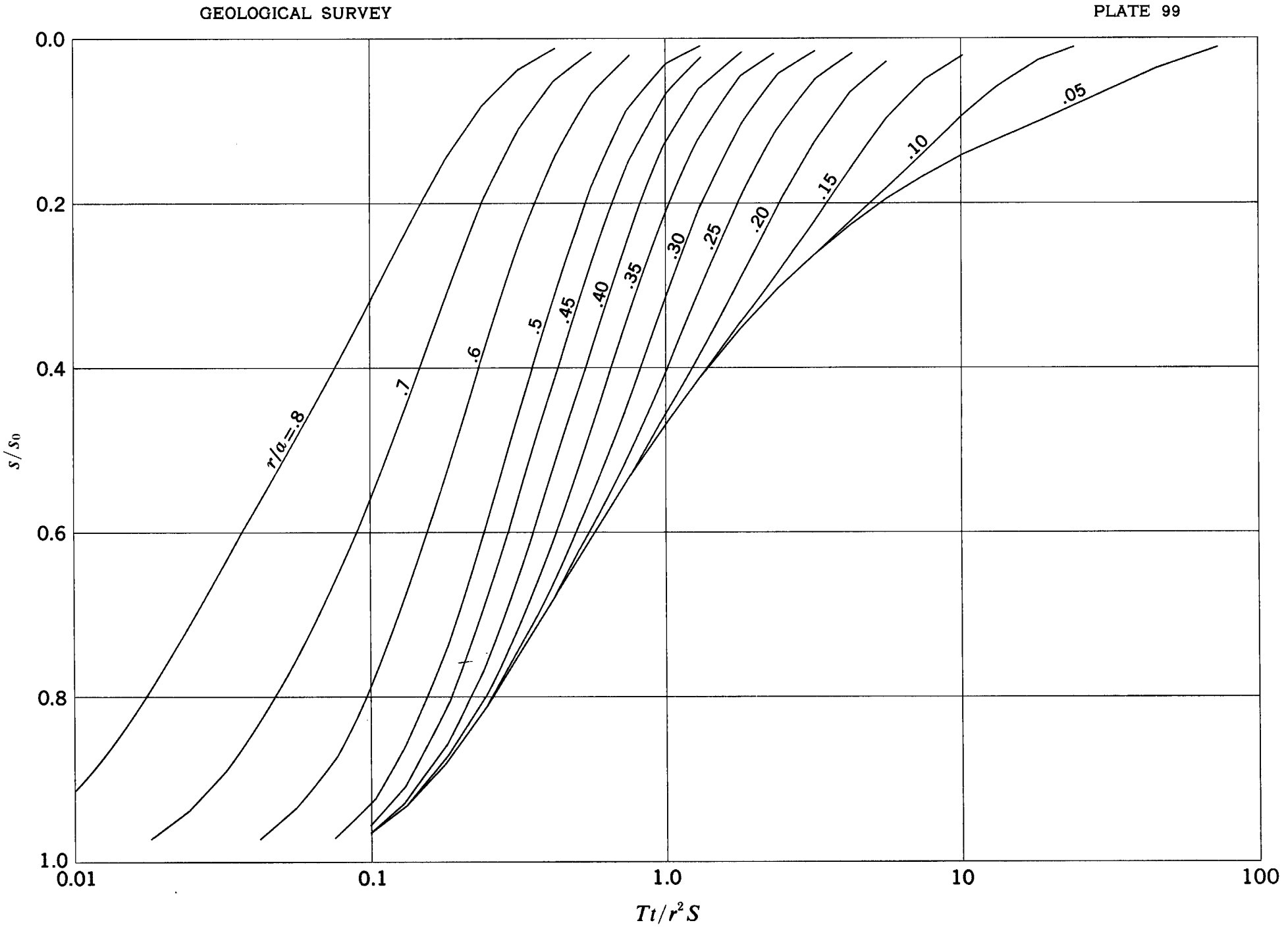

$s / s_{0}$ versus $T t / r^{2} S$ for $\theta_{0}=165^{\circ} ; \theta / \theta_{0}=0.45$ 
UNITED STATES DEPARTMENT OF THE INTERIOR GEOLOGICAL SURVEY

PROFESSIONAL PAPER 514 PLATE 100

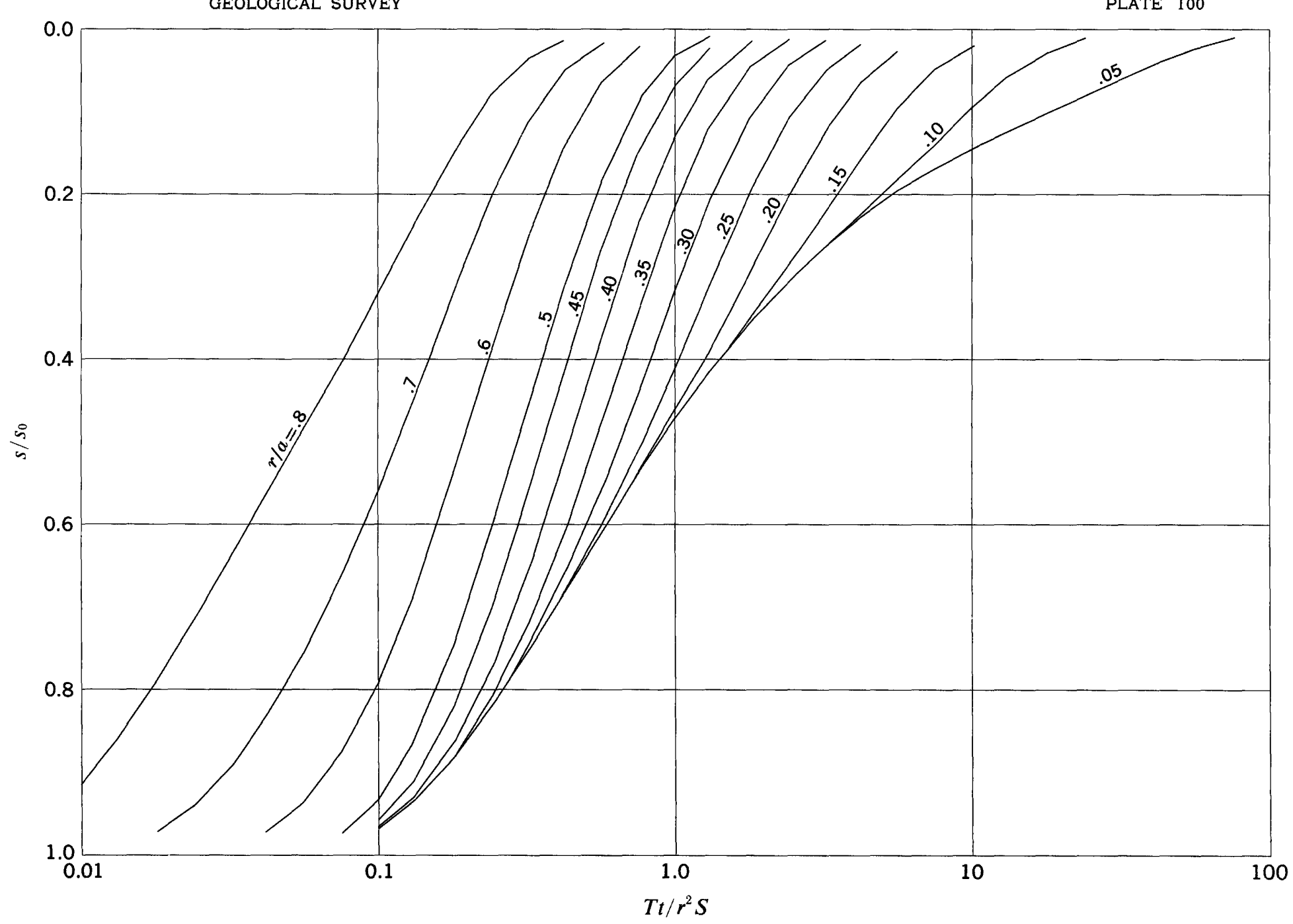

$s / s_{0}$ versus $T t / r^{2} S$ for $\theta_{0}=165^{\circ} ; \theta / \theta_{0}=0.50$ 


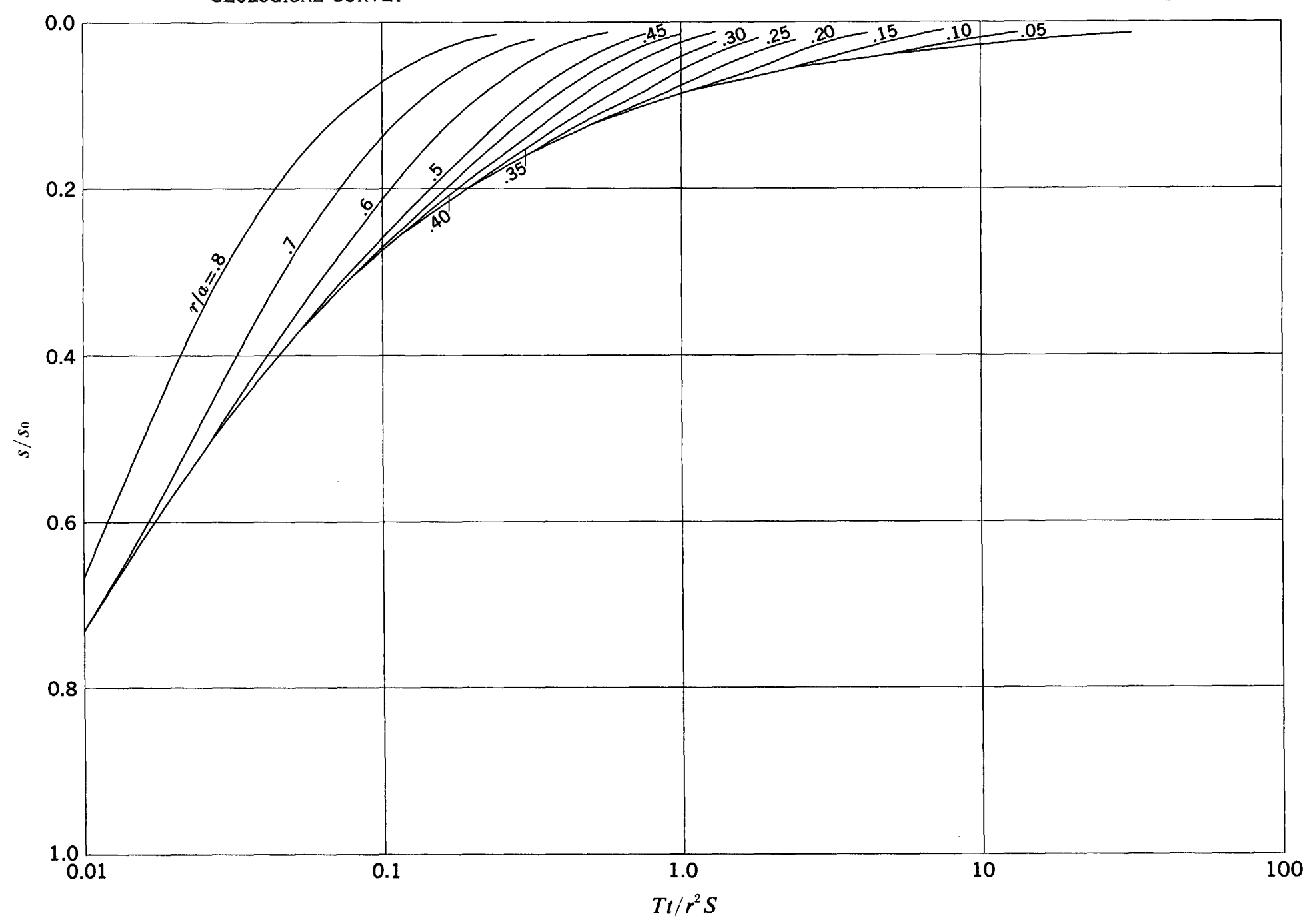

$s / s_{0}$ versus $T t / r^{2} S$ for $\theta_{0}=180^{\circ} ; \theta / \theta_{0}=0.05$ 
UNITED STATES DEPARTMENT OF THE INTERIOR GEOLOGICAL SURVEY
PROFESSIONAL PAPER 514 PLATE 102

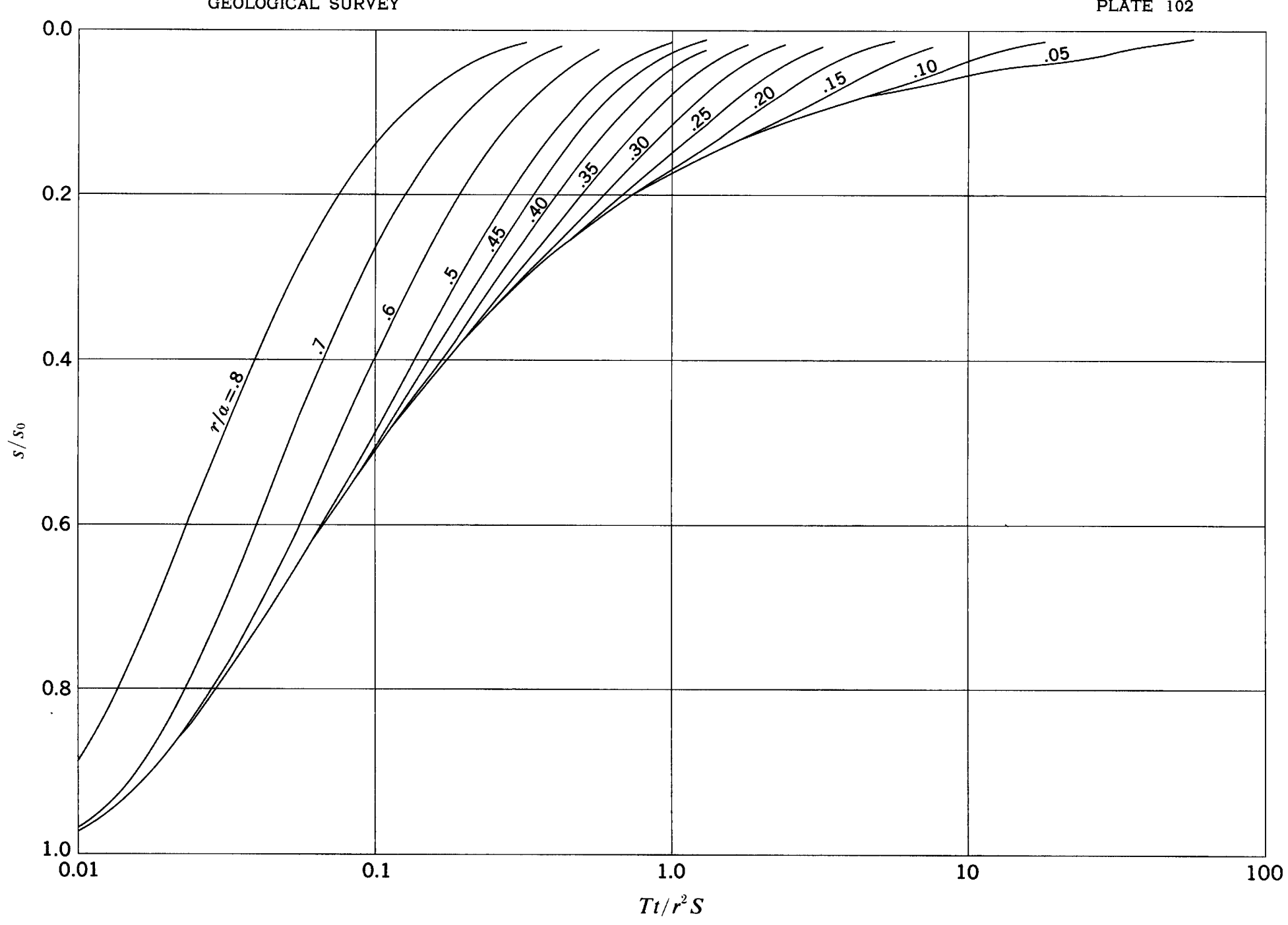

$s / s_{0}$ versus $T t / r^{2} S$ for $\theta_{0}=180^{\circ} ; \theta / \theta_{0}=0.10$ 
UNITED STATES DEPARTMENT OF THE INTERIOR GEOLOGICAL SURVEY
PROFESSIONAL PAPER 514 PLATE 103

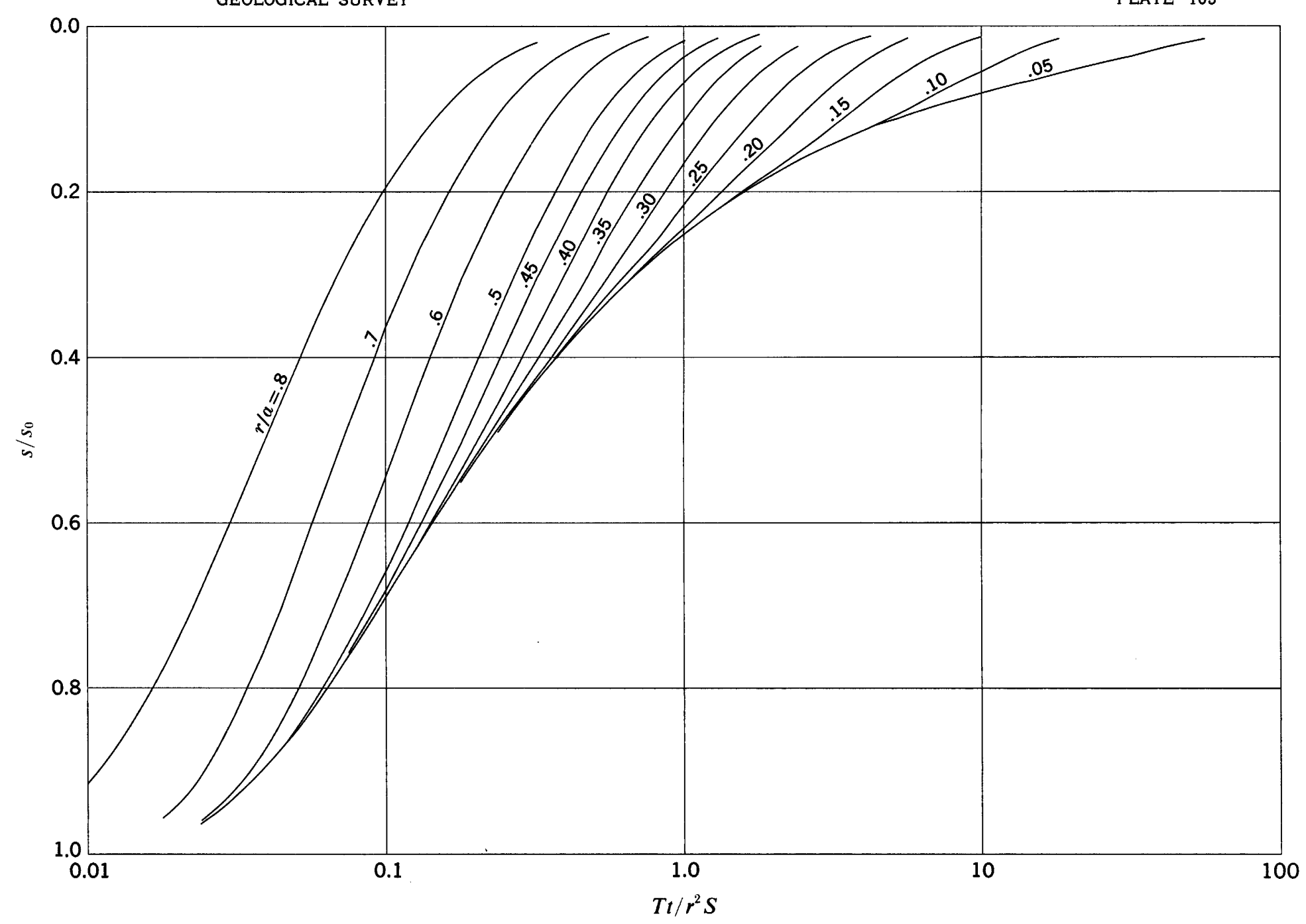

$s / s_{0}$ versus $T t / r^{2} S$ for $\theta_{0}=180^{\circ} ; \theta / \theta_{0}=0.15$ 


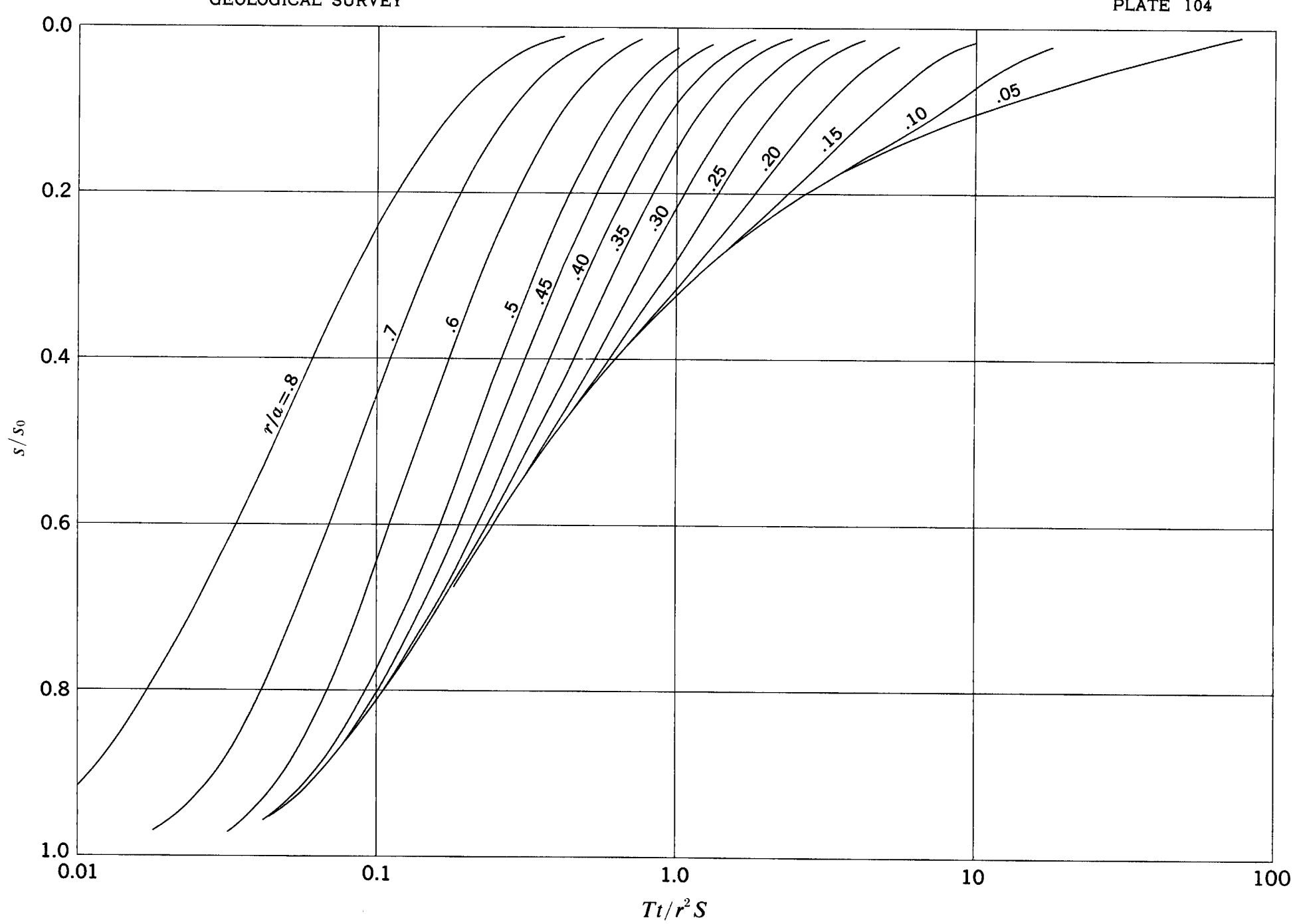

$s / s_{0}$ versus $T t / r^{2} S$ for $\theta_{0}=180^{\circ} ; \theta / \theta_{0}=0.20$ 

GEOLOGICAL SURVEY PLATE 105

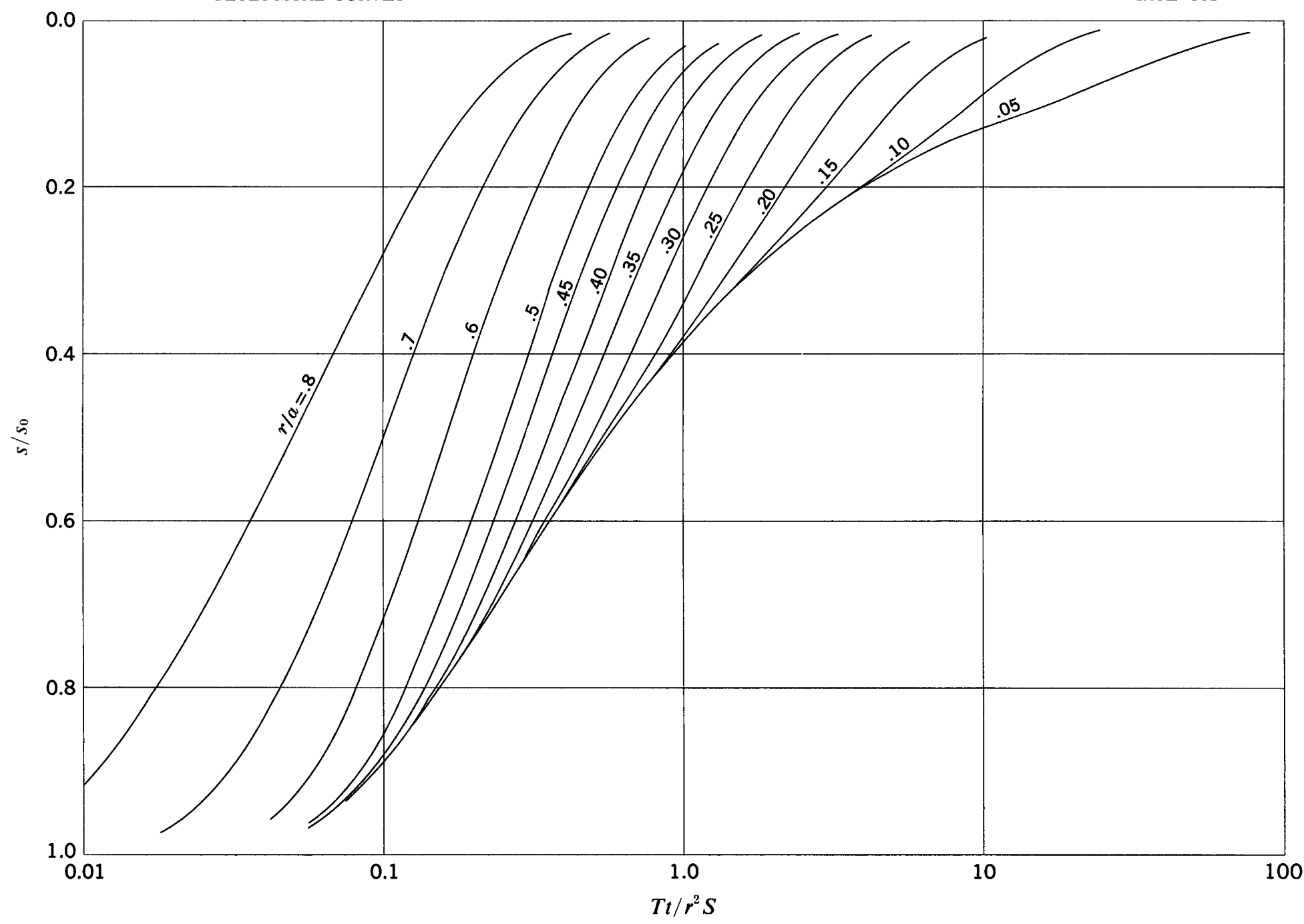

$s / s_{0}$ versus $T t / r^{2} S$ for $\theta_{0}=180^{\circ} ; \theta / \theta_{0}=0.25$ 


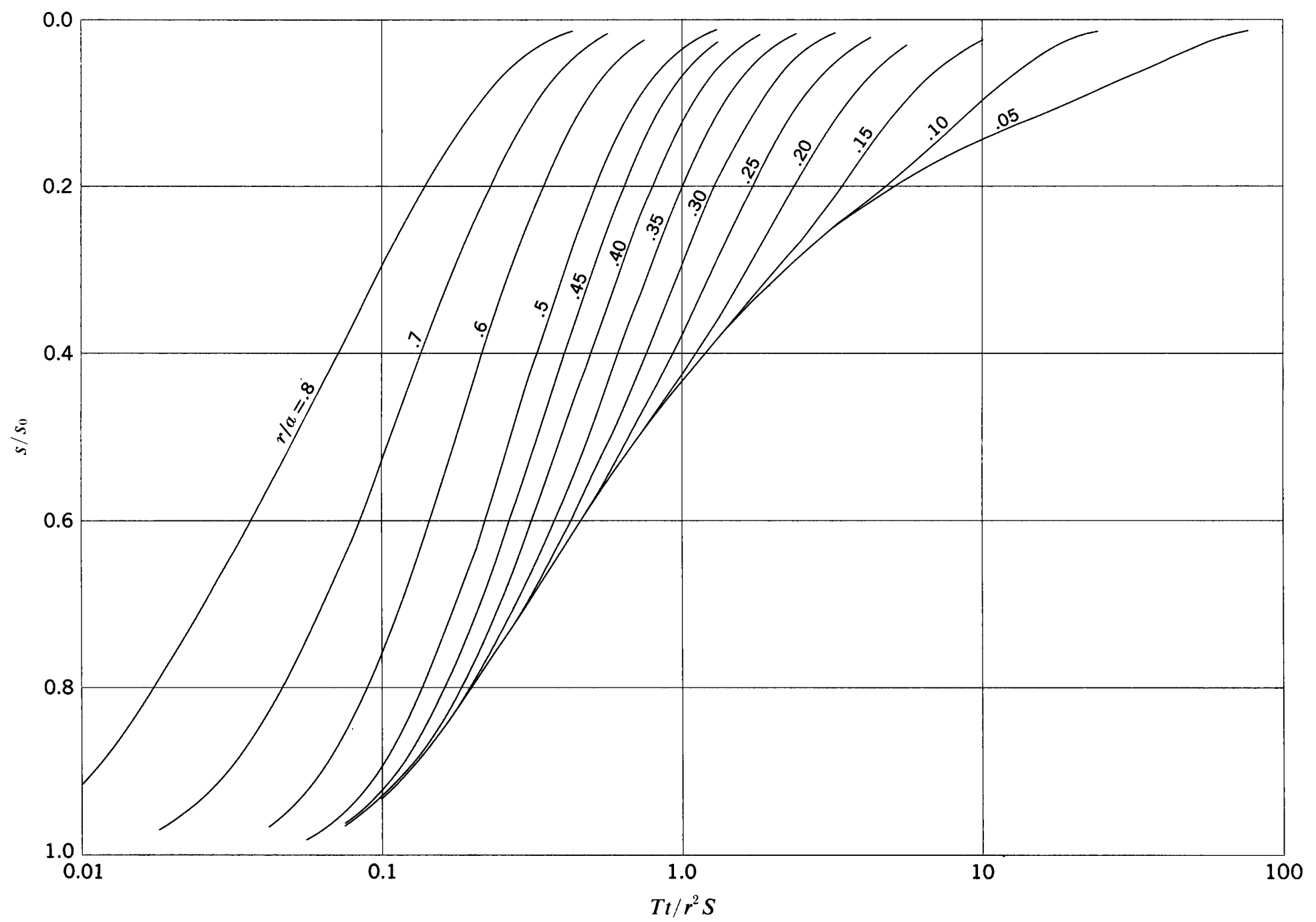

$s / s_{0}$ versus $T t / r^{2} S$ for $\theta_{0}=180^{\circ} ; \theta / \theta_{0}=0.30$ 


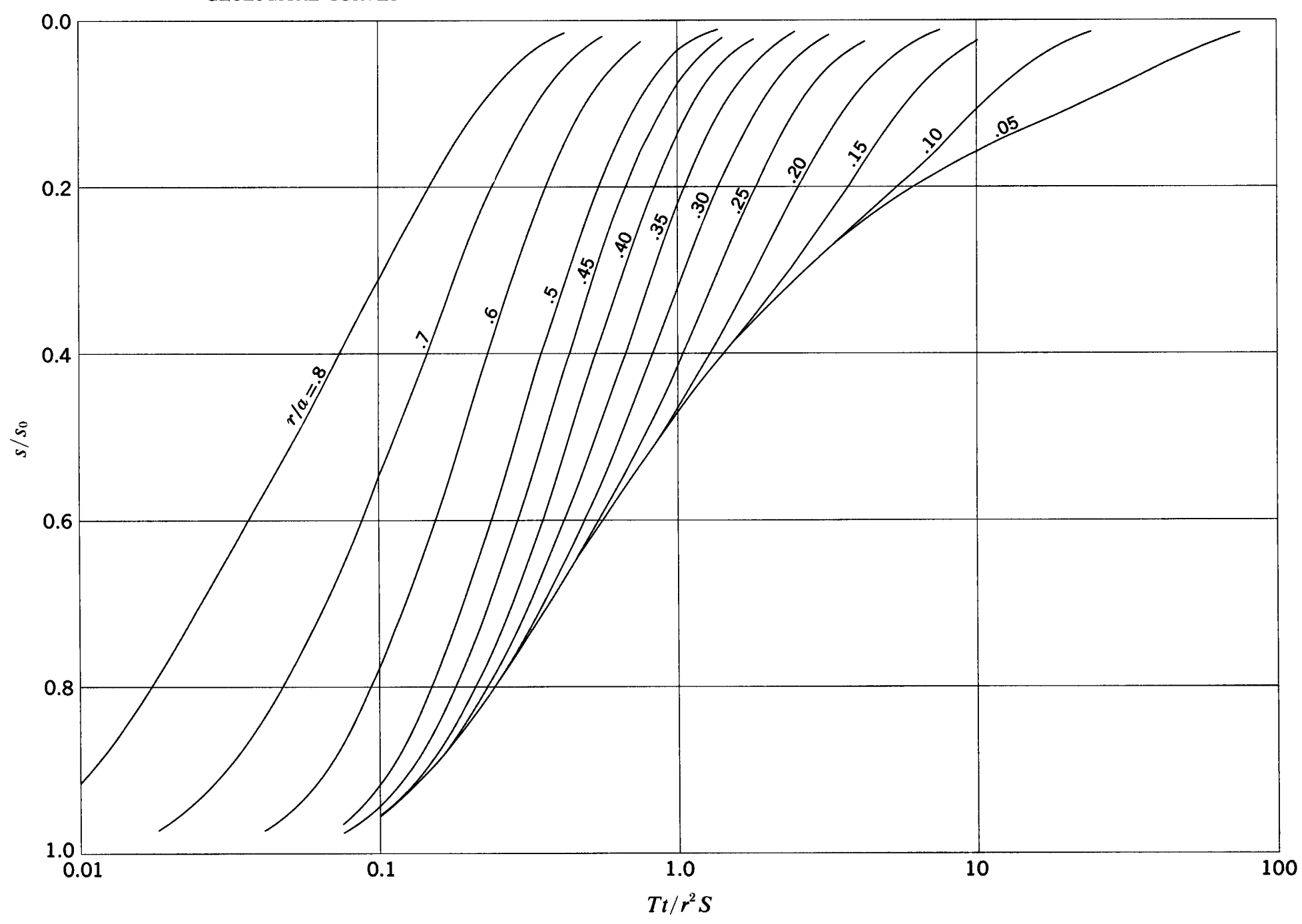

$s / s_{0}$ versus $T t / r^{2} S$ for $\theta_{0}=180^{\circ} ; \theta / \theta_{0}=0.35$ 

GEOLOGICAL SURVEY

PROFESSIONAL PAPER 514

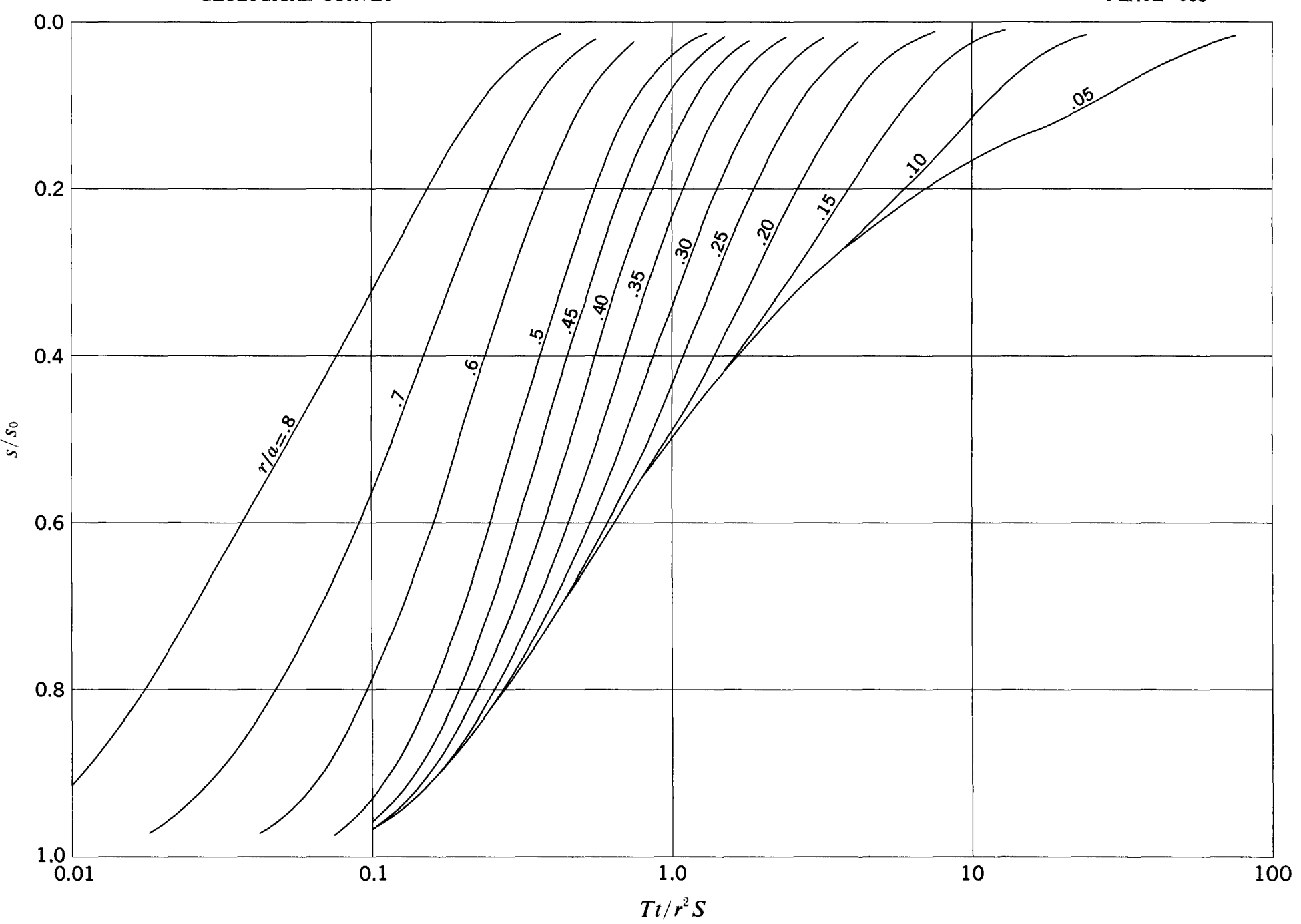

$s / s_{0}$ versus $T t / r^{2} S$ for $\theta_{0}=180^{\circ} ; \theta / \theta_{0}=0.40$ 


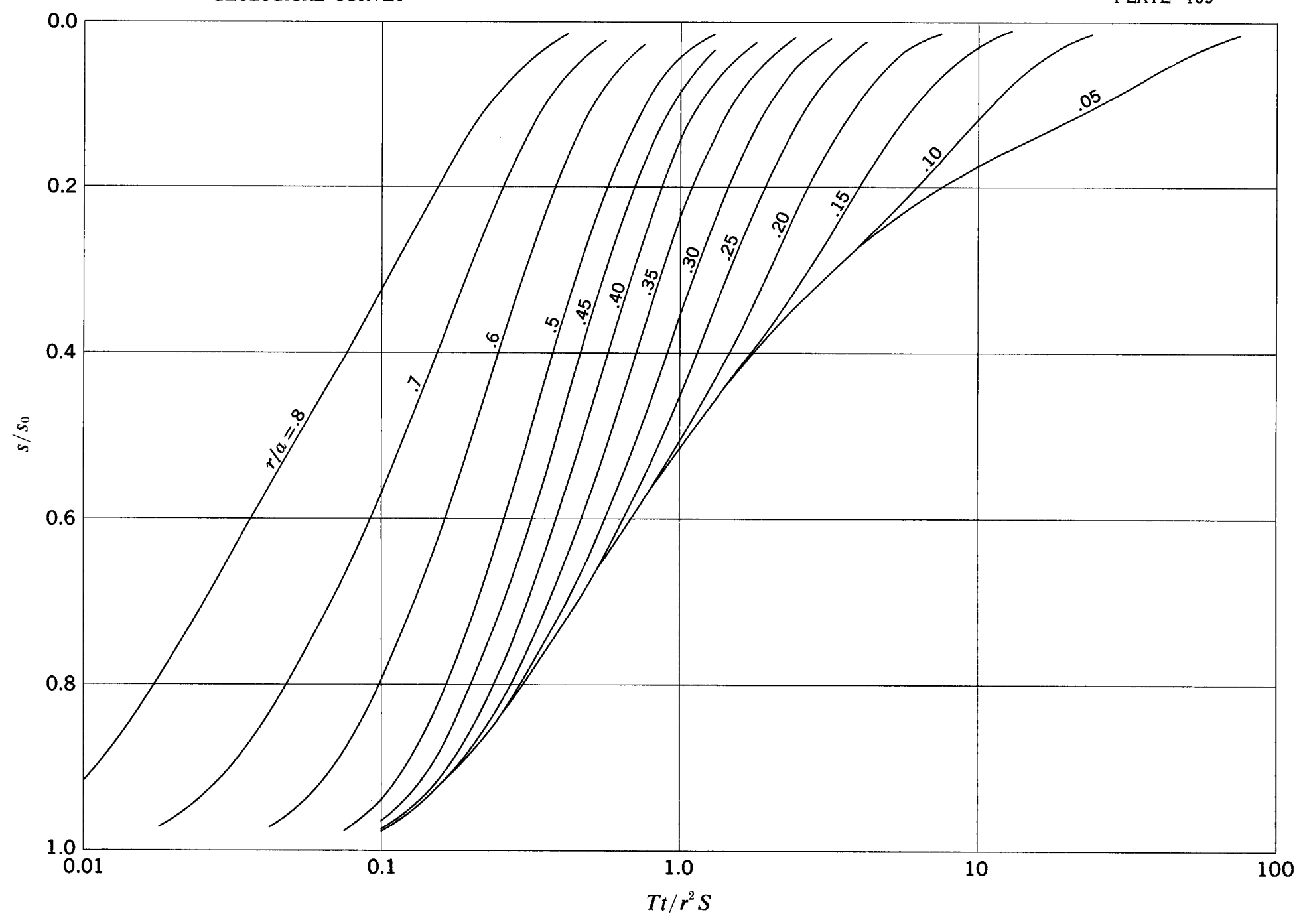

$s / s_{0}$ versus $T t / r^{2} S$ for $\theta_{0}=180^{\circ} ; \theta / \theta_{0}=0.45$ 


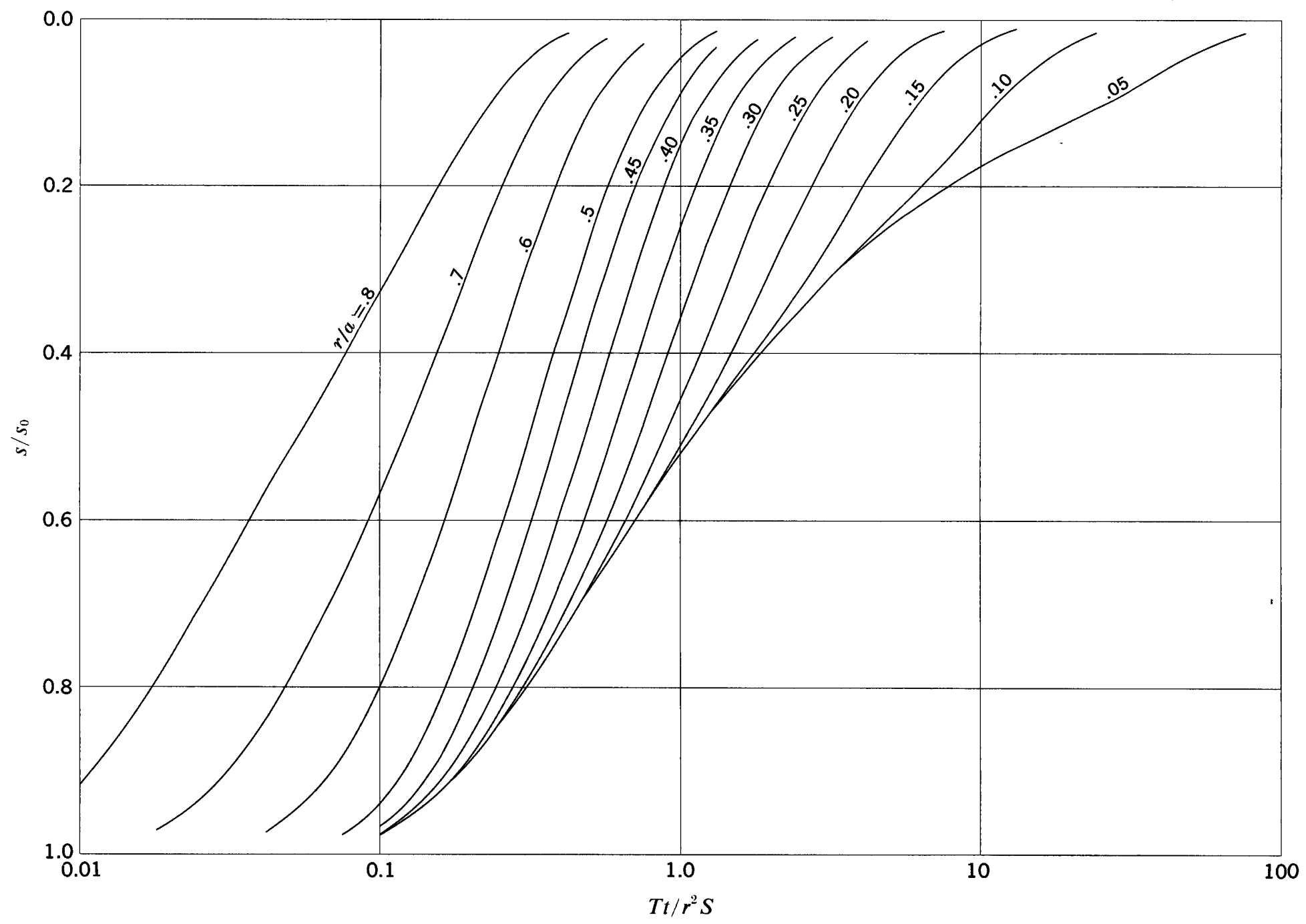

$s / s_{0}$ versus $T t / r^{2} S$ for $\theta_{0}=180^{\circ} ; \theta / \theta_{0}=0.50$ 

GEOLOGICAL SURVEY

PLATE 111

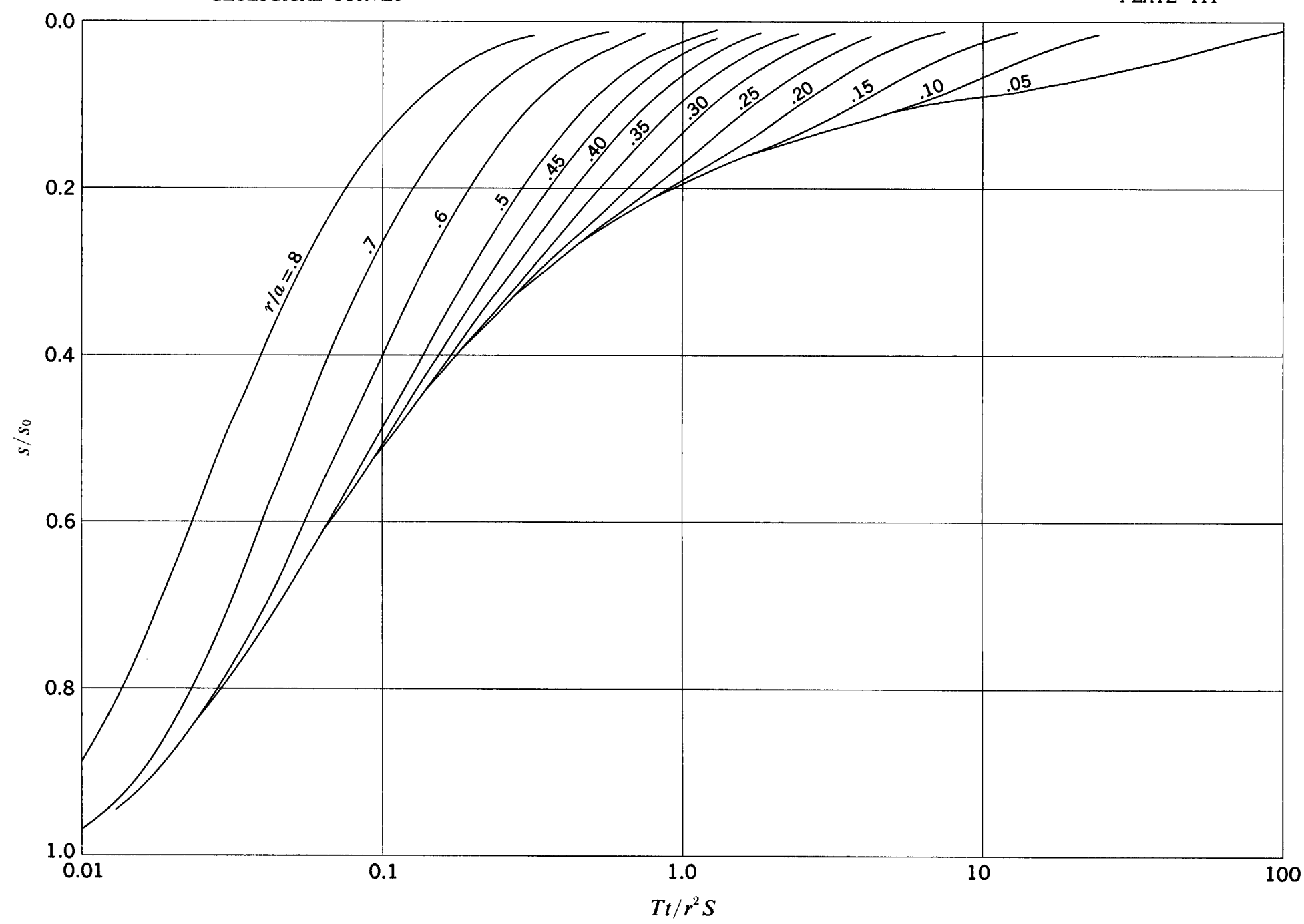

$s / s_{0}$ versus $T t / r^{2} S$ for $\theta_{0}=360^{\circ} ; \theta / \theta_{0}=0.05$ 


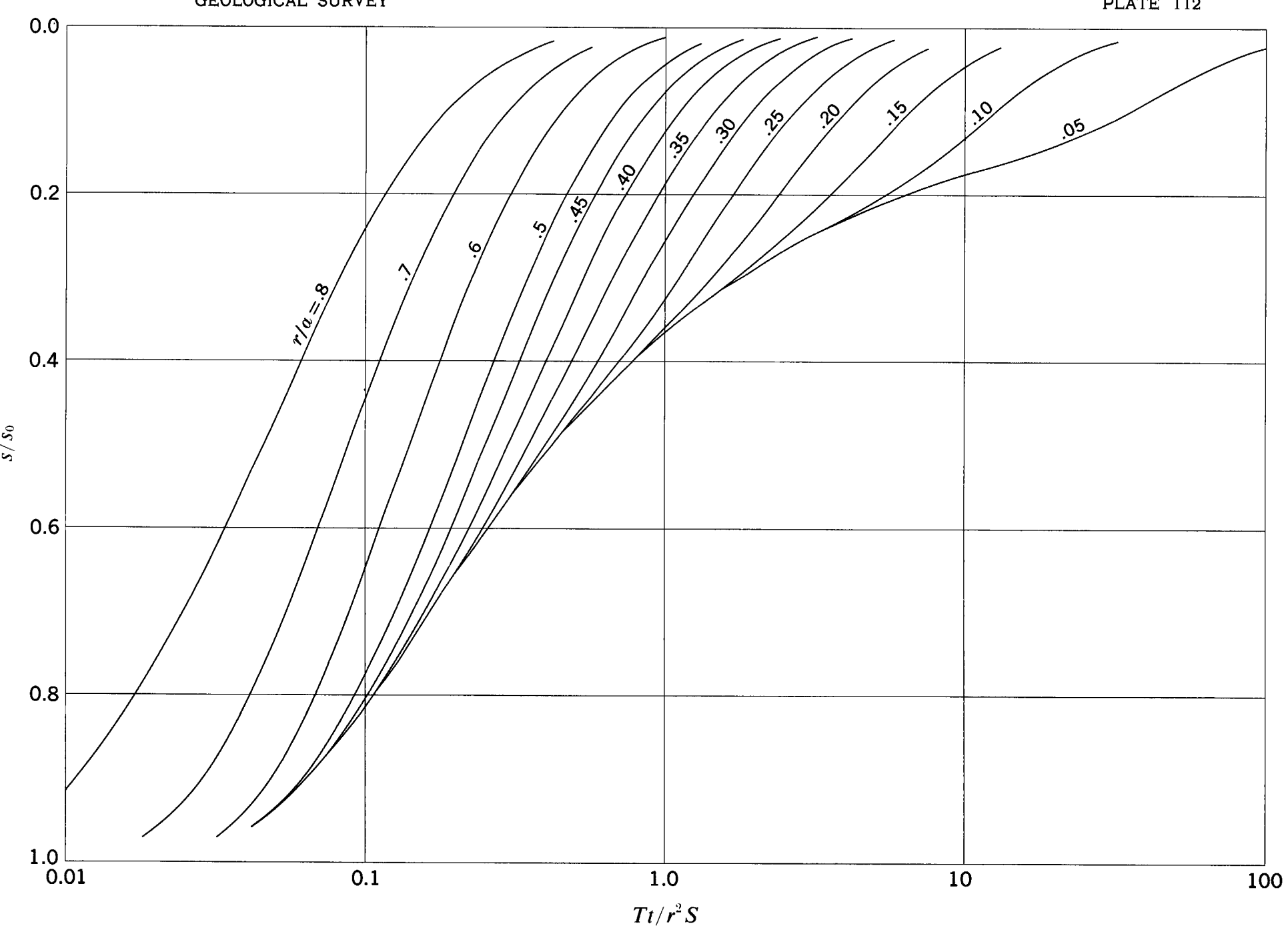

$s / s_{0}$ versus $T t / r^{2} S$ for $\theta_{0}=360^{\circ} ; \theta / \theta_{0}=0.10$ 
UNITED STATES DEPARTMENT OF THE INTERIOR GEOLOGICAL SURVEY
PROFESSIONAL PAPER 514 PLATE 113

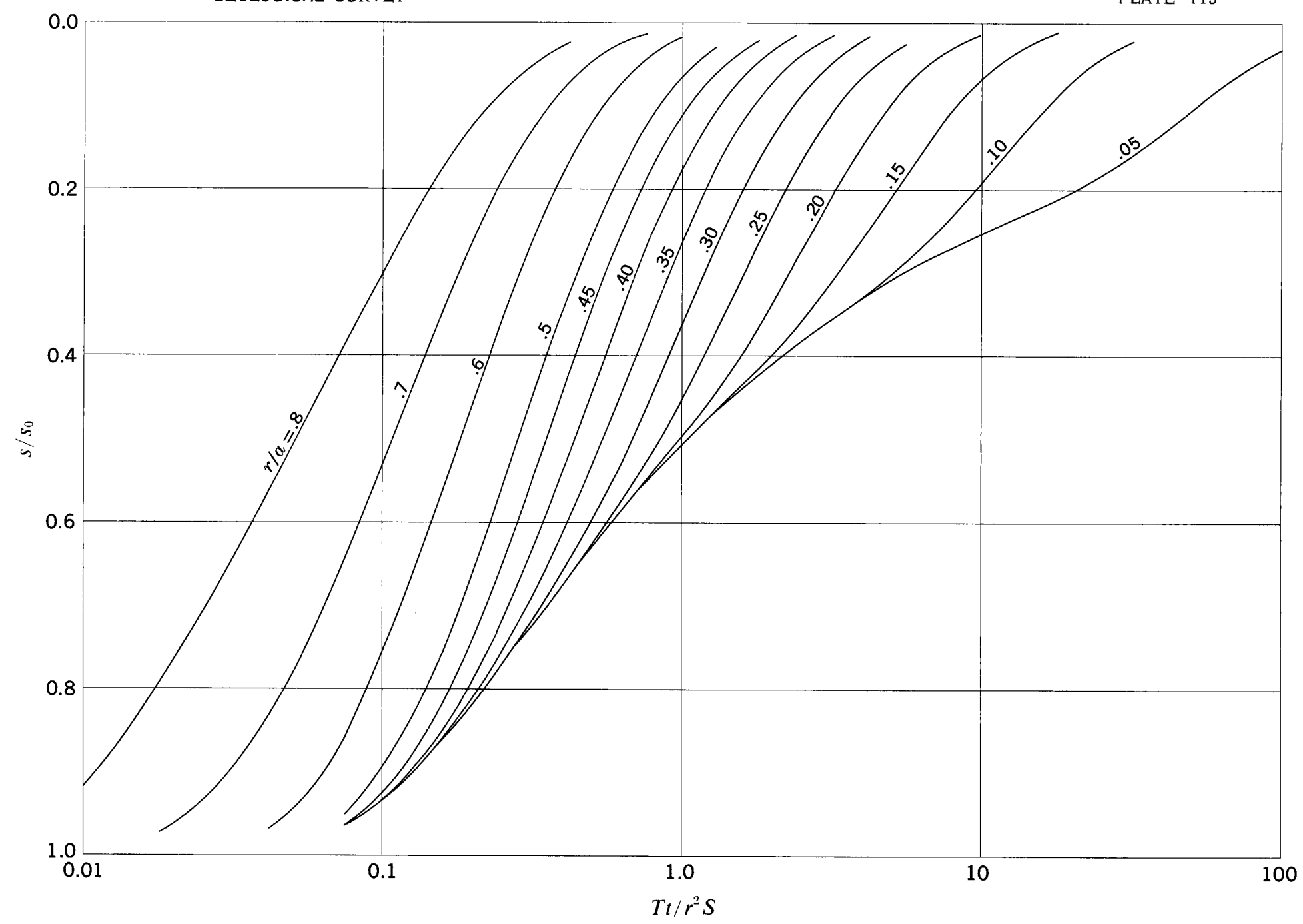

$s / s_{0}$ versus $T t / r^{2} S$ for $\theta_{0}=360^{\circ} ; \theta / \theta_{0}=0.15$ 
UNITED STATES DEPARTMENT OF THE INTERIOR GEOLOGICAL SURVEY

PROFESSIONAL PAPER 514 PLATE 114

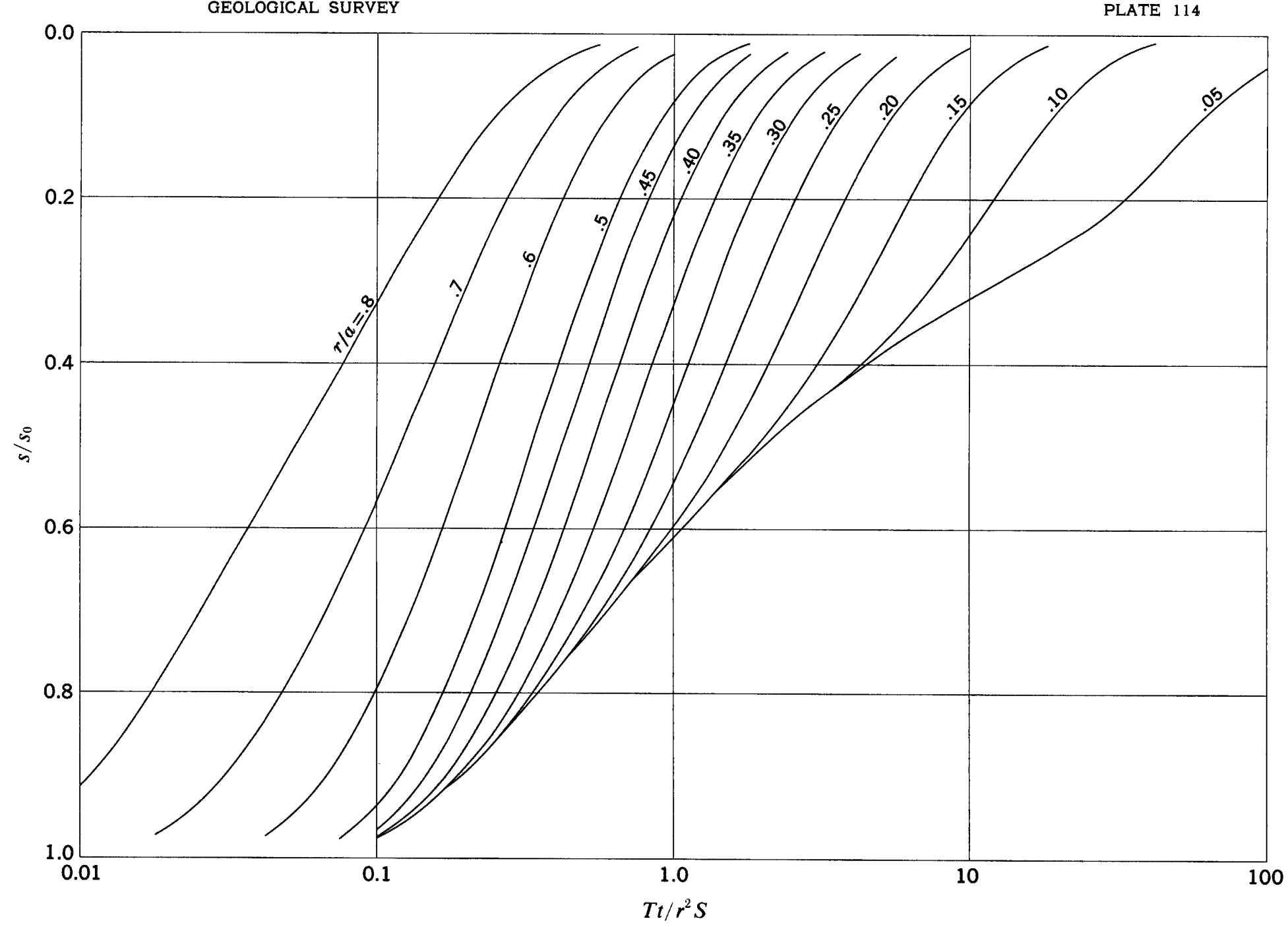

$s / s_{0}$ versus $T t / r^{2} S$ for $\theta_{0}=360^{\circ} ; \theta / \theta_{0}=0.20$ 


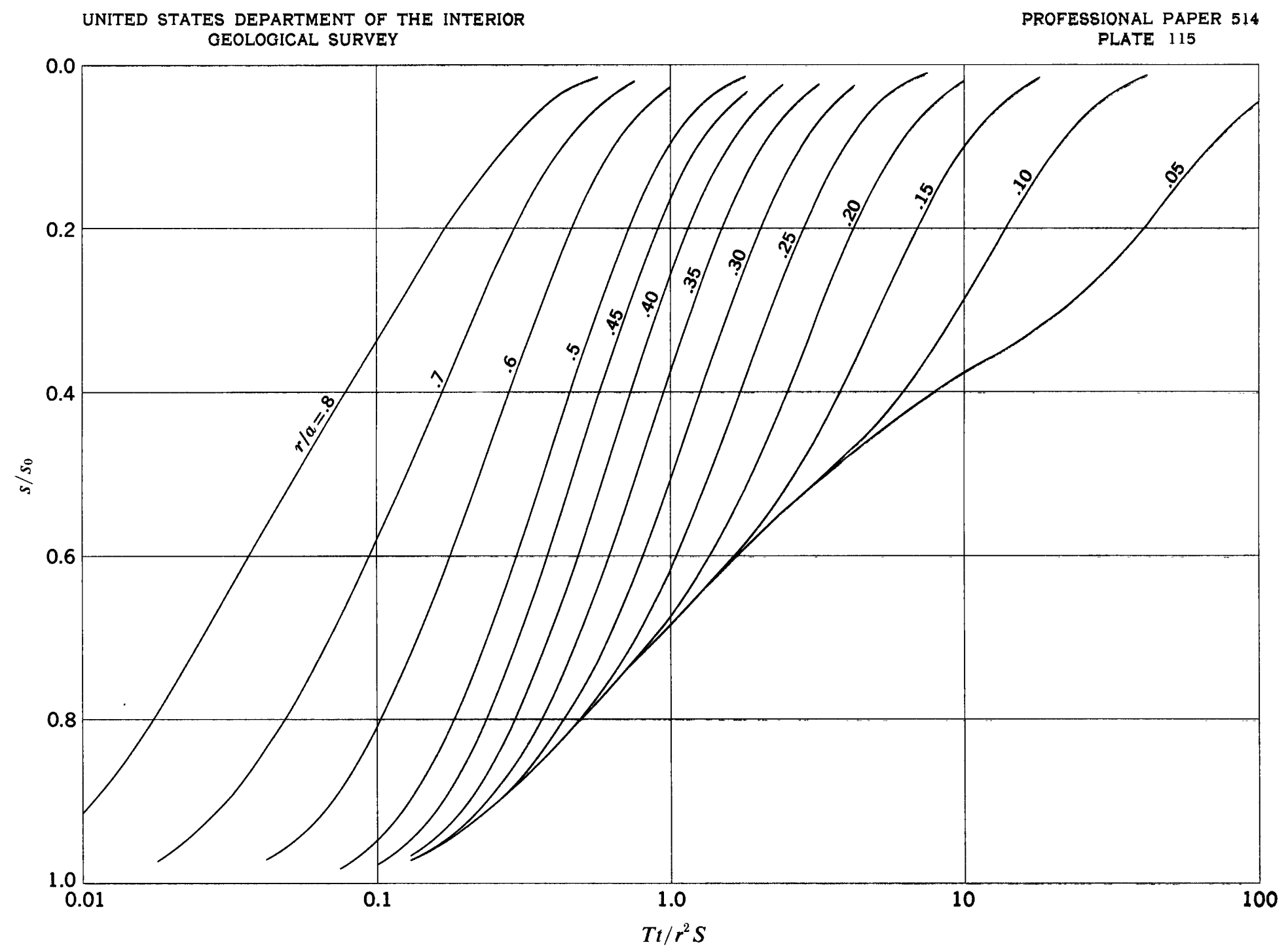

$s / s_{0}$ versus $T t / r^{2} S$ for $\theta_{0}=360^{\circ} ; \theta / \theta_{0}=0.25$ 

GEOLOOICAL SURVEY

PROFESSIONAL PAPER 514 PLATE 116

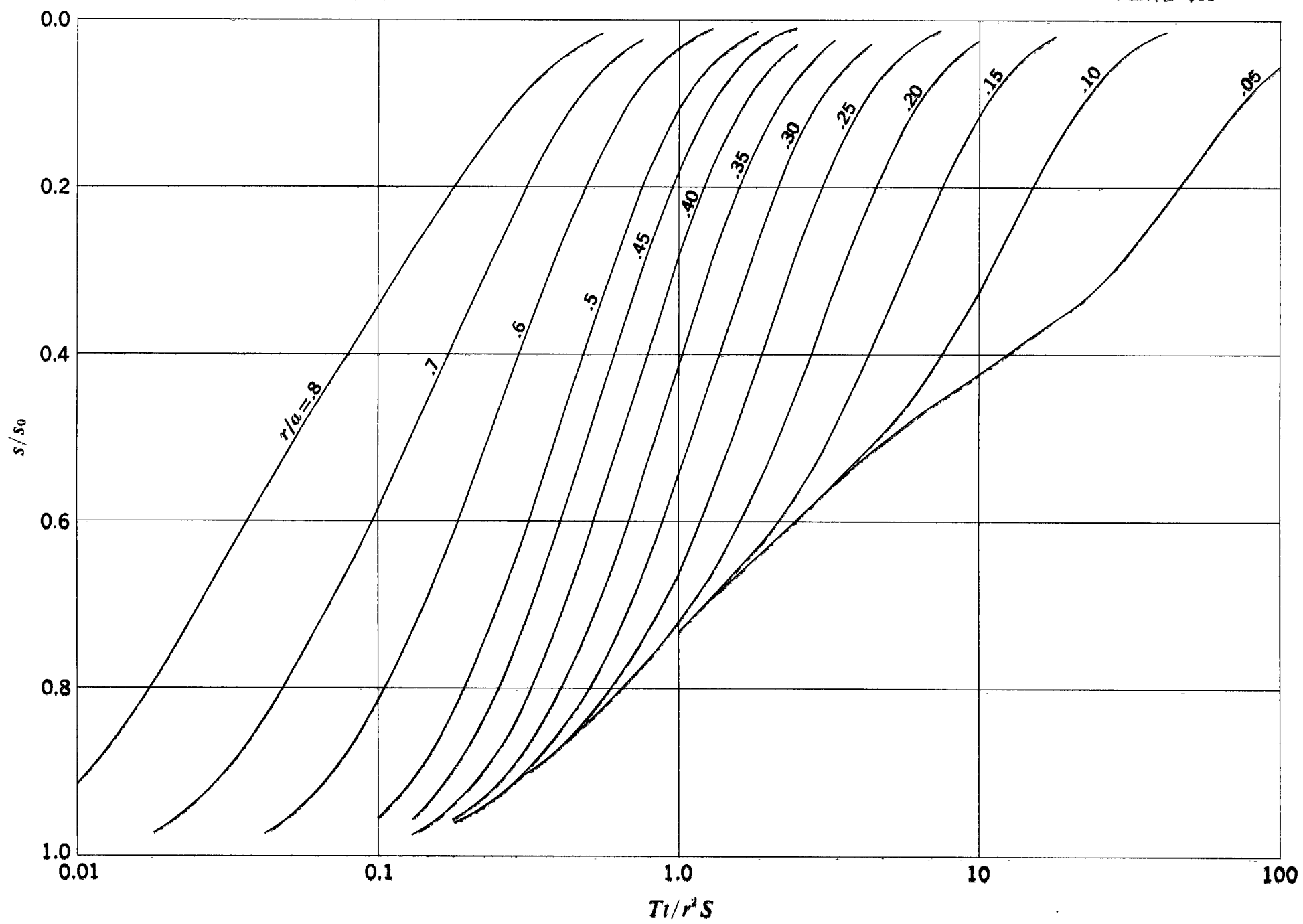

$s / s_{0}$ versus $T t / r^{2} S$ for $\theta_{0}=360^{\circ}: \theta / \theta_{0}=0.30$ 

GEOLOGICAL SURVEY PLATE 117

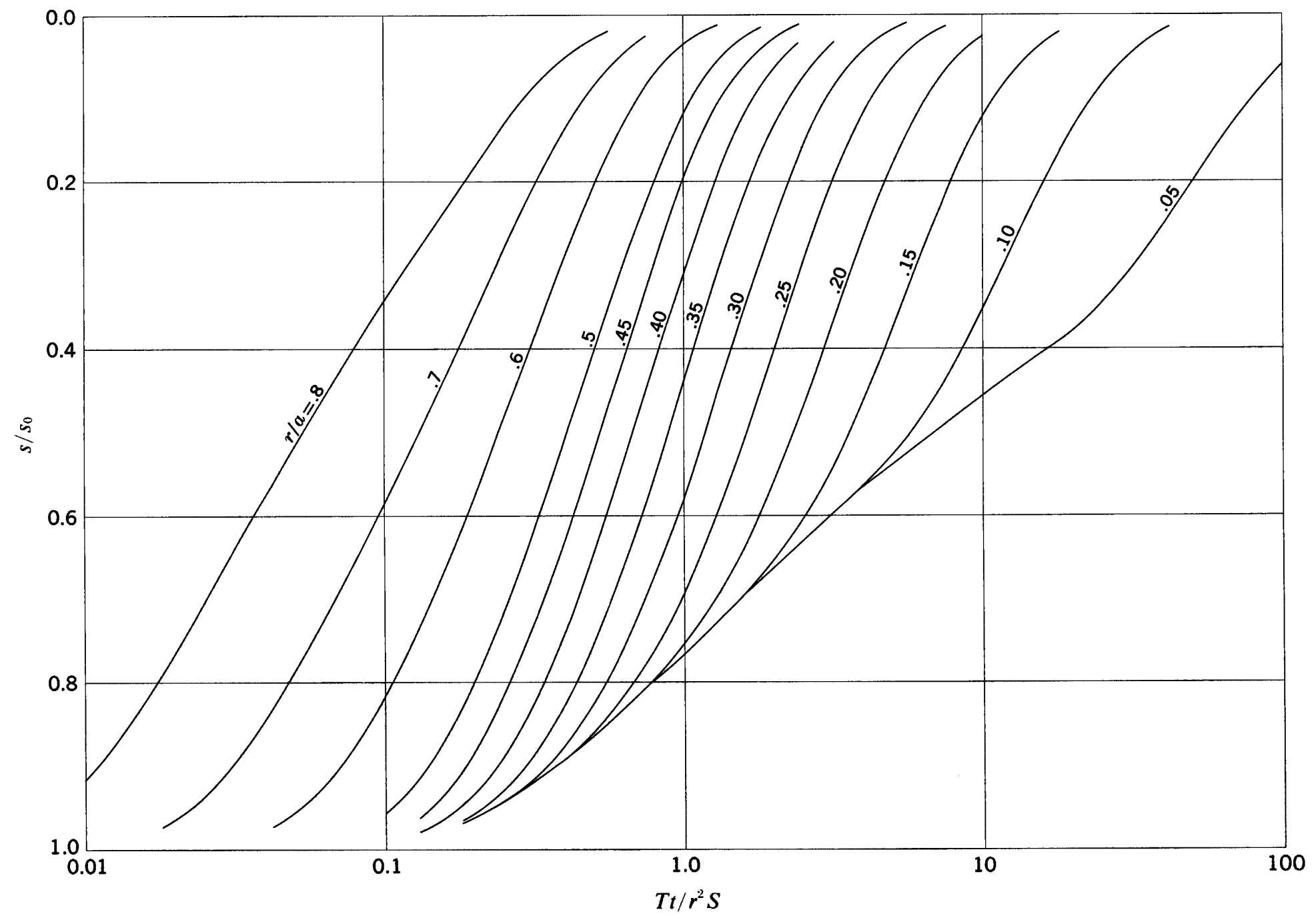

$s / s_{0}$ versus $T t / r^{2} S$ for $\theta_{0}=360^{\circ} ; \theta / \theta_{0}=0.35$ 
UNITED STATES DEPARTMENT OF THE INTERIOR GEOLOGICAL SURVEY
PROFESSIONAL PAPER 514 PLATE 118

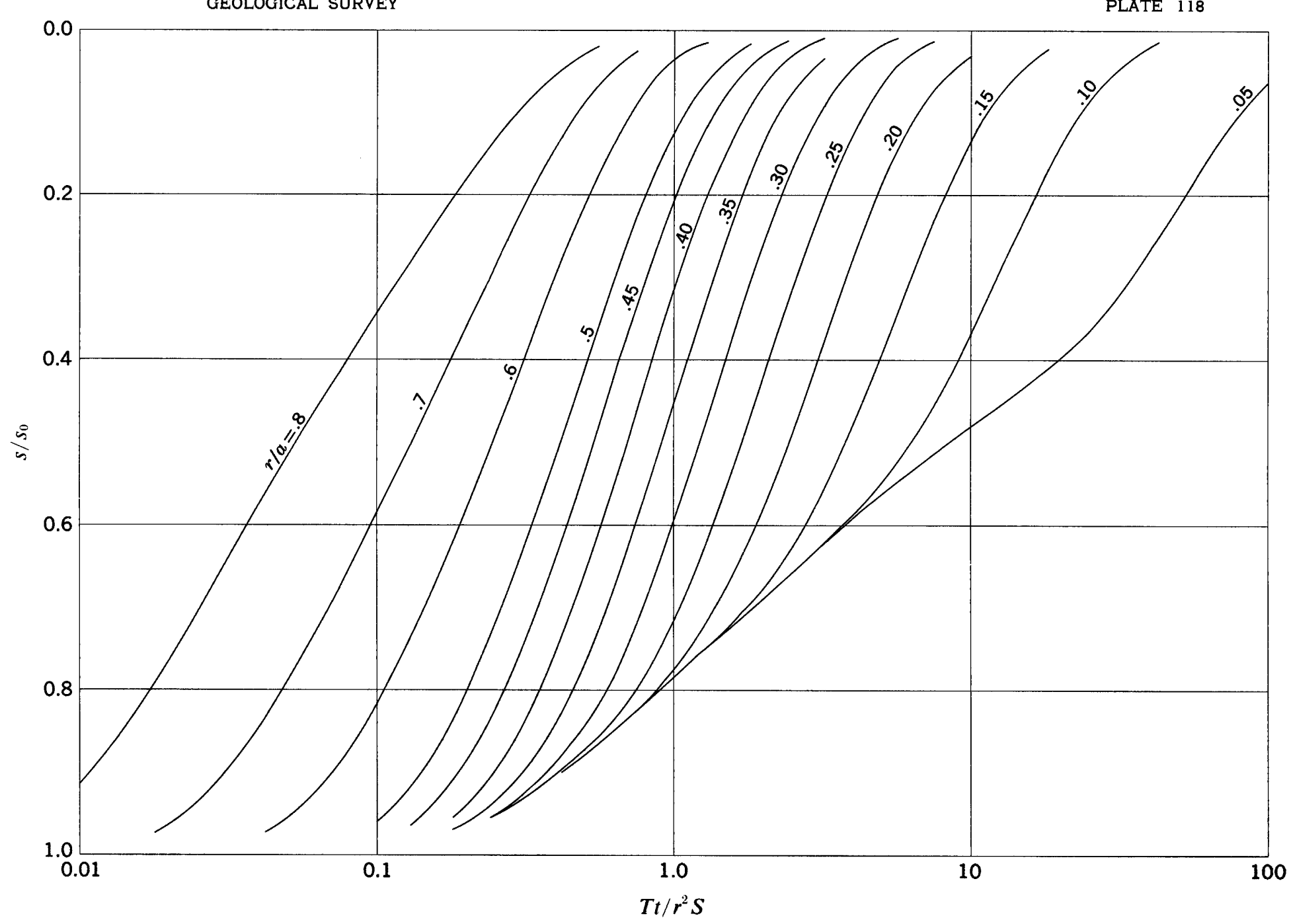

$s / s_{0}$ versus $T t / r^{2} S$ for $\theta_{0}=360^{\circ} ; \theta / \theta_{0}=0.40$ 
UNITED STATES DEPARTMENT OF THE INTERIOR GEOLOGICAL SURVEY
PROFESSIONAL PAPER 514 PLATE 119

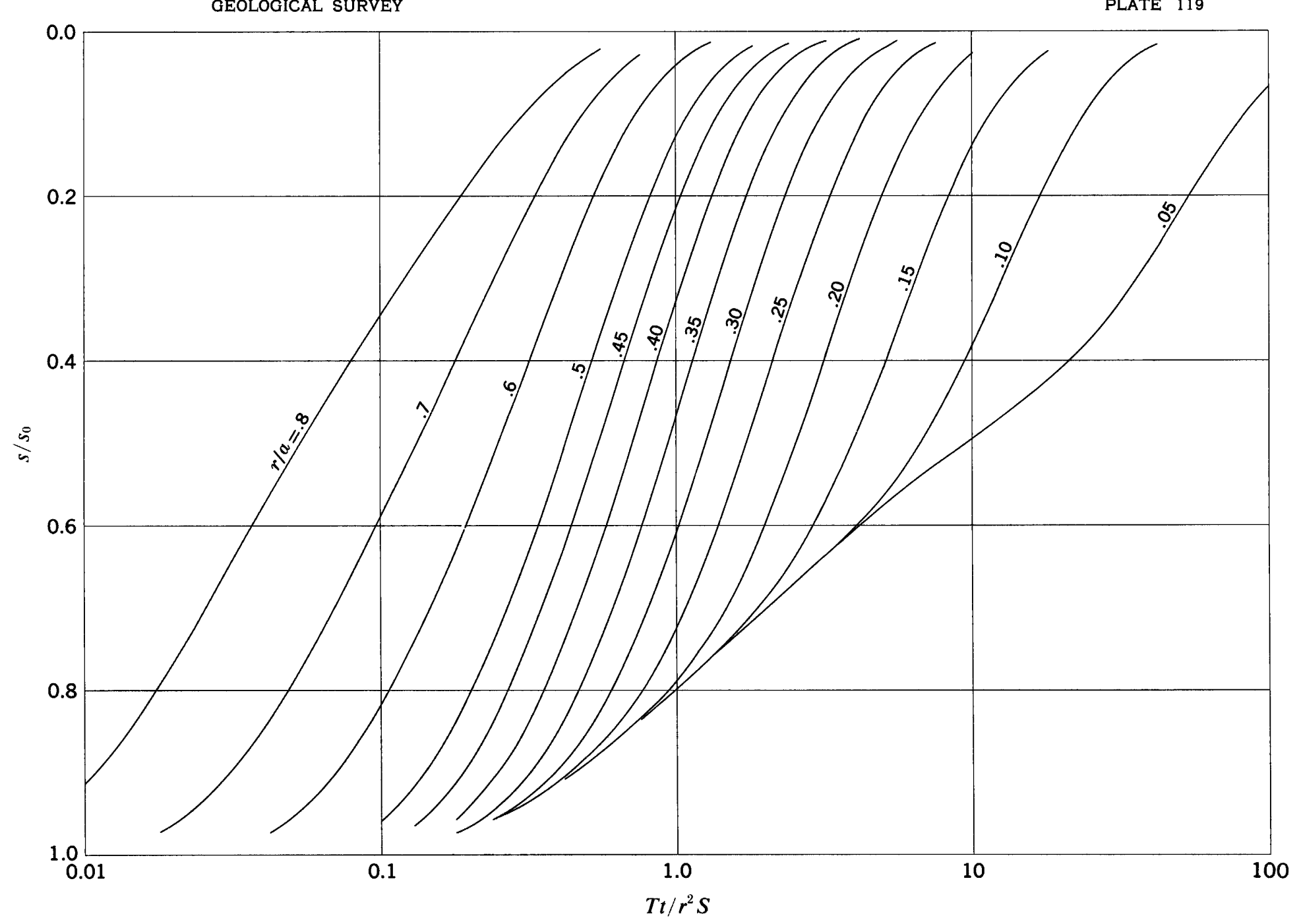

$s / s_{0}$ versus $T t / r^{2} S$ for $\theta_{0}=360^{\circ} ; \theta / \theta_{0}=0.45$ 


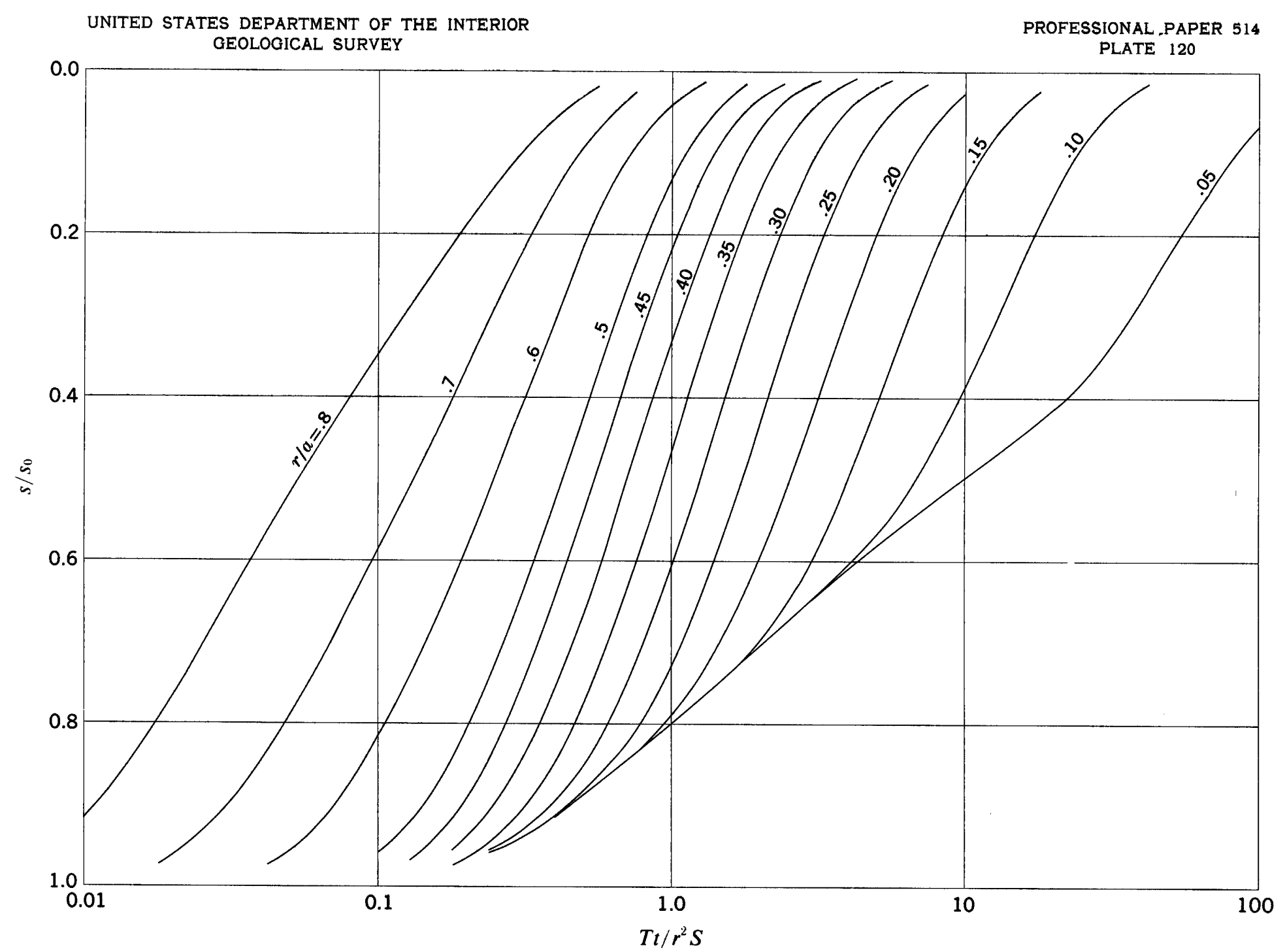

$s / s_{0}$ versus $T t / r^{2} S$ for $\theta_{0}=360^{\circ} ; \theta / \theta_{0}=0.50$ 